\title{
RAINBOW EXPERIENCES OF ACCESSING MENTAL HEALTH SUPPORT IN AOTEAROA NEW ZEALAND: A COMMUNITY-BASED MIXED METHODS STUDY
}

\author{
BY \\ GLORIA EILEEN FRASER
}

A thesis

submitted to Te Herenga Waka - Victoria University of Wellington in fulfilment of the requirements for the degree of Doctor of Philosophy

Te Herenga Waka - Victoria University of Wellington 2020 


\begin{abstract}
While we know that rainbow people in Aotearoa New Zealand (that is, people of diverse sexualities, genders, and sex characteristics) experience high rates of adverse mental health outcomes, we know much less about the extent to which Aotearoa's rainbow community members are receiving the mental health support they need. To address this gap I used mixed methods and a reflexive community-based approach to extend current understandings of rainbow mental health support experiences, and to explore how the provision of mental health care can be improved for rainbow people in New Zealand.
\end{abstract}

I first conducted interviews with 34 rainbow community young adults about their experiences of accessing mental health support. My thematic analysis showed that rainbow people across New Zealand faced significant structural barriers to accessing mental health support. Participants understood mental health settings as embedded within a heteronormative and cisnormative societal context, rather than as a safe place outside this context. This, together with a widespread silence from mental health professionals around rainbow identity, meant that participants actively negotiated coming out in mental health settings. Participants shared a variety of perspectives as to whether it should be standard practice for mental health professionals to ask about rainbow identities, but agreed on a number of subtle acts that could communicate a professional or service is rainbow-friendly. Knowledge about sexuality, gender, and sex characteristic diversity, together with clinical skills of empathy, validation, and affirmation, were described as key components for the provision of effective mental health support.

I conducted a second thematic analysis of data from a subset of the initial interviews, in which 13 participants discussed their experiences of accessing genderaffirming healthcare. Participants reported a lack of funding for gender-affirming healthcare in New Zealand, and described its provision a "postcode lottery"; the care available was largely dependent on the region participants were living in. Mental health assessments for accessing gender-affirming care were often described as tests of 
whether participants were "really" transgender, and participants discussed the need to express their gender in a particular way in order to access the healthcare they needed.

Thematic analyses of interview data informed the development of an online survey about rainbow peoples' experiences of accessing mental health support and gender-affirming healthcare in New Zealand $(n=1575)$. Survey results closely reflected interview findings, indicating that rainbow people have mixed experiences in New Zealand's mental health settings, and that accessing gender-affirming healthcare is a lengthy and convoluted process.

Finally, interview and survey data were used to develop a resource for mental health professionals, to guide their work with rainbow clients. I sought and incorporated feedback from key stakeholders $(n=108)$ during resource development. I then distributed the resource to mental health professionals around New Zealand, both in print and online.

Overall, my research shows that widespread knowledge gaps compromise the ability of New Zealand's mental health professionals to provide culturally competent support to rainbow clients. Knowledge from this thesis can be used to increase awareness of rainbow community members' mental health support needs, and to inform mental health professionals' training and self-reflection around sexuality, gender, and sex characteristic diversity. 


\section{Ngā mihi}

When I read a thesis I always jump first to the acknowledgements, and I have been eagerly waiting to finish this thing and write my own! I am so grateful to all the people that made this research happen. A PhD can be lonely at times so if any students are reading this, make sure you lean on those around you and take time out to do the things you love. Kia kaha, you will get there.

First, and most importantly, thank you to each and every person who met with me to share their experiences, completed an online survey, or gave feedback to improve the resource for mental health professionals. I really appreciate you being so generous with your time and for trusting me with your stories and your thoughts. Thank you also to everyone who helped spread the word about this research - I wouldn't have reached nearly as many people without your help.

I am incredibly grateful to all the community organisations, advocates and activists who gave guidance and support throughout this project. Thanks in particular to Tabby Besley, Ahi Wi-Hongi, and Toni Duder for the energy you gave this project, I admire you all so much for your passion and tireless work! Toni - thank you for the hours spent co-editing the resource over the phone and swapping cat photos, you were a joy to work with. Many more people supported this research than I can name here, but I would like to acknowledge Joey Macdonald, Taine Polkinghorne, Aych McArdle, Tommy Hamilton, Georgia Andrews, Jelly O’Shea, Alex Ker, Maggie Shippam, Alfie Van de Wiel, and the wonderful folk at UniQ Victoria. Thank you to Jordan Curtis and Bo Moore for your beautiful design and illustration work on this project, and for your unending patience with my (often nonsensical) requests!

Thank you to all those who funded this research - the Rule Foundation, the Oakley Mental Health Foundation, Victoria University of Wellington Postgraduate Students' Association, Te Rau Ora, Rainbow New Zealand Charitable Trust, and Rainbow Ride New Zealand. Thank you also to those who made it possible for me to complete eight (!) years of tertiary study - Victoria University of Wellington, the Sylff Association, Graduate Women New Zealand, Te Rūnanga o Ngāi Tahu, Te Rūnanga o Moeraki, the New Zealand Psychological Society, and the Ministry of Health. 
To my supervisors, Marc Wilson and Anita Brady, thank you for your wise advice and helpful feedback, for always checking in to see how I was going, for the laughter and tangential conversations, for coaching me to "think, thank, reject", and for shouting me many a hot drink. It was such a relief to know I could take all my quandaries to supervision and be taken absolutely seriously. I have learned a great deal about integrity, humility, and respect from you both, and I hope we have the opportunity to keep working together in future.

Thank you to my Youth Wellbeing Study whānau, the best lab in all the world! Our meetings always made me feel connected and optimistic for the week ahead. Thank you for eating my baking (even when it didn't quite go to plan) and for humouring me with success circles and animal of the week. Special thanks to my office mates, Catherine Pihema and Kylie Sutcliffe, and to Jess Garisch for being the warmest and most encouraging researcher and clinician I have ever met. I would also like to acknowledge those in the wider YWS community who supported this project, particularly Mike Cole for your encouragement and thoughtful resource feedback.

In my time at Victoria University I have been lucky to befriend the loveliest people the School of Psychology has to offer. Thank you Emma Tennent, Fiona Grattan, Sophie Hedley, Jess Shaw, Jess La, and Sam Duffy for your company, great conversation, and for fiercely standing up for all you believe in. Thank you to the writing groups I have been a part of, especially the various iterations of the Māori and Pacific Students Rōpu and Pomodoro Fridays, and to my Clinical classmates for your support along the way.

Thank you to those faraway friends who listened, gave pep talks, and read drafts particularly Kate Young, Lucy Cowie, Franziska Pöschl, and Ximena Smith - and to Eva Penny, Vincent Rhodes, Joshua Power, and my other Roskill pals for making sure I managed to squeeze some parties in too.

Kealagh Robinson and Kate McLeod - my dearest frolleagues - you belong in more than one of the above paragraphs, but deserve your very own! I am endlessly grateful for the hundreds of hours you've spent celebrating my successes, listening to my woes, giving feedback on my work, and being a sounding board for all decisions. Thank you for each and every cup of tea, gin and tonic, gif, meme, and tweet. Doing 
this $\mathrm{PhD}$ has brought me lots of amazing opportunities, but making lifelong friends in you both was definitely the most special part.

Thank you to my biggest cheerleaders, my parents Donna and Shane Fraser, for your endless love and support. The longer I spend working in mental health the more I understand the effort you put into giving me a safe and secure base from which to explore the world. I admire you both so much as people and hope I can always be there for you as you have been for me. Thank you also to my Grandma Claire, Stepmum Jo, and in-laws Anne and Peter for backing me all the way, and to my sisters Cordelia and Frida for being absolute rays of sunshine.

Thank you to Sooty for all the snugs in years leading up to this thesis, and to Poppy for all the company during. Your purrs, chirps and insistence on falling asleep on my laptop keyboard have never failed to melt my heart. You are the Very Best Cats.

Finally, to Mark Keith Pearce, who started this thesis as my partner and finished as my husband! Thank you for all you've done and continue to do - reminding me to come home from work, batting my phone away when I check emails on the weekend, cooking for me, making sure I don't take myself too seriously, giving big hugs when I shed the odd tear, taking me on pub crawls and listening to my virtually nonstop chatter... You make everything easier and much more fun. I can't wait to see where we end up next.

Aroha nui ki a koutou - now you can call me Dr Fraser! 


\section{Table of Contents}

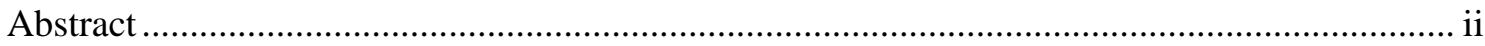

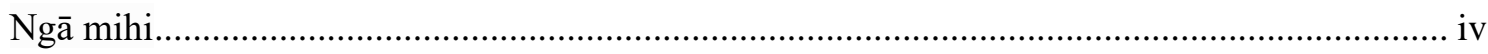

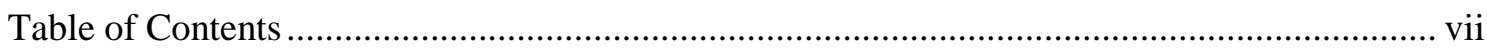

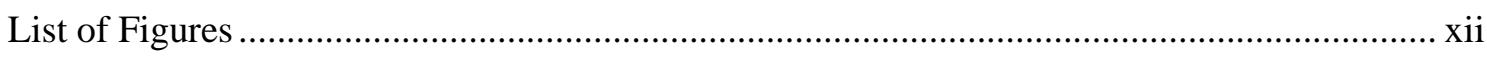

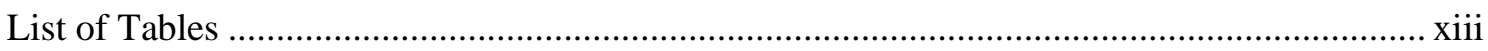

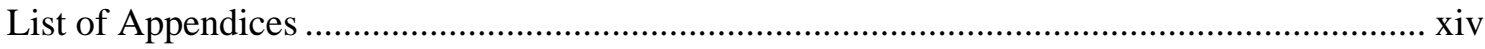

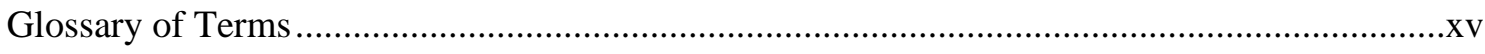

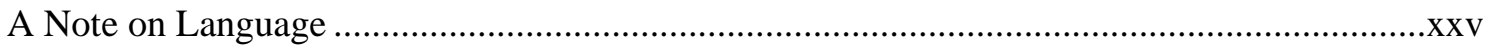

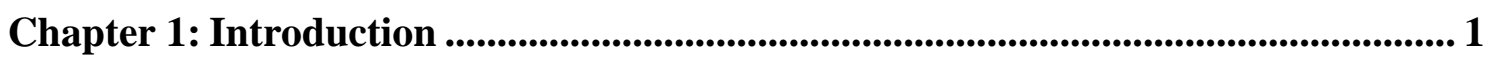

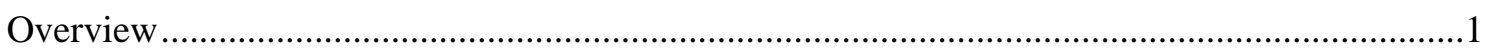

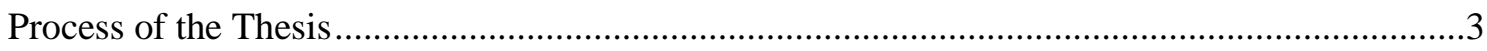

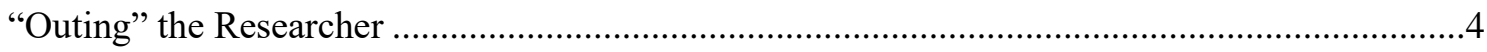

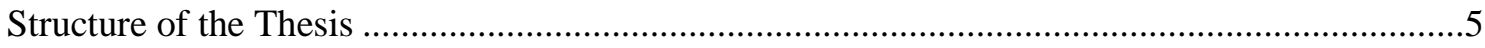

Chapter 2: Setting the Scene ....................................................................................................... 8

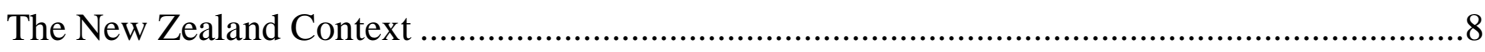

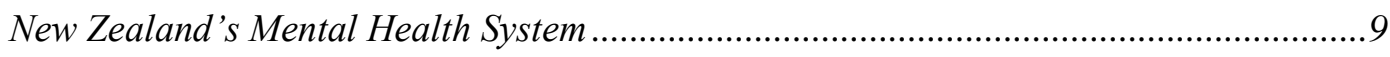

Rainbow Rights in New Zealand................................................................................

Mental Health and Wellbeing in Rainbow Communities ........................................................14

Mental Health and Wellbeing in New Zealand's Rainbow Communities.........................16

Historical Understandings of Rainbow Mental Health ..........................................................17

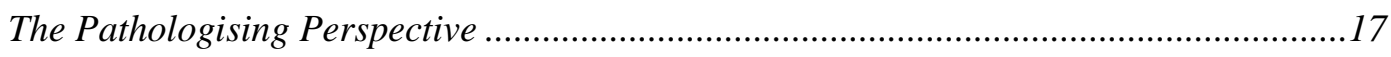

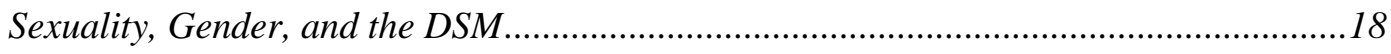

Contemporary Understandings of Rainbow Mental Health ......................................................21

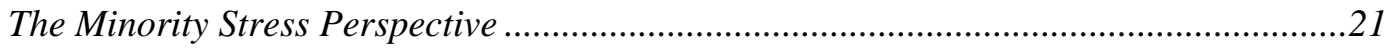

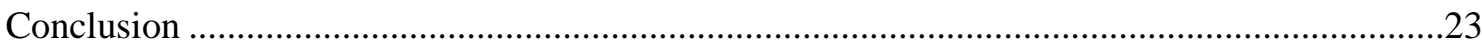


Rainbow Experiences of Accessing Mental Health Support...................................................... 24

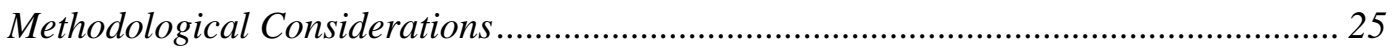

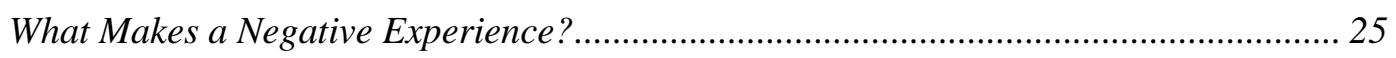

What Makes a Positive Experience? ................................................................................. 30

The Impact of Negative Therapeutic Experiences......................................................... 31

Experiences of Groups Within the Rainbow Community ................................................. 32

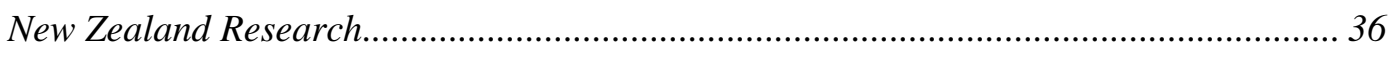

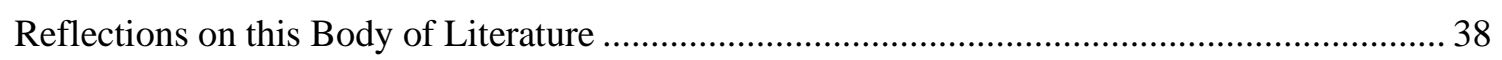

Has the Nature of Negative Experiences Changed Over Time? ....................................... 39

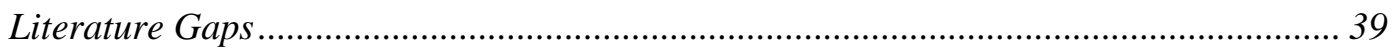

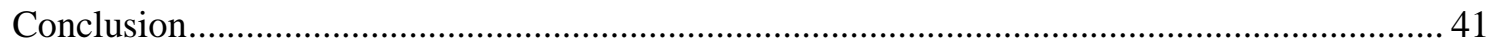

Chapter 4: Positioning and Theoretical Framework ........................................................43

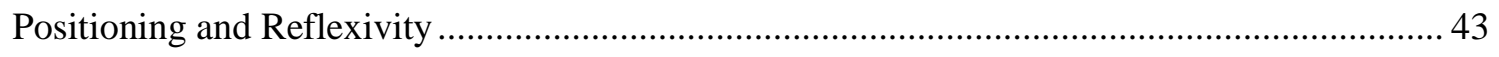

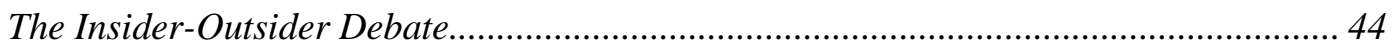

“Working the Hyphen” and Occupying "the Space Between ”........................................ 47

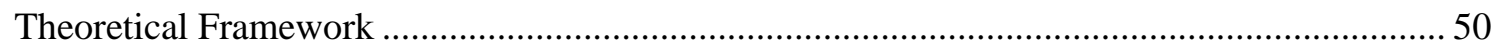

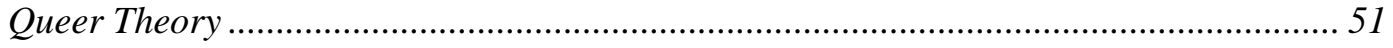

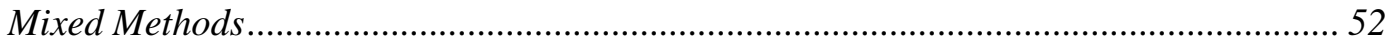

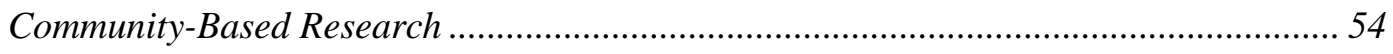

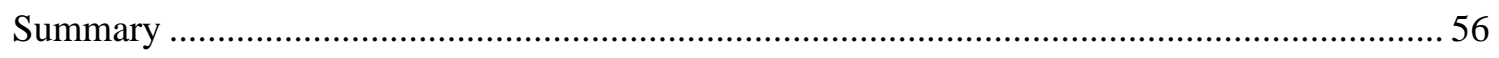

Chapter 5: Interview Methodology ....................................................................................57

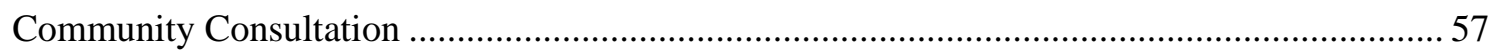

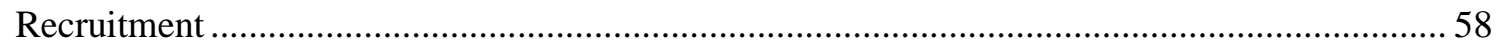

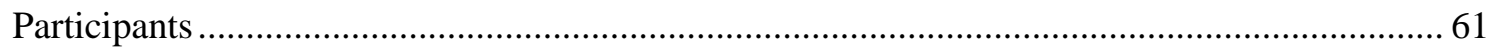

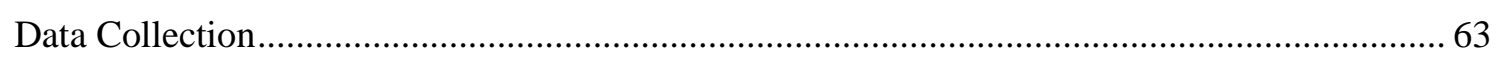

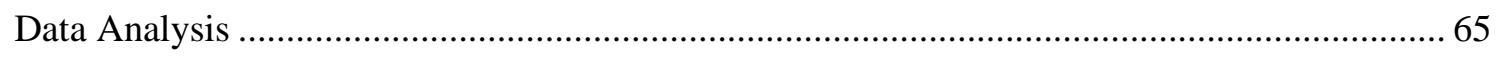


Thematic Analysis

Theme One: "Accessing Healthcare is like a Game of Strategy".. .72

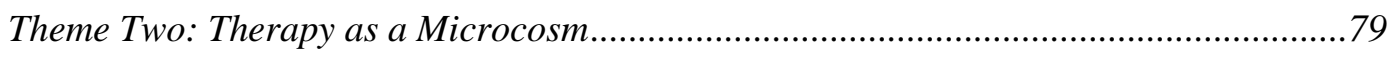

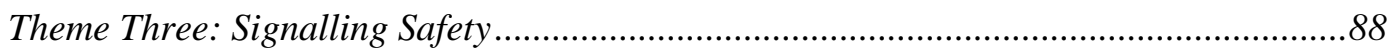

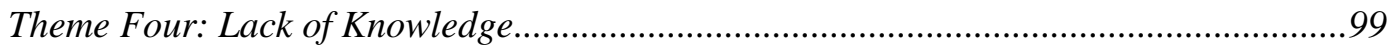

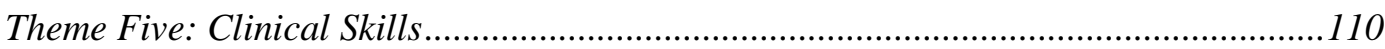

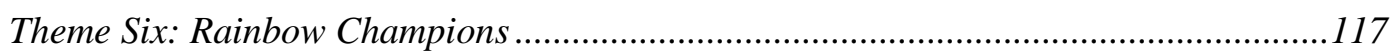

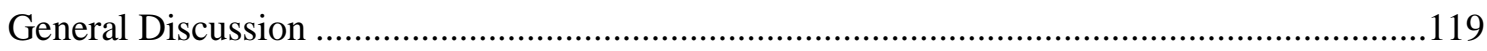

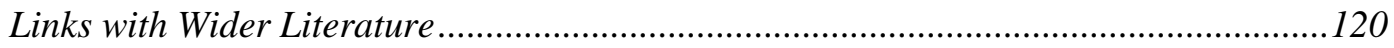

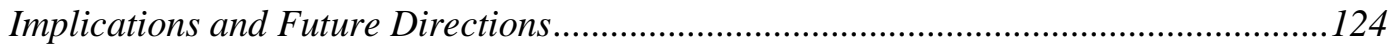

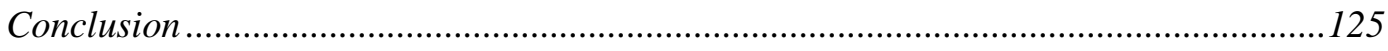

Chapter 7: Analysis of Interviews About Accessing Gender-Affirming Care ...... 127

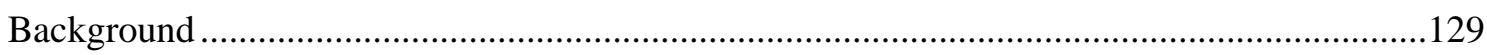

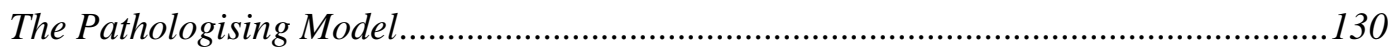

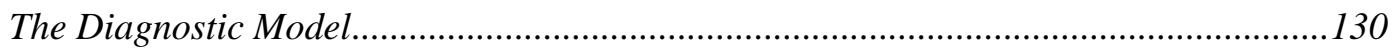

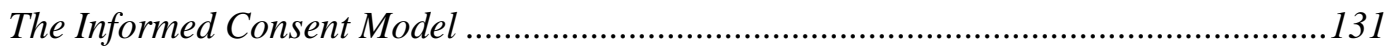

Standard Practice in New Zealand ..........................................................................131

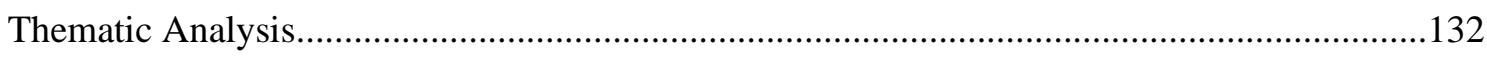

Theme One: Lack of Funding and the "Postcode Lottery”...........................................132

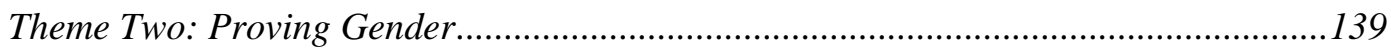

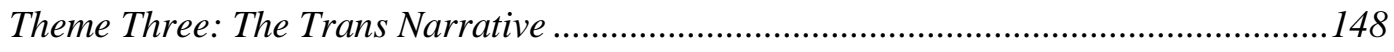

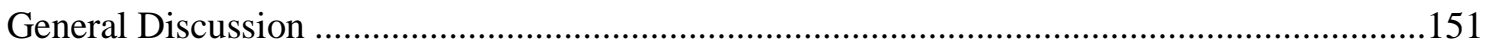

Participants ' Experiences in Context ……............................................................ 152

Implications, Reflections, and Future Directions .......................................................153

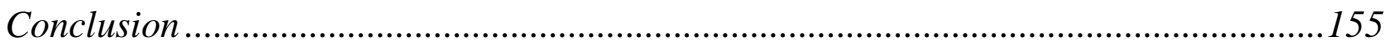


Chapter 8: Online Survey

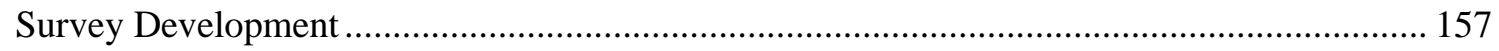

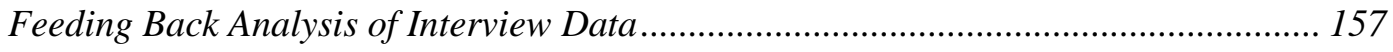

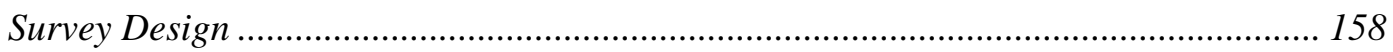

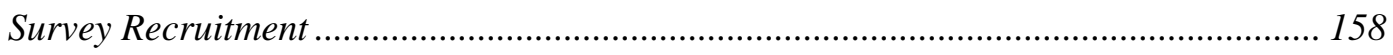

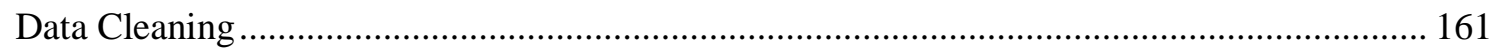

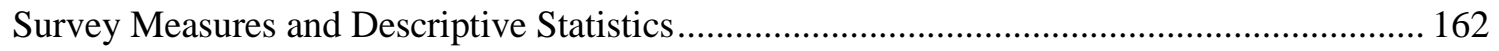

Demographic Information and Wellbeing ……......................................................... 162

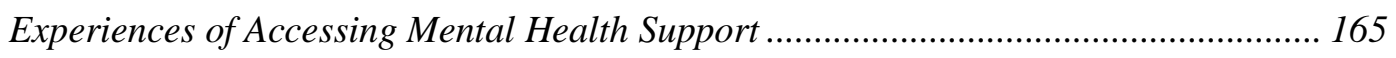

Experiences of Accessing Gender-Affirming Healthcare.............................................. 175

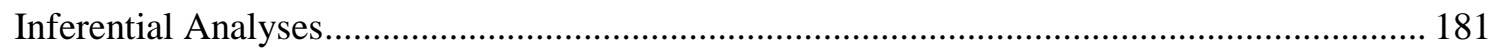

Demographic Differences on Select Survey Items ..................................................... 181

Past Experiences, Worries, and Comfort ............................................................... 183

Past Experiences and Overall Helpfulness .............................................................. 186

Discrimination Experiences and Future Worries ...................................................... 189

Has the Experience Improved over Time? .................................................................. 189

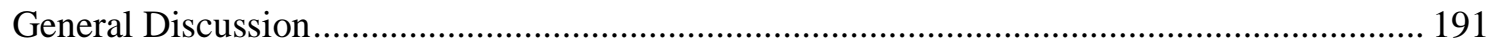

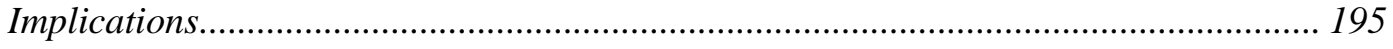

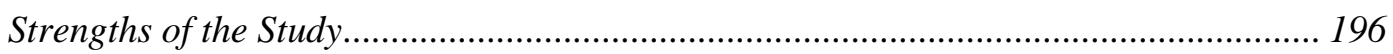

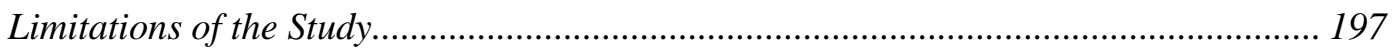

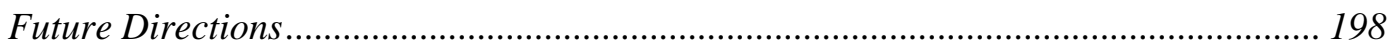

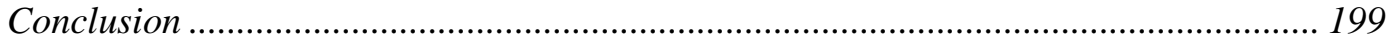

Chapter 9: The Resource ....................................................................................201

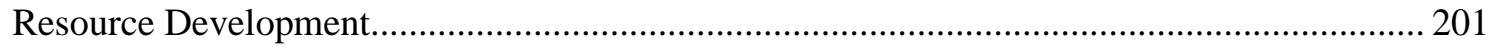

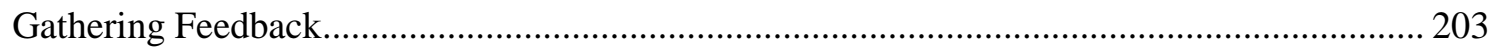

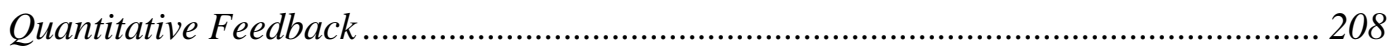

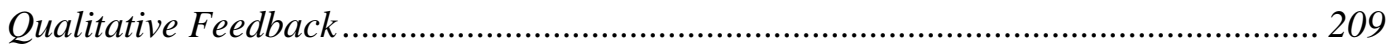


Incorporating Feedback

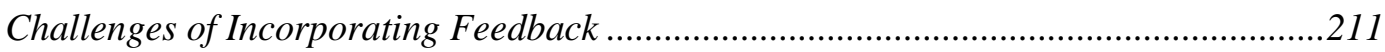

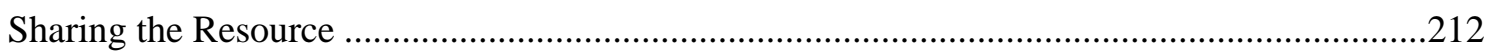

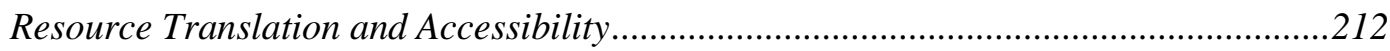

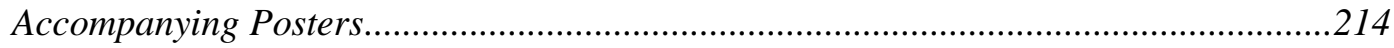

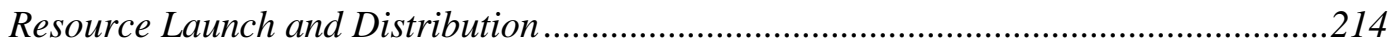

The Resource

Chapter 10: Reflections and Final Thoughts ..................................................................... 246

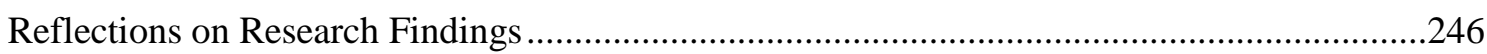

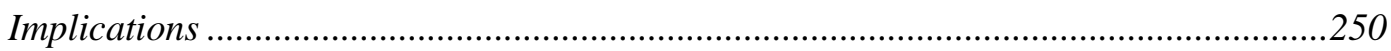

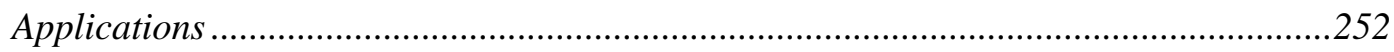

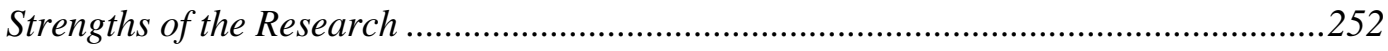

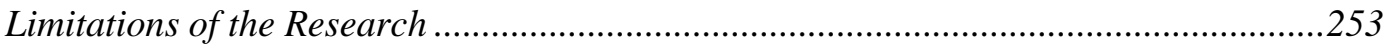

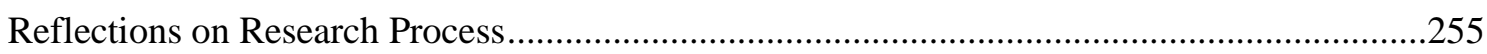

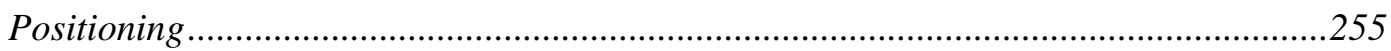

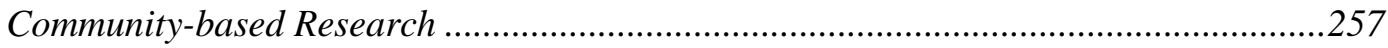

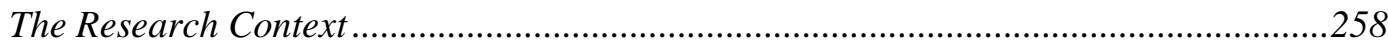

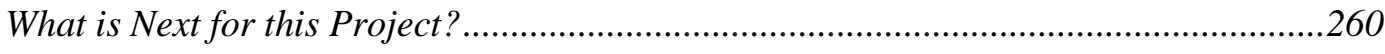

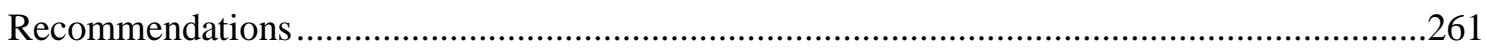

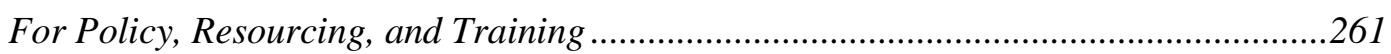

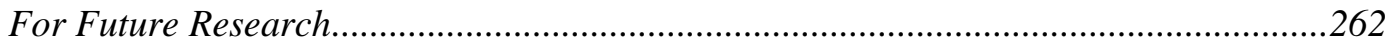

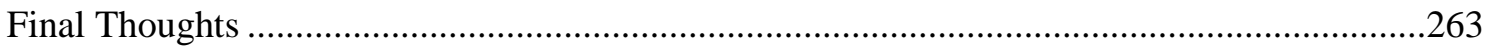

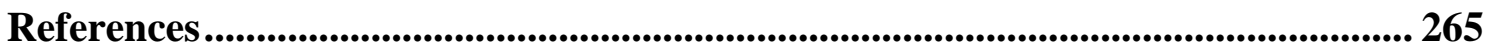




\section{List of Figures}

Figure 1. Graphical depiction of Lucksted's continuum of negative experiences.... 26

Figure 2. Theoretical framework for the current study.........................................5 50

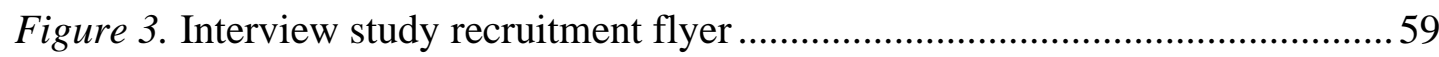

Figure 4. Final thematic map for analysis of interviews about accessing mental health

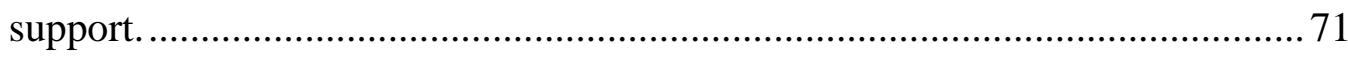

Figure 5. Final thematic map for analysis of interviews about accessing gender-

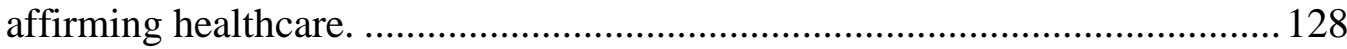

Figure 6. Online survey recruitment flyer ......................................................... 160

Figure 7. Tweet of Poppy (my beautiful tuxedo cat) promoting the online survey. 161

Figure 8. Participants' sexual orientation. ........................................................... 163

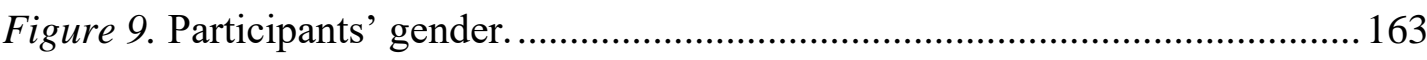

Figure 10. Comfort when meeting new mental health professionals. .................... 168

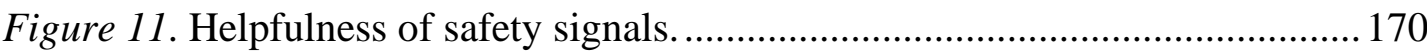

Figure 12. Positive experiences when accessing mental health support.................. 171

Figure 13. Negative experiences when accessing mental health support. ............... 172

Figure 14. Worries in mental health settings. ..................................................... 174

Figure 15. Importance of identity in mental health settings. ................................. 175

Figure 16. Experiences during assessments to access gender-affirming healthcare.178

Figure 17. Respect, knowledge, and the trans narrative in assessments for gender-

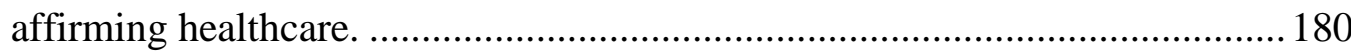

Figure 18. Resource feedback recruitment flyer for mental health professionals. .. 204

Figure 19. Resource feedback questions for mental health professionals. .............. 206

Figure 20. Resource feedback questions for rainbow participants. ....................... 207

Figure 21. Poster encouraging sharing pronouns.................................................. 244

Figure 22. Poster encouraging sharing feedback ................................................ 245

Figure 23. Poster of top tips for mental health professionals. ................................245

Figure 24. Poster for creating a welcoming space ............................................... 245 


\section{List of Tables}

Table 1. Participants' intersecting identities 165

Table 2. Access to gender-affirming healthcare services 176

Table 3. Bivariate correlations between negative experiences and comfort/worries 184

Table 4. Bivariate correlations between positive experiences and comfort/worries 185 Table 5. Multiple regression of overall helpfulness based on positive experiences 187 Table 6. Multiple regression of overall helpfulness based on negative experiences 188 Table 7. Breakdown of resource feedback 208

Table 8. Resource feedback from mental health professionals and rainbow participants 209 


\section{List of Appendices}

*All appendices can be found online at https://tinyurl.com/GFraser-PhD-Appendices

Appendix A: Interview study ethical approval

Appendix B: Interview study ethical considerations

Appendix C: Interview study information sheet

Appendix D: Interview study guide

Appendix E: Interview study consent form

Appendix F: Online survey ethical approval

Appendix G: Online survey ethical considerations

Appendix H: Online survey measures

Appendix I: Online survey detailed demographic information

Appendix J: Table showing crosstabs between trans identification and gender-affirming healthcare access

Appendix K: Tables showing mean differences based on demographic information

Appendix L: Resource feedback ethical approval

Appendix M: Resource feedback email to mental health professionals

Appendix N: Resource feedback information sheet for mental health professionals

Appendix O: Resource feedback email to rainbow participants

Appendix P: Resource feedback information sheet for rainbow participants 


\section{Glossary of Terms}

I have emboldened each of these terms the first time it appears in my thesis to indicate it is defined in this glossary. Here, te reo Māori words are italicised to differentiate them from te reo Pākehā words (English words), however Māori words are not italicised throughout the thesis. Most definitions related to sexuality, gender, and sex characteristic diversity are adapted from the Gender Minorities Aotearoa (2017) glossary, and definitions of te reo Māori words are adapted from maoridictionary.com. Other sources are cited throughout the glossary.

Age out/Aging out

Agender

A person who does not identify with or conform to any gender.
When a young person leaves a formal system of care designed to provide services below a certain age level, as they are no longer eligible for those services.

Allyship

When someone outside of the rainbow community supports rainbow people, promotes equality, stands up to discriminatory behaviour, and works to understand the complexities of the rainbow community.

Aotearoa

Asexual
The Māori name for New Zealand. Its most popular translation is "land of the long white cloud".
A person who is not primarily motivated by sexual drive and sexual attractions, though they may experience sexual attraction or 
have sexual relationships for reasons other

than primary sexual attraction.

\section{Bigender}

A person who experiences two gender identities, either simultaneously or varying between the two. These could be male and female but could also include nonbinary gender identities.

\section{Bisexual}

Traditionally referred to a person who is attracted to both men and women. Currently being redefined by some bisexual rights activists to mean that one is attracted to both their own gender, as well as other genders.

Call out/calling out

To hold someone accountable for their words or actions.

\section{Cisgender (cis)}

A prefix or adjective that means "not trans": cis people identify with the gender assigned to them at birth.

\section{Civil union}

A legally recognized relationship, similar to marriage.

\section{Come out/coming out}

The process by which one accepts and/or comes to identify one's own sexuality or gender identity, as well as by which one shares one's sexuality or gender identity with others (to "come out" to friends, etc.). 


\section{Crowdfunding}

Deadname
"Allows founders of for-profit, artistic, and cultural ventures to fund their efforts by drawing on relatively small contributions from a relatively large number of individuals using the internet, without standard financial intermediaries" (Mollick, 2014, p. 1).

A birth name, or name used prior to transition, that someone no longer uses.

Clinical term referring to dissonance between one's assigned sex and/or one's body, and one's personal sense of self.

Gender-affirming healthcare Can refer to any health service that affirms and validates a person's gender, including counselling and support with social transition. In this thesis I use this term predominantly to refer to medical transitionrelated services, including hormone therapy, surgeries (mastectomy, hysterectomy, orchidectomy, and breast augmentation), and ancillary services (facial hair removal and voice training).

\section{Genderfluid}

A gender which varies over time. A genderfluid person may at any time identify as male, female, nonbinary, or some combination of identities. 
Genderqueer

Grey literature

Heteronormativity and cisnormativity

An umbrella term with a similar meaning to nonbinary. It can be used to describe any gender identities other than man or woman.

Information that is not published or is published outside of traditional academic publishing or commercial publishing channels.

Pervasive societal assumptions that everyone is (or should be) straight and cis (Bauer et al., 2009; Schilt \& Westbrook, 2009).

Together, these societal structures operate by rendering queer and trans identities invisible, as well as by trivialising, repressing, or stigmatising queer and trans identities. Expressions of heteronormativity and cisnormativity may be more difficult to identify than overt discrimination but can be experienced as equally harmful by rainbow community members (Sue, 2010).

\section{Heterosexism and cissexism Ideological systems that deny and stigmatise behaviours, identities, relationships, or communities that deviate from heterosexual and/or cis norms (Herek, 1990; Serano, 2007).}

\section{Homophobia and transphobia}

Umbrella terms for a range of negative attitudes (e.g. fear, anger, intolerance, or discomfort) towards gay and trans people. 'Homophobia' does not explicitly include xviii 
queer people who are not gay but is often used to refer to the whole queer community.

Hui Gathering, meeting, assembly, seminar, or conference.

Hyperromantic

A person who feels extremely strong romantic feelings and may value a romantic bond over anything else.

\section{Intersectionality}

The systematic study of the interrelationships of various forms of marginalisation (e.g. that based on race, gender, sexuality, class, religion, ability, and other sociopolitical and cultural categories; see Crenshaw, 1989, 1991). Intersectionality emphasises that identity is always made up of multiple facets, and that the nature of marginalisation changes when these facets intersect (McCall, 2005).

\section{Intersex/variations in sex} characteristics
Terms used when a person's sex characteristics (e.g. sexual anatomy, reproductive organs, hormonal patterns and/or chromosomal patterns) are more diverse than the typical definitions of male and female. their actions. 
Koha

Gift, present, offering, donation,

contribution.

Lesbian

Women who have the capacity to be

attracted romantically, physically, and/or

sexually to some other women.

LGBT/LGBTQIA+

Lesbian, gay, bisexual, trans, often extended

to include queer (or questioning), intersex,

asexual, plus others who are not straight

and/or cis.

Mahi

Most commonly used to mean "work".

Matauranga Māori

Māori epistemologies or ways of knowing.

\section{Microaggressions}

"Brief and commonplace daily verbal, behavioral, or environmental indignities, whether intentional or unintentional, that communicate hostile, derogatory, or negative slights and insults toward members of oppressed groups" (Nadal, 2008, p. 23).

\section{MVPFAFF}

A Pasifika umbrella term for rainbow identities coined by Phylesha Brown-Acton:

Mahu, Vakasalewa, Palopa, Fa'afafine, Akava'ine, Fakaleiti (leiti), Fakafifine (PrideNZ, 2011).

Acknowledgements. 
The principal Māori iwi (extended kinship group) of the southern region of New Zealand.

Nonbinary

Othering
Any gender identity which does not fit the gender binary. The gender binary is a system of viewing gender as consisting solely of two categories, termed male and female, in which no other possibilities for gender or anatomy are believed to exist.

"A process that identifies those that are thought to be different from oneself or the mainstream, and ... can reinforce and reproduce positions of domination and subordination" (Johnson et al., 2004, p. 253).

\section{Pākeh̄̄}

New Zealander of European descent. Often used to refer to any non-Māori New Zealander.

\section{Pansexual} gender, explicitly includes trans and nonbinary genders.

\section{Passing}

In the context of gender, refers to a person's ability to be read as the gender one wishes to be read as (usually used in a binary cis context). The term 'passing' is falling out of favour as it is seen to imply that one should desire to look cis. 
Performative allyship/ally theatre/performative wokeness
A phenomenon in which "an individual from a majority or privileged group (white/straight/cis/abled) professes their support of and/or solidarity with a marginalized group (identifies themselves as an ally) in a way that either isn't helpful to that group, draws attention away from that group, or actively harms that group" (Mae, n.d., para. 3).
Polyamory/non-monogamy

Queer
The practice of, or desire for, intimate relationships with more than one partner, with the knowledge of all partners.

Broadly used to indicate that one rejects heteronormativity and is not heterosexual though sometimes queer is also used by heterosexual trans people.

An umbrella term for a range of negative attitudes (e.g. fear, anger, intolerance, or discomfort) that one may have towards members of the queer community.

\section{Questioning}

The process of considering or exploring one's sexual orientation and/or gender identity.

\section{Straight/heterosexual} emotionally, physically, and/or sexually attracted to women, and women who are 
primarily emotionally, physically, and/or sexually attracted to men.

Takatāpui

A term that historically meant "intimate companion of the same sex" (Williams, 1871, p.147), but has been reclaimed to refer to all non-heterosexual, non-cis, and/or intersex Māori people (similar to rainbow; Kerekere, 2017a).

Top surgery Surgical procedures to either remove breast tissue (mastectomy, also called masculinising chest surgery), or to increase breast size (breast augmentation or chest construction, also called feminising breast surgery).

\section{Toxic masculinity}

"The constellation of socially regressive male traits that serve to foster domination, the devaluation of women, homophobia, and wanton violence" (Kupers, 2005, p. 714).

\section{Transgender (trans)}

An umbrella term for people whose gender identity and/or gender expression differs from what is culturally typically associated with the gender/sex they were assigned at birth. People under the trans umbrella may describe themselves using one or more of a wide variety of terms or may simply use trans. Some people who fit this definition may not consider themselves to be under the trans umbrella or trans. 
Transition

Whakapapa

Whānau

xxiv

XXiv
Moving from being seen as one's birth assigned gender to one's actual gender.

Transition generally initially includes social elements (e.g. changing one's clothes, hair, name, changing the gender marker on one's legal documents, binding breasts or wearing breast forms), and may also include medical treatments such laser hair removal, hormone therapy, or various surgeries.

Genealogy, lineage, or descent.

Family, extended family. 


\section{A Note on Language}

Many umbrella terms are used to describe the diverse group of people who are not straight, not cisgender (cis), and/or who are intersex: LGBT or LGBTQIA+; rainbow; minority sexualities, genders, and sex characteristics; takatāpui; queer, transgender (trans), and intersex; sexuality, gender, and sex characteristic diverse. Throughout this thesis I alternate between use of the umbrella terms "rainbow", "queer, trans, and intersex", and "sexuality, gender, and sex characteristic diverse". I use these terms for their inclusivity, and because these are the terms used most commonly used by participants and community partners. I acknowledge that the umbrella terms I use do not work for everyone. ${ }^{1}$ I also use "rainbow community/ies" at points in this thesis, while recognising that not all rainbow people seek community, have access to community or feel welcome in rainbow communities. Where cited material uses other identity terms, I follow their usage. At all times, I use participants' own identity terms.

Throughout this thesis I use Māori language and concepts. Māori are the indigenous people of New Zealand, and mātauranga Māori diverges considerably from Western frameworks (Royal, 2012). I draw on Māori language and knowledge in an effort to normalise te reo Māori (one of New Zealand's official languages), because of my own identity as Māori, and because te reo Māori expresses concepts and experiences with no direct English translation; use of te reo Māori is essential for understanding peoples' perspectives and experiences in this place.

"Mental health support" is a broad term that can encompass individual, group and family-based therapy, crisis services, peer support, therapeutic communities, psychosocial programmes and groups, and inpatient treatment. For the purpose of this thesis I use the terms "mental health support" and "therapy" interchangeably to refer to face-to-face discussions about mental health with a mental health professional, such as a psychologist, counsellor, psychiatrist, therapist, social worker, or mental health nurse. Support received from general practitioners (who are often the gateway to other

\footnotetext{
${ }^{1}$ Space constraints prevent me from elaborating further on the reasons for and against the use of different terms, but see Ferris (2006), Jagose (1996), Kulick (2000), and Leap and Boellstorf (2004) for discussion.
} 
services) is outside the scope of this thesis, however the insights gained in this thesis will likely inform the work of general practitioners and other primary health providers.

Finally, with regard to language - the words we use to talk about rainbow peoples' lives and experiences really matter; they have the potential to welcome and include, and to affirm and validate identities, but they can also be used to exclude, to denigrate, and to disempower. Language use is made even more complicated by the fact that terminology in this area can shift and change quickly. I have no doubt that the language used in this thesis will be considered outdated soon after it is published. As such, this thesis reflects a particular cultural moment. 


\section{Chapter 1: Introduction}

\section{Overview}

The fields of mental health care and mental health research have long and fraught relationships with sexuality, gender, and sex characteristic diversity, characterised by the pathologisation of rainbow people (Clarke, Ellis, Peel, \& Riggs, 2010). While the last half century has seen positive shifts - in particular, the removal of homosexuality from the Diagnostic and Statistical Manual of Mental Disorders (DSM) - the American Psychiatric Association (APA) receives ongoing criticism for the retention of gender dysphoria as a diagnosis in the DSM-5 (Lev, 2016), and the needs of rainbow people are rarely considered in mainstream psychological research (Diamond, 2003).

Research within LGBTQ psychology, which examines rainbow mental health and wellbeing, demonstrates that queer, trans, and intersex people around the world experience high rates of adverse mental health outcomes as a result of stress caused by stigma, violence, and discrimination (Budge, Adelson, \& Howard, 2013; FredriksenGoldsen et al., 2013; King et al., 2008; Lucassen, Stasiak, Samra, Frampton, \& Merry, 2017). Research from Aotearoa - a country with a reputation for being at the forefront of social change ${ }^{2}$ - suggests that mental health disparities within rainbow communities reflect those seen internationally (Clark et al., 2014; Fergusson, Horwood, \& Beautrais, 1999; Fergusson, Horwood, Ridder, \& Beautrais, 2005; Pitts, Couch, Mulcare, Croy, \& Mitchell, 2009).

Policy and community-based initiatives that combat sexuality and gender-based stigma and discrimination are key in addressing rainbow mental health disparities (Fredriksen-Goldsen et al., 2014). There is an increasing awareness, however, that interventions must be employed at individual, institutional, and societal levels in order

\footnotetext{
${ }^{2}$ See Chapter 2 for an overview of the New Zealand context, as well as examples of social change regarding sexuality, gender, and sex characteristic diversity.
} 
to improve the health and wellbeing of queer, trans, and intersex people (Hatzenbuehler, 2010). At the individual level, intervention involves the provision of high-quality mental health support to rainbow community members. But are rainbow people receiving the mental health support they need? To date, very little research has explored the experiences of rainbow community members who have accessed some form of mental health support in Aotearoa. This thesis aims to fill this gap.

There is a growing international literature examining the experiences of rainbow people who access mental health support (Bell \& Weinberg, 1978; Filice \& Meyer, 2018; King et al., 2003; Liddle, 2000; White \& Fontenot, 2019). Findings vary, but indicate that, overall, instances of overt discrimination within mental health settings have decreased over time (King, Semlyen, Killaspy, Nazareth, \& Osborn, 2007). There is, however, evidence to suggest that poor service provision has lingered in other forms. Recent studies report that mental health professionals rarely challenge heteronormativity and cisnormativity in their practice, commonly lack knowledge about rainbow needs and experiences, and reproduce a cultural silence around sexuality, gender, and sex characteristic diversity (e.g. Evans \& Barker, 2010; McCann \& Sharek, 2014; McIntyre, Daley, Rutherford, \& Ross, 2011; Shelton \& Delgado-Romero, 2011).

It is unclear whether the experiences of rainbow people who access mental health support in New Zealand mirror those reported in the wider literature, as only a handful of studies on this topic have been reported in our context. These have either been limited to specific groups within the rainbow community (e.g. to men who have sex with men, or to lesbians; Semp, 2006; Welch, Collings, \& Howden-Chapman, 2000), have combined results with those from other countries (Couch et al., 2007), or are methodologically flawed, limiting the extent to which findings can inform practice recommendations (Birkenhead \& Rands, 2012). Moreover, no study to date has examined the experiences of trans people who present to New Zealand's mental health services to access gender-affirming healthcare. Given the increasing number of people accessing gender affirming healthcare in New Zealand (Delahunt, Denison, Sim, Bullock, \& Krebs, 2018), such research is urgently needed. International research may not shed light on the experiences of rainbow people in Aotearoa due to our unique indigenous, social, and economic context (see Chapter 2 for a description of this context). 
The first aim of my programme of research is to gain an in-depth understanding of the experiences of rainbow people who have accessed mental health support in New Zealand. The second aim is to consider how these findings can be used to improve the provision of mental health support for New Zealand's rainbow people. My research questions are:

- What are the experiences of rainbow people who access mental health support in New Zealand?

- What are the experiences of trans people who undergo mental health assessments for gender-affirming healthcare in New Zealand?

- How can these findings inform the provision of high quality and responsive mental health care for queer, trans, and intersex people in New Zealand?

\section{Process of the Thesis}

At the start of my thesis journey I came up with several possible options for answering my research questions. Initially, I planned to conduct interviews with rainbow people who had accessed mental health support, rainbow activists and advocates, and mental health professionals, to ensure my findings were informed by a range of perspectives. Other ideas for later included an online survey of some or all of these groups, a content analysis of resources for mental health professionals about supporting rainbow clients, and the development of my own resource for mental health professionals.

After interviewing 34 rainbow people who had accessed mental health support, it became clear that increased rainbow cultural competence among New Zealand's mental health professionals was urgently needed to improve the quality of mental health care for queer, trans, and intersex people. I suspected that if I were to interview mental health professionals and rainbow activists and advocates, they would tell me the same thing. As such, I decided against conducting further interviews, and instead prioritised developing a resource for mental health professionals, to guide their work with rainbow clients. I first conducted an online survey, as I felt that my resource should be informed by the stories of a wider group of rainbow New Zealanders; it was possible that the experiences of my 34 interview participants were not reflective of common rainbow therapy experiences. 
Thus, my final project had three stages: (1) interviews with rainbow people who had accessed mental health support, (2) online surveys with a wider group of rainbow people who had accessed mental health support, and (3) the development of a resource for mental health professionals, to guide their work with rainbow clients.

\section{"Outing" the Researcher}

When Finlay (2002) talks of "outing" the researcher, she refers to the process of engaging in self-aware analysis of a researcher's perspective and presence. In conducting this research, however, I have had to come out quite literally - as straight and cis - for the first time in my life. Many of the participants in my interview study examined my information sheet with great interest before our interview, posing questions such as, "So... if you're straight, then why are you doing this?" (on the sheet I stated that "I identify as a straight, cis woman of Pākehā and Ngāi Tahu descent"). Over the course of my research, rainbow community members, acquaintances, my whānau, and more than one Uber driver asked questions along the same lines. What were my motivations for taking on this project? Why was I dedicating three years of my life to something I had no lived experience of? Did I have the right to do this research? Was I sure I'm not really queer?

For a long time I struggled to provide answers to these questions, because I felt I could give a dozen different reasons for my choice of topic. In isolation, none seemed to explain why this research is so important to me. On reflection, however, I have realised that a range of experiences throughout my life led to this thesis - conversations with friends who (often nervously) shared they were queer or trans, being elected as the chairperson of my university feminist collective (and questioned as to whether my feminism was truly intersectional) and learning about social constructionism for the first time in a class on the psychology of gender. By the time I finished my undergraduate degree I was fascinated by the seemingly arbitrary systems of power and privilege (particularly in relation to sexuality and gender) that determine much of our day-to-day lives. Around this time, I was also volunteering in mental health organisations, and had discovered a passion for psychological research.

Eventually, I found ways to bring my interest in gender and sexuality together with my interest in psychology and mental health. I conducted research about self-injury 
among young people of different sexual orientations (Fraser et al., 2018), and the ongoing and problematic use of a male/female tick box to measure gender in psychological research (Fraser, 2018; Fraser, Bulbulia, Greaves, Wilson, \& Sibley, 2019). I began to feel concerned about the level of rainbow cultural competence within my own field - in the first few months of my clinical psychology programme one lecturer used the word 'transsexual' in class, another denied that 'they' was a valid personal pronoun, and I heard classmates speculate as to whether a client's sexual orientation could have resulted from a history of abuse. ${ }^{3}$ I discovered that in my three years of postgraduate study, I would receive a single two-hour guest lecture about working with gender diverse and intersex clients. I had long heard from queer and trans friends that they had struggled to find mental health professionals who they found helpful, and I wondered whether New Zealand's mental health professionals were equipped to support their rainbow clients. After a search of academic literature on rainbow experiences in mental health settings yielded barely any New Zealand studies, ${ }^{4}$ my $\mathrm{PhD}$ was born.

I reflect on my positioning throughout this thesis because my various identities and subject positions have shaped my decision-making at every stage of the research process. As I discuss in Chapter 4, I see my research questions, methodology, data analysis, and interpretation as inextricably linked to the person that I am. In saying this, I make efforts to avoid crossing into autobiographical territory throughout this thesis as Denzin (2006) points out, self-reflexivity is distinct from self-obsession!

\section{Structure of the Thesis}

The next chapter provides context for the current research, with a short overview of New Zealand's mental health system and the history of rainbow rights in New Zealand. I then briefly review literature on the health and wellbeing of rainbow

\footnotetext{
${ }^{3}$ To be clear, these examples reflect a lack of knowledge around rainbow terminology and experiences; the word transsexual has largely fallen out of use (although some still use this term to self-identify), 'they' is indeed a valid personal pronoun, and the search for a cause of a client's sexual orientation fails to acknowledge that diverse sexualities are part of usual human experience - we rarely search for the cause of something unless we want to change it.

${ }^{4}$ This absence was particularly concerning in light of the historical pathologisation of sexuality, gender, and sex characteristic diversity in mental health settings, and given that societal stigma and discrimination has such a profound impact on rainbow peoples' mental health. I elaborate on these ideas in Chapter 2.
} 
communities, both in New Zealand and internationally. Finally, I explore how understandings of rainbow mental health disparities have evolved over the last century.

Chapter 3 reviews literature about rainbow experiences of accessing mental health support. I examine common elements that define negative and positive experiences and consider how the nature of negative experiences have changed over time. I then describe experiences specific to groups within the rainbow community, review New Zealand research on this topic, and conclude by outlining the ways in which my research will address gaps in this body of literature.

In Chapter 4, I reflect on my positionality within this research. I first review literature on insider/outsider positioning in qualitative research and discuss ways in which 'outsider' researchers can conduct research that benefits the communities they work with. I then outline my theoretical framework, including brief overviews of queer theory, community-based research, and mixed methods.

Chapter 5 describes my interview methodology. I begin by outlining the process of building relationships with rainbow community organisations and advocates. I then describe the process of participant recruitment, data collection, and data analysis.

I present my first thematic analysis of interviews with a diverse group of rainbow people in Chapter 6. Here I outline the six key themes I identified in relation to participants' accounts of accessing mental health support in Aotearoa. Chapter 7 details my second thematic analysis of interview data. In this chapter I present three themes I identified in relation to trans participants' experiences of accessing gender-affirming healthcare. In Chapter 8, I document the process of using interview findings to create an online survey exploring the experiences of a wider group of rainbow people who have accessed mental health support in Aotearoa. Here I discuss survey design and survey distribution and present an overview of key findings. I conclude each of Chapters 6, 7, and 8 with a general discussion my findings, exploring their significance and wider implications.

Chapter 9 describes the development of a resource to guide mental health professionals in their work with rainbow clients based on interview and survey data. I outline the process of drafting the resource, seeking feedback from rainbow community members and mental health professionals, and incorporating feedback into the final 
resource. I then present the resource that was printed and distributed to mental health professionals around New Zealand.

In Chapter 10 I reflect on my overall research findings, situate them within the wider research literature, and explore their theoretical and practical significance. I also outline the strengths and limitations of my research and reflect on what it was like to carry out community-based research in New Zealand from my perspective, and at this time. I end with recommendations for future research and policy. 


\section{Chapter 2: Setting the Scene}

Here, I set the scene for my research with an overview of the mental health system in New Zealand, as well as the history of rainbow rights in New Zealand. New Zealand's unique history of colonisation, current political and social climate, and public health service provision informs the experiences of rainbow people in mental health settings; this background is essential for putting my later research findings and resource development in context. I then briefly outline the sizeable international body of research examining the health and wellbeing of rainbow community members and the smaller body of research about the health and wellbeing of New Zealand's rainbow people. Finally, I outline the ways in which understandings of queer, trans, and intersex identities and mental health have evolved over the last century.

\section{The New Zealand Context}

New Zealand is often described as one of the most socially and politically progressive countries in the world. As well as being the first self-governing nation in which women could vote, New Zealand was the first country to have all of its top constitutional positions simultaneously held by women, ${ }^{5}$ and is well known for implementing a nuclear-free policy in the mid 1980s (Grimshaw, 2013; Wilson, 2013). In 2017, New Zealand was ranked ninth overall in the world on the Social Progress Index, an aggregate index of social and environmental indicators capturing three dimensions of social progress: Basic Human Needs, Foundations of Wellbeing, and Opportunity (Porter, Stern, \& Green, 2017).

Despite its rosy reputation Aotearoa faces significant challenges and is arguably less progressive than recent media reports suggest (e.g. Badham, 2018; Graham-McLay, 2018). New Zealand has the highest youth suicide rate in the developed world (UNICEF, 2017), concerningly high levels of family violence (New Zealand Family Violence Clearinghouse, 2017), and a history of restrictive and punitive abortion law,

\footnotetext{
${ }^{5}$ Queen, Governor-General, Prime Minister, Chief Justice, and Parliament's Speaker.
} 
with abortion criminalised up until March 2020 (Ministry of Health 2020). As I will outline in this section, New Zealand's record of public healthcare provision, rainbow human rights, and rainbow health and wellbeing follow this general trend of forwardmovement in some respects, and a worrying lack of progress in others.

\section{New Zealand's Mental Health System}

In the early $20^{\text {th }}$ century New Zealand set out to create a fully funded national health service, with the Social Security Act (1938). The act was never implemented in full, due to widespread opposition from private sector medical professionals (Easton, 2002). Instead, New Zealand developed a dual health system in which mental health services (as well as maternity and hospital services) were government-funded, while general practitioners were permitted to charge a fee over and above government subsidies for consultations (Ashton, 2005). As such, in Aotearoa, free or subsidised mental health support is available through the Ministry of Health, educational institutions, and non-governmental community organisations.

Over the last half century, mental health services in New Zealand have shifted from an institutional model of care (based on compulsory inpatient treatment) to a recovery model of care (primarily based on voluntary treatment in community settings; Ministry of Health, 2015). Following the closure of all psychiatric hospitals in the early 1990s and the establishment of the Mental Health Commission ${ }^{6}$ in 1996, New Zealand's mental health sector focussed on developing community-based support services, integrating Māori models of health in service delivery, and launching a national antidiscrimination campaign combatting mental health stigma (Brunton, 2011; Vaughan \& Hansen, 2004). New Zealand subsequently gained an international reputation for innovation in mental health service delivery (O’Hagan, Reynolds, \& Smith, 2012).

The election of a conservative National Party government in 2008 "took mental health off the health priority list," and led to substantial cuts to mental health funding (O’Hagan et al., 2012, p. 59). Between 2008 and 2017 demand for public mental health

\footnotetext{
${ }^{6}$ The Mental Health Commission was an autonomous Crown entity with a systemic monitoring role. The Commission was established following a 1988 government enquiry, the Mason Report, into the adequacy of New Zealand's mental health services (Brunton, 2011).
} 
services increased by $60 \%$, while funding increased by less than $30 \%$ (Wiggins, 2017). The Mental Health Commission was prematurely disestablished in 2012, and nongovernmental organisations suffered significant reductions in funding (New Zealand Association of Psychotherapists, 2016). From 2016 onwards, New Zealand's mental health system received considerable media attention for its state of 'crisis', with service users reporting lengthy waiting times and ever-increasing severity thresholds to meet eligibility for publicly funded treatment (see, for example, Campbell, 2017; McAllen, 2016; Wright, 2016).

In 2017, the centre-left New Zealand Labour Party formed a coalition government with the Green Party (a left-wing, environmentalist party) and populist New Zealand First. Shortly thereafter, the Government announced an Inquiry into Mental Health and Addiction to develop recommendations for a better mental health and addiction system for Aotearoa (Ardern, 2018). The Inquiry panel received more than 5,200 submissions and attended over 400 meetings around the country before releasing a report that described a mental health system failing to meet the needs of many New Zealanders (Government Inquiry into Mental Health and Addiction, 2018). Rainbow communities were cited as one of several minority groups with unmet mental health needs and especially poor experiences within New Zealand's mental health services.

In response to the Inquiry report the Government promised $\$ 455.1$ million to fund a new universal frontline mental health service, aiming to help 325,000 people access mental health services (Bennett, 2019). Questions remain, however, about who will staff this service, given the "chronic skills shortage in the sector" (Deguara, 2019, para. 7). Rainbow communities also received a funding boost, with the establishment of a Rainbow Legacy Fund that will administer $\$ 1$ million in grants to support organisations that improve mental health and wellbeing outcomes in New Zealand rainbow communities (Ardern \& Robertson, 2019). While these announcements brought new hope for improving access to mental health support in New Zealand, at the time of writing public mental health services continued to face significant challenges, with District Health Board (DHB) psychologists engaging in strike action due to low pay, understaffing, and a lack of professional support (Martin, 2019). 


\section{Rainbow Rights in New Zealand}

\section{Legality of sexual activity and discrimination protections}

In 1840, with the signing of the Treaty of Waitangi, ${ }^{7}$ New Zealand adopted British law, criminalising anal sex between men (Rishworth, 2007). This law was expanded in 1893 to include any sexual activity between men; penalties included hard labour, flogging, and life imprisonment (Dorey, 2006). Sex between women has never been criminalised in New Zealand, though Laurie (2003) points out that this does not mean lesbian relationships were accepted. Women in "abnormal sexual relationships" (p. 84) could be sent to government institutions or committed for psychiatric treatment.

The 1970s saw the birth of the modern gay rights movement in New Zealand, with the formation of Gay Liberation groups in Auckland, Wellington, and Christchurch (Ministry for Culture and Heritage, 2014). The first bill to propose the decriminalisation of homosexual acts, introduced in 1974, was unsuccessful. ${ }^{8}$ Over a decade later the Homosexual Law Reform Bill was introduced, a private member's bill which proposed to decriminalise sex between men over the age of 16 , and outlaw discrimination based on sexual orientation in employment, accommodation, and the supply of goods and services (Rishworth, 2007). The bill was highly controversial but was passed in $1986 .{ }^{9}$ The section of the bill proposing the removal of sexual orientation-based discrimination was, however, rejected.

Discrimination on the basis of sex and sexual orientation was later outlawed with the Human Rights Act (1993). The Act does not specifically prohibit discrimination on the basis of gender identity and expression, although the Human Rights Commission (2010) states that the Human Rights Act (1993) makes it unlawful to discriminate on the basis of gender identity. There is ongoing concern, however, that

\footnotetext{
${ }^{7}$ An agreement to found a nation state and build a government in New Zealand signed by representatives of the British Crown and Māori leaders.

${ }^{8}$ Perhaps in part because gay rights organisations refused to support a bill which set the age of consent for homosexual sex at 21, rather than 16, as was the age of consent for heterosexual sex (Dorey, 2006).

${ }^{9}$ The Government has since issued an official apology for convictions for historical 'homosexual offenses', and in 2017 a bill was introduced to allow men with historical convictions to apply to erase these convictions from their record.
} 
the law as it stands does not protect trans people from discrimination who have not, or will not, have gender-affirming surgery (Polster, 2003).

\section{Recognition of queer relationships}

The Civil Union Act (2004), along with the Relationships (Statutory References) Act (2005), established civil unions for both same-sex and opposite-sex couples, and gave all couples in New Zealand (including married couples, or those in a civil union or de facto relationship) roughly the same rights and responsibilities. Couples in civil unions were not, however, able to jointly adopt children, as married couples could (Rishworth, 2007). In 2012, Labour Party MP Louisa Wall introduced a bill which proposed to redefine marriage as the union of two people "regardless of sex, sexual orientation, or gender identity" (p. 2). The Marriage (Definition of Marriage) Amendment Act (2013) was passed in 2013, making New Zealand the $13^{\text {th }}$ country in the world to achieve marriage equality.

\section{Trans and intersex rights}

In New Zealand, trans people can access gender-affirming healthcare through the public health system (McDonald \& Byrne, 2015). Funding for genital surgeries is provided by the Ministry of Health's special high cost treatment pool, a fund set aside for one-off treatments not otherwise publicly funded (Ministry of Health, 2018a). Those in need are referred by a specialist to a waitlist, either for masculinising or feminising surgery. ${ }^{10}$ In 2004 a cap for the number of funded surgeries was introduced (one masculinising surgery and three feminising surgeries every two years), and as of October 2018 the wait list for surgery was over 50 years long (Ministry of Health, 2018c). In October 2018, the Government removed the cap on the number of surgeries performed, while the previous cap became a minimum number of surgeries every two

\footnotetext{
${ }^{10}$ Gender-affirming genital surgeries encompass a range of procedures, which fall into two groups. The Ministry of Health has previously used the terms "male to female" and "female to male" as shorthand to differentiate these groups of surgeries, as well as their waitlists (Ministry of Health, 2018b, 2018c). I avoid the use of these terms as they reify myths of medical transition as 'switching' from one gender to another. Instead, I adopt the terms "feminising surgery" and "masculinising surgery". While the terms"feminising" and "masculinising" are an improvement over "male to female" and "female to male" I acknowledge that they are not entirely unproblematic, as they imply that gender is associated with particular kinds of bodies.
} 
years (Coughlan, 2018). It remains unclear, however, how many genital surgeries the Ministry of Health will now fund.

Other gender-affirming healthcare services are provided by local DHBs (Ministry of Health, 2018a), however there is a lack of information regarding the current availability of these various services. In 2018 my colleagues and I requested information from each of the 20 DHBs about the gender-affirming healthcare services they provide and found remarkable inconsistency regarding which services were available and funded (Fraser, Shields, Brady, \& Wilson, 2018). Trans people report widespread knowledge gaps among practitioners regarding trans healthcare in New Zealand (Wi-Hongi, Greig, \& Hazenburg, 2017).

As well as uncertainty regarding the availability of gender-affirming healthcare services, there was a lack of clarity when I began my research regarding the process of accessing these services, largely due to the absence of national guidelines for healthcare providers. ${ }^{11}$ An assessment by a mental health professional is routinely required to access gender-affirming healthcare, however there is a lack of consensus on what the assessment should contain, or who is qualified to carry it out (Wi-Hongi et al., 2017). I explore models of gender-affirming healthcare in Chapter 7 (see pages 127-128) and consider the role of the mental health professional in providing this care.

With regard to legal documentation, trans people in New Zealand are permitted to change their gender on their birth certificate if they can provide evidence that they have "acquired a physical conformation that accords with their gender identity" (Schmidt, 2015, para. 2). The Family Court, which considers applications for changing gender on birth certificates, notes that applicants must have had 'medical treatment' to change their gender. This does not necessarily mean genital surgery, however prior to 2008, changing one's gender on a New Zealand birth certificate was only permitted following gender affirmation surgery to alter one's genitals (Schmidt, 2015). Gender can be changed on New Zealand passports and driver licenses through a simple

\footnotetext{
${ }^{11}$ Oliphant and colleagues (2018) have since published Aotearoa New Zealand Guidelines for Gender Affirming Healthcare, which have not been formally endorsed by the Minstry of Health, but have been endorsed by the Australian and New Zealand Professional Association for Trans Health, the New Zealand Sexual Health Society, and the New Zealand Society of Endocrinology.
} 
statutory declaration (a written document completed in front of an authorised witness; Department of Internal Affairs, 2019; New Zealand Transport Agency, 2019).

Currently, 'normalising' medical interventions on intersex infants are legal in New Zealand (Asia Pacific Forum, 2016). Intersex community organisations in New Zealand are currently campaigning for legal reform that would criminalise deferrable medical interventions that alter the sex characteristics of infants and children without personal consent. I elaborate on the history of intersex medical interventions on page 18.

\section{Mental Health and Wellbeing in Rainbow Communities}

A substantial body of research documents disparities in mental health and wellbeing among sexuality, gender, and sex characteristic diverse people. Compared with their heterosexual counterparts, lesbian, gay, and bisexual people are (for example) at increased risk for depression (Cochran \& Mays, 2000; Marshal et al., 2011), anxiety (Sandfort, de Graaf, Bijl, \& Schnabel, 2001), disordered eating (Austin et al., 2009), substance abuse problems (Woody et al., 2001), suicidal ideation and attempts (Garofalo, Wolf, Kessel, Palfrey, \& DuRant, 1998), and engagement in selfinjury (Batejan, Jarvi, \& Swenson, 2015).

Meta-analytic studies highlight the magnitude of these mental health disparities. Using data from 214,344 heterosexual and 11,971 non-heterosexual people across 25 studies, King and colleagues (2008) found that lesbian, gay, and bisexual people were twice as likely as heterosexual people to attempt suicide, and 1.5 times more likely to meet criteria for depression, an anxiety disorder, or alcohol and other substance abuse

problems. Young rainbow people may be particularly at risk. Lucassen and colleagues' (2017) meta-analysis of population-based studies of youth wellbeing found that sexual minority youth have three times the odds of reporting depressive symptoms and depressive disorder when compared to their heterosexual peers.

Research in trans communities shows similarly concerning results. Rates of depression, anxiety, and other mental health problems among trans people far surpass those of cis people (Budge et al., 2013; Fredriksen-Goldsen et al., 2013). Results from the US Community Health Centre Core Data Project revealed that $29 \%$ of trans respondents reported a suicide attempt at some point in their lifetime (compared with 
8.5\% of cis participants; Reisner, White, Bradford, \& Mimiaga, 2014). As well as these mental health difficulties, trans people are also at an increased risk of self-injury (Liu \& Mustanski, 2012), HIV infection (Grant et al., 2010), and substance use (Bradford, Reisner, Honnold, \& Xavier, 2013). While previous studies focus primarily on the health and wellbeing of trans men and women, or often fail to differentiate between the experiences of binary and nonbinary trans people, the U.S. Trans Survey found that nonbinary people are more likely to report serious psychological distress than trans men and women (James et al., 2016).

There is little research on the health and wellbeing of rainbow people who fall outside of the 'LGBT umbrella' (including intersex, asexual, and pansexual people, as well as those who identify as queer or questioning), possibly as a result of the difficulties associated with recruiting minorities within minorities (see, for example, Leonard, Lyons, \& Bariola, 2015). Because many studies have small sample sizes of rainbow minority groups, researchers generally lack statistical power to conduct analyses of differences between smaller groups within this population, and often collapse these groups into an 'other' category (Blair, 2016). Available research, however, suggests that rates of mental health difficulties among pansexual, queer, and questioning people are comparable to those among lesbian, gay, and bisexual people (Brewster, Moradi, Deblaere, \& Velez, 2013; Mayock, Bryan, Carr, \& Kitching, 2008; Mustanski, Garofalo, \& Emerson, 2010).

Previous studies examining the health and wellbeing of asexual people show mixed results, with some finding mental health disparities between asexual and heterosexual people (Yule, Brotto, \& Gorzalka, 2013), and others finding no difference (Brotto, Knudson, Inskip, Rhodes, \& Erskine, 2010; Greaves et al., 2017). The few available studies examining the mental health of intersex people also report mixed findings. Hines, Ahmed, and Hughes (2003) report that women with complete androgen insensitivity syndrome (an intersex condition) did not differ for any psychological outcomes when compared to matched controls. In contrast, other studies indicate that intersex people score substantially lower than the population average on a measure of general health function (Pitts, Smith, Mitchell, \& Patel, 2006), and report higher levels of self-reported psychological distress (Schützmann, Brinkmann, Schacht, \& RichterAppelt, 2009). The mixed findings apparent in this body of literature highlights the need 
for more research on the health and wellbeing of rainbow people outside the 'LGBT umbrella'.

\section{Mental Health and Wellbeing in New Zealand's Rainbow Communities}

The limited research on the health and wellbeing of rainbow communities in New Zealand closely reflects international findings. Results from the Youth 2012 survey, a nationally representative study of the health and wellbeing of secondary school students in New Zealand, showed that the majority of sexual minority youth reported good general health, had caring friends, and liked school. LGB young people were, however, three times more likely to experience significant depressive symptoms when compared to their heterosexual peers and had higher prevalence estimates of selfharm and suicidality (Lucassen, Clark, Moselen, Robinson, \& Adolescent Health Research Group, 2014). Youth Wellbeing Study data supports these findings; in a sample of 1799 Wellington secondary school students, LGB young people were five times more likely to report lifetime engagement in self-injury than their heterosexual peers (Fraser et al., 2018). Results from the Dunedin Multidisciplinary Study and the Christchurch Health and Development Study, two large-scale birth cohort studies, also demonstrate increased rates of mental health difficulties and suicidality among sexual minority young adults (Fergusson et al., 1999, 2005; Skegg, Nada-Raja, Dickson, Paul, \& Williams, 2003).

New Zealand research reveals similar disparities in mental health and wellbeing between trans and cis people. When compared to their cis peers, trans youth in the Youth 2012 survey reported increased rates of depressive symptoms $(O R=5.7)$, attempted suicide $(O R=5.0)$, and self-harm $(O R=2.7$; Clark et al., 2014). While no comparative study between trans and cis adults has been conducted in New Zealand, findings from Counting Ourselves (the first health survey for trans and nonbinary people living in Aotearoa New Zealand; Veale et al., 2019) showed that $71 \%$ of participants reported high or very high psychological distress, compared with $8 \%$ of adults in the New Zealand Health Survey (Ministry of Health, 2017). 


\section{Historical Understandings of Rainbow Mental Health}

Understandings of the high rates of mental health difficulties among rainbow community members have shifted drastically over the last century. In this section I provide a brief overview of the historical pathologisation of rainbow identities within the psy* disciplines (i.e., psychiatry, psychology, psychoanalysis, and psychotherapy). I do so because for those of us working in the field of mental health, this is our whakapapa. It is essential to acknowledge the history of our field, as this shapes contemporary understandings of rainbow mental health, and continues to impact the lives of queer, trans, and intersex people.

\section{The Pathologising Perspective}

For the greater part of the $20^{\text {th }}$ century, the psychological literature was dominated by research that pathologised sexuality, gender, and sex characteristic diverse people (for historical review, see Morin, 1977; Pettit \& Hegarty, 2014). According to this view, being heterosexual and cis $^{12}$ was the biological norm, and rainbow identities were considered diseases or disorders resulting from internal defects or external causes, such as inadequate early parenting (e.g. Bieber et al., 1962; Radó, 1940). The quote below from Edmund Bergler voices an attitude common amongst psychoanalytic theorists of the 1950s (Drescher, 2008):

I have no bias against homosexuals; for me they are sick people requiring medical help . . . Still, though I have no bias, I would say: Homosexuals are essentially disagreeable people, regardless of their pleasant or unpleasant outward manner ... [their] shell is a mixture of superciliousness, fake aggression, and whimpering. Like all psychic masochists, they are subservient when confronted with a stronger person, merciless when in power, unscrupulous about trampling on a weaker person (Bergler, 1956, p. 28-29).

\footnotetext{
12 Though, given that this term was not in use until the 1990s (Cava, 2016), it may be more accurate to say that not being trans was the biological norm.
} 
Similar attitudes were echoed by mental health professionals outside of the psychoanalytic tradition. For example, the influential psychiatrist Karl Menninger (1963) stated that "whatever [homosexuality is] called by the public, there is no question in the minds of psychiatrists regarding the abnormality of such behaviour" (p. 7). Indeed, conversion or reparative therapy - the practice of attempting to change an individual's sexual orientation or gender using aversive conditioning techniques (e.g. nausea-inducing medication or electric shocks), institutionalisation, castration, or talking therapies - was routinely practiced before the 1970s (Haldeman, 1994). The practice of conversion therapy has become increasingly less common over time, but it is still practised today, particularly in religious communities (Drescher et al., 2016). ${ }^{13}$

Since the 1950s, standard care for intersex infants has involved medical intervention (surgical, hormonal, or other interventions) intended to 'normalise' genitalia and other sex characteristics (Karkazis, 2008). Intersex medical interventions are rarely life-saving procedures or carried out to improve biological function; rather, they serve cosmetic and social needs (Reis, 2019). Such interventions, first led by the infamous psychologist John Money, are thought to increase intersex peoples' quality of life by facilitating a stable gender identity (Germon, 2009). Although performing unnecessary medical interventions on infants violates basic ethical principles of respect, autonomy, and informed consent (Reis, 2019), and have caused significant harm to intersex people and their families (Karkazis, 2008), normalising intersex medical intervention is still standard practice in many places around the world (Lee et al., 2016).

\section{Sexuality, Gender, and the DSM}

Pathologising understandings of rainbow identities have been (and, arguably, continue to be) enshrined in the DSM - often called "the bible of psychiatry" (e.g. Halpern, 2011, p. 488). Homosexuality was included in the first two editions of the DSM as a 'sociopathic personality disturbance' (APA, 1952) and a 'sexual deviation' (APA, 1968). In 1973, following the emergence of the gay liberation movements in the

\footnotetext{
${ }^{13}$ Conversion therapy is, at the time of writing, legal in New Zealand. A bill to prohibit sexual orientation and gender conversion therapy was introduced to Parliament in October 2018. The report of the New Zealand Parliament Justice Committee (2019) did not recommend an immediate ban, and noted that "we believe more work needs to be done before any decision is taken to ban [converstion therapy]" (p. 5).
} 
United States and protests by gay activists at the 1970 and 1971 annual APA meetings, the APA voted to remove homosexuality from the DSM-III (Gittings, 2008).

Evelyn Hooker and Alfred Kinsey's research findings were also influential in the APA's decision. Hooker's (1957) ground-breaking study showed that experienced psychologists could not differentiate between heterosexual and homosexual participants on the basis of several psychological tests, challenging the view that homosexuality was consistently associated with mental illness. Similarly, Kinsey, Pomeroy, and Martin's $(1948,1953)$ studies of human sexuality suggested that sexuality exists on a continuum, and that same-sex attraction is far more common than previously thought. Together, these findings added to a growing body of literature indicating that non-heterosexuality is part of usual human variation (Bene, 1965; Ford \& Beach, 1951; Freund \& Pinkava, 1961; McCord, McCord, \& Thurber, 1962).

The APA's decision to remove homosexuality from the DSM marked a turning point in understandings of mental health difficulties among rainbow people. It is important to note, however, that pathologising attitudes towards homosexuality persisted in the psy* disciplines. Many in the psychoanalytic community objected to the APA's decision, and petitioned for a referendum of the entire APA membership to determine support for removing homosexuality from the DSM. The decision was upheld, with 58\% of the APA's 10,000 (primarily North American) members voting in support of the decision (Kameny, 2009). Despite this, homosexuality lingered in the DSM in various forms ('sexual orientation disturbance' and 'ego-dystonic homosexuality') until its complete removal from the DSM-III-R in 1987 (Conrad \& Angell, 2004).

In many ways, the inclusion of gender-related diagnoses in the DSM parallels the earlier inclusion of diagnoses related to sexual orientation. Prior to the publication of the DSM-V, the APA held the position that identifying with a gender that differed from one's sex assigned at birth was evidence of a mental disorder (Cohen-Kettenis \& Pfäfflin, 2010). Trans identities first appeared in the DSM-III as 'gender identity disorder of childhood' and 'transsexualism' (APA, 1980). A third diagnosis, 'gender identity disorder of adolescence and adulthood, nontranssexual type' was added in the DSM-III-R. In the DSM-IV, this last disorder was removed, and the previous two combined into 'gender identity disorder' (APA, 1994 
The inclusion of 'transsexualism' and 'gender identity disorder' in the DSM was heavily criticised as pathologising and stigmatising by researchers, mental health practitioners, and trans community members (Cohen-Kettenis \& Pfäfflin, 2010; Kamens, 2011; Lev, 2005). In response to these concerns, the American Psychological Association formed the Task Force on Gender Identity and Gender Variance, to consider revision of this diagnosis in the DSM-V. The report of the task force (American Psychological Association Task Force on Gender Identity and Gender Variance, 2008), together with later APA reports and position statements, signals a dramatic shift in both associations' positions on gender diversity. The 2012 APA Position Statement on Discrimination stated that "being trans or gender variant implies no impairment in judgment, stability, reliability, or general social or vocational capabilities" (p. 1) and emphasises that trans people often experience discrimination based on their gender identity or expression (Drescher, Haller, \& American Psychiatric Association Caucus of Lesbian, Gay and Bisexual Psychiatrists, 2012).

Although many trans people and organisations called for the APA to remove gender identity disorder from the DSM entirely (Ault \& Brzuzy, 2009; Bouman, Bauer, Richards, \& Coleman, 2010; Cohen-Kettenis, 2001), the World Professional Association for Trans Health (WPATH) expressed concerns that the removal of a diagnosis related to gender identity disorder would threaten trans people's access to healthcare and support services. WPATH did, however, recommend changes in name and placement within the DSM, and advocated for diagnostic criteria based on distress, rather than identity (Knudson, De Cuypere, \& Bockting, 2010). The APA subsequently replaced 'gender identity disorder' with 'gender dysphoria' in the DSM-V (APA, 2013), emphasising that, although they are related, gender dysphoria is distinct from trans experiences. As such, the DSM criteria no longer applies to all trans people, but only to those who experience distress related to the incongruence between their assigned sex and gender.

While the APA was widely applauded for opposing discrimination against trans people and supporting their access to care, the decision to include gender dysphoria in the DSM-V was not without controversy. Davy (2015) argues that the change from gender identity disorder to gender dysphoria continues to stigmatise trans people and suggests that an emphasis on distress may encourage trans people to "frame their 
narratives in line with the distress model in the DSM-5" (p. 1166; I return to this point in Chapter 7 in my discussion of the 'trans narrative'). Notably, the APA has retained other pathologising diagnoses in the DSM-V which have failed to attract the widespread attention of the gender dysphoria diagnosis (e.g. 'transvestic disorder'). As such, it is perhaps premature to describe the pathologisation of rainbow identities as entirely 'historical' within psychology and psychiatry.

\section{Contemporary Understandings of Rainbow Mental Health}

\section{The Minority Stress Perspective}

Rainbow mental health disparities are now predominantly understood using the minority stress perspective, which proposes that societal stigma and discrimination create a hostile and stressful environment for rainbow community members. This stress, in turn, increases risk for developing mental health problems (Brooks, 1981; Meyer, 1995). Meyer (2003) posited that minority stress is unique to minority groups (that is, additive to general stressors experienced by all people), chronic, and is based in social processes, institutions, and structures. As such, Meyer (2003) challenged pathologising perspectives that locate the causes of mental health difficulties within the individual. The minority stress model describes several 'stress processes' relevant to LGB people: (1) external stressful events, such as discrimination and violence; (2) expectations of discrimination and rejection, and the vigilance that comes with this expectation; (3) hiding or concealing sexual orientation; (4) internalising negative societal attitudes; and (5) ameliorative coping processes.

The minority stress model is supported by a substantial body of empirical evidence. Rainbow community members are disproportionately exposed to discrimination and violence (Friedman et al., 2011; Mays \& Cochran, 2001). Moreover, victimisation based on sexual orientation and gender is associated with depression, attempted suicide, and health risk behaviours (Bontempo, And, \& D'augelli, 2002; Russell, Ryan, Toomey, Diaz, \& Sanchez, 2010). Perhaps the strongest evidence for the minority stress model comes from studies using mediation analyses, which show that experiences of discrimination account for the relationship between LGBT status and negative mental health outcomes (such as depressive symptomatology or suicidality; 
Almeida, Johnson, Corliss, Molnar, \& Azrael, 2009; Toomey, Ryan, Diaz, Card, \& Russell, 2010).

The minority stress model implies two key points of intervention in addressing the high rates of mental health problems among rainbow people. Meyer (2003) labelled these the "subjective view" and the "objective view" (p. 22) and suggested that both types of intervention can result in a reduction in mental health problems. The objective view highlights the role of environmental stressors, and points to interventions that aim to reduce societal stigma and prejudice. As such, researchers in this area often highlight the need for structural change; policy initiatives, education, and funding to reduce bullying, harassment and discrimination (e.g. Burton, Marshal, Chisolm, Sucato, \& Friedman, 2013; Hatzenbuehler, 2010; James et al., 2016). The subjective view focuses on individual processes and suggests that individual interventions "should aim to change the appraisal process, the person's way of evaluating their condition and coping with stress and adversity" (Meyer, 2003, p. 24).

Meyer's (2003) minority stress model has been critiqued for reducing stress to individuals, and for failing to account for the role of social norms and institutions (Riggs \& Treharne, 2017). Riggs and Treharne argue that ideologies (rather than individuals) are productive of stress, and that ideologies work in concert with one another. As such, we do not experience our identities as individual categories, but as multiple and overlapping. Meyer, however, restricted his focus to sexual orientation, and failed to consider whether the effects of prejudice are uniform across all LGB people. Accordingly, Riggs and Treharne highlight the need for an intersectional focus when thinking about stress and suggest that the concept of privilege is of use to intersectional accounts of stress.

Riggs and Treharne (2017) have identified some clear gaps in Meyer's (2003) minority stress model. In particular, the minority stress model would benefit from acknowledging the intersectional nature of identity and marginalisation, and from drawing on the concept of privilege to account for individual differences in experiences of stress and coping among marginalised group members. In saying this, destabilising societal institutions, norms, and ideologies is likely to be a slow process, and in the meantime, many members of rainbow communities need support. Because of this, it seems that Meyer (2003) rightly asserted that addressing mental health disparities in 
rainbow communities requires intervention at the individual level, to encourage resilience building, and to provide a space for processing experiences of discrimination and violence.

\section{Conclusion}

The history of rainbow rights in New Zealand shows significant progress toward making Aotearoa a safe and inclusive place for sexuality, gender, and sex characteristic diverse people. However, ongoing rainbow mental health disparities demonstrate that there is still much work to be done in this area. Developing an understanding of rainbow community members' experiences in mental health settings is essential to identify potential areas for improvement in clinical training and practice. In the next chapter I review empirical studies on rainbow peoples' experiences of accessing mental health support and consider how my research can address gaps in this literature to inform the provision of mental health support for Aotearoa's rainbow people. 


\section{Chapter 3: Literature Review}

In this chapter, I review the literature on rainbow peoples' experiences of accessing mental health support. First, I identify common elements of negative and positive experiences across previous studies and examine the experiences of particular groups within the rainbow community. I then review research on rainbow mental health support experiences conducted in the New Zealand context. I conclude this chapter by reflecting on the discrepancies apparent in this body of research and considering the ways in which this thesis can help to bridge gaps in the literature.

\section{Rainbow Experiences of Accessing Mental Health Support}

Studies examining rainbow peoples' experiences of accessing mental health support have produced varied results. While the majority of studies in this body of literature focus on the negative experiences of rainbow community members in mental health settings (e.g. Bowers, Plummer, \& Minichiello, 2005; Lucksted, 2004; Nystrom, 1997; Page, 2004; Poteat, German, \& Kerrigan, 2013), others report that many queer and trans people find their mental health professionals helpful, and have positive experiences when accessing support (Hunt, Matthews, Milsom, \& Lammel, 2006; Israel, Gorcheva, Burnes, \& Walther, 2008; Ross, Law, \& Bell, 2016; Willging, Salvador, \& Kano, 2006). As such, there is no consensus what accessing mental health support is like for rainbow people.

The varied findings across this body of literature (and, indeed, in any body of literature on client experiences in mental health settings) are to be expected given that rainbow experiences of mental health support most likely depend on a wide range of factors, including client characteristics, professional characteristics, as well as physical and temporal context. Moreover, there is a dearth of representative research in this area. This is partially because many studies in this body of literature are qualitative (Filice \& Meyer, 2018), so are not designed to be generalisable to the wider rainbow community. On top of this, researchers working with rainbow communities tend to rely on convenience or snowball sampling; rainbow community members are relatively more 
hidden than members of other minority groups, so researchers typically recruit participants from existing social networks, or through queer and trans community organisations and venues (Cochran, 2001).

Although this body of literature does not present a cohesive picture, common threads of experience are apparent across studies. In this review, I explore the elements of mental health support experiences that sexuality, gender, and sex characteristic diverse clients commonly report as negative versus positive. For the most part, I avoid attempts to quantify negative versus positive experiences of mental health support in rainbow communities. Before I begin, I note two key methodological considerations.

\section{Methodological Considerations}

Firstly, in this review I focus on people's experiences accessing mental health support (from psychologists, psychiatrists, counsellors, therapists, and other mental health professionals) rather than physical healthcare (though there is a growing literature on queer, trans, and intersex peoples' experiences of accessing physical healthcare; see, for example, Beehler, 2001; Hoffman, Freeman, \& Swann, 2009; Neville \& Henrickson, 2006). Where studies conflate mental and other health professionals, I use "health professionals" or "experiences in healthcare" to signal this.

Secondly, research in this area varies in terms of the population under study. The research I review in this chapter includes previous studies specific to gay men, lesbians, gay men and lesbians, LGB people, LGBT people, LGBQ people, LGBTQ people, bisexual people, trans men, and trans people. In the first section of this review I summarise all of this research together, as there is considerable overlap in mental health support experiences across groups. I then consider patterns of experiences among bisexual people and trans people, as it became apparent when reviewing the research literature that these groups face unique challenges in mental health settings.

\section{What Makes a Negative Experience?}

Throughout the literature, rainbow clients of mental health services describe experiences as negative when their mental health professional expressed or perpetuated homophobia, transphobia, heterosexism, and/or cissexism. Clients also commonly describe receiving poor or inadequate service as a result of a lack of provider 
knowledge about sexuality, gender, and sex characteristic diversity. In some studies, these negative experiences are conceptualised as existing on a continuum. For example, in a 2004 report, Lucksted refers to "The Continuum from Ignorance to Discrimination" (p. 19), and argues that although ignorance can be unintentional (e.g. if a professional's training programme or workplace has not provided them with relevant information), a lack of knowledge on the part of mental health professionals can, and does, have similar negative impacts to a client's ability to recover as does overt prejudice.

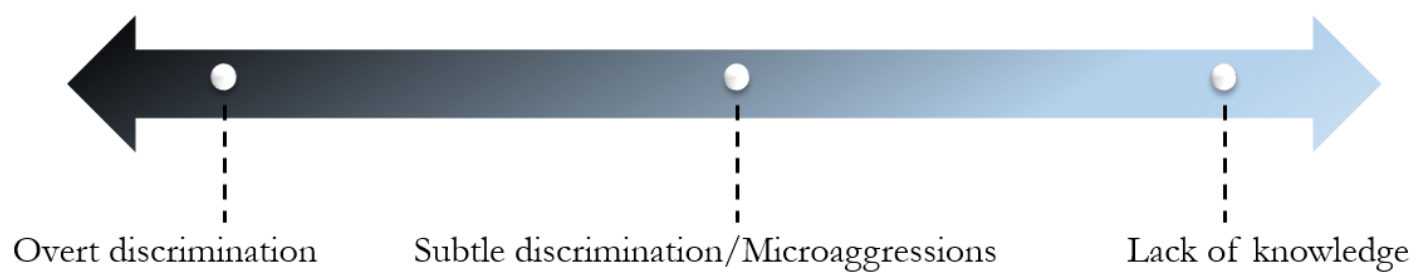

Figure 1. Graphical depiction of Lucksted's continuum of negative experiences.

Here I use Lucksted's framework (depicted in Figure 1) to structure a review of rainbow clients' negative experiences in mental health support settings, and to consider the ways in which these experiences may have changed over time. I begin by reviewing the most overt examples of harassment and discrimination described within the literature, before outlining more subtle discrimination or microaggressions, and finally poor service as a result of ignorance or knowledge gaps. While I do my best to differentiate between these three types of experiences, the boundaries between them are unclear. As such, my classification of some experiences differs from that of the cited authors. $^{14}$

\section{Overt discrimination}

Reports of overt discrimination are common across studies in this body of literature. In Nystrom's (1997) survey, almost one quarter of the 610 gay men and

\footnotetext{
${ }^{14}$ For example, Shelton and Delgado-Romero (2011) describe professionals' assumption that LGBQ individuals need psychotherapeutic treatment as a microaggression, whereas I classify this as overt discrimination.
} 
lesbians who had accessed mental health support reported they had received poor or inappropriate service because of their sexual orientation. Nearly three quarters of 55 LGB adults in Golding's (1997) study reported experiences of prejudice or violence within mental health services, while in a survey of 116 LGB clients, $64 \%$ had experienced discrimination or poor treatment in the mental health system because of their sexual orientation (Lucksted, 2004).

Perhaps the most comprehensive account of LGBT discrimination experiences within mental health settings is seen in Lucksted's (2004) aforementioned report, which identified evidence of lingering pathologisation of LGBT identities within mental health services. Altogether, Lucksted contacted over 500 people and organisations in the course of her multi-year project. She reviewed academic literature, grey literature, and conducted informal interviews with more than 30 key informants, including LGBT consumers, activists, community workers, and/or service providers in mental health settings. LGBT clients of mental health services reported that they had been denied services because of their gender or sexuality, being unnecessarily physically restrained in inpatient settings, and that professionals had assumed they were sexual predators on the basis of their sexual orientation. Lucksted concluded that many mental health professionals seemed to equate 'health' with conventional heterosexual presentation and saw their clients' sexuality as an aspect of their mental illness, rather than as a potential area of strength, resilience, or positivity in their life.

The discriminatory experiences outlined in Lucksted's (2004) report reflect those reported elsewhere in the literature. Other specific examples of overt discrimination include instances of mental health professionals pathologising clients' sexual orientation (Eady, Dobinson, \& Ross, 2011; King et al., 2003; Liddle, 2000; Shelton \& Delgado-Romero, 2011), verbally harassing clients as a result of their sexuality or gender identity (Liddle, 1996; Nystrom, 1997), disparaging clients' "lifestyle choice" (Willging et al., 2006, p. 868), advising clients that ending a queer relationship would alleviate their psychological distress (King et al., 2003), discouraging clients from sharing their identity with others (Israel et al., 2008), refusing to use correct pronouns when referring to a client (McCullough et al., 2017), offering conversion therapy or encouraging clients to 'renounce' their sexual orientation (Bartlett, Smith, \& King, 2009; Israel et al., 2008; Liddle, 2000; McFarlane, 1998; 
Nystrom, 1997), denying the reality of a client's sexual orientation or dismissing it as a fad or phase (McFarlane, 1998; Nystrom, 1997; Platzer, 2006), or searching for a cause of a non-heterosexuality, such as childhood sexual abuse (McFarlane, 1998; Platzer, 2006). A small minority of participants in Nystrom's (1997) study reported inappropriate sexual behaviour by their therapist.

While the studies in the above paragraph focus predominantly on sexual orientation, rather than gender, trans clients have also reported that their gender identity was treated as a symptom of mental ill health, rather than being validated as genuine (Ellis, Bailey, \& McNeil, 2015), and that they have experienced verbal abuse, or been denied treatment, as a result of their gender identity (Pitts et al., 2009). I elaborate on experiences specific to trans clients later in this chapter.

\section{Subtle discrimination and microaggressions}

As well as overt discrimination, it is important to acknowledge the ways in which more subtle forms of discrimination, such as microaggressions, may impact on rainbow experiences within mental health support settings. In a 2011 study of LGBQ clients' psychotherapy experiences, Shelton and Delgado-Romero identified a number of microaggressions specific to sexual orientation, including assumptions that sexual orientation is the cause of all presenting issues, attempts to over-identify with LGBQ clients (e.g. professionals mentioning their LGBQ friends or acquaintances in session), making stereotypical assumptions about LGBQ clients, and displaying books, brochures and pamphlets that ignore LGBQ issues and individuals. Participants also reported that professionals maintained a silence around sexuality. Issues pertaining to sexual orientation were often minimised or avoided altogether, with participants recounting instances of their therapists avoiding the use of LGBTQ terminology or making statements such as "You don't need to worry about [your LGBQ identity] right now" (p. 214).

Reports that mental health professionals are silent about gender and sexuality, or tend to minimise the importance of identity in clients' lives, are echoed across other studies. In Daley's (2010) study of self-disclosure of lesbian or queer identity in mental health settings, some participants reported that they were not given a space to explore or discuss their identity when involved with mental health services. Similarly, some participants in Kidd, Veltman, Gately, Chan, and Cohen's (2011) study of lesbian, gay, 
and trans individuals with severe mental illness described providers ignoring their sexual orientation, or not allowing for conversation about queer relationships. In contrast, LGBT participants in Israel and colleagues' (2008) study reported that they found it unhelpful when therapists focussed on their sexual orientation or gender identity inappropriately. As such, an overemphasis on identity may be as unhelpful as a lack of emphasis on identity.

Another microaggression reported by queer and trans clients across studies is mental health professionals making assumptions about their clients' identities (Eady et al., 2011; Hunt et al., 2006; Liddle, 1996; Robertson, 1998; Saulnier \& Wheeler, 2000). Professionals commonly communicate these assumptions through use of gendered language. For example, a mental health professional may ask a male client if they have a girlfriend, rather than a partner, forcing the client to either come out in response (potentially before they are ready), or to remain silent about their identity (Robertson, 1998). Assumptions about clients' gender and sexuality are also communicated when mental health professionals respond to clients' disclosures of identity with surprise or discomfort (Eady et al., 2011; Evans \& Barker, 2010; McCullough et al., 2017; Saulnier, 2002).

\section{Lack of knowledge}

Perhaps the most common negative experience reported by rainbow people is a lack of provider knowledge or competence regarding sexuality, gender, and sex characteristic diversity. Rainbow people report a lack of knowledge about the differences between sexuality, gender, and sex characteristics, the identity terms clients use, aspects of rainbow culture, the difficulties associated with coming out, and the complex ways in which societal pressures and discrimination impacted on clients' mental health problems (Hunt et al., 2006; Lucksted, 2004; McCullough et al., 2017; Poteat et al., 2013; Shelton \& Delgado-Romero, 2011).

Rainbow people consistently report that they have to educate their mental health professional in order to access the care they needed (Bauer et al., 2009; Corliss, Belzer, Forbes, \& Wilson, 2007; Evans \& Barker, 2010; Grove, 2009; Kidd et al., 2011; Lucksted, 2004; McCann \& Sharek, 2014; McIntyre et al., 2011), with some even providing reading material to their mental health professionals (Hunt et al., 2006). The need to educate mental health providers undermines the trust of the therapeutic 
relationship, and can be a frustrating, alienating, and exhausting process for queer and trans clients (Eady et al., 2011; Lucksted, 2004; McCullough et al., 2017). When mental health professionals have a poor understanding of their client's day-to-day experiences as a rainbow person, this forms a significant barrier to recovery in mental health support settings, especially in supporting clients through identity development (Hunt et al., 2006; Mair, 2003).

\section{What Makes a Positive Experience?}

Unsurprisingly, the factors that make a mental health support experience positive are, for the most part, the inverse of those that make an experience negative. Across the literature, rainbow participants most commonly emphasised the importance of mental health professionals treating their clients with respect, affirming and validating their identities, and being knowledgeable about sexuality, gender, and sex characteristic diversity (Burckell \& Goldfried, 2006; Israel et al., 2008; Page, 2004; Rachlin, 2002; Ross et al., 2016; Saulnier \& Wheeler, 2000; Scherrer, 2013). Queer and trans clients found mental health professionals helpful when they were caring, openminded, and non-judgemental (Corliss et al., 2007; Dobinson \& Macdonnell, 2005; Pitts et al., 2009).

Examples of good practice cited in the literature include providers asking openended questions, using neutral language to avoid making assumptions, helping to explore topics clients raise as important (including exploring identity), promoting client autonomy, ensuring clients have good support systems in place, matching clients' language use, and providing practical support when needed, such as support letters for gender-affirming healthcare (Corliss et al., 2007; Dobinson \& Macdonnell, 2005; Dorland \& Fischer, 2001; Eady et al., 2011; Israel et al., 2008; Mair, 2003; Ross et al., 2016). LGBT participants in Israel and colleagues' (2008) study described concrete ways that mental health professionals can communicate support and understanding, such as displaying small visual cues (e.g. LGBT supportive stickers and posters). In the same study, participants found it helpful when professionals supported them to come out to others, focussed on sexuality or gender when clients felt it relevant, and were knowledgeable about LGBT issues. 
Previous research suggests that queer clients appreciate professionals' efforts to explicitly challenge heteronormativity in their practice. In a mixed methods study of experiences in gay-affirmative therapy, gay and lesbian clients found it affirming when their counsellors communicated that they did not see their identity as a problem but understood that societal stigma was an ongoing problem (Pixton, 2003). Clients also found it affirming when they were given a safe and supportive space to discuss or explore their sexuality and found it useful when counsellors were aware of issues affecting gay and lesbian people. Finally, clients found it affirming when counsellors used a holistic perspective, viewing their sexuality as a part of who they were, rather than segregating this from them as a person.

Finally, literature on client-therapist matching highlights that some factors influencing perceived helpfulness are outside of mental health professionals' control. In a number of studies, queer and trans clients report a preference for working with mental health professionals who are part of the rainbow community, as they found them more knowledgeable and understanding than mental health professionals outside of the rainbow community (Hunt et al., 2006; Jones, Botsko, \& Gorman, 2003; Ryden \& Loewenthal, 2001). Findings about the importance of client-therapist matching are equivocal, however, with participants in other studies emphasising the importance of therapist competence over identity (Burckell \& Goldfried, 2006; Liddle, 1996; Saulnier, 2002). As such, it is likely that the extent to which rainbow clients prefer rainbow therapists largely reflects individual differences (Burckell \& Goldfried, 2006).

\section{The Impact of Negative Therapeutic Experiences}

Negative mental health support experiences have significant implications for the mental health and recovery of rainbow community members. Queer and lesbian women in Daley's (2010) study described their attempts to adhere to heteronormative requirements in mental health service settings as contributing to their mental health difficulties, and as a barrier to recovery, while Lucksted (2004) notes that homophobic experiences "impede recovery and exacerbate existing problems by increasing stress and conflict in settings where clients expect support" (p. 26). Negative experiences can also alter clients' views of therapy, reducing the likelihood that they will return (Shelton \& Delgado-Romero, 2011). In contrast, clients who feel supported in mental health 
settings are subsequently more likely to reach out for support (Dorland \& Fischer, 2001; Goettsche, 2015).

The potential for a negative experience in a mental health support setting also influences how comfortable clients feel about coming out in therapy. In numerous studies across this body of literature participants found disclosing their identity in mental health settings stressful and anxiety-provoking, as they were unsure about how their mental health professional would respond; participants described fears that their mental health professional would lack understanding, or respond with surprise, discomfort, or discrimination (Daley, 2010; Dobinson \& Macdonnell, 2005; Evans \& Barker, 2010; Hiestand, Horne, \& Levitt, 2007; Hunt et al., 2006; Robertson, 1998; Semp, 2006; Shelton \& Delgado-Romero, 2011; Sperber, Landers, \& Lawrence, 2008). This fear is not universal: many rainbow people feel confident and comfortable coming out to their mental health professional (Daley, 2010), or only feel it is necessary to disclose their identity if it is important in treatment (Hunt et al., 2006). For other rainbow community members, however, coming out in therapy is essential, not only because identity is important to consider in relation to mental health and emotional wellness, but because they feel that mental health professionals need to know about their identity in order to view them as a whole person (Dobinson \& Macdonnell, 2005; Hunt et al., 2006; Lucksted, 2004).

\section{Experiences of Groups Within the Rainbow Community}

In the previous section I identified common threads throughout the literature on rainbow experiences of mental health support. While there is a great deal of overlap in experience within the rainbow community, it is important to acknowledge the diversity of queer, trans, and intersex people. In this section, I review literature relating to the unique experiences of bisexual and trans people who access mental health support.

\section{Bisexual experiences}

Research in queer mental health suggests that bisexual people may be at higher risk for poor health and wellbeing when compared to gay and lesbian people (for a review, see Dodge \& Sandfort, 2007). The increased risk of mental health difficulties among bisexual people is generally understood as a result of biphobia. Similar to homophobia, biphobia consists of negative attitudes towards bisexual people, or "the 
denigration of bisexuality as a valid life choice" (Bennett, 1992, p. 202). Unlike homophobia, however, bisexual people may experience rejection from both straight and gay communities (Ochs, 1996). Biphobia encompasses overt prejudice, as well as inaccurate social messages about bisexuality, including beliefs that bisexuality does not actually exist, is a phase, or is a transitional state in which people are in the process of 'deciding' between a gay or straight identity (Mulick \& Wright, 2002).

The literature on bisexual experiences of mental health support reflects the wider biphobia literature, suggesting that bisexual people may experience unique challenges in mental health settings. A key issue for bisexual clients of mental health services may be disclosing their identity as bisexual, particularly if they are in a seemingly heterosexual relationship; Richters (1997) notes that many providers may have already made assumptions about a client's sexuality on the basis of their current relationship status. Indeed, in King et al.'s (2003) study, bisexual men and women were less likely to disclose their sexual orientation to their mental health professional than gay and lesbian participants. Moreover, bisexual women were less likely to report a positive reaction to coming out, when compared to gay and lesbian participants.

Bisexual clients commonly describe biphobic behaviour by mental health professionals. Participants in Dobinson and Macdonnell's (2005) study reported experiences of healthcare providers making inappropriate jokes and comments, asking invasive questions, or equating bisexuality with having multiple partners. Similarly, in a survey of 217 bisexual women and men, participants described invalidation of bisexuality as the most important problem they faced in being both a mental health consumer and a bisexual, with one participant commenting "I always felt like my therapist was humoring me when we discussed my bisexuality, like she thought it was a phase, despite that I've had long-term relationships with both sexes fairly consistently" (Page, 2004, p. 147). A final example comes from Pallotta-Chiarolli and Martin's (2009) survey of bisexual young people and youth service providers in Australia about their experiences. A number of bisexual young people reported that their school counsellors and other adults in school settings had encouraged them to remain closeted, or to pass as straight, rather than challenging the culture that made it difficult or unsafe for bisexual young people to disclose their sexual orientation to others. 


\section{Trans experiences}

While trans people commonly present to mental health services for the same reasons as cis people - that is, for support or treatment for mental health difficulties many trans clients also present to mental health services in order to access genderaffirming healthcare (Bockting, Robinson, Benner, \& Scheltema, 2004; Ellis et al., 2015). This is because medical standards of care (such as the widely used WPATH standards; Coleman et al., 2012) generally require trans people to be assessed by a mental health professional before accessing puberty blockers, hormones, and/or surgery.

The involvement of mental health professionals in the process of accessing gender-affirming healthcare complicates the experiences of support seeking for many trans people. Because a referral letter from a mental health professional is commonly required to access gender-affirming healthcare, mental health professionals are often viewed as gatekeepers by their trans clients (Lev, 2004; Sperber et al., 2008; Taylor, 2013). Trans people often describe this gatekeeping element as having a negative effect on their relationship with their health providers (Rachlin, 2002; Taylor, 2013). Indeed, clients may have to decide whether they will be open about their distress or emotional state, in the knowledge that this may impact on their access to gender-affirming healthcare. While "clinically significant distress" is required in order to meet the diagnosis of gender dysphoria (APA, 2013, p. 453), clients could be prevented from accessing gender affirming healthcare if they are too distressed, as a mental health professional may not consider their functioning as stable enough to start treatment (Coleman et al., 2012).

Within previous research on trans experiences of mental health assessments for gender-affirming healthcare, trans people commonly describe a need to conform to a dominant 'trans narrative' for fear that their actual trans experience may not be viewed as legitimate enough to access much-needed healthcare (Ellis et al., 2015; McNeil, Bailey, Ellis, Morton, \& Regan, 2012; Violeta \& Langer, 2017). This dominant narrative includes identifying as part of the gender binary, feeling 'trapped in the wrong body' from a young age, experiencing significant distress, and wanting a 'full' medical transition - that is, hormone treatment, as well as genital surgery (Davy, 2015; Lewis, Vincent, Brett, Gibson, \& Walsh, 2017). Many trans people express concern that they will not be referred for gender-affirming healthcare if they describe experiences that 
deviate from this narrative, such as feeling satisfied with one's genitals, having a nonbinary gender identity, or having identified as trans for a relatively short time (Davy, 2015; Rowe, 2014).

Trans people describe numerous examples of discrimination and invalidation within mental health settings, including mental health professionals asking invasive questions about their bodies and sex lives (Ellis et al., 2015; Pitts et al., 2009), refusing to treat them (Sperber et al., 2008), or discouraging them from expressing their gender (Corliss et al., 2007). Many health professionals seem to lack an understanding of why someone would want to medically transition; one participant in Taylor's (2013) study of trans mens' health care experiences reported being asked why they would not want to have a menstrual cycle. As a result of a widespread lack of knowledge and understanding, many trans people feel they must advocate for themselves in order to access care (Ross et al., 2016; Taylor, 2013). This includes networking with others in the trans community in order to find out who they can safely access healthcare from or visiting healthcare professionals armed with resources and information.

Trans people's experiences in gender identity clinics, or services specific to trans people, may differ to those within general healthcare services. For example, in Davies and colleagues' (2013) study of gender identity clinic services in the UK, 94\% of participants agreed that they would recommend the service to a friend or colleague if they had a gender-related problem. One participant commented "I appreciate how my clinician has always viewed the gender issue as being separate to the mental health issue and has always had faith in my willingness to be who I am" (p. 408). It is possible that mental health professionals in gender-specific services are knowledgeable about gender and trans issues, so are better equipped than professionals in general health services to support trans clients. Other studies, however, contradict these findings; nearly half of participants in Ellis and colleagues' (2015) study of gender identity clinics, also conducted in the UK, had experienced difficulties obtaining the treatment they needed, with participants reporting instances of being misgendered, and of clinicians adhering to rigid, binary understandings of gender. These inconsistencies suggest that, like professionals in general healthcare services, professionals in gender identity clinics might vary in their gender-related knowledge and experience. 


\section{New Zealand Research}

To date, three studies have specifically examined rainbow experiences of accessing mental health support in New Zealand. Welch and colleagues (2000) surveyed a non-representative sample of 561 lesbians, reporting that $80.7 \%$ of respondents had used a mental health service at some point in their lives. Of those who had accessed mental health services, $69.9 \%$ of respondents rated the last service they had used as very useful or somewhat useful, while nearly a third of those who had used mental health services had experienced treatment which they felt to be discriminatory. Discriminatory experiences closely reflected those seen in international literature, with providers focussing on sexuality when this was not relevant, not taking their clients' sexuality seriously, assuming heterosexuality, pathologising lesbianism, or suggesting that clients become heterosexual. Participants suggested that in order to improve mental health services for lesbian clients, mental health professionals should be given training about sexual orientation and issues of sexuality. Moreover, services should offer lesbian-friendly services, display posters aimed at lesbian clients, and make efforts to train and employ more lesbians.

The next study of queer experiences in New Zealand's mental health services was that conducted by Semp (2006). Semp identified discourses around homosexuality and mental health in New Zealand, then interviewed 13 men who have sex with men $(\mathrm{MSM})^{15}$ in Auckland who had accessed public mental health support, as well as 12 queer staff of public mental health services. Semp concluded that heteronormative discourses constrained the ways in which staff could work with queer clients in public mental health services. Staff in public mental health services rarely asked about sexual orientation and clients reported considerable difficulty discussing their sexuality, even in cases where it was relevant to their presenting difficulties. In order to disrupt this heteronormative silence, Semp argued that all staff in public mental health services should initiate conversations with their clients about sexuality. Moreover, Semp contended that matching queer clients and staff will not counter heteronormativity, as

\footnotetext{
${ }^{15}$ Semp's own framing. Semp noted that this behavioural description was important to reach men who engage in sexual activity with other men, regardless of how they identify themselves; many such men do not sexually identify as gay, homosexual or bisexual.
} 
matching incorrectly assumes similarity between identity groups, ignoring the diversity within queer communities.

A study initiated by the Auckland DHB followed Semp's foundational work (Birkenhead \& Rands, 2012). As part of this project, 20 queer and trans individuals and 47 service providers were interviewed. A survey of 106 rainbow service users of public mental health services in Central Auckland was also conducted via postal questionnaire. Overall findings showed that there are significant barriers to the delivery of responsive service provision, due to a lack of conversation around sexual orientation and gender. Many service users experienced heteronormativity in the approach of mental health professionals. However, there was significant variation in the experience of queer and trans clients; many service users found the experience positive and were comfortable discussing sexuality and gender in a mental health setting. The quality of care received seemed highly dependent on the skill and sensitivity of the clinician.

Couch and colleagues' (2007) Tranznation report on the health and wellbeing of trans people in Australasia provides further insight into trans New Zealanders' experiences in mental health settings. Of the 253 respondents to the Tranznation survey, $24(9.5 \%)$ were from New Zealand. Trans participants reported mixed experiences in healthcare settings. The best experiences were of being accepted and supported by practitioners, while the worst experiences involved instances of hostility and disrespect. The report did not differentiate between mental health professionals and physical health professionals and did not present results for New Zealand and Australian participants separately, so has limited utility for understanding the experiences of trans people in New Zealand's mental health support services. Results from the Tranznation report are, however, consistent with findings from the New Zealand Human Rights Commission Report of the Inquiry into Discrimination Experienced by Transgender People (2008), which described mixed experiences in general health services, and difficulties in accessing counselling or other support services.

A final study of note is Adams, Dickinson, and Asiasiga's (2012) assessment of the mental health promotion and suicide prevention needs of gay, lesbian, bisexual, transgender and intersex (GLBTI) populations in New Zealand. The project involved telephone or face-to-face interviews with 17 key informants (who either worked in GLBTI mental health or had an interest in the area) and online submissions by 124 
GLBTI people. The study report (Adams et al., 2012) and subsequent publications (Adams, Dickinson, \& Asiasiga, 2013a, 2013b; Dickinson \& Adams, 2014) identified three key themes: macro-social environment; social acceptance and connection; and services and support. In relation to services and support, Adams and colleagues (2012) reported that many respondents struggled to access services due to financial barriers and a lack of public mental health services. Respondents also emphasised the importance of rainbow cultural competency, and many reported interactions with mental health professionals who they found discriminatory, unsupportive, and lacking knowledge about rainbow issues and experiences. Practitioners' assumptions about their clients sexual identity contributed to many respondents' negative experiences, and some reflected on "the dangers of linking sexual identity with the pathology of mental health" (p. 53).

\section{Reflections on this Body of Literature}

Past research exploring rainbow experiences of accessing mental health support suggests that the nature of the mental health support experience varies widely between rainbow people. Some common threads are apparent, however: rainbow clients routinely report that mental health professionals commonly lack knowledge about rainbow needs and issues, maintain a silence around sexuality, gender, and sex characteristic diversity, and fail to challenge heteronormativity and cisnormativity in their practice.

It is impossible to ascertain the extent of negative versus positive experiences within mental health support settings, not only because of the difficulties in conducting representative studies in rainbow communities, but because questions of experience lend themselves to qualitative methods (Kuper, Lingard, \& Levinson, 2008), which do not aim to produce generalisable results. In saying this, it is likely that rainbow clients who have had negative experiences with mental health services are overrepresented in this body of literature, as they may be motivated to share their stories, while those who have had positive experiences may remain invisible (Brachman, 2011). 


\section{Has the Nature of Negative Experiences Changed Over Time?}

There is evidence to suggest that, overall, mental health professionals have moved along the 'continuum of negative experiences' over time. Indeed, research conducted in the 1990s and early 2000s (e.g. Golding, 1997; Lucksted, 2004; McFarlane, 1998; Nystrom, 1997) more commonly reported that mental health professionals were overtly discriminatory toward rainbow clients than recent research which suggests that mental health professionals discriminate in more subtle ways, such as through microaggressions, or through failing to provide adequate support due to knowledge gaps (e.g. Corliss et al., 2007; Eady et al., 2011; McCann \& Sharek, 2014; McCullough et al., 2017; Shelton \& Delgado-Romero, 2011). There are exceptions to this trend; a participant in Eady and colleagues' (2011) study reported that her provider labelled her bisexuality as “disgusting” (p. 381), while trans participants in Pitts and colleagues' (2009) study recounted instances of healthcare professionals telling them that they "needed to find god not hormones" or that they were "the filthiest most perverted thing on earth" (p. 488). In both of these studies, however, the authors did not specify a time frame in which participants had accessed mental health support. As such, it is possible that these negative experiences occurred decades before the research was published.

\section{Literature Gaps}

There are three key gaps in the literature that this study will address. Firstly, past studies on rainbow experiences of mental health support almost exclusively investigate the experiences of lesbian, gay, and bisexual people, or of trans men and women. As such, there is little understanding of the ways in which rainbow people outside of these groups experience mental health support, including, but not limited to, nonbinary, agender, bigender, queer, asexual, pansexual, genderqueer, genderfluid, and intersex people. Given that it is becoming increasingly common to identify outside of the 'LGBT umbrella' (GLAAD, 2017; Kuper, Nussbaum, \& Mustanski, 2012), it is essential and timely to conduct research on this topic that is inclusive of all rainbow identities. My research will explore the experiences of a diverse group of rainbow people, by advertising the study to people within and outside of the 'LGBT umbrella', and by focussing on recruiting previously under researched groups. 
Secondly, this study will provide much needed research on rainbow experiences of mental health support in our New Zealand context. It is essential that mental health support provision is informed by local research; experiences within New Zealand likely differ from those reported in overseas literature, due to our unique socio-political context and system for funding mental health care. On top of this, overseas research does not speak to the needs and experiences of takatāpui. A substantial body of evidence demonstrates significant mental health disparities between Māori and nonMāori (e.g. Baxter, Kingi, Tapsell, Durie, \& Mcgee 2006). No study to date, however, has explored the mental health support experiences of Māori who identify with diverse genders and sexualities.

While initial studies by Welch and colleagues (2000) and Semp (2006) were integral in beginning a conversation around queer experiences in New Zealand mental health services, their focus on lesbians and MSM limits the extent to which these studies can inform work with other non-heterosexual groups and sheds no light on the experiences of trans and intersex New Zealanders. Similarly, although Birkenhead and Rands (2012) interviewed a diverse group of mental health consumers, their analyses were not reported in a manner consistent with conventions about rigour and reliability. ${ }^{16}$ Adams and colleagues' (2012) needs assessment provided valuable insight into rainbow mental health support experiences, but its broader focus on mental health promotion and suicide prevention limited the extent to which services and support could be explored. As such, there is a dearth of New Zealand literature that specifically examines the mental health support experiences of a diverse group of rainbow community members using robust methods. Past literature also draws predominantly on the Auckland rainbow population, which, as New Zealand's biggest city, may not reflect the experiences of rainbow people in other parts of New Zealand. Moreover, no New Zealand study to date has examined the experiences of trans people who present to mental health services in order to access gender-affirming healthcare. My research will address these gaps by recruiting participants from across New Zealand, and specifically

\footnotetext{
${ }^{16}$ The method of qualitative analysis was not discussed, much of the qualitative data was presented in pie or bar graphs, and descriptive information about quantitative data was reported, but quantitative data was not analysed further.
} 
exploring the experiences of trans participants who have accessed gender-affirming healthcare.

Finally, my research will add to this body of knowledge by using both qualitative and quantitative methods, ${ }^{17}$ and by collaborating with participants to create a practical resource based on research findings for mental health professionals. Past research in this area routinely concludes with recommendations for improving the mental health care of rainbow clients (see, for example, Austin \& Goodman, 2018; Hunt et al., 2006; Lucksted, 2004; McCann \& Sharek, 2014; Simeonov, Steele, Anderson, \& Ross, 2015), but it is often unclear whether these recommendations have real-world impact beyond academic publication. On the other hand, practice guidelines for mental health professionals working with rainbow clients (for a systematic review, see McNair \& Hegarty, 2010) are often informed by research on rainbow mental health experiences, as well as authors' clinical experience, but are rarely developed in collaboration with service users. My research will, to the best of my knowledge, be the first that explores the mental health support experiences of rainbow people, uses findings to create a practice resource in consultation with participants, and shares the voices of participants through the resource.

\section{Conclusion}

There is a clear need for an in-depth study of rainbow experiences of mental health support in the New Zealand context. My research will use mixed methods to examine the experiences of a diverse group of rainbow community members who access mental health support in New Zealand. I will also explore the experience of trans people who present to mental health services in order to access gender-affirming healthcare. Local research of this kind will be invaluable for informing the work of Aotearoa's mental health professionals; I aim to use these findings to develop a resource for the provision of culturally competent mental health care with rainbow clients. In the next chapter, I reflect on my positioning in this research, and outline the theoretical

\footnotetext{
${ }^{17}$ Which, as I discuss in the next chapter, means I can draw on the strengths of both approaches; previous studies tend to use only one of these methods.
} 
framework I used as a guide my methodological decision-making, data collection, and data analysis. 


\section{Chapter 4: Positioning and Theoretical Framework}

I begin this chapter with a reflection on my positioning within the research and consider the ways in which literature on insider/outsider perspectives can shed light on the ethics of conducting 'outsider' research. I then present the theoretical framework I employed to answer my research questions. My research was a community-based project informed by queer theory. I used a mixed methods design, first completing semistructured individual and dyadic interviews with 34 rainbow community members who had accessed mental health support. I used the findings from these interviews to develop an online questionnaire, surveying a further 1575 rainbow community members who had accessed mental health support. Finally, in collaboration with participants and community advisors, I developed a resource based on my findings for use by mental health professionals in their work with queer, trans, and intersex clients.

\section{Positioning and Reflexivity}

As I alluded to in the introductory chapter of this thesis, I am not a member of the rainbow community. ${ }^{18}$ Originally, I thought this lack of lived experience meant that I approached this research from the perspective of an 'outsider'. In contrast, an 'insider' "is a researcher who personally belongs to the group to which their participants belong" (Hayfield \& Huxley, 2015, p. 91). My view of myself as an 'outsider' has shifted over the course of the project - as I explain in the coming section, I now consider myself as occupying "the space between" insider and outsider (Dwyer \& Buckle, 2009, p. 60). Nevertheless, positionality has been a near-constant source of discomfort for me throughout my PhD journey. While at times I was able to, as Acker (2001)

\footnotetext{
${ }^{18}$ Coming back to this sentence near the end of writing my thesis, it may be more accurate to say that I typically describe myself as straight, am cis, and am not intersex. Some of my rainbow friends and colleagues have (very kindly) argued that I am part of the rainbow community by virtue of my work, but I tend to phrase my positioning this way as it is easier than providing a detailed account of my own various identities.
} 
recommends, "work creatively within the tensions engendered by the [insider/outsider] debate" (p. 12), I more often questioned whether it was my place to do this work.

Issues related to insider-outsider positioning, identity, and power have been hotly contested in qualitative research (Alcoff, 1991; Bridges, 2001; Fine, 1994; Merton, 1972). Here, I provide a brief overview of the advantages and disadvantages associated with insider versus outsider positioning, before discussing work that explores the complexities of claiming an insider or outsider status, and what it means to "work the hyphen" (Fine, 1994, p.70).

\section{The Insider-Outsider Debate}

Before the mid-twentieth century, theorists in sociology, anthropology, and psychology commonly argued that conducting field research from an outsider perspective was preferable to that of an insider, as researchers outside of the group under study were able to be 'objective' in a way that insiders could not (Aguilar, 1981; Burgess, 1984; Merton, 1972; Schuetz, 1944). Those who became overly familiar with the groups they were working with were accused of "going native", or of having their objectivity "polluted" (Hellawell, 2006, p. 485). These early understandings of insider/outsider positioning were typically based on a positivist notion of reality, which assumes the existence of a single truth, independent of the knower (Merriam et al., 2001).

Researchers working within critical, feminist, and poststructuralist paradigms have challenged this notion of scientific neutrality, rejecting the assumption that there is a single reality to be observed (Britzman, 1995; Merriam et al., 2001). Rather, "the researcher's own experiences, values, and positions of privilege in various hierarchies have influenced their research interests, the way they choose to do their research, and the ways they choose to represent their research findings" (Harrison, MacGibbon, \& Morton, 2001, p. 325). As such, from a poststructuralist perspective, no researcher can be objective, regardless of insider or outsider status, as it is impossible to step outside of one's cultural context.

Although few qualitative researchers continue to assert that objectivity is attainable (or even desirable; Dwyer \& Buckle, 2009), there are, nonetheless, ongoing concerns that insider researchers may take for granted familiar aspects of their 
participants' experience, rather than viewing them as worthy of analysis (Acker, 2001; LaSala, 2003; Perry, Thurston, \& Green, 2004). Assumptions of a shared understanding between participant and researcher may also mean that participants explain their experiences less fully than they would with an outsider researcher. If insider researchers fail to seek clarification from participants during interviews, this leaves the researcher with data that potentially cannot be included in analysis (Hayfield \& Huxley, 2015). Kanuha (2000), an indigenous Hawaiian lesbian researcher who interviewed lesbians and gay men of colour in the United States, reflected on instances of this in her research:

I became aware of the many moments when I did not allow-or, more accurately, require-study respondents to complete sentences, thoughts, or descriptions because I knew implicitly what they were referring to in response to a particular line of questioning (p. 442).

In contrast, outsider researchers may act as "phenomenological strangers" who are better positioned to unpack these "taken for granted" aspects of participants' experience (Sparkes, 1994, p. 170).

While familiarity with participants' lived experience presents potential problems for the insider researcher, being a member of a group one is studying also comes with numerous advantages. Insider researchers typically gain the trust of their participants more readily than outsider researchers (Dwyer \& Buckle, 2009). Because of this, insider researchers may find the process of recruitment easier, and their participants may be more open during interviews (Grove, 2017). ${ }^{19}$ Insider researchers' understanding of the lives of their participants might also enable them to ask questions that would not occur to outsider researchers (LaSala, 2003; Merriam et al., 2001). Conversely, outsiders' lack of lived experience may limit their ability to truly understand what their participants have faced (Bridges, 2001). As a result, outsider researchers' analyses may lack the depth and breadth of those of insider researchers.

${ }^{19}$ Of course, the inverse is also possible; that participants may appreciate the opportunity to discuss their lives with a researcher who is outside of their social circle, as they are unlikely to encounter them in their day-to-day lives (Kauffman, 1994). 
The question of whether outsiders should conduct research with stigmatised or marginalised groups has been particularly controversial. One concern is that outsiders may employ pathologising frameworks of understanding in their work (Bridges, 2001). For example, as I discussed in the previous chapter, many early studies of sexuality within the field of psychology focussed on the causes of homosexuality, subscribing to a disease or disorder discourse where heterosexuality was viewed as the norm, and nonheterosexuality as illness (Kitzinger, 1999; Kong, Mahoney, \& Plummer, 2002; Tang, 2007). Although outsider researchers can circumvent this by adopting theoretical frameworks that explicitly challenge structural discrimination, their work may still disempower marginalised groups by exposing them to potential misrepresentation, or by reinforcing perceptions of dependency (James \& Platzer, 1999). hooks (1990) ${ }^{20}$ describes the ways in which privileged researchers have historically othered marginalised groups:

Often this speech about the "Other" annihilates, erases: "No need to hear your voice when I can talk about you better than you can speak about yourself. No need to hear your voice. Only tell me about your pain. I want to know your story. And then I will tell it back to you in a new way. Tell it back to you in such a way that it has become mine, my own. Re-writing you, I write myself anew. I am still author, authority. I am still the colonizer, the speaking subject, and you are now at the center of my talk" (p. 343).

As hooks points out the work of majority group members is granted an authority or legitimacy that the work of marginalised groups is not, and research by majority group members about the needs and experiences of marginalised people reaffirms this perception of an authoritative voice. Smith (1999) makes a similar argument in Decolonising Methodologies, where she notes that research is inextricably linked to colonialism and reflects on the impudence of Western researchers and intellectuals, who "can assume to know all that it is possible to know of us, on the basis of their brief encounters with some of us" (p. 30).

\footnotetext{
${ }^{20}$ bell hooks uses lowercase letters in her name "to emphasise her work rather than her identity" (Wisneski, 2013, p.73).
} 
Prior to applying for my $\mathrm{PhD}$, when I was considering mental health service provision in rainbow communities as a potential topic, ${ }^{21}$ I returned to hooks' quote frequently. I was torn between a pull to conduct research that was urgently needed, and a fear that I would become the colonising researcher that she describes. Reading Alcoff's (1991) work on the problem of speaking for others, I resonated strongly with the questions she posed:

If I don't speak for those less privileged than myself, am I abandoning my political responsibility to speak out against oppression, a responsibility incurred by the very fact of my privilege? ... Is my greatest contribution to move over and get out of the way? (p. 8, emphasis in original).

At this time, I was aware of several queer and trans academics and postgraduate students around New Zealand who were conducting important research in rainbow mental health. Given the dearth of literature in this area, however, there was a call for considerably more work to be done. I felt that placing the responsibility of conducting this research entirely on the shoulders of the rainbow community was not only unrealistic but was absolving straight and cis researchers of their responsibility to challenge queerphobia and transphobia within their fields. After seeking advice from colleagues and friends in the rainbow community, I decided to investigate ways of conducting this research that would empower and give voice to rainbow communities. In the next section, I explore reflexivity and community consultation as practices that have potential to challenge traditional power imbalances within qualitative research. I also discuss literature on working the hyphens and the space between and consider the ways in which I am both an insider and an outsider in my own research.

\section{"Working the Hyphen" and Occupying "the Space Between"}

Qualitative researchers commonly draw on Fine's (1994) notion of "working the hyphen" (p.70) to inform their choices around positionality, identity, and negotiating relationships with participants and communities (Cunliffe \& Karunanayake, 2013; Dimitriadis, 2001; Granek, 2013; Jones, 2012; Wagle \& Cantaffa, 2008). Fine (1994)

${ }^{21}$ An alternative potential topic was "cat ownership and wellbeing." It is fair to say that, had I pursued this topic, I would not have grappled with outsider issues to the same extent. 
argued that qualitative researchers work within the hyphen between Self and Other, and that "Self and Other are knottily entangled" (p. 72) in a relationship that can be unequal and, in some cases, exploitative. When we "deny the hyphen" (p. 72), writing about the Other while failing to consider our own positionality, we reproduce the colonising discourse that hooks (1990) outlines. To interrupt this, Fine (1994) suggested that qualitative researchers work the hyphen:

By working the hyphen, I mean to suggest that researchers probe how we are in relation with the contexts we study and with our informants, understanding that we are all multiple in those relations. I mean to invite researchers to see how these 'relations between' get us 'better' data, limit what we feel free to say, expand our minds and constrict our mouths, engage us in intimacy and seduce us into complicity, make us quick to interpret and hesitant to write (p. 72).

In the above extract, Fine (1994) highlighted the importance of reflexivity in analysis. Reflexivity, or "to bend back upon oneself" refers to "critical self-reflection of the ways in which researchers' social background, assumptions, positioning and behaviour impact on the research process" (Finlay \& Gough, 2003, p. ix). Unlike reflection, which takes place following an experience, reflexivity occurs before, during, and after research is conducted. Reflexivity helps to enhance understandings of the topic under investigation, and explicitly acknowledges that research findings are anchored in the social context, and are co-constituted by researchers, participants, and the relationship between research and participant (Berger, 2015).

When Fine stated that we are 'multiple' in our relations with the contexts we study and our informants, she challenges the assumption that we can mark clear boundaries between insider and outsider. Other qualitative researchers have increasingly explored the idea of multiple insider-outsider positionalities (Merriam et al., 2001). Because our identities are multidimensional and ever-shifting, Villenas (1996) claimed that researchers can be insiders and outsiders to their participants in different ways at different times. Similarly, Dwyer and Buckle (2009) argued that we must challenge the dichotomy of insider versus outsider status and posit that researchers may never fully occupy either one or other of these positions. Rather, researchers may only ever occupy "the space between" (p. 60), or that of an insider-outsider. 
When I reflected on my own "subtly varying shades of 'insiderism' and 'outsiderism'” (Hellawell, 2006, p. 489) I realised that sex, gender, and sexual orientation were not the only salient aspects of my identity when I met with participants - in fact, we had more in common than I had originally considered. At the time of conducting the interviews I was 23 - exactly in the middle of my 16-30 age bracket. I had lived in both Auckland and Wellington, where most of my interviews took place, and was a student. My interview participants and I built rapport through conversation about where we had lived, worked, and studied. We also discussed mutual friends and acquaintances, or organisations we had been involved in.

Perhaps the most powerful point of connection I shared with participants related to our perspectives on political and social issues. Most, like me, identified as intersectional feminists, were left-wing/liberal voters, and viewed sexuality and gender as socially constructed, rather than essential or fixed. ${ }^{22}$ Overall, I found that these points of commonality made it relatively easy to build relationships with participants, despite my identities as straight and cis, and as a clinical psychologist in training. ${ }^{23}$ Throughout the research process, I kept a reflective journal, where I documented my thoughts about my relationships with participants, and about the ways in which my subject positions impacted the research at each stage.

Although no researcher can be fully described as an 'insider' or 'outsider', and while self-reflexive analysis can challenge conventional scientific ideas of distance and objectivity, it is important to note that reflexivity alone may not be enough to challenge unequal power imbalances in qualitative research. As such, self-reflexive analysis can be paired with methods that disrupt exploitative relationships between researchers and participants, such as community-based research or co-operative inquiry (Israel, Schulz,

\footnotetext{
${ }^{22}$ By 'socially constructed', I mean that sexuality and gender are not predetermined at birth, but that their meanings are socially determined, and vary across time and place. I discuss this in further detail in the 'theoretical framework' section of this chapter.

${ }^{23}$ I originally wondered whether my position as a training mental health professional would excerabate the existing researcher-researched power dynamic, as it might reflect the the power imbalance in therapist-client relationships. During interviews, I did not feel that this was the case, perhaps because I was so early on in my training, or perhaps because participants understood me as part of a new generation of mental health professionals eager to create safe and inclusive therapeutic spaces for rainbow people. Of course, this is only my perspective - in hindsight, I would have loved to ask participants how my subject positions impacted on their interview experience.
} 
Parker, \& Becker, 1998; Macaulay et al., 1999; Minkler, 2005). These collaborative approaches, consistent with kaupapa Māori research methodology (Smith, 2015), highlight the importance of working with, rather than on people (Heron \& Reason, 2001). Community-based research treats communities as active agents, rather than passive subjects, and ensures that research is only conducted when it is of direct benefit to communities (Edwards, Lund, Mitchell, \& Andersson, 2008). I discuss these collaborative approaches in further detail in the theoretical framework section of this chapter and describe the process of consulting with community members in my own research.

\section{Theoretical Framework}

Grant and Osanloo (2014) liken the selection and integration of a theoretical framework in dissertation research to creating the blueprint for one's house; just as a blueprint serves as a guide for building all aspects of the home, "the theoretical framework is the foundation from which all knowledge is constructed (metaphorically and literally) for a research study" (p. 12). Failing to identify the theoretical framework leaves a research study without a structure or organising force - much like a house without a blueprint. Moreover, the choice of theoretical framework is not arbitrary, but reflects the researcher's values, as well as their beliefs about the nature of knowledge (Lysaght, 2011). In this section, I discuss how queer theory, mixed methods, and communitybased research work together to form the theoretical framework for my research. I provide a visual representation of this theoretical framework in Figure 2.

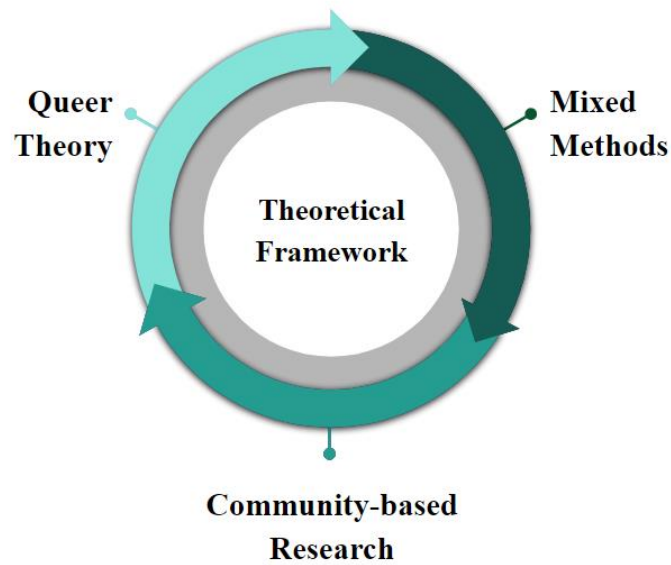

Figure 2. Theoretical framework for the current study. 


\section{Queer Theory}

Queer theory is a diverse field that originated from the work of scholars such as Michel Foucault, Judith Butler, and Eve Kosofsky Sedgwick (Jagose, 1996). While there is no one definition of queer theory, queer theorists generally draw on poststructuralist understandings of knowledge and reality. Put simply, poststructuralism assumes that all aspects of human experience are textual; that all that we know of ourselves and our world is based on language (Gavey, 1989). Poststructuralists reject notions of universal truth and singular reality and understand knowledge as partial and contextual. As mentioned earlier in this chapter, poststructuralists emphasise that the background, positioning, and values of a researcher influence the knowledge they produce (Acker, 2001).

Queer theorists apply poststructuralist ideas to sexuality, gender, and sex characteristic diversity, and challenge taken-for-granted assumptions about identity. Queer theorists also attempt to move beyond gay and lesbian studies to acknowledge other sexualities, and to take a more critical approach to gender and sexuality (Halperin, 2003). From this perspective, identity politics - social or political movements based on shared marginalised identities - harm, rather than help, rainbow communities, as categorical understandings of sex, gender, and sexuality work to maintain unequal power relations between groups (Gamson, 1995). In saying this, many queer theorists recognise the impossibilities of working outside identity; even as we reject identity, we are constituted in relation to it. Butler (1991) notes that identity categories can serve pragmatic purposes, writing that "this is not to say I will not appear at political occasions under the sign of lesbian, but that I would like to have it permanently unclear what precisely this sign signifies" (p. 14).

Rather than understanding gender and sexuality as fixed, immutable, and binary, queer theorists view sexuality and gender as potentially fluid and nonbinary (Jagose, 1996). Gender is something we 'do' (and that does us), rather than something we 'are'. Because there is no one truth about who we 'are' as people, we can occupy or take up multiple identities in different contexts and at different times (though it is important to note that the identities we occupy or take up are constrained by social norms, and by the consequences of troubling these norms). As well as destabilising identity categories, queer theory destabilises categories of 'normal' and 'natural', challenging 
heteronormative and cisnormative frameworks (Halperin, 2003). Sedgwick (1993) defines queer as "the open mesh of possibilities, gaps, overlaps, dissonances and resonances, lapses and excesses of meaning when the constituent elements of anyone's gender, of anyone's sexuality aren't made (or can't be made) to signify monolithically" (p. 8, emphasis in original).

Although queer theory is an approach primarily focussed on sexuality, gender, and sex characteristic diversity, theorists are increasingly incorporating intersectional approaches in queer analyses (Fotopoulou, 2012). In the context of this thesis, and consistent with my previous discussion regarding positionality, participants' experiences of mental health support are influenced by not only their sexuality, gender, and sex characteristics, but also by their other identities and subject positions. A failure to acknowledge this could, as Chevrette (2013) argues, mask other social divisions that intersect with rainbow identities, and could also reaffirm notions that there is a singular experience of queerphobia, transphobia, or intersex discrimination.

Queer theory is a powerful tool for deconstructing normative understandings of sexuality, gender, and sex characteristic diversity within research. I adopt this theory as a framework from which to understand participants' experiences not only because they align with my own beliefs regarding sexuality, gender, and sex characteristic diversity, but because I have an ethical obligation to work from a rainbow-affirmative perspective. As I have previously discussed, researchers and clinicians have historically understood marginalised identities as disordered and have searched for the causes of rainbow identities. This has had significant negative consequences for the health and wellbeing of queer, trans, and intersex people (for example, through conversion therapy, human rights abuses, discrimination, and social exclusion). As such, it is essential that I take an explicitly non-pathologising perspective, so as to avoid perpetuating harm to the rainbow community.

\section{Mixed Methods}

Mixed methods, an approach in which qualitative and quantitative methods are systematically integrated within a single project, is increasingly recognised as the "third research paradigm" (Johnson \& Onwuegbuzie, 2004, p. 13). The goal of mixed methods is to draw on the strengths (and minimise the weaknesses) of both qualitative and 
quantitative approaches (Creswell \& Plano Clark, 2007). In quantitative research, for example, the voices of participants are rarely heard, and researchers often fail to acknowledge the impact that their identities and subject positions have on the research process and findings (Creswell \& Plano Clark, 2007). In contrast, qualitative research is critiqued for its lack of generalisability, and for the 'bias' created by personal interpretations on the part of the researcher (Carr, 1994).

As outlined above, my research is informed by queer theory, which is in turn informed by poststructuralist theory. As such, I see truths as multiple and contextbound; I am unconcerned about the personal interpretation and lack of generalisability that comes with qualitative research done well and see quantitative findings as one possible story about a particular phenomenon, rather than as evidence of an 'ultimate truth'. Because of this, I employ mixed methods not to overcome the oft-cited 'weaknesses' of qualitative research (though this is a happy by-product) - my reasons for using mixed methods are entirely pragmatic (see Feilzer, 2009). Quantitative studies are consistently placed higher on 'evidence pyramids' than qualitative studies (Levin, 2014). These evidence pyramids, in turn, are used by government and community organisations in their decision-making processes. As such, I use mixed methods in an effort to ensure my findings and resource will be taken seriously and used to drive change within government ministries, DHBs, non-governmental mental health organisations, and university training programmes.

In this study, I use a sequential transformative design. This has two distinct data collection phases, conducted one after the other (either method may be used first; Creswell, Plano Clark, Gutmann, \& Hanson, 2003). For the purposes of this project, in the absence of an extensive local research literature from which to draw, I first conducted a qualitative study of the experiences of rainbow people who accessed mental health support in New Zealand. I used the findings from this study to develop a (predominantly quantitative) survey about the experiences of rainbow people who accessed mental health support in New Zealand and integrated the findings from these studies to develop a resource for use by mental health professionals in their work with rainbow clients. As Hanson, Creswell, Plano Clark, Petska, and Creswell (2005) note, the sequential transformative design is useful for giving voice to diverse perspectives and advocating for research participants. 


\section{Community-Based Research}

Community-based research is a collaborative approach in which research takes place in, and is of benefit to, the community under study (Israel et al., 1998). Community members are involved in the design and implementation of every aspect of the research process, enhancing the relevance and utility of the research data. Consistent with a reflexive approach, the goal of community-based research is to work with, rather than on people, in order to create social change and achieve social justice (Heron \& Reason, 2001; Minkler, 2005). As mentioned previously, community-based research has the potential to disrupt unequal power relations between the researcher and researched, and to ensure that communities are not exploited in the course of participating in research (Wallerstein \& Duran, 2006).

Literature on community-based research often fails to differentiate between community-placed research, community-based research, and community-based participatory research. 'Community-placed research' refers to research that takes place within a community setting, but is researcher-led, rather than community-partnered (Horowitz, Robinson, \& Seifer, 2009). For example, researchers may conduct a study at a local school, but without consulting teachers or school staff in the development of the research questions or study design. In Jacquez, Vaughn, and Wagner's (2013) recent review of community-based participatory research with youth, one third of the 104 community-placed studies identified in the review had been described as 'communitybased participatory research' by the study authors. This suggests that researchers commonly misrepresent their community-placed research studies as community-based participatory research.

Distinguishing 'community-based research' from 'community-based participatory research' is more difficult than differentiating between these approaches and community-placed research, as the extent of collaboration between researchers and communities varies dramatically between studies. Clarifying these differences, however, is critical, given that the approach used within research has implications for community empowerment and study validity (Jacquez et al., 2013). While community members participate, to some degree, in the process of community-based research, community-based participatory research takes this one step further: Community members and researchers are equal partners in the research process, jointly collecting 
and reviewing data, sharing control of decision making, and sharing funding received for the research (Bergold \& Thomas, 2012). For example, researchers leading a community-based study may seek advice from community members at each stage of the research process and disseminate findings to the community in an accessible format at the conclusion of the study. In a community-based participatory study, however, researchers and community members form a team in which researchers may train community members to collect data that the researchers and community members then analyse together (Jacquez et al., 2013).

Community-based participatory research is typically more time consuming than other research approaches, as researchers must build genuine, enduring relationships with community members before the research process begins. In Castleden, Morgan and Lamb's (2012) study of researchers' perspectives on their community-based participatory research, one participant noted that they spent the first year of their study drinking tea, rather than collecting data, as this was the time it took to build trust in the community they were working with. Community-based participatory research also entails a significant time commitment on the part of communities, which can be burdensome for those that are already overworked and under resourced (Israel et al., 2006).

Although I began the project determined to conduct research using a collaborative approach, I was open to conducting a community-based research study or a community-based participatory research study. The decision to conduct communitybased research, rather than community-based participatory research, was made following a series of meetings with leaders in New Zealand's rainbow community. As I discuss in the next section, rainbow community members were in full support of the project and wanted to be involved throughout the research process. It was not feasible, however, for community members to jointly conduct the research, due to their own time constraints, and my resource constraints. As such, although community members were involved at each stage of the research process, it would be disingenuous to say we were equal partners; I conducted all interviews, analysed the data, and was the final decision maker on matters related to the research. 


\section{Summary}

Before I began collecting data to answer my research questions I considered my positioning within this research, how I could conduct ethical and helpful research as someone outside of the rainbow community, and which theories and methods would form a foundation from which to guide the research process. While I still consider myself an 'outsider' in the rainbow community, I now understand myself as "occupying the space between" insider and outsider in this research (Dwyer \& Buckle, 2009, p. 60). In relation to participants in the current study, I am both an insider and outsider in different ways and at different times. I take a reflexive approach to my work in order to challenge conventional scientific ideas of distance and objectivity, and to stay accountable to participants and their wider communities. My theoretical framework of queer theory, community-based research, and mixed methods is consistent with my understanding of insider/outsider positioning and fits with my values as a researcher and beliefs about the nature of knowledge. In the next chapter I present an overview of my interview methodology, to demonstrate the ways in which the theoretical ideas presented in this chapter influenced my decision-making during the research process. 


\section{Chapter 5: Interview Methodology}

In this chapter I describe the process of conducting interviews with 34 rainbow young adults. I first provide an overview of my community consultation, recruitment, and participant demographic information. I then outline my method of data collection and analysis.

\section{Community Consultation}

I began my project by contacting rainbow community groups and organisations in Wellington and Auckland. I spoke with them about my background (both personal and professional), described my proposed research, and asked each person what they saw as the key issues facing their community, particularly when it came to accessing mental health support. I then asked if they would like to collaborate with me throughout the research project in whatever form they saw fit. Community organisations volunteered to contribute to the research to different extents, based on their own resources and interest in the project. In total, more than 15 community organisations and leaders were involved in some way over the course of the project. Three organisations (InsideOUT, Gender Minorities Aotearoa, and RainbowYOUTH) volunteered to be research partners, and went on to form a core group of advisors for all major project decisions.

Community leaders advised that they needed research that would increase the cultural competence of mental health professionals in their work with rainbow clients and demonstrate the positive effects of rainbow-affirmative healthcare on mental health and wellbeing. We began to discuss the idea of developing a practical guide for use by mental health professionals. In hui with community consultants, we also discussed what data we should collect to develop the resource from. We decided to begin with interviews with queer, trans, and intersex clients, as any resource guiding work with rainbow clients should be informed by their experiences.

Because the eligibility criteria was so broad in regards to sexuality, gender, and sex characteristic diversity, I decided to narrow my age range to interview people 
between ages 16 and 30. This ensured that participants' experiences of accessing mental health support would be, for the most part, restricted to within the last 10-15 years. ${ }^{24}$ Although I considered interviewing rainbow community members under the age of 16, Victoria University of Wellington's Human Ethics Guidelines state that children under the age of 16 must obtain parental consent to participate in any study (Victoria University Research Policy Group, 2018; but see pages 155-156 for further discussion). Because many rainbow New Zealanders under the age of 16 have not yet come out (Lucassen et al., 2014), I was concerned that this may pressure young people into coming out to their parents in order to participate.

\section{Recruitment}

This project was approved by the Victoria University of Wellington Human Ethics Committee (RM\# 0000024394; see Appendix A). ${ }^{25}$ Participants were recruited using a flyer distributed online (see Figure 3). In an effort to capture the attention of potential participants, I used colours from the rainbow flag and the trans flag. ${ }^{26}$ I also included several different identity descriptors (queer, takatāpui, nonbinary, trans, LGBTQIA+, MVPFAFF, and questioning) to make clear that all rainbow community members were eligible (provided that they met the other two criteria), regardless of the specific term they used to describe their identity.

\footnotetext{
${ }^{24}$ While the aim of the study is not to produce generalisable results, it is arguably appropriate to base a resource on the most recent experiences possible, especially given that, as discussed in the previous chapter, the nature of experiences appears to have shifted over time.

25 The process of ethical decision-making extended far beyond my application for University ethical approval. Indeed, Miller and Boulton (2007) suggest that there is a growing mismatch between the standardised procedures of research ethics committees and "complex social worlds and research encounters which do not fit neatly into boxes which can be ticked" (p. 2199). See Appendix B for a discussion of ethical considerations related to the principles of autonomy and beneficence.

${ }^{26}$ This included a rainbow-coloured version of the Victoria University of Wellington logo that may have violated institutional policy, however a cursory search of the Research Policy, Information Security Policy, Intellectual Property Policy and Naming Right Statute found no evidence for any violation.
} 


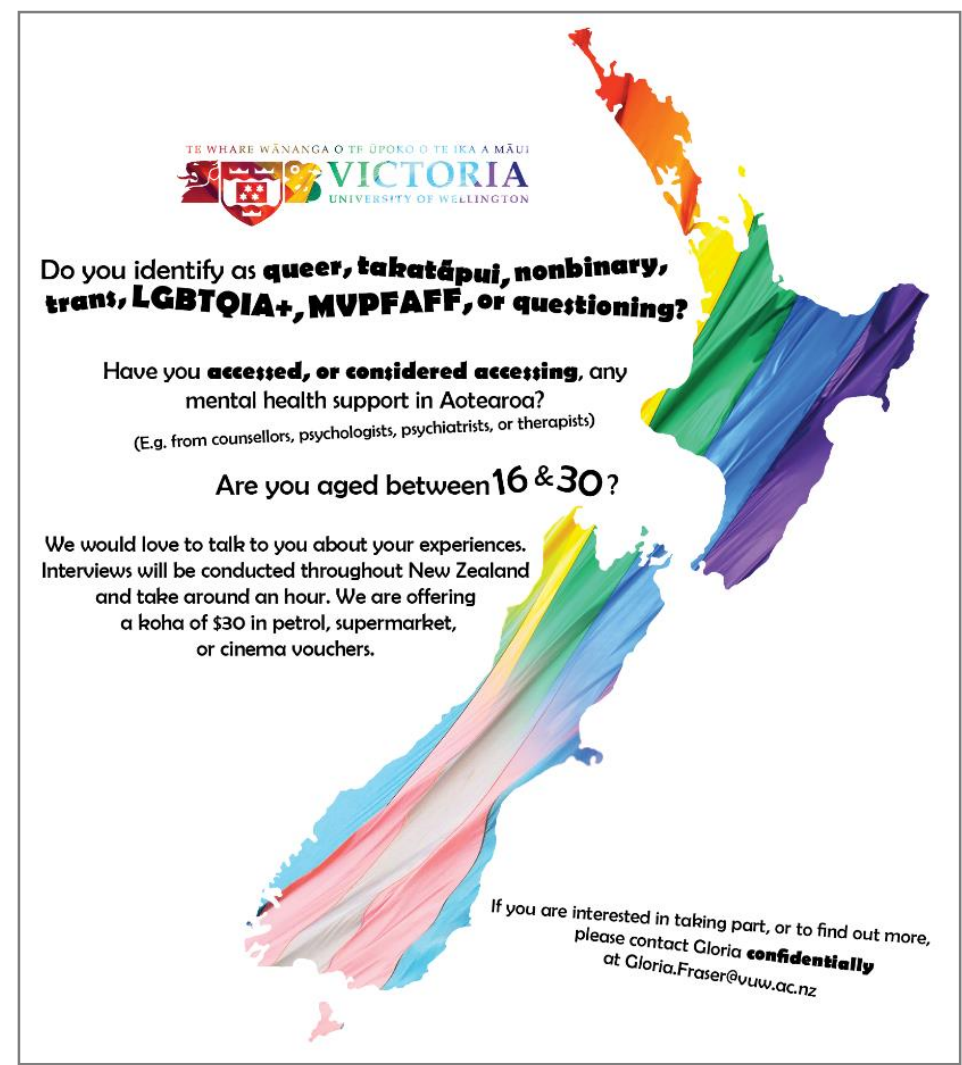

Figure 3. Interview study recruitment flyer

Design of the recruitment flyer went through a number of iterations as community advisors' feedback was incorporated. After the final flyer design was approved by community advisors and I had obtained ethical approval for the study, I emailed a copy of the flyer to all of the community organisations I had met with to discuss the project, so that they could distribute the flyer via email, their website, and on social media. I also circulated the flyer to my friends and colleagues who are either part of, or have connections to, the rainbow community, and posted the flyer to my personal Facebook page, making the post publicly available.

In total, I was contacted by 109 potential participants: 17 through Facebook and 92 via email. The majority of these participants contacted me within the first week of recruitment. I responded to the first 15 people who expressed interest, then, realising I was going to receive expressions of interest from more people than I could interview, met with my supervisors to form a plan before responding to any further messages. In collaboration with my supervisors, I decided to prioritise interviewing groups who had historically been underrepresented in this area of research, including intersex people, 
trans people (specifically nonbinary trans people), and takatāpui (Treharne, 2011). My aim was not to interview a group that was representative of the rainbow community, but to ensure that a diverse range of people were included with regard to sexuality, gender, sex characteristics, ethnicity, and location in New Zealand.

In their responses to the flyer, many potential participants had included information about identities as queer, trans, and/or intersex. I created a spreadsheet of all the information I had about potential participants, and responded to all self-identified intersex, takatāpui, and trans people, as well as those who advised they lived in the Wellington region $(n=22)$. I sent all other respondents $(n=72)$ an email explaining my situation; that I had received expressions of interest from more people than I could interview and was unable to interview them at the present time. I asked if I could hold onto their details and contact them in case someone else in the area cancelled their interview, or if I conducted other studies later in my $\mathrm{PhD}$. Of the 72 people that received this email, 43 agreed to this. The remaining 29 did not respond.

I sent potential participants an email explaining that I was starting with interviews in Wellington and Auckland and was intending to travel elsewhere in New Zealand later in the year. ${ }^{27} \mathrm{I}$ attached an information sheet about the study (see Appendix C), and the interview guide (see Appendix D) to the email. I advised that the interview guide was attached for their interest, and that there was no need to prepare for the interview in any way. I also let potential participants know that if they wanted to find out more about me or my research before deciding whether or not to take part, I would be happy to chat with them via email, over the phone, or in person. ${ }^{28}$ If potential participants did not respond to this email, I sent a follow-up email one week later to confirm they were still interested in the study. If they did not respond to this second email, I emailed the next potential participant on the list, inviting them to participate. If potential participants were still interested in taking part after reading the information

\footnotetext{
${ }^{27}$ At the time recruitment began, I had confirmed that I would be conducting interviews in Wellington and Auckland but was awaiting the results of grant applications to determine whether I could travel elsewhere in New Zealand to conduct interviews.

${ }^{28}$ No one took up the offer to talk in person or on the phone, but some potential participants asked questions via email before agreeing to take part.
} 
sheet and interview guide, I organised an interview with them at a time and location of their choice.

Within qualitative research there are no hard and fast rules for how many interviews are needed for an analysis. Qualitative researchers have commonly been guided by the concept of saturation, meaning that they continue to collect data until doing so sheds no further light on the phenomenon under study (Fusch \& Ness, 2015). The appropriateness of using saturation to justify the point at which interviewing stops has been widely critiqued; as Low (2019) highlights, the concept of saturation "is a logical fallacy, as there are always new theoretical insights to be made as long as data continues to be collected and analysed” (p. 131). Braun and Clarke (2019a) advocate for a pragmatically determined sample size instead, which involves "guestimating a provisional, anticipated lower and/or upper sample size or range that will potentially generate adequate data to tell a rich, complex and multi-faceted story about patternings related to the phenomena of interest" (p. 11). This provisional sample size is generated for the purposes of research proposals and ethics and funding applications. Braun and Clarke (2019a) then suggest that researchers make a decision during data collection regarding the point at which interviewing should end. The final sample size is shaped by the amount of data needed to adequately address the research question, as well as by practical constraints such as the time and resources available.

Using Braun and Clarke's (2019a) guidelines I initially guesstimated I would interview 20 people, based on past studies in this area of research and the time and resources I had available. Upon receiving so many responses to my flyer, my supervisors and I decided that I would increase this number to 30. I ended up with a final sample size of 34 , as it was at this point that I decided I had collected the data needed to meaningfully address my research questions.

\section{Participants}

In total, I interviewed a total of 34 rainbow young adults about their experiences of accessing mental health support. In order to contextualise participants' experiences during analysis, I collected relevant demographic data from each participant; their selfreported age, gender, sexual orientation, and ethnicity. I explained that providing this information was optional, and that it would be used to provide background information 
about the overall sample, as well as to provide context for any excerpts from their interview.

Here I present participants' demographic information in the aggregate, to ensure that participants are not inadvertently identifiable. For the same reason, I do not report the iwi of takatāpui participants. While it is important to acknowledge diversity within Māori communities (Forster, 2003), I see participant confidentiality as an ethical priority in this case.

At the time of data collection, participants described their gender as follows: four as cis men; one as a questioning cis man; nine as cis women; five as trans men; four as trans women; one as trans femme; one as an intersex female; three as nonbinary; one as a nonbinary trans woman; one as nonbinary/genderqueer; one as agender; one as a trans goth; one as transfluid; one as genderqueer, transfeminine. With regard to pronouns, 10 participants used he/him pronouns, 16 used she/her pronouns, and six used they/them pronouns. One participant used both he/him and they/them pronouns, and one participant used both she/her and they/them pronouns, and requested I alternate between these in my analysis. Participants' correct pronouns are used at all times throughout this thesis.

Participants described their sexual orientation as follow: 12 as queer; five as gay; three as lesbian; four as bisexual; five as pansexual; one as takatāpui; one as pansexual, polyamorous; one as asexual, hyperromantic; one as takatāpui, queer; one as straight.

Participants' ages ranged from 17 to 30 years $(M=23$ years $)$. Eight participants were aged between 17 and 20, nine participants between 20 and 23, eleven participants between 24 and 26, and six participants between 27 and 30. The majority of participants (24) identified as Pākehā. Four participants identified as Māori and Pākehā, one as Māori, two as European, two as British, and one as Pākehā/Canadian. Seven participants were born overseas, and three had accessed mental health support both within and outside of New Zealand. In these cases, I did not include data pertaining to mental health support accessed overseas in my thematic analysis, but used this data to inform resource development, as this data often contained descriptions of what participants had found helpful and unhelpful when accessing support. Participants were 
invited to select their own pseudonyms for use in this thesis and related publications. For those who did not want to select their own, I chose a pseudonym.

\section{Data Collection}

Participants were given the option of taking part in an individual semi-structured interview or taking part in a focus group. I chose to combine these methods so that participants could select the context in which they felt most comfortable; some participants may feel inhibited in a group situation, while for other participants, the presence of others may alleviate some pressure, as they would not need to respond to every question. I gave participants the option of bringing a support person to the interview, though no participants chose to do so.

Of the 34 people I interviewed, 28 chose to take part in an individual interview and six chose to take part in a two-person focus group (two of these pairs were romantic partners, and the other a pair of close friends). I later reconceptualised these "focus groups" as dyadic interviews (Morgan, Ataie, Carder, \& Hoffman, 2013). Dyadic interviews are more similar to focus groups than individual interviews, as comments from one participant can bring forth responses from the other. As Morgan and colleagues (2013) point out, however, dyadic interviews are more than just "miniature focus groups" (p. 277); unlike focus groups, dyadic interviews closely reflect an ordinary conversation between two partners (the only difference being the presence of an interviewer asking questions and facilitating conversation). Moreover, dyadic interviews produce more data from each participant than focus groups, allowing each participant to share their personal narrative in relation to the research topic.

The interviews were carried out between May and September 2017. Interviews took place at a University campus, university premises, participants' homes, or a bookable room in a community space (e.g. central city library or community organisation). Discussions ranged from 48 minutes to 124 minutes in length, with an average length of 76 minutes. Where possible, I began by offering participants a cup of tea or coffee and had biscuits available during the interview. I briefly introduced myself, gave participants an opportunity to read over the information sheet again, and asked if they had any questions about me or the interview. Prior to beginning the interview, participants filled out a consent form (see Appendix E). Following the interview, 
participants were given their choice of a $\$ 30$ Countdown voucher, a $\$ 30$ MTA voucher, or two Lighthouse cinema vouchers (valued at \$35) as a koha to thank them for taking part. No participants chose to withdraw participation, either during or following their interview.

I carried out conversations with participants using a semi-structured interview guide. This guide was designed to ensure consistency across all interviews, while allowing flexibility during the conversation. The interview guide was predominantly based on Birkenhead and Rands' (2012) interview guide for rainbow service users past and present experiences within New Zealand's mental health and addictions services. I adapted these questions and added extra questions based on a review of previous literature, as well as the recommendations of rainbow community advisors and academics who had previously completed research in the area of rainbow mental health.

The interview guide was separated into seven sections: (1) brief introductions of myself, the project, and participants; (2) engagement with mental health support; (3) discussing sex, sexuality, and gender in mental health support services; (4) satisfaction with mental health support and gender-affirming healthcare services; (5) improvements to mental health services; (6) feedback about the project; and (7) closing. Before my first interview, I completed a practice interview with a classmate using the interview guide and made minor changes to wording and flow according to their feedback. Each conversation was recorded on two audio devices, in case one device failed. Two of the dyadic interviews were also video recorded, with permission of participants, for ease of transcription. I transcribed all interviews myself. This was partially to maintain participants' confidentiality, and partially due to resource constraints at the time of data collection.

I transcribed the interviews into NVivo11 software, a programme for organising and analysing qualitative data. Interviews were transcribed verbatim, and included indications of hesitations (e.g. um, uh, mmhmm), laughter, and repetitions. In cases where I could not discern what the participant had said, I noted this with (inaudible). After transcription was complete, I reviewed the transcripts and removed all potentially identifying information, including names of people, places, and services, as well as participants' personal details. I replaced these with a short description in square brackets, such as [university], [youth health service], [hometown], or [current location]. 
The consent form indicated that all participants were given the opportunity to read and/or edit their transcript. There is some debate in the literature as to whether qualitative researchers should send interview transcripts back to participants. Forbat and Henderson (2005) note that the decision to share transcripts is often driven by a desire to empower the participant, but that participants may feel threatened by the process, particularly if they are embarrassed about what they have said, or feel their speech is inarticulate. While cognizant of this, I offered for participants to review their transcripts as this was consistent with my collaborative approach, and reassured participants that there was no pressure to articulate themselves exactly as they wanted to during the interview, as they would have an opportunity to revise their words if needed.

I emailed the de-identified transcript to those participants who had requested a copy and invited them to edit it freely. This included adding or removing any content they wished. I asked them to send the transcript back within one month, if they intended to edit it. Dyadic interview participants were asked to edit only their words, and to keep the transcript confidential unless the other person in the dyadic interview gave permission to share it. For participants that had not requested a copy of their transcript, I emailed to advise them that the transcript had been typed, and that they were still free to read or edit it if they wanted to. In response to my emails, eight participants advised they did not wish to edit their transcript, 13 reviewed their transcript and made no changes, nine edited their transcript, and four did not respond regarding their transcript. When participants edited their transcripts with tracked changes, I accepted all changes, and used this version of the transcript as the final version.

\section{Data Analysis}

Data were analysed using thematic analysis, a method for identifying, analysing, and reporting patterns of meaning (themes) within a data corpus (Braun \& Clarke, 2006). A theme "captures something important about the data in relation to the research question" (p. 10). Braun, Clarke, and other leading proponents of thematic analysis differentiate between three broad approaches to thematic analysis: a coding reliability approach, a codebook approach, and a reflexive approach (Braun, Clarke, Hayfield, \& Terry, 2018). I adopted a reflexive approach, which emphasises "meaning as contextual or situated, reality or realities as multiple, and researcher subjectivity as not just valid 
but a resource" (p. 848). I followed Braun and Clarke's (2006) guidelines for conducting a thematic analysis, which includes six phases: (1) familiarising yourself with your data; ${ }^{29}$ (2) generating initial codes; (3) searching for themes; (4) reviewing themes; (5) defining and naming themes; and (6) producing the report.

Braun and Clarke (2006) describe thematic analysis as a flexible method which can be used across a range of theoretical approaches and provides a rich and detailed account of the data. Furthermore, they argue that thematic analysis is not a passive process; themes do not 'emerge' in the process of analysis. Rather, the researcher takes an active role in identifying themes. The themes that are selected and reported reflect the position and theoretical framework of the researcher (Braun \& Clarke, 2006). I took a critical realist approach to my analysis, which "assumes an ultimate reality, but claims that the way reality is experienced and interpreted is shaped by culture, language and political interests" (Braun \& Clarke, 2013, p. 329). I chose critical realism for its ability to acknowledge the materiality of participants' experiences; constructionism, in contrast, has been critiqued for a focus on discursive practices alone (Ussher, 2010). As discussed earlier in this chapter, my thematic analysis was also informed by queer theory, meaning that as I conducted my analysis I considered the ways in which normative understandings of sexuality, gender, and sex characteristic diversity may have shaped participants' therapy experiences.

Once all participants had the opportunity to review their transcript, I read and reread all transcripts before beginning the coding process. During this familiarisation stage, I took brief notes about aspects of the data that I found particularly important or interesting. I integrated these notes with those I had taken while transcribing. The next stage in data analysis was the development of codes. Braun and Clarke (2013) describe coding as "the process of identifying aspects of the data that relate to your research question" (p. 206). I used complete coding, rather than selective coding, meaning I coded anything within my dataset that was of relevance to my research questions. Codes can also be at the semantic or latent level, where semantic codes provide a summary of

\footnotetext{
${ }^{29}$ For me, familiarising myself with the data began with transcription, and I took notes during the transcription process about aspects of the data that stood out to me.
} 
the explicit content of the data, and latent codes identify implicit meanings within the data (Braun \& Clarke, 2013). I used a combination of semantic and latent codes.

During the coding process, I hired two researchers from the rainbow community (Sam Duffy and Franziska Pöschl ${ }^{30}$ ) to form a coding team. At the time of coding, Franziska was a counselling student and provisional member of the New Zealand Association of Counsellors, working in youth mental health. She identifies as a Pākehā/German bisexual cis woman. Sam is a graduate from Victoria University of Wellington with an honours degree in Psychology. He identifies as a Pākehā bisexual cis man. At the time of coding, Sam was working as a researcher on a number of social justice and equity-related research projects.

Braun and Clarke (2006) do not recommend the use of multiple coders to achieve inter-rater reliability in thematic analysis, as this is based on realist assumptions that there is a single 'reality' in the data to be captured through coding. They note that "coding (is) an active and reflexive process that inevitably and inescapably bears the mark of the researcher(s). With no one 'accurate' way to code data, the logic behind inter-rater reliability (and multi-independent coders) disappears" (Braun \& Clarke, 2019b, p.5). In hiring researchers to form a coding team, I was not aiming for agreement in coding between myself and the two researchers. In fact, I expected that we would all code the dataset quite differently, as a result of our varied backgrounds and positioning. I invited Sam and Franziska to analyse the data with me as community-based research, as discussed earlier in this chapter, involves a partnership approach to research, where the group under study is engaged collaboratively in the research process. To involve community members in the development of the research question, recruitment, and data collection, only to conduct the analysis singlehandedly, seemed contrary to the principles of community-based research.

Before providing Sam and Franziska with interview transcripts, I emailed all participants with Sam and Franziska's names, and asked if the participant knew, or had ever met them. I provided Sam and Franziska with 13 transcripts each from participants who had confirmed that they did not know them. They both coded four transcripts and

\footnotetext{
${ }^{30}$ Sam and Franziska both agreed to be named in this thesis and any associated publications.
} 
read over a further nine transcripts for context of the overall dataset. I then met with both Sam and Franziska twice individually to discuss our coding, as well as aspects of the data that we found particularly interesting or important. Finally, my supervisors and I met with Sam and Franziska together to discuss the project as a whole, and our initial impressions of potential themes.

After data had been coded and collated, I began the process of sorting codes into potential themes. Here I used the transcripts I had coded, and occasionally referred back to Sam and Franziska's coded transcripts and notes. I collated codes for potential themes into 'theme piles' so I could read all extracts for a potential theme and check whether the data cohered together meaningfully. I also created a series of thematic maps, to examine the relationships between themes and sub-themes. As part of this process I identified the first set of four candidate themes. These candidate themes covered participants' talk about access to gender-affirming healthcare, barriers to accessing mental health support, coming out in therapy, and the therapy experience itself.

On review, data in three of these themes were too diverse. I retained the Barriers to Accessing Mental Health Support theme (renaming it "Accessing Healthcare is like a Game of Strategy” using a participant's quote) and reworked Coming Out in Therapy and The Therapy Experience into four new themes: Therapy as a Microcosm, Signalling Safety, Lack of Knowledge, and Clinical Skills. I added one more theme, Rainbow Champions. I had difficulty incorporating the remaining theme-Access to Genderaffirming Healthcare - into the analysis. Although there were some areas of overlap, participants' experiences during gender-affirming healthcare assessment seemed so distinct from those in therapy that I decided it would be appropriate to conduct a separate analysis of this data. This theme became three themes within my second analysis: Lack of Funding and the "Postcode Lottery", Proving Gender, and The Trans Narrative. The two thematic analyses, together with their final thematic maps, are presented in Chapters 6 and 7.

\section{Summary}

In preparation for conducting interviews I engaged in several months of rainbow community consultation and recruited participants through a multitude of channels. In 
total, I interviewed 34 rainbow young adults and carried out two thematic analyses of interview data: one focussed on participants' experiences of accessing mental health support, and another on trans participants' experiences of accessing gender-affirming healthcare. In the next two chapters, I present my thematic analyses of interview data and consider how these findings fit within the wider research literature. 


\section{Chapter 6: Analysis of Interviews About Accessing Mental Health Support}

The previous chapter outlined the methodological decisions I made when interviewing 34 rainbow young adults about accessing mental health support. In this chapter I discuss six key themes I identified in analyses of these accounts, in order to consider how the provision of mental health care can be improved for New Zealand's rainbow people.

The first theme, "Accessing Healthcare is like a Game of Strategy"," 31 examines the structural barriers participants described in the process of accessing mental health support. Theme two, Therapy as a Microcosm, explores the societal stigma and discrimination that participants experience in their daily lives, and the extent to which they see mental health settings as reflective of our hetero/cisnormative society. This is followed by three themes that focus on participants' therapy experiences. Signalling Safety refers to participants' thoughts as to whether questions about rainbow identities should be included in mental health assessments, as well as their suggestions for how mental health professionals can create safe spaces for disclosures around rainbow identity. Lack of Knowledge discusses participants' experiences of educating mental health professionals, who they see as often lacking knowledge about sexuality, gender, and sex characteristic diversity. Clinical Skills encompasses participants' talk about the importance of basic clinical skills in therapy, such as affirmation, validation, and checking clients' understandings of their difficulties. Finally, Rainbow Champions explores the care that rainbow people have for their communities, and the resilience they demonstrate in the face of hardship. Figure 4 presents the final thematic map for this analysis, to illustrate the relationships between themes.

\footnotetext{
${ }^{31}$ The title of the first theme is a direct participant quote. I named the remaining themes myself.
} 


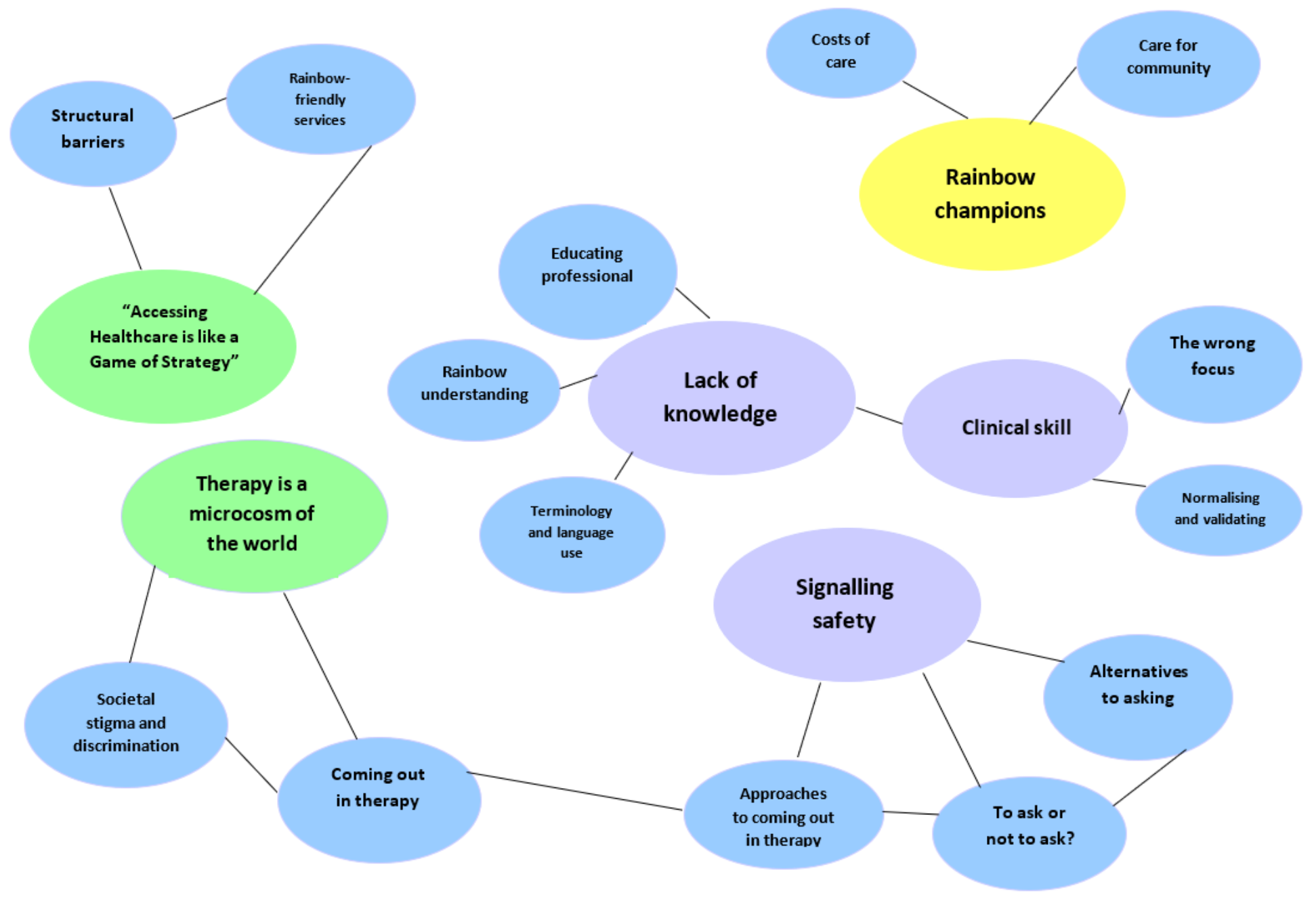

Figure 4. Final thematic map for analysis of interviews about accessing mental health support. 


\section{Thematic Analysis}

\section{Theme One: "Accessing Healthcare is like a Game of Strategy"}

This theme encapsulates participants' talk about barriers to accessing mental health support. As discussed in Chapter 2, New Zealand's public mental health services underwent significant funding cuts in the years leading up to this set of interviews, creating a system widely described as 'in crisis' (see, for example, Campbell, 2017; McAllen, 2016; Wright, 2016). When asked what had made the process of accessing mental health support easy or hard, almost all participants focussed on the structural barriers associated with seeking support. ${ }^{32}$ Most participants viewed New Zealand's mental health system as underfunded and overstretched:

Arthur: I think we've got a problem in this country when it comes to mental health support, I mean I think the whole thing's in crisis (...) I think that the struggles of those, of that collapse is being felt by people on the ground every day.

Wallis: I saw GP a few weeks ago where we were asking about getting a referral to a psych and he was like "there's no chance in hell" (laughs) I'm like, okay! (...) He's like, "did you see the article in the Herald the other day about how mental health services are so bad in this country?"33

Arthur and Wallis' comments present a gloomy view of New Zealand's mental health system in which access to care is, for some, a near impossibility. Wallis' laughter when recalling their conversation with their GP highlights the absurdity of their situation; within a publicly funded system, where access to care by those in need should arguably be guaranteed, Wallis was turned away by the very person supposed to provide them with support. Their experience suggests that healthcare providers are cognisant of the limitations of New Zealand's mental health system, however it was disappointing to

\footnotetext{
${ }^{32}$ I discuss the experiences of those who did not report structural barriers on page 73.

${ }^{33}$ Extracts are not numbered throughout Chapters 6 and 7; I use page numbers to direct readers back and forth as needed.
} 
hear that Wallis' GP did not respond with empathy or use this as an opportunity to advocate for Wallis' access to mental health support.

\section{Structural barriers}

The most commonly cited barrier to accessing mental health support was the length of waiting times. Participants described waiting times of up to six weeks for an initial appointment at a public mental health service and waiting times of up to three months for university counselling services. Waiting for appointments was typically described as distressing and frustrating, with participants noting that a lack of mental health support impacted on all areas of their lives. Arthur explained that he would likely have ended his life if he had not ended up accessing therapy privately:

Arthur: There was a period of time after my first year at university where I basically just crashed. I was in a very bad place, and I tried to access mental health support through the university, and I don't want to trash talk them at all, because I know that they have serious constraints, but I was really desperate and they said that I would be put on a three month waiting list, at the time where I probably would not have survived three months, and I'm really lucky in the respect that [my mother] ${ }^{34}$ was able to find me somewhere private to go.

Arthur's experience demonstrates the potentially devastating outcomes of lengthy waiting times in mental health services. His story of seeking support at a crisis point, rather than when he had first begun to feel distressed, was common. Several participants reported delaying help-seeking as they were aware they may not meet the criteria for publicly funded mental health treatment. ${ }^{35}$ Participants reported having to convince providers that they were very desperate in order to get help, and many had previously had referrals rejected: Lily noted that "I always get told that I'm not crazy enough for mental health services", while Charlie said that "It's been me trying to seek out kind of help and then sort of being on the cusp of being really sick but not quite sick enough to get access to services". That participants often put off help-seeking until they were "sick

\footnotetext{
34 Throughout the analysis I enclose information in square brackets either to maintain participants' anonymity (as in this case), or to provide context or clarification when needed.

${ }^{35}$ For many publicly funded mental health services, the criteria for treatment is 'moderate to severe mental illness or psychological distress' (Healthpoint, 2019).
} 
enough" highlights the need for more accessible mental health services, so that those in need can receive help before their difficulties escalate.

In addition to lengthy waiting times for accessing support, participants often reported that the number of sessions offered to them through public mental health services were inadequate to address their difficulties; Bonnie, for example, noted that her interactions with public mental health services were "very brief, I didn't really feel like we were getting there, which is what I needed because I've got like very long standing stuff." Participants were commonly offered six sessions of therapy; however, research suggests that to achieve clinically significant change the majority of clients with moderate to severe mental illness would need to remain in treatment for approximately 20 sessions (Harnett, O’Donovan, \& Lambert, 2010). This suggests that a key challenge seems to be a mismatch between mental health services' expectations of the treatment needed and the actual needs of clients accepted into their services.

Participants commonly shared that they had invested a great deal of time and effort into the process of accessing mental health support. Here, Star recounts her attempts to access counselling after immigrating to New Zealand:

Star: I've been trying to access healthcare for [trauma] for months, and that was really really difficult. I would say I got ghosted from a couple different places? (...) I contacted so many people, they kept telling me you know oh we have this kind of waitlist, this kind of waitlist, the [women's organisation] here had like 8 weeks waitlist, they just told me please go contact these people and these people and these people, [youth service] asks me for more information, I give them more information and they completely stop responding to me. The other two places I respond to one of the emails bounces cause it doesn't exist, the other one goes, I'm out of the office and I'll get back to you sometime.

Star uses "ghosted", an expression generally used in dating contexts to describe the dissolution of romantic relationships, to describe her experience of seeking mental health support. "Ghosting” refers to avoidance or withdrawal strategies enacted via technological mediums, such as ceasing to answer calls, or messages over text or social media (LeFebvre, 2017). Star explains that in more than one instance she was left wondering how long she should wait before reaching out to another organisation - an uncertainty that compounded her distress. 
Some participants reported that they had accessed mental health support privately, in the hopes of avoiding the challenges and pressures they had previously experienced in the public system or had heard about from others. A few participants had done so at a detriment to their savings, while others had accepted support from their parents:

Juno: That was me independently sourcing her and paying her out of my own pocket, so it was quite an expensive process as a student, to be doing that twice a week.

Cleo: I felt so bad for my mum, even though she obviously had no hesitations about me doing it, it was $\$ 100$ an hour, and that obviously pushed me to think, is this really working, and when I decided it wasn't I couldn't stay there because my mum was paying for something that I didn't think was helping.

Cleo's experience highlights that accessing private support can have an emotional cost, as well as a financial cost. Cleo thought her mum was paying a large sum of money for her mental health care, which led to feelings of guilt and a need to ensure that therapy was "really working". Had Cleo accessed publicly funded care she might have felt less pressure for her therapy to "work" within a particular time frame, and, subsequently, a longer period of engagement with mental health services might have increased the likelihood of Cleo finding the support helpful.

For the majority of participants, accessing private mental health support was not a viable option. Accessing publicly funded mental health support presented additional challenges for participants on low incomes or benefits. Bats, who was receiving a benefit for those living with a health condition at the time of the interview, was eligible for a Work and Income weekly disability allowance, which can help to cover the cost of therapy. In this extract, they describe the process of applying for this payment to fund treatment at a local anxiety clinic:

Bats: For the anxiety clinic, my support worker drove me there, got the forms, and then helped me make the WINZ appointment, and then made sure that was all filled out right, and then made me go back to the place to get something signed, and then another appointment to hand in with WINZ, and then I go back to the place, and then they're like, okay time to sort an appointment, and it took 
a really long time for the time for the anxiety clinic to start. A lot of it is waiting and juggling funding (...) I got the maximum disability benefit, which is like $\$ 62.50$ a week, and then I would see the anxiety clinic every two to three weeks, because two weeks of the disability benefit is not enough to cover one session every two weeks, so I played with times a bit to see how much I could go there without myself having to pay.

Bats' story highlights some of the hidden costs of accessing publicly funded support. Although the sessions themselves were funded through a disability allowance, Bats had to pay for public transport to travel between Work and Income and the anxiety clinic or ask their support worker to drive them back and forth. As such, accessing support either created a financial burden, or took up time with Bats' support worker, which was limited to a few hours each week. Hodgetts and colleagues discuss this 'running around' as one of many punitive welfare reforms that "pathologise the poor" (Hodgetts, Chamberlain, Groot, \& Tankel, 2014, p. 2036); here, the onus is on Bats to facilitate funding for their own treatment. Bats went on to describe deciding to cease treatment at the anxiety clinic, as they felt the effort required to access therapy outweighed the quality of support they received.

It is important to note that, while almost all participants had come across structural barriers at some point while accessing support, participants also spoke of instances where there were few or no barriers to access. Participants who had sought support with little difficulty had either paid privately for mental health care or had accessed services at their school or tertiary institution. For example, Cleo described her school counsellor as "amazing, in the sense no one knew I was going, not my classmates or my teachers or my parents, cause it was completely free, it was all down to me." Andie noted that "[in] institutions and places that I've worked or studied mental health services or just health services in general are quite accessible," and Tui commented that accessing counselling through her university service had been "relatively easy." When I asked Andie and Tui what made the services easy and accessible, they described short waiting times before (and between) appointments and noted that on-campus services meant that they did not have to pay for travel in order to access care. Andie and Tui's experiences emphasise that practical factors (such as 
geographical proximity) should not be underestimated when considering service accessibility for those in need of support.

\section{Rainbow-friendly services}

Although the structural barriers participants discussed seem to be a product of an overstretched and underfunded public mental health system, rather than participants' rainbow identities, difficulties in accessing support are seemingly exacerbated among queer, trans, and intersex New Zealanders due to the limited number of explicitly rainbow-friendly services. Star reflects:

Star: I guess part of the problem is that trying to find a service that focusses on or is at least receptive to LGBT issues means that you have a very narrow scope of places that you can reasonably go to.

Almost all participants stated that they explicitly seek out services that are rainbowfriendly when accessing mental health support:

Dean: Yeah, it's really important, I think, to have someone who's queer friendly, just cause I feel like queerness (...) is really important for identity so any kind of engagement with mental health services would have to be good with that, you know, even if it wasn't particularly related to sexuality, I think it's really important.

Martin: Often it's not exactly what we're there for but it informs a lot of our life and our decisions and our relationships, and you know our living circumstances and our safety and all of those things.

Juno: I want someone who is going to understand and respect the fact that I'm intersex.

Here, participants explain that because their rainbow identities are central to who they are as people, receiving support from services and professionals who affirm and validate these identities is crucial. Implicit in these extracts is an acknowledgement that not all services are necessarily rainbow-friendly, or receptive to discussing rainbow issues. I return to this view of mental health settings as potentially unsafe spaces in the next section of my analysis (see pages 76-84). 
When I asked participants how they went about finding rainbow-friendly services, the most common response was by word of mouth:

Rosie: You know I'll ask communities like "who do you see, who's good?" so I think, unless it's explicit in their kaupapa somewhere, it'll have to be word of mouth.

Naomi: I would ask around my friends in the community, you know be like who have you been to, who did you think was good, who did you think was bad.

Lesley: I think word of mouth is a little more trustworthy, just because you get that kind of like first-hand knowledge of how they reacted to specific things, whereas kind of like, a lot of people will put "queer and trans friendly" after going through like one workshop on queer and trans patients, stuff like that, I think it's just better knowing that someone else in the community has been to them, and likes them, and knows that they aren't shit.

Many participants reported that they had difficulty finding rainbow-friendly services through methods other than word of mouth, or, like Lesley, were unsure of whether they could trust services that advertised themselves as "queer and trans friendly". It seems, however, that the 'word of mouth' method of finding safe services has unintended consequences. Because New Zealand does not have any publicly funded rainbow-specific services, services that build positive reputations among rainbow community members tend to be general health services with staff who are particularly knowledgeable or passionate about rainbow issues:

Lesley: It's so interesting how that just happens, and it's kind of a little bit organic, like one person finds out about this really good doctor, so more people start going, then all of a sudden everyone goes there. Like we have a dedicated sexual health clinic out in out at [suburb], but I'm sticking with [my provider].

When word spreads about these services among rainbow communities, more sexuality, gender, and sex characteristic diverse people seek support from them. This puts additional pressure on their (often under-resourced) service, exacerbating issues such as lengthy waiting lists. In some cases, services are forced to close their books to avoid being overwhelmed by demand (Kondou, 2017). 
During interviews, services that participants most commonly described as rainbow-friendly were Youth One Stop Shops or Health Hubs, which provide integrated physical, mental, and social care (Asarnow, Rozenman, Wiblin, \& Zeltzer, 2015). Reviews of integrated care services demonstrate that, like the participants in the current study, young people are highly satisfied with the care they receive from these services (Asarnow et al., 2015; Hetrick et al., 2017). Several participants pointed out that they would soon age out of these services, and expressed concern that they would not be able to find another funded mental health service that was rainbow-friendly: Star, for example, asked "when I'm not eligible for youth services where do I go, what's my next step, what is there for the intermediate age for gay and trans people, you know?" This highlights the importance of upskilling in the areas of sexuality, gender, and sex characteristic diversity across all mental health services, rather than focussing solely on youth services.

\section{Summary}

The majority of participants encountered structural barriers when attempting to access mental health support. They reported long waiting times, rejection of their referral by services after not meeting criteria for treatment and having to expend time and effort approaching multiple services before gaining access to care. Participants described this process as exhausting, frustrating, and disheartening. While there is evidence to suggest that these experiences are common in the context of New Zealand's oft-cited mental health crisis, the desire to receive support from rainbow-friendly services intensified the challenges of accessing mental healthcare, as participants felt that this substantially narrowed the pool of potential health services.

\section{Theme Two: Therapy as a Microcosm}

In this theme participants described the stigma they face in their daily lives and reflected on whether mental health settings are safe spaces outside of a discriminatory societal context, versus potentially unsafe spaces embedded within it. For the most part, participants described coming out as risky within the therapeutic context, suggesting that they understood the mental health support setting as a microcosm of the world; that is, as a place in which they risk exposure to discrimination.

\section{Societal stigma and discrimination}


Although interview questions around stigma and discrimination were specific to experiences within mental health support services, almost all participants discussed fears, or experiences, of sex-, sexuality- and/or gender-based discrimination outside of therapy. This included stories of participants being bullied at school by other students (and, in one case, by a teacher), being verbally harassed by siblings, other family members, or strangers, having homophobic landlords, and experiencing discrimination within the workplace. One participant had been threatened with a weapon after a partner's parent had learned of their relationship, and another had lost her job after coming out as trans:

Alexis: Our gender expression changes the way that we're treated in society. I experienced a lot of prejudice in my previous workspace, and I lost my job inevitably because of it. I don't have legal protections for that in New Zealand ${ }^{36}$ so I couldn't do shit, and I was unemployed for six months. I would go out and people would question my gender everywhere I went, it was this thing, you can't escape it, it's everywhere and if you don't pass, you're fucked.

Alexis describes cissexism as inescapable, and highlights the dangers of being visibly trans, or not 'passing'. Her experiences, together with those of other participants, calls attention to ongoing queerphobia and transphobia in New Zealand. Charlie emphasised the importance of recognising the existence of this discrimination, noting that "with so many recent legal and media gains surrounding sexuality and gender, people outside that, or who don't experience significant discrimination, feel like everything's easy now and it's not." Participants' discriminatory experiences are consistent with recent literature suggesting that rainbow community members around the world continue to experience discrimination and violence (e.g. Burton et al., 2013; Friedman et al., 2011; James et al., 2016), despite ostensible improvements in rainbow safety and inclusion.

For several participants, concern about homophobia and transphobia was centred on their physical safety, and was ever-present; Felix, a trans man, described worrying about his safety "literally every time I meet anyone new, or go to a public bathroom, or

\footnotetext{
${ }^{36}$ As noted on page 11, the Human Rights Act (1993) does not prohibit discrimination on the basis of gender, although the Human Rights Commission interprets non-discrimination on the basis of sex to include gender identity (Human Rights Commission, 2010).
} 
leave the house." Participants from small towns and rural areas were particularly concerned when visiting their hometowns; most participants from small towns had relocated to larger cities, which they viewed as safer and more rainbow-friendly. Penny, a trans woman from a farming family, explained:

Penny: I'm going [to my hometown] later today, and it still makes me nervous being [there] because I'm terrified I'll run into people who I guess knew me, rather than know me. I was bullied throughout high school, bullies I know still live there, and some of them have gotten far worse since high school, so it's scary.

Arthur reported similar feelings of fear and worry when reflecting on his experiences in a Southland high school, sharing that "being around straight men in those environments was dangerous. I can't put it any other way, it was very dangerous." While there is a dearth of research about the experiences of rainbow community members outside of New Zealand's major centres, Arthur and Penny's experiences closely reflect prior research conducted in the US suggesting that queer and trans people in rural contexts face increased stigma, as well as social isolation and decreased access to health services (Fisher, Irwin, \& Coleman, 2014; Whitehead, Shaver, \& Stephenson, 2016).

\section{Coming out}

The impact of societal stigma and discrimination on participants' daily lives was most apparent during discussions of the ongoing coming out process. Participants typically framed coming out as something to be actively negotiated, with many commenting that there was always a possibility of a negative reaction. Rosie stated that telling others about her sexuality "can be a risky disclosure," while Star noted that "you never totally know how it's going to go, no matter who you're talking to." Naomi and Cleo were particularly wary about coming out or displaying their relationships in public settings:

Naomi: A few years ago, I was dating a girl and every time we were in public and I went to hold her hand like I would look around and see who was around and you had to make a call like really quickly and then quite often like the moment had passed and you hadn't like the opportunity to scan the area. 
Cleo: It's a daily thing, I mean walking down the street holding your girlfriend's hand or a guy holding his boyfriend's hand, I mean for me it's terrifying, and I've been with girls who have said the same, thought the same, and we just haven't done it, and that's very sad, because we want to, but we're way too scared about someone saying something.

Cleo went on to say that she had come out as gay to her friends in high school but had yet to share her sexual orientation with her new university friends, more than a semester into the first year of her degree. She explained that "every time I see them, I think, I'm hiding such a huge part of my life, and at the moment I'm single, but if I wasn't, I would want to tell them." Cleo seemed to be caught between a desire to share an important part of her life with others in her life, and a need to protect herself against potential discrimination. As Meyer (2003) notes, the need to hide sexual orientation for fear of discrimination creates a significant cognitive burden that contributes to minority stress, increasing risk for mental health difficulties.

Despite my familiarity with literature around stigma, discrimination, and the difficulties of coming out, as well as personal experience supporting friends through this process, I felt unsettled by the injustice of participants having to constantly consider whether it was safe to share their sexuality or gender with others. I was also struck by the matter of fact fashion in which Cleo, and many other participants, described the risks associated with coming out:

Cleo: When you discover your sexuality, being scared and being discriminated against is just something that comes along with it, it's not a maybe I will or maybe I won't, everybody in their life will meet someone who doesn't like it, or says something rude, it's just something that's gonna happen.

Cleo went on to explain that she could not imagine a world in which she could kiss a partner in public without anyone noticing or caring, saying she wished it was not so foreign to her, "but it's just the way it is I suppose." Tui reflected on this inevitability of worry during our interview, after I explained that I had been taken aback by the way most participants described concern around coming out as unavoidable, or as a fact of life: 
Tui: Yeah, it is normalised, it's funny, I think the reason I have to ask you to clarify is because I was like yeah! Of course I worry, but it is sort of like a constant (...) you know it just adds up or it's almost like a buzzing that's in the back of your mind constantly, you just don't notice it's there after a while.

Tui's description of worry about sexuality as a constant buzzing in the back of her mind mirrors Nadal and colleagues' (2011) notion of sexual orientation microaggressions as "death by a thousand cuts" (p. 234) and is another powerful illustration of Meyer's (2003) minority stress model. ${ }^{37}$ For Tui, a heteronormative society - in which "people never react positively [to my bisexuality], it's either like sort of neutral or negative" - is stressful. This accumulates over time, negatively impacting her mental health. I elaborate on the relationship between participants' identities and mental health in the 'lack of knowledge' section of this chapter (see pages 96-105), as I discuss mental health professionals' understanding of the complex ways in which societal stigma and discrimination create a hostile environment for rainbow community members.

\section{Coming out in therapy}

Following discussions of queerphobia, transphobia, and the ways in which participants navigated the coming out process in their daily lives, I asked participants about their experiences of coming out within mental health support settings. I expected that coming out in therapy may have been difficult for many participants. In the research described in Chapter 3, rainbow community members routinely describe coming out in therapy as stressful and anxiety provoking, due to fears that mental health professionals will lack understanding, or will respond negatively (Daley, 2010; Evans \& Barker, 2010; Hiestand et al., 2007; Hunt et al., 2006; Shelton \& Delgado-Romero, 2011; Sperber et al., 2008).

At the same time, I (a clinical psychology student in the early stages of training) understood mental health settings as places in which clients were not subject to the same rules that they were in society. Contemporary talk therapy is, for the most part,

\footnotetext{
${ }^{37}$ As discussed in Chapter 3, the minority stress model posits that societal stigma and discrimination create a hostile and stressful environment for rainbow people, which in turn increases risk for developing mental health problems (see pages 21-22).
} 
informed by Rogerian principles of warmth and positive regard, where mental health professionals accept and support their clients within the therapy context, regardless of what they do or say (Tolan \& Cameron, 2003; Tryon, 2010). As such, I thought it likely that participants would describe coming out in mental health settings as easier than they did in their day-to-day lives. However, participants shared the same concerns about coming out in mental health support settings as they did about coming out in other contexts:

Wallis: I'm happy to talk about my mental health stuff, and the nitty gritty of that, but as soon as it comes to the queer stuff, I'm like step back a bit, and I kind of guard it a bit more carefully (...) you know it's a safe space, but it's still kind of "can I actually share this here, how much can I share, what am I being judged on" cause like even if they're a counsellor they've still got opinions about it, whether they can share them or not.

Andie: It's definitely scary no matter where you go, cause you don't know what everyone's perception on gender is, you don't know what everyone's perception on sexuality is, it's kind of unpredictable and with seeing mental health services when you're seeing somebody new it's quite scary so yeah I was scared, panicking and fretting about it for a long time.

Keith: Even support workers and counsellors and therapists, they might be homophobic and you don't know. They might also be one of those people who are like "oh you know, all these gay people think it's all about their sexuality, it's not as bad as it was 30 years ago" and that sort of thing, and it could be a problem.

Like participants in previous studies (e.g. Edwards \& Van Roekel, 2009; Lucksted, 2004; Poteat et al., 2013), Wallis, Andie, and Keith report a wariness to talk about their identity in mental health settings, due to the possibility that their understandings of sexuality and gender will conflict with those of their mental health professionals. Wallis and Andie were particularly concerned that their professionals would view gender as fixed and binary and would deny the reality of their nonbinary gender identities. Other participants made similar comments about the diversity of viewpoints that mental health professionals hold, noting that some are likely to have pathologising or old-fashioned ideas about rainbow identities. Participants also 
emphasised that professionals often refrain from sharing their views around sexuality, gender, and sex characteristic diversity with their clients - a potential heuristic for rainbow service users to identify safe and validating professionals.

Participants described a multitude of worries in relation to coming out in mental health settings, including that mental health professionals might respond awkwardly, judge them negatively, fail to understand their identity, see their identity as deviant, suggest their mental health difficulties were a result of their sexuality or gender, or ask invasive questions (for trans participants, invasive questions often related to hormone therapy and surgery). Taylor described concerns that her therapist might break confidentiality if she was to come out:

Taylor: I was ready to talk about [sexuality] and I didn't know if that was a safe place or if she was gonna go and - I know this sounds ridiculous - but out me to my parents, or like I didn't know what the ramifications of coming out were going to be.

While most participants were cautious about coming out in the therapy context, some participants described disclosures of identity as comfortable within mental health settings. Bonnie explained that she was very up front about her sexuality in therapy: "I'm very firmly confident in that part of myself, so, I'm literally like hey! I'm queer, are you cool with that?" Naomi noted that she did not tend to worry about coming out in therapy as she felt able to make a complaint "if they have an issue with it," while Alex commented that he felt he "had enough of a defence that I could actually stand it if bringing it up went wrong." Importantly, Bonnie, Naomi and Alex did not describe coming out as safe within mental health settings, but acknowledged that there was a possibility that coming out would be met with a negative response, and felt that they had the resources to cope with such a reaction.

\section{"Our mental health system is indicative of our entire society"}

Together, participants' talk around coming out in mental health settings suggest that they viewed mental health support settings as a microcosm of the world. Rather than seeing therapy as a safe space, or a context in which their identities would invariably be accepted and validated, participants described mental health settings as places in which they may be exposed to the stigma and discrimination they routinely 
experience in daily life. Some participants explicitly reflected on the embeddedness of therapy within a homophobic and transphobic society:

Taylor: I think that our mental health system is indicative of our entire society, and my experience of being a closeted and then out gay person in our society is completely indicative of mental health. Like there kind of is no explicit difference in terms of the way the culture of our mental health system is the same as the culture of our society, and so like all the same rules apply.

When I asked Taylor what the "rules" were, she explained that, in both mental health settings and the wider world, people (or mental health professionals) either maintained a complete silence around her sexuality, or made it the sole focus of conversation at the expense of other important aspects of her life: "It's like overcompensating or nothing."

Alex made similar comments about the ways in which mental health settings reflect the wider world, stating that "It's difficult because despite all efforts to the contrary, you're not just sitting there on your own in the room with the counsellor, you're sitting there in a context of a homophobic and transphobic society." Alex went on to reflect that this social context of heteronormativity and homophobia may be more powerful than any actions on the part of this counsellors:

Alex: The difficulty with that is to be absolutely honest my counsellors have given me no reason to think that they would be likely to misconstrue [my experiences]. But, nevertheless that social context exists and so it's really hard to talk about that. On the other hand, like there is something to be said about the counsellor's room, you know, that I'm able to talk, I do feel more free to talk about things (...) there's more open space to talk about divergences from the script. At the same time, it's still there, it still has an influence over what I am and am not able to say.

Here, Alex notes that the safety of the counsellor's room does make it easier to talk about his experiences of sexuality than in other social settings. This contrasts with the ways in which many other participants framed mental health support settings, as potentially unsafe and invalidating. When Alex mentions "the script", he refers to mainstream or uncritical understandings of sexuality, gender, and sex characteristic diversity, much like those that Andie and Wallis discussed earlier in this section (see 
page 81 ). He notes that the homophobic and transphobic societal context limits his ability to challenge these understandings in therapy, despite his counsellors' openness and safety.

The finding that rainbow clients articulate therapy as a microcosm of the wider social context indicates that they are likely to be wary of mental health professionals, regardless of the professional's level of safety and competence, as a result of living in a hostile social context. It is also important to consider that participants in the current study were often aware of the pathologising history of mental health care and had either heard of negative mental health support experiences of others in the community or had negative experiences themselves. These factors contribute to a sense of distrust or wariness of mental health professionals and highlight the need for mental health professionals to actively challenge heterosexism and cissexism, ${ }^{38}$ and to communicate this to their clients.

\section{Summary}

Almost all participants in the current study shared that they had, at some point in their lives, experienced discrimination on the basis of their rainbow identity. Living in a discriminatory society often complicated the coming out process; participants were typically wary of coming out in contexts in which their safety might be at risk, or where they might receive negative reactions from others. Importantly, the majority of participants described mental health support settings as one of these risky contexts, demonstrating the need for mental health professionals to take an explicitly rainbowaffirmative stance. Of course, like many aspects of effective mental health support provision, creating a rainbow-friendly therapeutic space may be easier said than done. The next theme explores what an explicitly rainbow-affirmative stance might look like in practice.

\footnotetext{
${ }^{38}$ Actively challenging discrimination is also consistent with Principle Four of the New Zealand Psychologists' Code of Ethics (New Zealand Psychologists Board, 2002): Social Justice and Responsibility to Society.
} 


\section{Theme Three: Signalling Safety}

This theme elaborates on participants' experiences of coming out in therapy, with a particular focus on professionals' silence around sexuality, gender, and sex characteristic diversity within mental health settings, and talk about whether participants think that mental health professionals should ask direct questions about identity. As discussed in the previous section, participants were often wary of coming out in mental health settings, due to concerns that mental health professionals would respond with judgement, discomfort, or inappropriate questions. For many participants, the process of coming out in therapy was further complicated by the fact that mental health professionals rarely asked about rainbow identities, or whether sexuality, gender, and sex characteristic diversity was something they wanted to discuss. Participants generally reported that mental health professionals had only initiated conversations about identity in cases where clients were presenting for gender-affirming healthcare or had information about their identity included in their referral. Taylor noted that "nobody asked [about sexuality], up until I brought it up," while Theo said that "when you're just there for what they think is general reasons ${ }^{39}$ they don't ask [about gender] at all."

Professionals' silence around identity left the onus on participants to decide whether to come out in therapy, and how to do so. For most participants, the benefits of disclosure outweighed the potential risks, and they usually chose to share that they were part of the rainbow community. For example, when I asked Star (who is agender, and previously identified as a trans man) if it was important that her rainbow identity was acknowledged in therapy, she responded:

Star: Yeah, it's really weird if you dance around it, especially because we're talking about a timeline of my history, and we can't do that without acknowledging that for a huge chunk of my history I was this guy, you know, instead of this girl, or whatever I am (laughs).

Star's laugh and reference to "whatever I am" was an acknowledgement that her gender is, at times, confusing for her, so it was likely that her therapist might also be confused

\footnotetext{
39 Theo used "general reasons" to refer to common presenting problems within mental health settings, such as depression and anxiety.
} 
by her gender journey. She emphasised, however, the importance of taking the time to share the history of her gender in therapy, in order to tell a coherent story of her life and experiences. As mentioned in the first section of this analysis (see page 74), participants typically viewed their rainbow identity as central to who they are as people. When participants' providers did not know they were queer, trans, and/or intersex, this limited what they were able to discuss in mental health settings. Because of this, coming out was often framed as essential groundwork for successful therapy.

\section{Approaches to coming out in therapy}

Participants used a range of approaches to come out in mental health settings. Some participants disclosed to mental health professionals that they were part of the rainbow community by weaving talk about their identity into stories or mentioning partners or ex partners - "I just casually drop in 'my partner [name]' or 'my girlfriend' or whatever" (Rosie). Other participants tested the waters with their mental health professionals, looking for signals that it was a safe space to come out before doing so:

Hugo: Sometimes I'll disclose [my gender] and sometimes I won't (...) I'll choose depending on if I think the person's chill and I'll usually drop a gay joke in there first to see if they're okay with gay and if they're okay with gay then maybe they're okay with trans.

Here, Hugo suggests that acceptance of marginalised groups tends to cluster together. This was echoed by Star, who noted that if her therapist is "okay with me being gay, that doesn't always mean she's okay with gender stuff, but it gives me a better chance." Hugo and Star's use of "maybe" and "chance," however, communicates that coming out as trans is still risky; they remain uncertain that mental health professionals will accept and validate their diverse genders. Several other participants described a similar process of scoping out their mental health professionals before coming out - Bats noted that they are typically open about their nonbinary gender, but "I would obviously gauge the situation beforehand," while Lesley said that "I'd probably take some time to chat with them before deciding whether to come out, trying to gauge that reaction."

Some participants took an alternative approach to the scoping out method by disclosing their identity up front, in the first session with a new mental health 
professional. They then used the professional's response to guide decisions around continuing or ending the therapeutic relationship:

Kit: I was like oh I'm just gonna test this out from the beginning, so I'm Māori, non-smoker, bisexual.

Eli: What I usually do is drop something in about it in the first session, cause then I'm interested to see their reaction. I do that as a way of seeing if they're good on that (...) so I'll talk about having sex with men, that's usually the opener.

Norah: There is that moment where I'm like "okay, I'm gonna bring this up now and see how they respond" cause there's no way that I could proceed if that was an issue.

As well as reiterating the potential for discrimination within mental health settings, Kit, Eli, and Norah's talk about "testing it out", "seeing how they respond" and "seeing their reaction" highlighted that coming out in mental health settings is not an organic process, or a topic of conversation that comes up naturally. Like participants in previous studies (e.g. Evans \& Barker, 2010; Hunt et al., 2006), interviewees described coming out as something to be actively negotiated; they continuously attended to signals of risk versus safety to guide their decision-making. Participants also emphasised that 'coming out' in mental health services is only relevant within a heteronormative and cisnormative society which assumes everyone as straight and cis:

Kit: I dislike the words around 'coming out' cause it always sounds like you were hidden, or you were hiding, or lying, or those sorts of things and I dislike the connotations because other people are making assumptions that 'you must be of the norm until you tell me otherwise.' And even that my sexuality has to be something that I need to go round announcing.

Here, Kit expresses her frustration about the supposed 'need' to come out. She notes that she has to "go round announcing" her sexuality so that others do not read her as straight, then hold her accountable for their incorrect assumption. Consistent with Semp's (2006) analysis of heteronormative discourses, Kit went on to reflect that the ability to be silent about one's identity is a sign of privilege, and noted that if sexuality, 
gender, and sex characteristic diversity were acknowledged in society, she would be relieved of the pressure to repeatedly tell others of her takatāpui identity.

In discussions around coming out in mental health settings, participants also stressed that they pay close attention to professionals' reactions when they disclose their rainbow identity. Participants typically stated that they appreciated professionals acknowledging their disclosure and checking in if they would like to discuss it further. When professionals appeared surprised or uncomfortable, participants often interpreted this to mean that they had assumed them to be straight and/or cis and were taken aback when they learned this was not the case. Naomi noted that "the lack of surprise when you disclose a sexuality that isn't the norm, that absence of broken expectation does so much for an atmosphere." Kit described her ideal reaction as "respectful indifference" that is, the professional does not focus unnecessarily on her identity when she brings it up, but also respects that coming out (both in the therapy context and in general) may have been significant for Kit, so must be acknowledged.

\section{To ask or not to ask?}

Several participants saw mental health professionals' silence around gender, sexuality, and sex characteristic diversity as evidence that their professional had made assumptions about their identity, or was not accepting of rainbow community members:

Andie: If you're there to talk about your gender identity or sexuality and then it hasn't been brought up, you start wondering "oh okay maybe that's not okay to talk about”.

Star: It's not really just the silence, it's the silence coupled with their assumptions, because that leads to a climate where you feel like "shit, they've already assumed I'm straight, and it's going to be weird if I correct it".

Here, Andie and Star suggest that, within a heteronormative and cisnormative context, professionals' silence around gender, sexuality, and sex characteristic diversity is not a neutral act. Rather, silence is indicative that a professional has accepted the status quo (hetero/cisnormativity) or has failed to consider that their client may not be straight and/or cis. Participants who supported asking about sexuality, gender, and sex characteristic diversity commonly argued that incorporating these questions into conversation would disrupt normative assumptions and would represent an 
acknowledgement on the part of professionals that their clients may be part of the rainbow community. This, in turn, would make it easier to come out. Naomi noted "I love it when [sexuality and gender] comes up, I'm like oh my gosh! You're acknowledging that this is a thing, that's great," while Jasper said he would feel "very respected" if he was asked about identity, "and like someone actually cared."

While questions about sexuality and gender were, for many participants, evidence that their mental health professional was rainbow-affirmative, others expressed reservations about asking these questions. A common concern was that asking about rainbow identities may put pressure on clients to provide an answer before they were ready. As mentioned earlier in this section, there was significant variation in participants' level of comfort in sharing information about their identity. Many participants considered their rainbow identity as factual information about themselves, akin to their address or occupation, and reported that they were happy to share this with others - "I don't mind if someone asks me like my pronouns or my orientation, that's all kind of fine and factual information" (Taylor). Some participants, however, only felt comfortable coming out to those they had built trust with. Wallis explained:

Wallis: Whenever I come out to someone I feel like I'm giving away a piece of myself, and sometimes I'm happy to do it and I trust them with that, but sometimes it feels like someone's just taken something from me. It's mine and it's so personal and integral to who I am and how I experience the world.

Here, Wallis explains that for them, coming out is not something that feels equally comfortable with all people. Rather, it is a meaningful process that can feel distressing if not done on their terms. As such, it is important that they are able to take control of decisions around coming out. Wallis acknowledged that being asked about sexuality and gender within mental health settings can be nice, "cause that wipes the board clean of any assumptions about things," but noted that there is also potential for a mental health professional to ask about Wallis' identity at a time when they are not yet comfortable sharing it. When asked about their sexuality, gender, and sex characteristic diversity in mental health settings, clients may be put in a difficult position of deciding whether to come out before they are ready, or to answer in a way that does not feel true for them (e.g. Wallis would write 'she/her' on an intake form asking for pronouns rather than their correct pronouns of 'they/them'). 
Participants expressed several other concerns about incorporating questions about rainbow identities into mental health assessments. A few participants pointed out that they prefer not to put labels on their identity, and that being asked direct questions about identity makes them feel like they must label themselves. Some participants noted that particular identity labels have stereotypes associated with them and expressed concern that mental health professionals may use clients' identity labels to form incorrect assumptions about them. Other participants questioned the rationale behind asking these questions:

Alex: You would assume that the counsellor only bring up things that they felt were relevant, and the problem is if the counsellor brings up sexuality first, I could see people feeling like that would be implying that their gender or their sexual orientation was the cause of their mental health problems.

Here, Alex points out that raising sexuality within mental health settings may imply a causal relationship between a client's mental health problems and identity. Given the field of mental healthcare's history of pathologising rainbow identities (detailed in Chapter 2), together with a societal silence about sexuality, gender, and sex characteristic diversity, it is unsurprising that rainbow clients may question professionals' reasoning behind asking about rainbow identities. Rosie, for example, who was supportive of asking up-front questions to begin with, noted later in her interview that "I would probably be wondering why they wanted to know. I wouldn't necessarily assume based on it being on the form that it wasn't gonna be used against me at some point."

For the most part participants initially took a firm stance on asking direct questions about rainbow identities in mental health assessments, however several participants went on to reflect further on the advantages and disadvantages of asking about sexuality, gender, and sex characteristic diversity, and often shared that asking about identity in mental health settings is a more complicated issue than they had originally thought.

Piper: I don't know about being asked because I know for some people that's really invasive and they don't feel safe (...) But at the same time it is nice to be asked, it's nice to have people not just assume that you're just straight or just cis, or whatever. So it's a tough one, I don't really know. 
Other participants also acknowledged the difficulties in deciding either way. Arthur, for example, noted that "When you ask that question, it can go really badly, but it can also create a safe space for people," while Tui described asking about identity as a "hard balance." Although I had advised participants that the goal of the study was not to produce definitive answers about the interview questions, many participants seemed frustrated that they did not have a clear preference for one option over the other.

Often, participants resolved this tension by emphasising the importance of context: "I don't think there is one answer, or a right answer, I think it's dependent on lots of factors" (Martin). The factors commonly outlined by participants included how comfortable the client feels about coming out, whether identity is relevant to their presenting problem, who the mental health professional is, and the manner in which they asked the question. Participants were, for example, more likely to support direct questions about identity in cases where the client had accessed support specifically to talk about rainbow issues or identity - "If a person is specifically wanting to talk about their sexuality and gender, then go ahead and ask their gender, ask their sexuality" (Theo). Participants also expressed they would be more comfortable with direct questions if they knew that all clients were asked the same questions, and if they felt comfortable with the mental health professional's wording, tone of voice, and body language.

\section{Alternatives to asking}

Participants' thoughts on asking about rainbow identity calls attention to the diversity of experiences within the rainbow community and highlights the importance of working flexibly in mental health settings; there are very few blanket rules that can be applied to work with rainbow clients (or any clients, for that matter!). In saying this, over the course of the interviews participants suggested a range of alternatives to asking direct questions about identity, which I have labelled 'safety signals.' Safety signals communicate to clients that it is safe to come out, or to discuss sexuality, gender, and sex characteristic diversity, but are more subtle than direct questions about rainbow identities. Interestingly, there was considerably less debate about the use of safety signals than there was about direct questions. While participants varied in the degree to which they thought these signals would be helpful, none were described as potentially harmful or confronting for queer, trans, and intersex clients. 
Checking pronouns and names. All participants in the study stated that they would feel comfortable if their mental health professional checked in on how they would like to be addressed, in regard to their name and pronouns:

Felix: When you first meet - "hey, do you have a nickname or a name that you prefer for me to use? Which pronouns would you like me to use?" Which would show that that specified mental health professional was LGBT friendly and considerate, and it would also make the person more willing to talk about it if it is an issue.

Star: At my old clinic I remember being so happy when I was introduced to my new GP and she was this tiny little old lady and she was like "so how should I refer to you?" and it was a pronouns question, you know, without explicitly being a pronouns question.

Dean: Asking pronouns, I think that's awesome, I think it's a really important thing, and that could be embraced by mental health kind of services.

Some participants also reflected on the fact that gender can be fluid, and that pronouns may change over time. Because of this, some mental health professionals check in around pronoun use regularly - one participant commented that their health service checked their pronouns at every appointment, which the participant found very frustrating. To acknowledge potential changes in pronoun use while avoiding unnecessary pronoun checks, mental health professionals could ask their clients to let them know of any changes to their pronouns or name.

Expansive language around relationships. Several participants reported instances of mental health professionals using gendered pronouns to refer to their clients' partners, before the client had confirmed the correct pronoun. For example, Star shared that in her intake phone call for a new counselling service, "the lovely old lady on the phone is asking me about, do you feel safe with her partner, and she uses 'he' and I just quickly go "she." Participants emphasised that the use of expansive language is key in communicating that no assumptions have been made about a client's identity:

Bats: I think you can tell if someone is understanding of the queer community in how they ask questions. If I was asked "oh, do you have a boyfriend?" then I'd be like, okay they're probably assuming that I am female and that I am straight. 
Tui: It would be nice if counsellors were always sure to say "oh, and any other partners?" and then use gender neutral pronouns until they know for sure, because there's just that immediate assumption that makes it really annoying.

Charlie: If somebody is talking about their partner don't just assume it's a heterosexual relationship until a person describes it like that.

Here, Bats, Tui, and Charlie emphasise the importance of asking open questions such as “do you have a partner, or partners?" or "are you seeing anyone at the moment?" to establish whether a client is in a relationship. Some participants liked the question "do you have a boyfriend or a girlfriend?" while others saw this as potentially exclusive of nonbinary gender identities. In the above extract, Tui stresses that counsellors should ask about other partners (past or present), in order to avoid assuming that a client's current partner is representative of all partners; as in Richters (1997) study, Tui, a bisexual woman, found that mental health professionals often assumed her to be straight when she talked of boyfriends or ex-boyfriends, and did not ask whether she had dated women in the past.

Several participants noted that discussions around relationships were a key opportunity for mental health professionals to signal safety to new clients. Juno described her first session with her clinical psychologist, who respectfully explored whether she wanted a relationship, and who she may want a relationship with:

Juno: She said I see here that you're intersex, tell me what it means for you to be intersex, so I was then able to say, from that, that I was part of the intersex community and I'd recently started identifying as intersex female (...) and so she said "okay, so you're an intersex female, so are you in a relationship?" and I said "no", and she said "are you interested in a relationship?" and I said "yes", and she said "okay if you were in a relationship, what sort of partners would you like, or do you think you may like to be in a relationship with?" so I said "I'm straight, I want to be with guys", so you know, she just built it, it wasn't like, "do you have a boyfriend?"

Juno describes the way in which, at every stage of the conversation, her psychologist made no assumptions about her identity. By asking Juno what it means for her to be intersex, Juno's psychologist acknowledged the diversity of the intersex community, 
and invited Juno to share where she sees herself in that community. Juno's psychologist went on to ask questions that did not assume Juno's relationship status, that she wants a relationship, or who she wants to be in a relationship with. Importantly, the question “what sort of partners would you like?" could refer to potential partners' gender, but could equally refer to their personal characteristics. Juno described the conversation as "very safe," and noted that they helped her to build trust with her psychologist.

Visual cues. A number of participants noted that they found visual cues an effective signal that a professional or service was rainbow-friendly. These included posters, flags, stickers, or buttons with rainbow colours and brochures or flyers about sexuality, gender, and sex characteristic diversity:

Norah: Those symbols do mean a lot if you're in the community, you wouldn't have that in your room if you weren't cool with it.

Bats: I definitely find I'm more likely to talk about that kind of stuff if they've got those like posters around, um, Sam Orchard's little gender sexuality posters, ${ }^{40}$ I'm just like oh they're trying to show that they're open about all that stuff, and maybe then I can be open with them.

Cleo: I've never been to anyone where there was an indication of support, which I think would have helped so much, just the poster on the wall, or something rainbow on their desk or something, just would make the world of difference.

These comments were part of a broader point participants made throughout the interviews; that within mental health contexts, small acts or gestures can have significant consequences. Participants noted that this was the case for making them feel both safe and unsafe. For example, a fleeting look of surprise when a participant came out was, for some, the end of a therapeutic relationship. The above extracts suggest that displaying something as simple as a rainbow sticker could send a strong message of support and validation to queer, trans, and intersex clients.

${ }^{40}$ Sam Orchard is a New Zealand-based queer and trans illustrator, comic creator and designer. Visit http://www.imlocal.co.nz/\#freestuff to access the Gender \& Sexuality 101 Comic and posters he designed for RainbowYOUTH. 
Institutional factors. Participants often described changes that mental health services could make at the institutional, rather than individual level. This included having all-gender bathrooms available at the service, and ensuring that intake forms were inclusive of all clients. Andie noted that "for me it would have been really reaffirming to have gender identification first, and pronouns first, and then sex assigned at birth after it." Several participants emphasised that forms should always have "prefer not to say' options, in case someone did not want to disclose aspects of their identity, and Hugo stated that it was unhelpful to add a third 'trans' box to existing 'male' and 'female' boxes. He shared that he does not tick the 'trans' box when he comes across forms like this - "I'm not the gender of trans, so I usually just write male."41

Advertising as rainbow-friendly. During my analysis of theme one, I briefly discussed the ways in which rainbow clients identify friendly services (see page 75). Although participants predominantly located services based on word of mouth, some expressed that explicit advertising as rainbow-friendly was, or would be, helpful:

Jasper: The biggest thing (...) was really the advertising as a queer friendly person. It's huge, I think. It's like being out as a queer friendly person, so that initially made me feel like there was a strong possibility that she would be a good fit for me.

Charlie: I actually spent a great deal of time going through profiles on doctor's websites and seeking reviews. It definitely would be important to me if people would communicate that they're sensitive about mental health or LGBTQ, like quite explicitly stating they are trans and queer friendly.

While Jasper and Charlie emphasise that explicit advertising can be effective in communicating that a service is equipped to work with queer, trans, and intersex clients, there was some evidence to suggest that services advertise themselves as rainbowfriendly prior to earning this title; Willow pointed out that "if they do say queer and trans friendly on the thing, there's no guarantee that they are actually going to be," while Eli noted "I have mixed feelings about sort of surface level stuff cause sometimes they

${ }^{41}$ For an in-depth discussion of how to ask questions about gender on surveys and forms, see Fraser (2018). 
can indicate that things are safe when they actually aren't." This highlights the importance of services undergoing training on mental health service provision for rainbow clients or taking time to seek education on rainbow mental health issues, before advertising as rainbow-friendly.

\section{Summary}

In discussions about coming out in mental health settings, participants reported that their mental health professionals typically do not initiate conversations around sexuality, gender, or sex characteristic diversity. Participants used varying approaches to come out in therapy, including discussing identity up front, weaving talk around their rainbow identities into stories, or 'scoping out' their mental health professional before coming out. There were a range of perspectives as to whether mental health professionals should ask questions around rainbow identity; while many participants felt that asking about identity would be normalising, validating, and would show that professionals had not made assumptions, others expressed that direct questions about identity would feel confronting, and may imply a connection between identity and mental health that queer, trans, and intersex clients would find uncomfortable. Despite disagreement about direct questions, however, participants agreed on a series of more covert 'safety signals' that could communicate a rainbow-affirmative approach, including checking pronouns, using gender neutral language, making forms inclusive, advertising services as rainbow-friendly, and displaying visual cues of support.

\section{Theme Four: Lack of Knowledge}

When discussing mental health support experiences, the single biggest concern participants expressed was the lack of knowledge they felt mental health professionals had about sexuality, gender, and sex characteristic diversity. Participants described knowledge gaps in the use of appropriate language and terminology, the issues faced by particular groups within the rainbow community, and the nature of the relationship between identity and mental health. Participants commonly reported that they had to educate their mental health professionals about rainbow issues and stressed the need for rainbow education and training within the mental health sector.

\section{Terminology and language use}


A number of participants reported that their mental health professionals lacked knowledge of rainbow terminology and had asked for definitions of the words clients used in session, including 'cis,' 'LGBT,' 'trans,' 'dysphoria,' 'pansexual,' and 'polyamory.' For the most part, participants were willing to define terms for their mental health professionals (especially lesser-known terms such as 'pansexual' or 'polyamorous'). They did, however, express frustration that their mental health professionals lacked knowledge of basic definitions and argued that looking these up would not be time consuming or difficult; Alexis stated that "they don't need to read huge research articles and papers and stuff about it, they can just watch a few damn YouTube videos.”

Several participants were particularly disheartened by mental health professionals referring to them using incorrect identity labels. Bonnie shared that her mental health professional consistently referred to her as "gay" after she had stated that she identified as queer or pansexual, while Felix recalled that, after coming out as a trans man to his school counsellor, the counsellor called a local mental health service and stated that she had a "young lesbian" with her in the room. Martin explained the importance of professionals matching clients' language in mental health services, as the choices that rainbow people make around language are not arbitrary:

Martin: I think our linguistic choices are crucial, particularly as minorities.

That's kind of how we relate to the world, by choosing certain words to describe us, our behaviours, our histories, whatever. I think it's really important to honour pronouns and names (...) if I call myself trans don't call me a transsexual. I think our word choices are deliberate and I don't like seeing other people mess with those.

Here, Martin stresses that, in a world where rainbow community members experience stigma, discrimination, and erasure, language is an area where queer, trans, and intersex people can take ownership of, and celebrate, their identity. Because of this, the difference between words like 'gay' and 'queer' are often meaningful to rainbow community members. Bonnie and Felix's experiences suggest that mental health professionals may, in contrast, view these distinct terms as interchangeable.

Although many participants emphasised the importance of language, it is important to note that they did not expect that professionals would invariably use 
correct terminology in mental health settings. Rather, participants acknowledged that mistakes can (and do) happen and focussed instead on professionals' response to their mistakes. Andie, for example, recalled an instance of her doctor writing an incorrect pronoun on a referral form - "I asked her is it okay if we can use they/them pronouns, she said 'oh absolutely! So sorry, I didn't realise' so that was good." Andie described the doctor's apology as sincere and was happy that the mistake had been acknowledged. When professionals did not acknowledge their mistakes, however, or attempted to account for them, participants described feeling frustrated and distressed. Hugo described an appointment where his endocrinologist addressed him by his deadname three times:

Hugo: I was really rude to him, but to be fair he deadnamed me, and like I told him not to and he did it two more times, and he's like "I don't know why you're being so hostile about it" and I'm like why are you so hostile towards me!

This extract suggests that Hugo's endocrinologist did not recognise the importance of using Hugo's correct name. For Hugo, a medical professional repeating his deadname after Hugo had called attention to the mistake was akin to saying that this is his correct name - that his chosen name is invalid.

Nala, a trans woman, described a similar experience to Hugo, in which she pointed out her therapist's incorrect use of pronouns:

Nala: She misgendered me once and then straight afterwards I called her out on it and then she said that she thought that there was a man and a woman in every person and started saying stuff like that, and then told me this story about how people often see her as a man, which really annoyed me.

Nala saw her therapist's response as an attempt to explain away or justify her misgendering and argued that her therapist's comments about gender were irrelevant; "I wanted to say, you didn't misgender me because you were seeing like my male side, what you're perceiving as male is not a natural male side, it's a facade." Nala brought the topic up again in her following session and said that her therapist did not seem to understand why it annoyed her. For Nala, her therapist using the wrong pronoun suggested that her therapist may not see her as a woman: 
Nala: At the beginning I did feel like she doubted whether or not I was a woman (...) if I feel like there's doubt there that kind of defeats the purpose of the therapy at all, because I want it to be a place where I can just say anything and say any doubts or any thoughts without feeling like I have to prove my gender. I want that to just be accepted no question so then I can just say whatever I'm thinking without feeling like they're using that to question whether I'm actually trans.

Nala's experience highlights the potential for language to invalidate (or validate) the identities of rainbow community members. It also calls attention to the fact that accounting for, or providing an explanation for, one's mistake (a habitual feature of language use; Potter \& Wetherell, 1987) may not be the appropriate response in cases of misgendering; it is difficult to imagine how Nala's therapist could have provided an explanation of misgendering in a way that did not intensify Nala's feelings of dysphoria. As such, while mental health professionals should endeavour to use the correct pronouns and identity labels to refer to their clients, in cases where they accidentally say the wrong thing, participants typically noted that they prefer their professionals to acknowledge the mistake, apologise, and move on.

\section{Understandings of sexuality, gender, and sex characteristic diversity}

In the course of the interviews, participants typically described sexual orientation and gender (and, to a lesser extent, sex characteristics) as socially constructed, nonbinary, and potentially fluid. These understandings were consistent with a queer theoretical approach (see pages 50-51), and challenged essentialist views of identity as fixed and biologically determined:

Bats: Everyone's gender is performative, whether you know it or not, doesn't make it any less real.

Willow: It's not a fixed thing, some people don't like find a solid identity for their entire lives, and that doesn't necessarily mean that they're failing at gender (...) there are a lot of people who are very fluid in their sexuality and or gender. Here, Bats articulates Butler's (1990) notion of performativity to explain that, to them, gender is not something that we are, but is something that we $d o$. They are careful to note that this does not make gender fictitious or unreal; rather, gender has a profound 
impact on Bats' lived experience. Similarly, Willow describes the potentially fluid nature of gender, and explains that there is no correct way to identify one's gender or sexuality; having a fluid gender and/or sexuality is a legitimate way of being in the world.

Many participants reported a mismatch between their own understanding and professionals' understanding of sexuality, gender, and sex characteristic diversity. Willow, for example, stated that "nobody knows a single thing about gender. I have known more about gender with like my half-arsed internet researching than like, every single professional that I've been to thus far in my life." Participants noted that their mental health professionals generally understood gender as binary, and often failed to differentiate between sex, sexuality and gender:

Nykk: Well, with my first school counsellor, she was really nice and really keen to research and learn, but she didn't really know anything, and she kind of viewed everything as the same.

Gloria: How do you mean?

Nykk: That gender and sex are sort of the same and such, and (...) it felt like I was really the one who was educating her and it felt really difficult, cause I wasn't sure.

Here, Nykk explains that their school counsellor assumed Nykk's gender based on their assigned sex at birth, and was confused when Nykk presented with questions about gender. Though Nykk now understands their gender as fluid, they reported that they were unable to articulate this at the time, and did not feel that their counsellor was prepared to support them as they explored their gender. Other participants described similar experiences when they attempted to reach out for support in navigating their identity; Piper recalled that while in residential care she began to think about her sexuality, "and there were a couple of support workers there who were like 'oh, nah you're not gay, cause you would know if you were gay." Juno noted that when she began to navigate intersex issues with her counsellor, "she really didn't understand what intersex was, and I wasn't particularly good at verbalising how I was feeling, and the relationship sort of broke down at that point." 
These experiences highlight the potential ramifications of knowledge gaps within mental health services. Although mental health professionals may not see themselves as harbouring queerphobic or transphobic views, a lack of knowledge about sexuality, gender, and sex characteristic diversity can compromise their ability to support rainbow clients who are exploring their identity (Lucksted, 2004). Indeed, Juno went on to say that her mental health deteriorated significantly after her counselling relationship broke down. Piper made a similar comment, reflecting that she did not think her support workers were homophobic, but they did not seem to understand the nature of sexuality; "they were straight, and they were like, if you were gay you'd be born gay and you'd know since you were a kid." Although Piper emphasised that the support workers were well-intentioned, she also noted that if she had received support in her sexuality journey earlier on she may have recovered more quickly than she did.

\section{Issues faced by groups within the rainbow community}

When discussing the need for mental health professionals to increase their knowledge about sexuality, gender, and sex characteristic diversity participants pointed out that mental health professionals must recognise the diverse nature of rainbow people and have some knowledge about the issues facing groups within rainbow communities (e.g. bisexual people, trans people, takatāpui people, etc). Participants noted that when mental health professionals lacked knowledge about the realities of their daily lives, this limited what could be discussed in therapy, as participants felt they would have to give extensive background in order for the conversation to go ahead. Lily, for example, questioned why she found it so difficult to discuss the everyday issues she encounters in her life, noting that "you can always say who or what you are, or what your situation is, but as soon as I want to start addressing some kind of actual issues in the doing of my life it becomes difficult to."

Participants mentioned a number of issues specific to groups within the rainbow community they would have liked to discuss in therapy but had refrained from doing so due to a perceived lack of knowledge on the part of mental health professionals. Groupspecific issues included the difficulties that come with dating as a trans person (particularly balancing the desire to date with concerns about safety), navigating bisexual erasure and biphobia as a bisexual person, the desire to challenge toxic masculinity as a queer male, and discussing non-monogamous relationships in contexts 
where monogamy is the norm. Participants also talked about intersecting identities, such as their identity as takatāpui:

Kit: When I heard the word takatapui that really resonated with me, because a lot of people [I spent time with] were Māori and they would look to me as kind of authority within our group on Māori things. And so if they (...) accept me being Māori also if I identify as takatāpui then they must inextricably accept me as being of the rainbow as well.

During her interview, Kit drew on Kerekere's (2017a) work to explain how empowering it was to find a word that captured the ways in which her identity as Māori and her identity as queer are inextricably linked. For Kit, her sexuality is connected to wairua "it's about connecting with different energies, however they present, and so not always a male, not always a female." Kit noted that often her mental health professionals had lacked a full understanding of Māoritanga, but that this was not always necessary for her to discuss wairua in session. Rather, it was important that mental health professionals were able to use her vocabulary, rather than their own. She noted that one professional checked in around what words she wanted to use: "and I was like 'oh! Wairua' and then the second he started saying wairua all the time I was like, oh, this is perfect." She explained that his use of wairua in session helped her to engage and to feel safe in the space.

\section{The relationship between identity, trauma, and mental health}

Across the interviews, several participants reported that their mental health professionals had seemed to search for a cause for their identity. Often, this involved connecting participants' identities with past trauma. Bonnie recalled that she had once shared that she was queer with a new therapist, and in response, "he basically was like 'oh, so you've been sexually abused before?' (...) I was like 'are you fucking kidding me? Am I dreaming?'” Others shared similar stories:

Henry: I've had mental health professionals, you know, ask me if my identity, you know, would be the same if I wasn't abused.

Felix: I have an incredibly bad relationship with my father and he abused me for quite a few years, and at one point [professionals] were kind of like, "oh 
have you ever thought about if you may be trying to be a better man than he was and the only reason you're trans is because you were abused by him?"

The fact that mental health professionals continue to connect rainbow identities with childhood trauma is incredibly concerning, not only because this is likely to extinguish any chance of building a good therapeutic relationship, but because of the distress it causes clients when mental health professionals (who are in positions of power) reify harmful misconceptions that rainbow identities originate from harm, rather than being part of usual human diversity. It must be noted that it is also, in some ways, unsurprising that this practice continues, given that the connection between rainbow identities and childhood abuse is frequently made in research literature (see, for example, Balsam, Rothblum, \& Beauchaine, 2005; Hughes, Haas, Razzano, Cassidy, \& Matthews, 2000; Lechner, Vogel, Garcia-Shelton, Leichter, \& Steibel, 1993; Morris \& Balsam, 2003), and mental health professionals are actively encouraged to turn to research literature to inform their practice (Sullivan, 2007).

As well as professionals making connections between past trauma and identity, participants described instances of mental health professionals connecting gender dysphoria to past drug use or diagnoses such as Asperger's syndrome, borderline personality disorder (BPD), and psychotic disorders. Lily, who had a diagnosis of BPD, noted that her psychologist seemed to understand her trans identity as an "identity issue" related to her BPD, and questioned whether building a secure set of friendships would "then see if that washes [my trans identity] away." Similarly, Star, who received a diagnosis of schizoaffective disorder (bipolar type) shared:

Star: When you end up with a disorder that's very associated with being impulsive and making impulsive decisions, then there's a lot of like speculation about whether it's linked to manifestations of identity.

Star went on to note that, even if her gender expression was connected to her other mental health diagnoses, that did not make her "less gay or less trans." The idea that there are 'real' and 'false' trans identities is prevalent within literature about the intersection of gender and mental disorder; Baltieri and De Andrade (2009), for example, argue that when a client is seeking gender-affirming healthcare, "underlying psychotic disorder must be excluded prior to recommending hormonal or surgical intervention" (p. 1185). Star (together with several other participants) challenged this 
position, arguing that questioning gender and experiencing mental health difficulties such as psychosis are not mutually exclusive.

For many participants, it seemed that keeping what is identified in research as 'minority stress theory' (e.g. Meyer, 2003) in mind was important in challenging internalised homophobia and transphobia, as well as explicit discrimination on the part of mental health professionals:

Naomi: I'm not mentally unwell because I'm queer, I'm mentally unwell because of how society treats me, because I'm queer.

Taylor: There is definitely a relationship between my mental health and my sexuality but I think it's like a much more nuanced relationship than " $A$ and $B$ go together".

Martin: I think as minorities, queer trans people, we are particularly vulnerable. We're overrepresented in all of the negative mental health statistics, all the addiction statistics, homelessness, unemployment, everything, and I think often it's not about being queer yourself, I'm fine with being queer and trans, but it's everybody else outside of that.

Here, Naomi, Taylor, and Martin explain that the relationship between their identity and mental health is indirect; as rainbow community members they are vulnerable to stigma and discrimination, and the stress associated with this negatively impacts on their mental health. By taking on this non-pathologising perspective participants were able to account for their own mental health difficulties, and for the high rates of mental health difficulties in their wider rainbow communities, while also holding the idea that their rainbow identities were a source of strength and pride.

\section{Educating professionals}

When mental health professionals lacked knowledge in issues relating to sexuality, gender, and sex characteristic diversity, or about the intersection of identity and mental health, participants commonly shared that they ended up educating their professionals in session. They noted that, over time, they felt worn out by this process; "half of the time it's walking everyone through, you know, babies first, gender expression and identity, and it's exhausting" (Willow). Participants also emphasised 
how unjust it felt that they needed to provide education in order to move on with therapy:

Hugo: I don't want to educate you, I shouldn't have to, as well.

Theo: You can't be bothered explaining it sometimes cause you're always explaining it to these other people. I'd think they'd know, I'd like to think that, but they just don't, and it's like, you're supposed to be the one kind of helping me and I'm helping you!

Star: I hate the experience of like, I don't know how much you know about this, and I'm going to have to explain it to you, and I'm going to have to answer your weird questions, as though like you're like an acquaintance I've just met and not the person who's giving me care. That's a weird one.

Here, Hugo, Theo, and Star stress that it is not only unreasonable that they are expected to spend time informing the person who is supposed to be giving them care, but that they do not enjoy doing so; it feels like a chore or hindrance. Theo and Star note that providing education is part of their day-to-day lives, and they would like to think that healthcare services are spaces where they get a reprieve from having to explain their identity to others.

Participants described varying reactions to their education of mental health professionals. While some mental health professionals were grateful to learn more about sexuality, gender, and sex characteristic diversity, others appeared disinterested. Taylor, for example, had described the meaning of nonbinary gender to a psychiatrist "I like, kind of tried to explain it to her and she was kind of just like, oh... whatever. She clearly didn't think that much of it." Charlie described similar experiences:

Charlie: I've tried to explain about being nonbinary but it's felt like people were nodding along or a lot of feeling that people just thought it was kind of like a fad, not really real, so it was like, explaining the terms but not getting a lot of response back.

Some participants noted that when mental health professionals seemed to understand particular identities as 'fads', or 'not really real', this made them worry about or question their own identity. This reflects previous literature in which rainbow clients reported their mental health professionals had denied the reality of their sexual 
orientation or dismissed it as a fad or phase (McFarlane, 1998; Nystrom, 1997; Platzer, 2006). Interviewees also reported that educating professionals took up a considerable amount of time in the session, limiting the time available to discuss what they had presented to therapy to talk through.

\section{Knowledgeable professionals}

While conversation around mental health professionals' knowledge focussed predominantly on gaps in knowledge, some participants reported that they had worked with mental health professionals who had taken the time to educate themselves about rainbow issues:

Bonnie: With my current therapist it's never felt like, 'you need to tell me about this, I don't understand' (...) she'll go and research outside of our meetings so she knows what she's talking about, and that's really fucking comforting, cause she actually seems to give a shit (...) the fact that she's kind of going out of her way to make sure she's using correct language and make sure she's got her research all done and stuff, it's just nice.

Here, Bonnie explained that when her therapist identifies a knowledge gap she carries out research after the therapy session, rather than asking Bonnie to talk her through it in session. Importantly, Bonnie did not seem to expect that her mental health professional would be equipped with all the knowledge she needed when they first met; she later noted that because the queer community is so diverse, she had had to talk her therapist through her "niche issues," but because this felt like a collaborative exercise, she was happy to do so. Lesley shared a similar story about her current GP:

Lesley: It's shifted from kind of like, you know, having to do that mahi and walk them through to my current GP who does her own research, so we can have conversations back and forth and just mention a couple of things she doesn't know, that sort of thing, which is nice cause she's genuinely interested.

Lesley explains that in the past she carried the workload of educating her professionals, whereas now her GP carries this workload herself. For Lesley and Bonnie, when health professionals seek education, this demonstrates genuine care and interest in their client's lives. This reflects literature showing the importance of therapist-client collaboration in 
developing a strong therapeutic relationship, which is in turn a reliable predictor of a positive therapeutic outcome (Lambert \& Barley, 2001; McCabe \& Priebe, 2004).

\section{Summary}

Throughout the interviews, the most common criticism of mental health service provision for rainbow community members was mental health professionals' lack of knowledge about sexuality, gender, and sex characteristic diversity, and the complex interplay between identity and wellbeing. Participants commonly reported that they had felt a need to educate their mental health professional in order to move forward in therapy and emphasised the exhausting and unjust nature of these interactions. Some participants had worked with mental health professionals who had explicitly sought education about rainbow issues and noted that this significantly improved the quality of their therapeutic relationship.

\section{Theme Five: Clinical Skills}

Although discussions around the quality of the support received within mental health settings primarily centred around professionals' knowledge of sexuality, gender, and sex characteristic diversity, participants emphasised that knowledge of rainbow issues is not sufficient for supporting rainbow community members; mental health professionals must also draw on their basic clinical skills of reflective listening, empathy, and validation. Participants' perceptions of their professionals' clinical skill varied. While some participants had found their mental health professionals respectful and affirming, others felt their professionals had not listened to their needs in therapy. Participants who described negative mental health support experiences commonly felt that their mental health professional had focussed on the wrong topics in therapy.

\section{The wrong focus}

Participants who reported that their professionals had focussed on the wrong topics in therapy fell into two groups. The first was those who felt their mental health professional had focussed on their identity when the participant did not want to. Hugo, a trans man, shared that he had recently seen a psychologist to talk about feeling down after moving to a new city. During the session, his psychologist had asked about his hormone levels and whether he felt comfortable about his gender: 
Hugo: They kept on making the conversation go towards a place where I didn't want it to go which was gender identity, when I was like "I'm okay within my gender, I'm okay within myself, please stop asking me" and then she got all like "oh, but you know what if it might be important? It might be, you know, relevant to this conversation?" And I just said to her "it's not".

Hugo perceived his psychologist's focus on identity as reflecting an assumption that, if Hugo was distressed, this distress must be connected to his gender identity. While it is impossible to know the psychologist's reasoning for asking Hugo about hormone levels and gender, ${ }^{42}$ the fact that she repeatedly returned to this topic against Hugo's wishes seems to indicate that the psychologist felt she knew better than Hugo about why he was distressed. This was incredibly detrimental to the therapeutic relationship - the quality of which is a strong predictor of success in therapy (Lambert \& Barley, 2001; McCabe $\&$ Priebe, 2004).

Other participants told similar stories of their mental health professionals focussing on their identity when they wanted to discuss other topics:

Lily: I saw a social worker who said while I wasn't really there for gender stuff, to them I was? It kept being brought up as possible explanations for things, like relationship problems were apparently explainable through being trans and having trans-related stuff.

Kit: When I have disclosed my sexuality then there's been some more directing, directive sort of questions, or posing of scenarios that we might talk about, or bringing things back around to that, as opposed to reflecting, "this is what I'm hearing you're saying?"

Martin: I told her that I was trans, and she wanted to focus on that, and I was like not here for that, here for a break up (...) she just kept focussing on it and I

\footnotetext{
${ }^{42}$ It is, for example, possible that the psychologist thought there was relationship between Hugo's hormone levels and mood. Even so, it is worth noting that psychologists typically do not receive training about hormone levels, so any questioning around this would be best left to an endocrinologist. Hugo commented that even if he had told the psychologist what his hormone levels were, she was unlikely to have known whether they were within a normal range.
} 
wasn't getting what I needed to out of the session, so it wasn't worth my time, and I felt quite frustrated that she wasn't hearing.

The fact that participants shared that their mental health professionals began to direct conversations after their clients mentioned their rainbow identity (rather than following the client's lead about what to discuss) seems to directly conflict with core clinical skills of validation and reflective listening. As well as being professionally incompetent, this can be understood as a continuation of the violence rainbow people face on a wider scale; for many participants, when conversation was steered toward their identity, or when identity was used to account for their mental health difficulties, this closely reflected the questioning and invalidation they had experienced throughout their entire lives.

Some participants reflected that mental health professionals may raise identity as a topic of discussion if they had a reason to believe that this contributed to the client's presenting difficulties. Kit, for example, noted that "I understand from theory sides of things, why we might go back to some of those things." She went on to say, however, that if the client does not want to discuss their identity in session, this should override any professional knowledge - "the authority to do that should lay with me, you could offer it as a choice (...) but don't tell me to talk about it or bring it back up." This highlights that, sometimes, core skills and competencies may appear to be at odds with one another in therapeutic practice, and it is the task of the mental health professional to balance these in the best interest of their client. For example, mental health professionals may understand that minority stress can have a significant impact on rainbow peoples' mental health but be asked in session by their client not to discuss their rainbow identity. While avoiding the topic of identity may feel like a potential barrier to moving forward in therapy, Kit demonstrates that it would be inappropriate to insist that identity is discussed, as this communicates disrespect of a client's wishes.

The second group of participants who were dissatisfied by the focus of their therapy were those who wanted to discuss identity, but felt their professionals steered them away from the topic:

Penny: The psychiatrist that I was seeing was not helpful, not understanding and basically was like okay, the issue is depression, and that's basically all they 
let me talk about. So if I tried to talk about gender issues or stuff, they basically just shut down the conversation.

Penny explained that she had been hospitalised after a suicide attempt and saw the psychiatrist while in the hospital. Her inability to access gender-affirming healthcare had a significant impact on her mental health, and Penny shared that she urgently needed to discuss the ways in which her dysphoria was impacting her depression and anxiety. Other participants shared similar stories:

Juno: I sort of said to [my counsellor] "well, I'm intersex, and there's a community, and I want to join this community cause there's other people like me who are experiencing the same thing, and it's nice to connect", and she basically said to me "well, you identify as female don't you", and I said yes, and she said "you've had surgery, and you're okay", and I said yes, and she said "you're not sick", and I said no, and she said "well, why are you making such a big deal about this?"

As noted above when discussing Hugo's experience (see pages 107-108), it is impossible to know why Penny and Juno's mental health professionals were unwilling to discuss their identity, even when it was specifically raised as important. It may be that the professionals did not see identity as relevant to their clients' mental health difficulties, or that the professionals felt it was best to avoid the topic due to their own lack of knowledge about sexuality, gender, and sex characteristic diversity. Regardless of the reason, this tendency to ignore clients' identity after it was raised had the same impact as mental health professionals' overemphasis on their clients' identity when it is not relevant - it made participants feel invalidated and unheard, and for many, it exacerbated their distress.

Participants' talk about overemphasising or underemphasising the importance of identity suggested that they were eager to take responsibility for deciding on the focus of discussion in therapy. Participants stressed the importance of mental health professionals checking in with their rainbow clients about whether they wanted to discuss sexuality, gender, and sex characteristic diversity, or how they saw the relationship between their identity and their mental health. This way, professionals can use their client's responses to guide the conversation: 
Willow: Maybe the client doesn't want to talk about [identity] at all, but if you bring it up, that's now there for the client to think about, but maybe they're not ready to take that into account yet, maybe they've got to take a bit more time before it sinks into them. Either way, it's just respecting their wishes, which is not hard.

Charlie: I think as long as the counsellors and health professionals know how to not be aggressive about it and when to say, like if somebody's like "hey I want to talk about my dad" or "I want to talk about depression" you just kind of focus on that, and if the other person wants to go into their sexuality or gender then go into it, but it is something that I think is important to base around the person seeking help.

Willow and Charlie describe a client-centred approach, where the focus of therapy or topic of discussion is decided in response to clients' needs. Importantly, this does not prohibit mental health professionals from raising topics their clients have not mentioned or making connections for their clients and checking in on whether these make sense to them. In their discussion of 'clients as experts', Anderson and Goolishian (2009) describe the therapeutic conversation as "a mechanism through which the therapist and the client participate in the co-development of new meanings, new realities, and new narratives" (p. 29, emphasis added). As such, most therapists will provide some degree of direction in conversation with their clients. It is also worth noting that some clients come to therapy with little idea of where to start or what to discuss, so therapists may need to take on a more directive role in some cases to move the therapeutic conversation forward. Overall, participants' talk about the focus of therapy emphasised the importance of sensitivity when directing these conversations, and respecting clients' readiness to discuss (or not discuss) sex, sexuality, and gender identity.

\section{Normalising and validating identity}

In conversation around what participants need in mental health settings, normalising and validating identity were commonly described as essential for effective therapy: 
Willow: I think that a really big important thing for mental health professionals is just straight up validation, you're okay, that's fine, like, there's so much that puts gender and sexuality as abnormal.

Andie: If you're going to be seeking counselling you need to be talking to understanding people who are gonna validate you and convince you that you're not a bad person or making things up.

In these extracts, Willow and Andie emphasise the importance of mental health professionals taking an explicitly rainbow-affirmative stance in work with queer, trans, and intersex clients. They frame affirmative practice in direct opposition to pathologising understandings of rainbow identities. Andie's use of "convince" suggests that rainbow community members may present to mental health professionals with internalised queerphobic and transphobic beliefs. Puckett and Levitt (2015) posit that such beliefs develop within contexts of persistent invalidation and emphasise the importance of clinicians framing conversations about internalised stigma within the context of societal processes. They recommend that mental health professionals explore with clients where beliefs about rainbow identities (including those of sexuality and gender diversity as "abnormal" or "bad") may have come from and normalise internalised stigma as something that many rainbow people experience.

Several participants described positive experiences with professionals who they felt took them seriously, were respectful, and created a safe space in which they could talk freely about what was going on for them. Participants emphasised the importance of active listening; Juno noted that "I've told [my psychologist] stuff like four months ago, which I thought was trivial and she remembers it word for word and yeah she does take some notes, but the notes aren't sitting next to her in the consultation." Lily, a trans woman, recalled the first time her counsellor had raised the topic of gender in session, by complimenting Lily's makeup. At the time Lily was in her mid-teens and identified as a queer man. She noted that "I always felt like it started off being not a problem, it was about just, we're gonna talk about it cause you look good!"

While Lily described her relationship with her counsellor as respectful and collaborative, other participants pointed out that the act of validating a client's identity can be a delicate balance. They noted that the "straight up" validation that Willow described on the previous page ("you're okay, that's fine") could be invaluable for a 
client presenting to a mental health professional early in their coming out journey. For others, however, a mental health professional going out of their way to express that being part of the rainbow community is okay could feel condescending or heavy handed:

Keith: There is a scale where people can either be really intolerant or over tolerant to the point where it's a bit creepy and patronising.

Piper: I think it is nice to have someone explicitly say that they're okay with treating people that are - I don't know. I mean that's not what you'd say, you wouldn't be like "oh yeah, I'm fine with the gays".

Keith and Piper's comments demonstrate that the way mental health professionals provide validation must be carefully matched to the client. For someone who has never come out before, is nervous about a discriminatory response, or is struggling with internalised stigma, mental health professionals may need to provide very explicit validation (like that described by Willow and Andie on page 111). For those who are untroubled by coming out, however, the absence of a surprised look or invasive question may be all the validation that is needed. The next question, of course, is how are mental health professionals to know which response is appropriate? This seems to be where clinical skill (and, likely, clinical experience) comes in; mental health professionals can tell a lot from a person's demeanour when they come out (for example, are they crying? Do they look nervous? Was this mentioned casually, along with the fact that they have a cat and work as an accountant?). Mental health professionals can also check in with clients about what it meant for them to share and discuss identity.

\section{Summary}

In discussions of what makes a positive therapy experience, participants explained that both knowledge of rainbow issues and the use of basic clinical skills are essential for supporting rainbow community members. Several participants explained that their professionals had either overemphasised or underemphasised their identity during therapy and noted that mental health professionals should check with clients about what they want to discuss in session and respect their wishes regarding this. Participants who reported positive experiences in therapy described their mental health 
professionals as respectful, affirming, and validating. It is important to note, however, that professionals may have to apply their clinical skills flexibly, as what feels validating for one client may feel patronising for another.

\section{Theme Six: Rainbow Champions}

The themes I have discussed thus far suggest that participants in the current study had mixed experiences of accessing mental health support. Though some participants reported that they found their mental health professionals knowledgeable and supportive, conversation was dominated by talk about the difficulties of coming out in mental health settings, and the ways in which participants felt mental health professionals needed to improve their knowledge of sexuality, gender, and sex characteristic diversity in work with rainbow clients.

After discussing participants' experiences of accessing mental health support I ended interviews by asking them to share their reasons for taking part in the study, and whether they had any feedback for me to improve or change future interviews. I asked these questions because many participants shared difficult experiences over the course of our conversation, and I wanted to close the interviews with some easier content rather than end abruptly. I also asked these questions for my own interest, in the hopes the responses would help with future recruitment and improving my interviewing skills. I did not anticipate that this part of the interview would make its way into my analysis, however the care participants had for others in their community was striking when I asked about their reasons for participating in the study. The costs of this care further emphasise the need for accessible mental health support and suggests that those outside of rainbow communities should engage in rainbow advocacy and activism; work which is, currently, left primarily to rainbow people.

\section{Care for community}

Despite the hardship that participants had faced, both in society and in mental health settings, participants expressed a deep care for other rainbow people, and a desire to give back to their communities. Participants commonly reported that they took part in the current study in the hopes of making positive change for the rainbow young people that followed after them: 
Cleo: If I've been through everything that I've been through, I need to make something out of it, and if I can make a change, or I can help someone who used to be like the way I was, I'm going to do it, I'm going to everything that I can, I'm going to make the most of going through this journey.

Charlie: I want the experience to be better than it was for me. My life could have been a lot better if I'd had services that I felt safe in earlier, it could have been so much better, and I want it to be good for the generation coming after me because they deserve that.

Here, Cleo and Charlie acknowledge the difficulties they had in navigating mental health services as rainbow community members and imagine a future in which rainbow young people can feel safe and supported when accessing mental health care. Like Cleo and Charlie, most participants saw taking part in research as a tangible way of creating positive shifts for the rainbow community. Bats noted that "this is the stuff that actually gets things changed" while Theo stated, "I feel like this research is gonna be really impactful for future." Some participants also described personal benefits to taking part in the research, sharing that it was "heartening" to hear that someone outside of the community wanted to improve the quality of mental health support for rainbow people, or that it was helpful to talk through some of their past experiences; Juno shared that “it's a healing process as well, it's not just you receiving this information for your research, discussing it justifies my identity, which was really powerful, so thank you for making that space."

\section{The costs of care}

I greatly admired the empathy and care that participants had for others in their community and found it inspiring that they were taking time to be interviewed for a study in the hopes that it would create change for other rainbow people. It did seem, however, that this care often came at a cost. Participants who worked in rainbow or activist communities noted that they often found it difficult to care for themselves when they felt a responsibility to care for others. Wallis shared that "it's hard, doing this work," while Alex noted that supporting others is a form of emotional labour, and stressed the "need to shift this burden" from peers to professionals who are paid to provide this support. 
Alex's point about 'shifting the burden' relates back to the structural factors identified in Theme 1. In the context of an underfunded and overstretched mental health system, it seems that the responsibility for providing care can shift from paid professionals onto community members, who are often themselves in need of care. This has potential to create a downward spiral, in which rainbow people have high mental health needs but often feel they cannot rely on the mental health system to meet those needs, so they take on a responsibility to support each other. This puts them under further stress, in turn leading to even greater mental health disparities.

Participants' talk about feeling a responsibility to care for one another highlights the need for those outside the rainbow community to confront homophobia, biphobia, transphobia, and prejudice against intersex people, to ensure that this responsibility does not fall solely on the shoulders of rainbow people. Here, it must be acknowledged that allyship can be complex, as majority members must attempt to confront prejudice without speaking on behalf of rainbow community members (Happell \& Scholz, 2018). It is, however, arguably more important for straight and cis people to support rainbow communities, and potentially make mistakes along the way, than leave this work entirely to the rainbow community.

\section{Summary}

In the face of significant adversity, both in their day-to-day lives and in mental health settings, participants shared a strong desire to give back to their communities, who they often felt had supported them when formal systems of support had failed. Taking part in the current study was one way in which participants felt they were able to give back, though many also did so through other work and activism. As a researcher this generosity was touching, but also concerning, as it has the potential to shift the burden of providing care from paid professionals onto the shoulders of those who need care themselves.

\section{General Discussion}

Taken together, participants' talk about their experiences accessing mental healthcare painted a picture of a system struggling to meet rainbow peoples' high levels of need. When participants did access support, their experiences were mixed; some described professionals who they found affirming, validating, and knowledgeable, while 
others described a widespread lack of knowledge and, at times, a lack of basic clinical skill. Participants highlighted that therapy takes place within a broader heteronormative and cisnormative context. This context constrains the ways in which mental health professionals and clients can discuss sexuality, gender, and sex characteristic diversity, as well as the intersection of identity and mental health. When rainbow people are unable to access the support they need through New Zealand's mental health system this can shift responsibility for providing care on to rainbow communities, which creates increased stress for those often in need of support themselves.

\section{Links with Wider Literature}

Participants' overall mixed experiences of mental health support reflected a wider body of literature showing varied findings in relation to rainbow therapy experiences (King et al., 2007; White \& Fontenot, 2019). It was unsurprising that there is no one story when it comes to rainbow therapy experiences, as the nature of a therapy experience is likely dependent on a wide range of factors, including client characteristics, professional characteristics, as well as where and when mental health support was accessed. It was interesting to note that, similar to previous research studies (e.g. Bowers et al., 2005; Lucksted, 2004; Nystrom, 1997; Page, 2004; Poteat et al., 2013), talk was dominated by negative experiences of accessing mental health support. Prior to conducting the next stage of the research, I was interested to explore whether survey participants would also focus predominantly on negative experiences, or whether they would present a more positive view of their experiences accessing mental health support. I was also eager to explore whether survey participants' experiences differed according to the type of professional they sought support from (e.g. psychologists, counsellors, psychiatrists), as well as the participants' demographic characteristics (e.g. gender, age, region, ethnicity), which could not be examined in the interview study.

My interview participants described several experiences in mental health settings that have also been described previously, such as being met with surprise and discomfort after coming out (Eady et al., 2011; Evans \& Barker, 2010; McCullough et al., 2017; Saulnier, 2002), feeling a need to educate their professionals (Bauer et al., 2009; Corliss et al., 2007; Evans \& Barker, 2010; Grove, 2009; Kidd et al., 2011; Lucksted, 2004; McCann \& Sharek, 2014; McIntyre et al., 2011), not being given space to discuss their identity (Daley, 2010; Kidd et al., 2011), or having their identity made 
the focus where it was not relevant (Israel et al., 2008). Participants in the current study commonly shared that their mental health professionals had made assumptions about how they identified their sexuality or gender, which is widely reported in extant literature (Eady et al., 2011; Hunt et al., 2006; Liddle, 1996).

The negative experiences described by participants in the current study more closely reflect past research describing subtle discrimination and microaggressions (Daley, 2010; Kidd et al., 2011; Shelton \& Delgado-Romero, 2011) and a general lack of knowledge about rainbow needs and experiences (Hunt et al., 2006; Lucksted, 2004; McCullough et al., 2017; Poteat et al., 2013; Shelton \& Delgado-Romero, 2011), rather than instances of overt discrimination and pathologisation (Liddle, 1996; Lucksted, 2004; Nystrom, 1997; Willging et al., 2006). This supports the hypothesis that, overall, mental health professionals have moved along the 'continuum of negative experiences' (see page 26) over time. The subtle discrimination participants describe could also reflect a wider trend in New Zealand for prejudice to be expressed indirectly, through bias or acts of microaggression (Farvid, 2018), rather than through overt acts of violence.

While the lack of overt discrimination and pathologisation described in the current study is encouraging, as it reflects progress towards a more rainbow-affirmative stance in the field of mental healthcare, it is also important to acknowledge that more subtle forms of discrimination can be just as damaging to the therapeutic relationship as overt discrimination and are particularly difficult to challenge (Shelton \& DelgadoRomero, 2011). Overall, the negative experiences participants described indicates that there is still a great deal of work to be done to improve the experiences of rainbow people in therapy.

\section{Silence around identity}

The 'silence around identity' reported across interviews was consistent with findings from Semp's (2006) research on the experiences of MSM services users and queer staff within New Zealand's public mental health services. Both clients and staff in Semp's study reported that mental health professionals do not usually ask clients about their sexual orientation, with staff noting that they often use 'clinical judgement' to decide whether to initiate these conversations with clients. Semp posited that heteronormative discourses limit the ability for mental health professionals to 
acknowledge non-heterosexuality; staff in his study expressed concerns that initiating conversations about sexuality may threaten or harm their clients in some way. In turn, this silence around sexuality reinforces discourses of heterosexuality as normal and natural.

Semp (2006) recommends that mental health professionals 'start queer conversations' by asking all clients about sexuality and homonegative trauma. Research conducted by Canada's Centre for Addiction and Mental Health (CAMH; Barbara \& Doctor, 2007) also suggested that initiating conversations about gender and sexuality disrupts a heteronormative and cisnormative silence and is necessary for effective treatment and counselling. In contrast to Semp's recommendations (Semp advocates for open questions such as "Have you ever had any concerns about your sexuality?") $\mathrm{CAMH}$ asserts that it is important to determine the gender identity and sexual orientation of all clients (they provide interview guides and surveys to support mental health professionals with 'asking the right questions'; Barbara \& Doctor, 2007).

When I asked participants whether it would be helpful for mental health professionals to ask questions around sexual orientation and gender identity, however, there was a wide range of responses, with some saying that this would feel validating and would open up the conversation, and others noting that this could feel confronting and unsafe. As such, the current study suggests that open questions, as Semp (2006) recommends, may be more appropriate than direct questions about identity, as Canada's CAMH (Barbara \& Doctor, 2007) recommend. This analysis also elaborates on subtle ways mental health services and professionals can open up these conversations, by displaying visual cues, using expansive language, checking and sharing pronouns, and having all gender bathrooms and inclusive forms.

\section{Structural barriers to accessing support}

In the midst of dozens of media reports investigating the current state of Aotearoa's mental health system, ${ }^{43}$ very few past studies examine structural barriers to

\footnotetext{
${ }^{43}$ See, for example, Arthur-Worsop (2016), Broughton 2016; 2017), Brown (2017), Campbell (2017), Cann (2017), Clark (2017), Clayton (2017), Cowlishaw (2017), Genter (2017), Goodwin (2017), Hutton (2017), King (2017), MacDonald (2016), Martin (2017), McAllen (2016; 2017), Meier (2017), Robson (2017), Wiggins (2017), Wright (2016).
} 
accessing mental health services in New Zealand. Structural barriers were documented in Adams and colleagues' (2012) needs assessment on the mental health promotion and suicide prevention needs of rainbow New Zealanders. Specifically, respondents reported that a lack of publicly funded mental health services and the high cost of privately funded support formed significant barriers to access. This, in turn, prolonged respondents' distress and "might lead to a disproportionate burden on health resources later on" (p. 49). Adams and colleagues' (2012) discussion of structural barriers to accessing support closely reflects those described in the current study, where participants reported increased distress and, for some, increased risk of suicidality.

Although academic literature sheds little light as to whether the experiences of these 34 participants are typical of those who attempt to access mental health support in Aotearoa, the extensive media coverage of New Zealand's mental health sector provides evidence to suggest that the structural barriers encountered by participants were common in Aotearoa at the time they were interviewed (and subsequently). Media articles from this time include numerous accounts of New Zealanders waiting for months to access mental health services (e.g. Broughton, 2016; Goodwin, 2017; Martin, 2017), or being stuck in a 'grey zone' where they were not deemed unwell enough to get help (e.g. Robson, 2017; Wiggins, 2017). Participants' stories also closely reflect those presented in the People's Mental Health Report (Elliott, 2017); a crowdfunded, story-based review of New Zealand's mental health services; ${ }^{44}$ over a third of stories submitted to the People's Mental Health Report were about difficulties in accessing mental health services, including lengthy waiting times, and failing to meet criteria for publicly funded support. A number of contributors to the Report felt that they had to be in crisis to access services and emphasised the importance of early intervention. Finally, the structural barriers described by participants in the current study were consistent with those subsequently described in the Report of the Government Inquiry into Mental Health and Addiction (2018), which described "a system under stress characterised by

\footnotetext{
${ }^{44}$ The People's Mental Health Report was an initiative run by ActionStation, a community campaigning organisation. The Report received widespread media coverage, and ActionStation claim this helped to create the political conditions for the subsequent Mental Health Inquiry (ActionStation, 2019).
} 
long delays, overworked staff, inadequate environments, a lack of clear information and gatekeeping rules that served as barriers" (p. 54).

\section{Implications and Future Directions}

Based on my analysis of participants' talk about accessing mental health support, I argue for two key points of intervention to inform the provision of high quality and responsive mental health care for rainbow people in New Zealand. The first is intervention to dismantle structural barriers to accessing support, which would lead to shorter waiting times and fewer rejected referrals due to not meeting the cut-off for publicly funded services. As discussed in Chapter 2 (see page 10), in the time between conducting these interviews and submitting this thesis, the New Zealand Government promised $\$ 455.1$ million to fund a new universal frontline mental health service to address structural barriers in mental health service provision (Bennett, 2019). At the time of writing service users continued to encounter lengthy waiting times and rejected referrals in New Zealand's public mental health; however, as Walters (2019) points out, "the Government's plan to change the way the country responds to mental health will take time to flow through into measurable change" (para. 1).

With regard to the limited number of explicitly rainbow-friendly services reported in the current study, as well as mental health professionals' lack of knowledge, use of clinical skill (or, sometimes, lack thereof) in discussions of sexuality, gender, and sex characteristic diversity, and widespread silence around rainbow identities, it seems that a second point of intervention has potential to improve service provision: increasing rainbow cultural competence among New Zealand's mental health professionals. Cultural competence has been defined in many different ways throughout research and practice literature, however it is commonly agreed that cultural competence means having the awareness, knowledge, and skills to support clients from a range of cultural backgrounds (New Zealand Psychologists Board, 2011; Sue \& Torino, 2005).

The most obvious way to increase mental health professionals' competence in any area is to provide resources and training. There is no publicly available information about the extent to which rainbow cultural competence is currently incorporated into New Zealand's mental health training programmes, however informal correspondence with programme directors and coordinators indicates that the provision of this material 
ranges from zero to seven hours during postgraduate qualifications, with an average of two hours. ${ }^{45}$ On top of this, no New Zealand-based professional association requires mental health professionals to engage in any further training on rainbow cultural competence to fulfil their requirements for continued professional development, meaning any rainbow cultural competence training completed by members of the existing mental health workforce is taken up voluntarily (or, potentially, at the request of individual services and organisations). In light of this, it is unsurprising that many mental health professionals may have gaps in their skills and knowledge when it comes to supporting rainbow clients.

The current study provides valuable insight into what rainbow cultural competence training for New Zealand's mental health professionals could encompass. On the basis of my analysis, mental health professionals should be encouraged to take an explicitly rainbow-affirmative stance, and to communicate this through checking clients' pronouns, using expansive language, and displaying visual signs of support in their service. Mental health professionals should also be informed about the nature of sex, sexuality, and gender, definitions of common terms, and issues faced by specific groups within rainbow communities. Other potential areas of education include the focus of therapy, the relationship between rainbow identities and mental health, and the role of therapists' self-reflection on their own beliefs about sexuality, gender, and sex characteristic diversity. I return to these ideas in Chapter 9, where I develop a resource to address mental health professionals' knowledge gaps around supporting rainbow clients.

\section{Conclusion}

The current analysis provided an in-depth exploration of the mental health support experiences of 34 rainbow young adults in Aotearoa New Zealand. Overall, participants reported mixed experiences in therapeutic settings. They encountered

\footnotetext{
45 This is based on responses from 12 of the 27 university-based mental health training programmes I contacted. One of the counsellor education programmes responded that education regarding inclusion, difference and diversity was woven into core competency skills development, with a focus on theoretical coherency applied in practice skills, rather than being taught as a separate topic. As such, this programme was not included in my calculation.
} 
widespread structural barriers to accessing mental health support, faced a silence around rainbow identities, felt that mental health professionals often lack knowledge about sexuality, gender, and sex characteristic diversity, and emphasised the importance of basic clinical skills of validation and affirmation within therapeutic settings. While talk was dominated by negative experiences in mental health settings, many participants shared that they developed a collaborative therapeutic relationship with mental health professionals who they found knowledgeable, skilled, and affirming. This analysis highlights the need for systemic change in New Zealand's mental health system to remove structural barriers to care, and calls attention to the importance of increased cultural competence training for mental health professionals.

In the next chapter, I conduct a second thematic analysis using this set of interviews, focussing on the experiences of participants who accessed gender-affirming healthcare. In Chapter 8, I use the experiences shared by participants in the current study to create a (predominantly quantitative) online survey about rainbow peoples' mental health support experiences, in order to explore whether the experiences of these 34 participants are reflected by a wider group of rainbow New Zealanders. 


\section{Chapter 7: Analysis of Interviews About Accessing Gender-Affirming Care}

In the previous chapter I presented a thematic analysis of interviews with 34 rainbow young adults about their experiences of accessing mental health support in Aotearoa. In this chapter I use thematic analysis to identify patterns of meaning across the same set of interviews, but I focus my analysis on the experiences of 13 participants who accessed gender-affirming healthcare. ${ }^{46} \mathrm{I}$ begin by providing background about the need for gender-affirming healthcare and discussing mental health professionals' role in gender-affirming healthcare provision. I then discuss three key themes I identified in participants' accounts of their experiences.

Firstly, Lack of Funding and the "Postcode Lottery"47 examines the difficulties participants reported when seeking gender-affirming healthcare due to limited funding and inconsistent healthcare provision. Secondly, Proving Gender discusses participants' experiences in mental health assessments for hormone therapy or surgery, where participants often experienced the system as designed to establish whether they were 'trans enough' or 'truly' trans. This theme also outlines participants' reflections as to why mental health assessments for gender-affirming healthcare are conducted in this manner. Finally, The Trans Narrative encompasses participants' talk about feeling pressure to present their gender in a particular way in order to gain access to the healthcare they needed. Figure 5 presents the final thematic map for this analysis, to illustrate the relationships between themes.

\footnotetext{
${ }^{46}$ Of these participants, 11 accessed gender-affirming care in Aotearoa, and two accessed care overseas. When I discuss experiences outside of New Zealand, I specify this in the analysis.

47 "Postcode lottery" was a phrase used by one participant to describe the inconsistent availability of gender-affirming healthcare across different regions of New Zealand.
} 


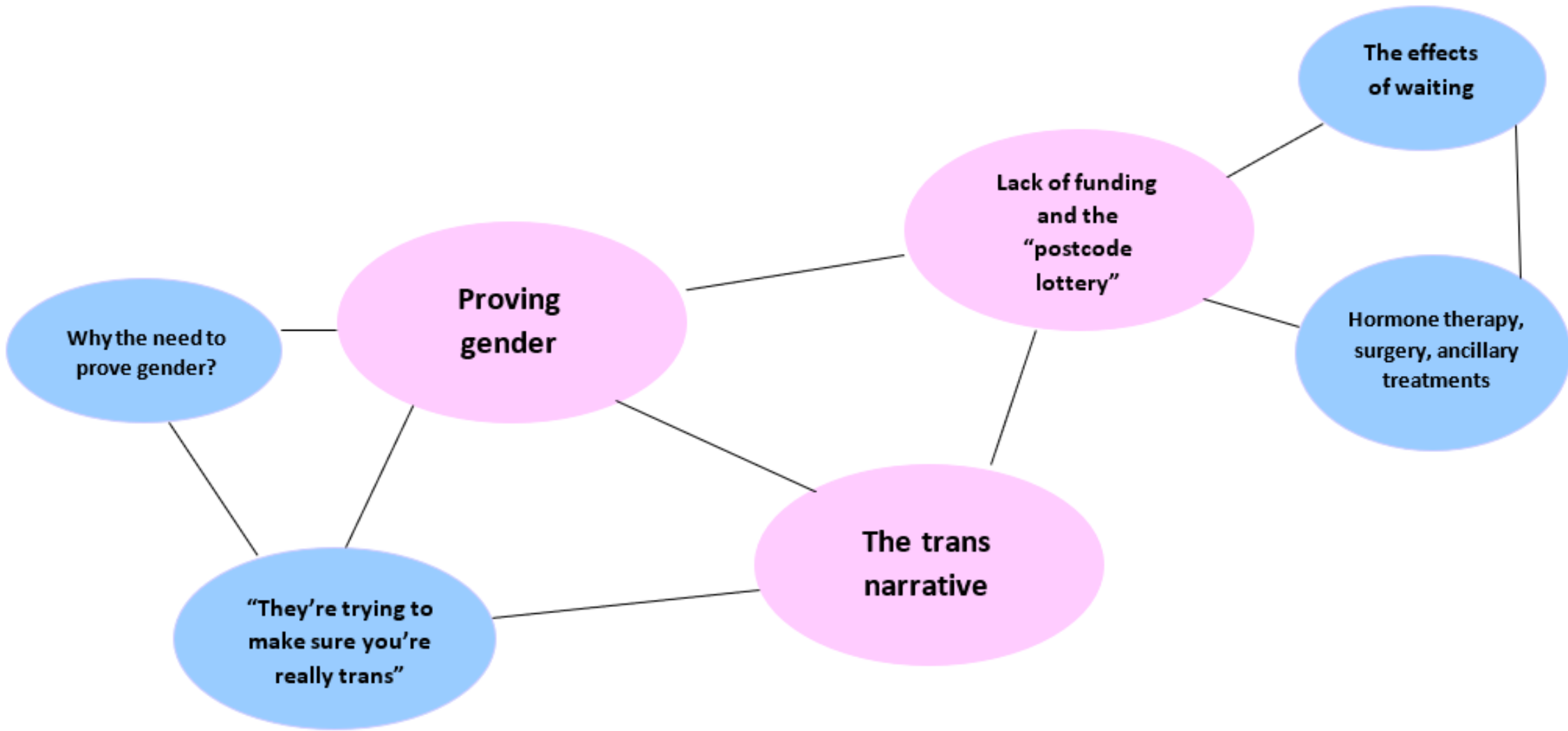

Figure 5. Final thematic map for analysis of interviews about accessing gender-affirming healthcare. 


\section{Background}

In Chapter 2 I discussed a well-established body of research documenting high rates of depression, anxiety, suicidality, and other mental health difficulties among trans community members (Budge et al., 2013; Haas et al., 2011), and showing that exposure to societal stigma increases risk for poor mental health and wellbeing (Bockting, Miner, Swinburne Romine, Hamilton, \& Coleman, 2013; White Hughto, Reisner, \& Pachankis, 2015). For many trans people, the distress associated with gender dysphoria (dissonance between one's gender and body; Gender Minorities Aotearoa, 2017) also contributes to the development of mental health difficulties (Holt, Skagerberg, \& Dunsford, 2016; Olson, Schrager, Belzer, Simons, \& Clark, 2015). As such, improving the health and wellbeing of trans community members requires challenging stigma and discrimination at a societal level, as well as providing access to mental health support and genderaffirming healthcare services for those who need it. Meta-analyses and systematic reviews of the extant research literature provide empirical support for the efficacy of gender-affirming healthcare, demonstrating that access to transition-related services decreases gender dysphoria, improves psychological symptoms, and increases quality of life (Dhejne et al., 2011; Murad et al., 2010; White Hughto \& Reisner, 2016).

Health professionals from the psy* disciplines play a key role in the provision of gender-affirming healthcare (Riggs et al., 2019). Although gender-affirming healthcare is predominantly focussed on providing physical healthcare services (e.g. hormone therapy and surgery), mental health professionals have policed trans peoples' access to gender-affirming healthcare since it was first available, due to historical understandings of trans people as mentally unwell (Vipond, 2015). Understandings of trans identities have shifted over time - the psy* disciplines now widely understand gender diversity as part of usual human variation (Drescher et al., 2012) - however, access to genderaffirming healthcare is still typically regulated by mental health professionals (Riggs et al., 2019).

To contextualise my thematic analysis and discussion I present three different models of gender-affirming healthcare provision: the pathologising model, the diagnostic model, and the informed consent model. I describe how mental health professionals' role in gender-affirming healthcare provision is understood within each model. I do this because there was considerable confusion at the time of interviewing 
(among both health professionals and service users) about mental health professionals' role in gender-affirming healthcare provision (Wi-Hongi et al., 2017). These three models provide a framework to understand participants' interactions with mental health professionals when accessing gender-affirming healthcare.

\section{The Pathologising Model}

In the pathologising model the role of the mental health professional is to ascertain that their client is truly trans and refuse gender-affirming healthcare to anyone who is not. Understandings of what it means to be 'truly trans' have changed across time, but historically involved having a binary gender identity, presenting as hyperfeminine or hypermasculine, seeking out all available medical transition services, desiring gender-affirming healthcare over a lengthy time period (usually at least two years), and feeling "born in the wrong body" (Engdahl, 2014; Riggs et al., 2019, p. 913; Vipond, 2015). The pathologising model is underpinned by the understanding that being trans reflects there is something medically wrong with the person; to be trans is an illness in need of treating, and gender-affirming healthcare is an appropriate treatment (Vipond, 2015). Versions 1-6 of the WPATH standards of care were, for the most part, consistent with the pathologising model (Berger et al., 1977, 1980, 1981, 1990; Levine et al., 1998; Meyer et al., 2001), however these guidelines are now outdated, having been replaced by guidelines that are more consistent with the diagnostic model (Coleman et al., 2012).

\section{The Diagnostic Model}

In the diagnostic model the role of the mental health professional is to ascertain that their client is experiencing gender dysphoria (the professional does not have to assess 'transness', as this is taken at the client's word), understands the risks and benefits of treatment, and is in a place where they can safely make decisions about their own medical treatment. ${ }^{48}$ According to this model being trans is not an illness to be treated. Instead, the treatment target is the distress associated with gender dysphoria

\footnotetext{
${ }^{48}$ Many mental health professionals practicing within this model also take time to discuss how the client may approach potential difficulties on their gender journey, such as safety issues, responses from friends and whānau, and navigating coming out at school or work (Bullock, 2019).
} 
(Schulz, 2018). The diagnostic model is consistent with the WPATH standards of care v7 (Coleman et al., 2012), ${ }^{49}$ which were considered international best practice guidelines at the time of interviews.

\section{The Informed Consent Model}

In the informed consent model, the role of the mental health professional is to ascertain that their client understands the risks and benefits of treatment and is able to safely make decisions about their own medical treatment (Cavanaugh, Hopwood, \& Lambert, 2016). As such, the difference between the diagnostic model and the informed consent model is that the former requires that the client is experiencing gender dysphoria (and its associated distress), while the latter does not. Mental health support is still offered as an option within the informed consent model but is not a requirement for accessing gender-affirming healthcare (Schulz, 2018). Many advocates for the informed consent model posit that trans people should have the self-determination to make decisions about their own care without having to prove to health professionals that they meet certain diagnostic criteria (Cavanaugh et al., 2016). The informed consent model is in use in some clinics, predominantly in the US (see Deutsch, 2012), but has not been widely adopted. ${ }^{50}$

\section{Standard Practice in New Zealand}

Although I outline these three models as distinct, in reality the role of mental health professionals is not so easily demarcated, and there is considerable overlap between these models. ${ }^{51}$ At the time of interviewing, the WPATH standards v7 were

\footnotetext{
${ }^{49}$ Due to the somewhat vague wording of some sections of the WPATH standards, some researchers view the WPATH standards as consistent with the informed consent model. Davy (2015), for example, states that gender dysphoria need not be present to access care according to WPATH, however I would argue otherwise, as the guidelines clearly state that the criteria for hormone therapy includes "persistent, welldocumented gender dysphoria" (Coleman et al., 2012, p. 34).

50 This may be due to an ongoing reliance on the WPATH standards, because there is a lack of research about the outcomes of clients who have used this model, or because health professionals are concerned there is an increased likelihood of clients regretting their decision if the involvement of a mental health professional is not required (Cavanaugh et al., 2016; Schulz, 2018). I return to these ideas in the general discussion section of this chapter.

${ }^{51}$ For example, health professionals generally use the DSM-V to diagnose 'gender dysphoria' (as required by the WPATH standards). Although gender dysphoria is the distress associated with dissonance between one's gender and body (Gender Minorities Aotearoa, 2017), the DSM-V section on gender
} 
used by many health professionals in New Zealand. The Ministry of Health, however, had guidelines available on their website based on the WPATH standards v6 (Ministry of Health, 2018d). ${ }^{52}$ This created confusion about what constituted standard practice in New Zealand, and meant that some health professionals' process was consistent with the diagnostic model, and some with the pathologising model. As my analysis illustrates, the model used had a profound impact on trans clients' experiences of gender-affirming healthcare.

\section{Thematic Analysis}

\section{Theme One: Lack of Funding and the "Postcode Lottery"}

Participants' discussion of barriers to accessing gender-affirming healthcare closely reflected talk around barriers to accessing general mental health support, outlined in the previous chapter (see pages 69-76). Most participants described the process as drawn out and frustrating, due to funding constraints within the public health system. ${ }^{53}$ Participants also reported that "it's kind of a postcode lottery in terms of what you can access" (Martin); the availability and accessibility of gender-affirming healthcare is often dependent on the region in which someone is trying to access care. Larger cities (such as Auckland and Wellington) were generally described as easier places to access gender-affirming healthcare than smaller cities, towns, or rural areas.

Penny stated that funding for trans health at her local DHB was so restricted, it was routine to pay for a private mental health assessment in order to access hormones, rather than going through the public health system:

dysphoria includes a number of other seemingly unrelated criteria that rely on outdated understandings of trans identities, consistent with the pathologising model: these criteria (for gender dysphoria in children) include "a strong preference for playmates of the other gender", "a strong preference for cross-gender roles in make-believe play" and "a strong preference for the toys, games, or activities stereotypically used or engaged in by the other gender” (American Psychiatric Association, 2013, p. 452).

52 These guidelines have since been updated, but readers interested in seeing the guidelines that were posted at the time of interviewing can visit http://web.archive.org/ and search the website captures from February 2018.

${ }^{53}$ As noted in Chapter 2 (see pages 12-13), some gender-affirming healthcare services are funded at a national level, and others at the DHB level (where different funding prioritisation leads to regional differences in gender-affirming healthcare availability). Participants tended not to differentiate between these two levels of funding, instead referring to a general "lack of funding". 
Penny: Most people do it privately in [local DHB] because public's useless, they admitted they don't want to know anything about it, they don't want to deal with it, cause they have no funding for it, and they have virtually no one who's knowledgeable about it. I know a range of people who've just recently done mental health assessments, it cost 500 to a grand, for some of them.

Penny, who had been involved in trans advocacy meetings with representatives from her local DHB, identified a lack of political will within her local DHB to improve access to trans healthcare, as there is no budget to provide further services and training. Rather, any change would have to be cost neutral - a near impossible requirement.

Several participants stressed the importance of increasing the funding available for trans healthcare, in order to make care more accessible:

Bats: I didn't know for the longest time that top surgery is available funded in New Zealand, it is available, it's really dependent on your damn DHB (...) I'm just angry at everything being defunded and underfunded in mental health and trans healthcare. We need more money, that's my main concern. I want things funded, I want things available, I want things streamlined, I'm so sick of hearing "oh we're working on it, we're working on it".

Here Bats argues that, although gender-affirming care is theoretically funded in New Zealand, in practice the amount of funding (and the system that facilitates funding availability) is inadequate to address the needs of trans people. Indeed, four of the 20 DHBs did not provide any gender-affirming healthcare services at the time of interviewing (Fraser, Shields, Brady, \& Wilson, 2018), meaning that those in need would have to access private care or move to another region in order to access publicly funded care. Bats expresses frustration about the lack of progress being made in this area, and that they were not made aware that the information they were given regarding the availability of top surgery was limited to their DHB. This sense of confusion about what services were publicly funded was common among trans participants, as was feeling "sick of hearing" that work was being done to improve healthcare provision.

\section{Hormone therapy}

There was notable variation in participants' accounts of accessing hormone therapy. Of the 11 participants that had accessed (or attempted to access) hormone 
therapy in New Zealand, seven had been assessed by both a mental health professional and an endocrinologist (the most common pathway in New Zealand's public health services, and a pathway consistent with the WPATH standards of care). Of those who had gone through this process, the time taken from requesting hormone therapy for the first time to receiving their first hormone dose varied from six months to over three years.

For some participants, approval for hormone therapy was delayed because professionals requested more sessions with the participant before providing a referral. For others, the process of accessing hormone therapy was delayed due to lengthy waiting times before (and between) seeing health professionals. Felix, for example, stated "I had to wait about a year to see the psychologist, because she was the only available public psychologist that catered to youth." After seeing the psychologist for two years and receiving a referral letter to endocrinology, Felix was told the endocrinologist was not taking referrals. While the service that Felix was connected with ended up funding Felix to see an endocrinologist privately, others underwent another waiting period. Willow had paid for a private mental health assessment and been referred for a publicly funded endocrinology appointment, "and I've basically just been told, 'nah, we've got a waiting list forever."' At the time of the interview, Willow was still waiting to see an endocrinologist through the public health system.

Three participants described pathways to accessing hormone therapy that they noted were somewhat unusual. These included being prescribed hormones by a GP after a private mental health assessment (with no input from an endocrinologist), being prescribed hormones by an endocrinologist with no involvement from a mental health professional and being prescribed hormones by a GP after briefly seeing an endocrinologist and a mental health professional (without receiving formal sign-off by the endocrinologist or mental health professional). In each case, the participants were unsure of whether their professionals were unaware that this was not usual practice (which participants knew based on what they had heard from others who had accessed gender-affirming healthcare) or were simply satisfied that their client was ready for hormone therapy and felt no need to send them for another assessment before approving this. Martin and Lesley explained that they generally keep quiet about their path to hormone therapy. For Lesley, this is due to a worry that her new healthcare service will 
require her to see an endocrinologist, "but it would require a new psych report, which isn't funded down here as [city] sexual health was, so that's a thing." Martin noted that "I know that I'm the exception, and so I generally don't tell my story to other trans people, because I don't want them to think that that's normal, cause it's so not."

Two participants had accessed hormone therapy while overseas. Hugo had gone through a process consistent with the diagnostic model, where he had seen both a psychiatrist and an endocrinologist. The whole process took around six months, and included four sessions with the psychiatrist, as well as one session with the endocrinologist (who Hugo described as "just there to tick a box"). Star had accessed hormones through the informed consent model, where she received her first shot of testosterone three weeks after her initial appointment:

Star: It was a very expedited process and I know that sounds scary as hell to people who aren't sure if informed consent is a good thing. But it was really really good for me because (...) it can be very frustrating when you spend a couple years psyching yourself up about whether you're actually trans. You spend a couple years psyching yourself up about whether you want to transition, or whether you're just going to keep the secret with you to the grave. And then you spend some time like psyching yourself up about how to come out to like friends and family and parents. And then, you have to go through gender therapy, and after 6 months of that you can go on hormones. And then you have to wait for all the changes to start kicking in and the whole time you're going crazy going like "I need this to happen faster", you know?

Here, Star explains that, although three weeks sounds like very little time for the process of accessing hormone therapy, she had been considering hormone therapy for several years before she presented for her first appointment. Star's experience suggests that the historical requirement for a period of "real-life experience" in the WPATH standards of care (intended to “"test' the person's resolve, the capacity to function in the preferred gender and the adequacy of social, economic, and psychological supports"; Meyer et al., 2001, p. 24) is based on faulty logic. Although real-life experience is no longer a requirement in the WPATH standards of care, Penny and Felix were both told by their endocrinologist they had to wait between one and two months before hormones were prescribed, in order to give time to "think it over." 


\section{Surgery}

While participants described accessing hormone therapy as difficult, accessing surgery was described as virtually impossible within New Zealand's public health system. Bats reported that they had requested a referral for top surgery at their youth health service - "they were like oh there's no surgeries available, so I'm like okay, I guess I'll just do nothing!” Indeed, all of the participants who had accessed top surgery had done so overseas, either through other public health systems, or by self-funding the procedure. Taylor noted that because surgery is so difficult to access in New Zealand, several of her friends had used online crowdfunding platforms, such as GoFundMe and Kickstarter, to fundraise for their top surgeries. She described it as "absurd" that her friends had to raise such vast sums of money "to remove something so useless to them."

Nykk shared that they had considered applying for publicly-funded surgery, but had decided against it:

Nykk: It was something I was going to do, and then I looked into it and realised there's no point. The trans surgeries, the average waiting time to get it is 32 years, and I was like what's the point of me doing anything? I'll be an old person by the time I get there, what's the point, I felt like there was just no point (...) you'd be more likely to win lottery basically than be able to get surgery in New Zealand.

Nykk's experience highlights a key issue with surgery waitlists in New Zealand. As noted on page 12, at the time of interviewing the waitlist for genital surgery was over 50 years long (Ministry of Health, 2018c) meaning that, like Nykk, many trans people in need of surgery chose not to go on the waitlist. Wi-Hongi and colleagues (2017) refer to this as a Catch-22; doctors are discouraged from putting their patients on such an unethically lengthy waiting list, but the Ministry of Health uses the list to guide funding decisions. As such, the length of the waiting list does not capture the true demand for gender-affirming surgeries and improving funding for trans healthcare "remains a political non-priority" (p. 164). ${ }^{54}$

\footnotetext{
${ }^{54}$ This description of gender-affirming healthcare as a "political non-priority" was accurate at the time of interviewing, however the election of a left-wing coalition Government in 2017 did result in an additional
} 


\section{Ancillary treatments}

Along with conversations around hormone therapy and surgery, participants discussed the difficulties of accessing ancillary treatments, such as laser hair removal. Nala noted that "still having facial hair is just the worst," while Alexis shared that "I had to spend two and a half grand to get rid of this awful awful stuff that was growing on my face," as she could not access public funding for this treatment. ${ }^{55}$ Some participants noted that information about ancillary treatments was often difficult to access:

Penny: I found out that you can get voice therapy through [local DHB], but that was the first time I'd ever heard of it, so it's hidden information, things that no one can find out because it's never written down anywhere, and you only know if you know people who've been exposed to it themselves, who tell you.

Here, Penny calls attention to one of the most difficult aspects of navigating genderaffirming healthcare: access to information. With the exception of the Northern Region Trans Health Services webpage, at the time of the interviews there was no DHB-specific information about what gender-affirming healthcare services were available, or how to go about accessing them. Penny proposed that a national-level service would be the best way to improve gender-affirming healthcare provision in Aotearoa, as "it means you don't get different DHBs with different levels of care."

\section{The effects of waiting}

All participants who had accessed gender-affirming healthcare described their experiences of gender dysphoria as inextricably linked to their mental health. Willow noted that "dysphoria, I believe, wrecks me," while other participants reflected that they had felt for a long time that something was not right, but had taken a while to understand this feeling as gender dysphoria. Henry described this as "feeling

\$3 million allocated over four years for gender-affirming surgeries, in response to the 50 year waitlist (Daly, 2019). As of October 2019, 198 people remained on the genital surgery waitlist (Small, 2019).

${ }^{55}$ After a thorough search, I could find no official rationale for the lack of funding for laser hair removal as a gender-affirming healthcare service. 
uncomfortable in my body" and "feeling out of place and not knowing what it was." Alexis shared:

Alexis: That was when the gender dysphoria started picking up, started to become quite intolerable, it was like having a stone in your boot and you can't get it out and there was something really wrong and I couldn't figure it out.

Almost without exception, by the time participants sought out gender-affirming healthcare, they had been struggling with gender dysphoria for several years. Participants typically described gender-affirming healthcare as the only option for reducing this distress, not only because it helped them to feel more at home in their own body, but because they were able to pass, reducing the anxiety, stress, and potential physical risk associated with social interaction, or being in public spaces. Alexis explained before she had accessed gender-affirming healthcare, "when you go around in public people stare at you like you're some kind of fucking spectacle.” Participants often noted that the concept of passing is problematic; Henry, for example, described this as "inherently transphobic," as it assumes that someone is not successful in their gender unless they fit societal norms around gender expression. Participants noted, however, that the ability to pass made them feel safer in the world, and that "it's unfair to judge trans people for trying to keep themselves safe" (Kimi).

When participants had to wait for months, or even years, to access genderaffirming healthcare, this had profound negative effects on their mental health and wellbeing. Indeed, accessing gender-affirming healthcare is associated with dramatic reductions in gender dysphoria and suicidal ideation (for a meta-analysis, see Murad et al., 2010). Penny shared that she attempted suicide during the process of accessing hormone therapy. She explained:

Penny: The reason why this got so bad and I ended up hospitalised was the waiting, because I had my birthday in between and I felt I was getting older, and HRT works better if you're younger, and just everything sort of was falling apart, and it was too much for me.

Penny's comment that "HRT works better if you're younger" refers to the fact that hormone therapy cannot reverse the prior effects of androgen on the skeleton and vocal cords (Gooren, 2011). As such, Penny was aware that beginning hormone therapy was 
time-sensitive; the longer she waited to begin hormone therapy, the more irreversible changes to her pelvic structure, height, hands, and feet. In this way, delaying genderaffirming healthcare not only has implications for mental health while patients are waiting, but also later in life, as they continue to grapple with dysphoria.

Lesley also discussed the time-sensitive nature of accessing hormone therapy, noting that "there's the irreversible bodily changes, you can't shrink bones, like I can't get surgery to make my feet fit heels, it's just a gone thing," while Kimi shared that she was facing severe mental health issues at the time she was seeking hormone therapy, "but the only thing that was really urgent to me was HRT, as I was going through male puberty and that was extremely traumatic." She explained that treating her depression, anxiety, and psychosis symptoms could wait, as "they're not really important relative to permanent changes happening in my body, that aren't being addressed."

\section{Summary}

Participants described New Zealand's gender-affirming healthcare services as inadequate to meet the needs of trans people in Aotearoa. Many who had accessed hormones reported lengthy waiting times before and between appointments, and several participants saw access to publicly funded gender-affirmation surgery as an impossibility, despite the fact that this is ostensibly funded by the Ministry of Health. Some participants described their pathway to accessing hormone therapy as unusual or unorthodox, highlighting the inconsistencies in care that can occur due to a lack of national medical pathways. Participants also noted that access to care is, in part, determined by a "postcode lottery," where trans people in large centres have easier access to care than those in smaller towns or rural areas. The barriers to accessing gender-affirming healthcare have significant implications for the health and wellbeing of trans New Zealanders, and call attention to the urgent need for increased funding for trans healthcare.

\section{Theme Two: Proving Gender}

This theme encapsulates participants' talk about the content of mental health assessments that are typically part of the process of accessing gender-affirming healthcare. As mentioned above (see pages 126-129), at the time of interviewing, best practice guidelines for providing gender-affirming healthcare rejected the pathologising 
model of care (which focuses on ascertaining whether clients are "truly trans") in favour of a model of care which focuses on the presence of gender dysphoria, and on patients being informed of the risks and benefits to treatment and capable of making their own health choices. Despite this, participants commonly described assessment experiences that aligned with the pathologising model, in which they felt they had to prove their gender in order to access care.

\section{“They're trying to make sure you're really trans"}

Participants commonly reported that their mental health professionals seemed interested in establishing whether they were "really trans" or "trans enough" during their assessment for gender-affirming care:

Henry: The psychologist should take you seriously instead of going out of their way to sort of prove you're trans (...) They're always second guessing you and trying to really really really make sure that you're really trans and really ready to commit when it's not like you haven't said that 200 times already.

Here, Henry notes that describing himself as trans was not sufficient for his health professionals to acknowledge his trans identity. Instead, his psychologist asked a series of questions Henry saw as designed to "prove" his gender identity. Several other participants described similarly lengthy conversations in which their health professionals questioned them about how they knew they were trans, or if they were sure they were trans. Theo stated, "they seemed to have endless questions about gender," and described the assessment process as "exhausting." "I hated the question 'are you sure?' because why am I here then, honestly, I wouldn't be seeking this if I wasn't sure".

Hugo stated that he was so consumed with worry leading up to his mental health assessment that he noted his thoughts down on his phone for his mental health professional to read:

Hugo: I went to him and I was like "what if I'm not trans enough? What if you're not gonna give me what I want? What if I have to go find someone else cause I've already waited for three months to see you and what if you're not gonna let me do the thing I want to do? What if I'm not man enough?" blaarrgghhh! And then I wrote on my phone cause I was too like, scared to say it, I was just 
heartbroken so I just like gave him my phone and I was like "just read this thing that I wrote to you!"

Hugo's account emphasises how nerve-wracking and painful mental health assessments for gender-affirming healthcare can be for trans people. Hugo accessed genderaffirming healthcare outside of New Zealand, but his perception of the mental health assessment as a test to be passed (rather than as an opportunity to access support and make plans for the future) closely reflected the talk of other participants. Hugo's use of "heartbroken" illustrates that gatekeeping by mental health professionals not only has the potential to deny clients access to the healthcare they need, but to exacerbate their existing distress.

Many of the assessment questions that participants described seemed to reflect attempts to establish when participants began to experience gender dysphoria, or describe themselves as trans. Henry, for example, noted that he felt an expectation on the part of his mental health providers to pinpoint the time at which he realised he was trans:

Henry: It's a really hard question to answer, how long have you known. I usually just say that I've known since I was about 16, but it's not something that you can just pinpoint (...) it's not something like, you can just put your finger on and be like - March 2007 is when I knew at 12.51 pm (laughs) it's not like that.

Henry explained that now, when he looks back on his life as a younger person, he recognises many of his feelings as internalised gender dysphoria, but at the time had not yet understood his trans identity. Here, he highlights that this does not make his gender identity invalid; that many trans people experience gender dysphoria for years before labelling it as such, so attempts on the part of mental health professionals to establish how long someone has been identifying as trans are largely irrelevant in the process of providing gender-affirming healthcare.

While some participants noted that questions around gender dysphoria were asked in a respectful and sensitive manner, others reported that their mental health professional's questioning had felt insensitive:

Alexis: I was often asked by different people the same questions, over and over and over again, and this one doctor, apparently big advocate, no tact, who 
would go "so do you want a vagina? Do you want to get rid of your penis and have a vagina instead?" and by just saying that you can get the grossness, right? So I got asked a lot of those kind of questions, and I felt like I had to answer them.

Alexis recounted the doctor's words in an indelicate, abrupt fashion, checking that I understood the unpleasant nature of the interaction ("you can get the grossness, right?") Alexis' impression of the doctor suggested that their questions were not only phrased poorly but were delivered in a tone of voice that indicated a complete lack of awareness about the sensitive nature of their questions. Alexis' experience reinforces the importance of subtle signals of safety discussed in the previous chapter (pages 84-96), including tone of voice and body language. Also of note in the above extract is Alexis' comment that she felt like she had to answer the questions. Although consumers of health services in New Zealand are not obliged to answer any question asked of them by a health professional (Health and Disability Commissioner Act, 1994), Alexis was concerned that a failure to answer questions might threaten her access to the healthcare she needed. Other participants described feeling the same way about answering questions they were uncomfortable with, highlighting the difficulties of obtaining free consent within a system where health professionals hold the power to make decisions that have significant impact on their patients' lives and wellbeing.

Although all participants had initially sought gender-affirming healthcare as adolescents or adults, several participants described questions they were asked about the clothes they wore and the toys they played with during childhood - "I was asked stuff like, so did you do a lot of dress up when you were a kid? Did you cross dress when you were a child, and did you like to play with dolls?" (Alexis). Henry also noted that he was asked several questions about his childhood, summarising this line of questioning in his assessment with the joke, "you know, did you know from age two onwards that you weren't cis?" It is possible that, with these questions, professionals were attempting to confirm that clients' gender dysphoria was "persistent" (one of the criteria for accessing hormone therapy put forward in the WPATH standards v7; Coleman et al., 2012, p. 34). In the WPATH standards, however, the only guidance as to what constitutes "persistent" gender dysphoria is a reference to the (then upcoming) DSM-V criteria for gender dysphoria, which states that "six months is the time period of full 
symptom expression required for the application of the gender dysphoria diagnosis." (p. 71). As such, it is unlikely that health professionals would be required to establish the existence of gender dysphoria in early childhood in order to recommend access to care for adolescents or adults.

As well as a focus on childhood experiences, participants reported a focus on their gender expression during assessments for gender-affirming healthcare:

Felix: In part of my psych assessment to begin testosterone my psychologist had to specify how I dressed (...) and when I started dressing as the other gender.

Henry: They mentioned how I dress, what colour my hair was, the fact I have piercings, my body language, how I sit, how I talk, how I speak (...) It's like, why do you need to know what colour my hair is, or the fact my nose has two piercings, like wow, if I only had one ring in my nose, would I still be trans, you know?

Felix and Henry both noted that mental health professionals seemed to view their gender expression as evidence of their gender identity. Felix and Henry went on to reflect that if gender is socially constructed (as they both understood it) then the only way to understand another person's gender is to ask how they describe themselves; it is impossible to assume gender based on someone's outward appearance. For many participants, this tendency on the part of professionals to connect gender identity with gender expression informed the way they expressed and described their gender within assessments (I discuss this further in the next section, see pages 145-148).

\section{Why is there a need to prove gender?}

The majority of participants reported that, although their assessments for genderaffirming healthcare were typically very lengthy and/or had involved persistent and uncomfortable questions about their gender, they believed that their mental health professionals wanted them to have access to the care they needed. Some participants posited that the questions asked during their mental health assessments were determined by the requirements of the "system". Henry, for example, noted that his psychologist had apologised for the invasiveness of the questions she asked; he stated, "the professionals don't necessarily agree with the questions they're asking but they know they have to ask them, cause that's how the system is." Other participants made similar 
comments, with Martin noting that the purpose of a mental health assessment was "to tick their boxes" and Hugo mentioning it was "needed for the paperwork".

As well as speculating that institutional or "system" requirements account for the nature of mental health assessments for gender-affirming healthcare, some participants posited that mental health professionals attempt to ascertain that clients are "really trans" (that is, they will always understand their gender in this way and continue to access to gender-affirming healthcare) out of concern that their client would later realise they were not trans and would subsequently regret accessing gender-affirming care (this is often referred to as 'desistance' or 'detransition'; ${ }^{96}$ Serano, 2016):

Lily: The worry is that we need to know for sure because you might mess someone's life up if you do it wrong.

Henry: It's because they're afraid of people making irreversible changes and not fully understanding the effects that, like, for example testosterone will have on the body and the fact that, you know, even if you stop taking it, you know, not all the effects are going to be reversible and there's kind of no going back.

Lily and Henry anticipate a fear on the part of health professionals that, if someone were to access gender-affirming care and subsequently experience a change in gender identity, this would have a significant negative impact on their life due to the irreversible bodily changes that can come with gender-affirming care. As Henry points out, some effects of testosterone (including clitoral growth, facial hair growth, voice changes and male-pattern baldness) are not reversible after hormone therapy is stopped (although the degree to which changes are reversible depends on how long someone has been on hormone therapy; Deutsch, 2019). Gender-affirming surgeries are also typically irreversible (Bizic et al., 2018).

Some participants challenged the idea that accessing gender-affirming healthcare and later ceasing treatment is always harmful or distressing:

Willow: It's more of a fear among professionals than among queer people, I think, it's something that they like to push on you, like the boogie man, like what

\footnotetext{
${ }^{56}$ I use 'detransition' from here onwards to describe the process of trans people later coming to identify as cis.
} 
if you're not what you think? And honestly, we're all bundles of muscle and fat walking around in meat suits, so it doesn't really matter in the large scale of things, if it's something that you need to explore, then you need to explore it, like, maybe you do detransition, maybe you do end up with slightly weirdly shaped body (...) but yeah that shouldn't be held over your head as like a threat. Here, Willow suggests that the negative impacts of detransitioning may be exaggerated by health professionals and that for some people, accessing gender-affirming healthcare might be necessary step in order to understand their gender. Nala expressed a similar sentiment, noting during a discussion of detransitioning that "maybe even they needed to like, go through that process".

In conversations around 'treatment regret' many participants drew on the idea of gender as a journey (Temple Newhook et al., 2018). They reflected that to move through different ways of identifying or understanding one's gender does not reflect a failure or mistake, either on the part of health professionals who provided genderaffirming care, or the person who has received the care:

Star: There's just a lot of fear that if somebody transitions and then changes their mind what if the doctors are held liable for not like, ruling out all factors? (...) I don't really resent them or anything, cause first of all I don't regret any of the stuff that happened, cause it was what worked for me then, and I'm not having any problems relating to it now, honestly, I'm fine with it. (...). I don't regret getting top surgery and I have people ask me sometimes like "but now that you're kind of being a woman again, like isn't it horrible not having this huge womanly part of you?" First of all, that's not, that's not really a womanly part of me, it's just a part of me, and second of all I can wear so many more shirts now! (laughs).

Star, who previously identified as a trans man (accessing gender-affirming healthcare overseas), shared that she believed her health professionals had acted in accordance with best practice, as they had given her the care she needed when she needed it. Of course, Star's experience may represent the best possible outcome for someone who accesses gender-affirming healthcare, then later decides to stop doing so. Others in a similar situation have spoken out about their experience, sharing that they felt their health professionals should have asked more questions about their gender and 
experience, slowed the process down, or refused to allow them access to genderaffirming healthcare altogether (see, for example, Callahan, 2019). ${ }^{57}$

The wider conversation about treatment regret and detransitioning highlights that there will likely always be a group who feel that health professionals have not practiced as they should have; on one hand, gatekeeping delays (and in some cases, prohibits) access to gender-affirming care, which is incredibly distressing for those who need it, while on the other hand self-identified detransitioners argue that making care more accessible increases the likelihood that someone might access care and later feel this was the wrong decision (Singal, 2018). Alexis noted that health professionals tend to lean toward the former, stating that "there is this attitude that it is better to deny thousands of trans people, than to let one cis person transition and regret it."

Although most participants believed their mental health professionals wanted them to access the gender-affirming healthcare they needed, one participant reported that her mental health professionals actively discouraged her from accessing genderaffirming care:

Kimi: They were avid gatekeepers, their only role was to stop me by any means from getting hormone replacement therapy. They would make up lots of excuses, because I was 17 at the time they were saying that somebody under 18 can't really think for themselves (laughs), they were saying that I should wait until I've finished my male puberty in case I change my mind, and that went on for two and a half years, and their sole purpose was just to destroy lives out of an ideological drive, and I honestly think they should lose their medical license.

\footnotetext{
${ }^{57}$ Empirical evidence suggests that number of people who experience regret or detransition after accessing gender-affirming healthcare is very small. The Amsterdam Cohort of Gender Dysphoria Study reported that, among the 6,793 people who attended their gender identity clinic between 1972 and 2015, "only $0.6 \%$ of transwomen and $0.3 \%$ of transmen who underwent gonadectomy were identified as experiencing regret" (Wiepjes et al., 2018, p. 1). Although $8 \%$ of the 27,715 participants in the 2015 US Trans Survey reported de-transitioning at some point in their life, only $0.4 \%$ of the overall sample had done so because they realised gender transition was not for them - the remainder had detransitioned due to pressure from a parent, partner, or other family member, because they struggled to gain employment, or because they faced discrimination after they began transitioning (James et al., 2016). I do not cite these small numbers to suggest the experiences of detransitioners are unimportant, but to highlight that because regret and detransition is so rare, it is arguably near-impossible for health professionals to predict who will later decide to stop their gender-affirming healthcare.
} 
Importantly, Kimi did not believe that her mental health professionals were attempting to delay her access to hormone therapy, but to prevent it altogether. She reported that when she turned 18, her mental health professional refused to refer her to an adult mental health service, keeping her on at the youth mental health service instead. Kimi eventually began hormone therapy after moving to a larger city, where she enrolled at a local sexual health clinic.

Throughout the interviews, and later during transcription and analysis, I was troubled by extracts like the one above. On one hand, it seemed impossible to me that a mental health professional would be driven by a motivation to destroy their clients' lives. On the other hand, the view that gender-affirming healthcare is an inappropriate response to gender dysphoria is not uncommon, at least at the societal level, so it seemed plausible that some mental health professionals might hold this view. Kimi's experience was further complicated by the fact that she had presented to youth mental health services for a hormone therapy referral at a difficult time in her life; she reported that she was homeless, had a history of abuse, and was experiencing symptoms of depression, anxiety, and psychosis. I wondered if she had been denied care because her mental health professionals had felt that she was not in a safe or stable place to access hormone therapy; however, she did not say that they had presented this as a reason. Perhaps the most important learning to take from Kimi's experience is that she did not feel that her gender was validated by health professionals at her youth mental health service, and that regardless of the reasons she was refused access to services, validation of gender is central to trans-affirmative care.

\section{Summary}

When discussing mental health assessments for gender-affirming healthcare, participants commonly described feeling a need to prove their gender to mental health professionals in order to receive access to the care they needed. Participants reflected they felt this imperative because of repetitive questioning about their certainty about their gender, when they first understood themselves as trans, how they experienced their gender in their early life, and how they expressed their gender at the time of the assessment. When asked why they believed professionals had asked these repetitive questions over the course of assessment, some participants shared that the system required their health professionals to do so. Others noted that health professionals are 
afraid their clients will experience 'treatment regret', so ask questions designed to ascertain whether clients will later change their mind about their need for genderaffirming care. One participant felt her health professionals had restricted her access to care because they did not agree with gender-affirming healthcare provision, highlighting the need to affirm clients' gender and healthcare needs, regardless of whether that care is provided at the time.

\section{Theme Three: The Trans Narrative}

Almost all participants who had accessed gender-affirming healthcare shared that, because assessment questions seemed focussed on establishing that they were "truly trans" or "trans enough", they felt there was a particular story they had to tell about their gender in order to access care:

Henry: I guess what [the questions do] is put a lot of pressure on trans people for feeling like they have to fit a sort of perfect mould, and that's why I ended up, not really lying on impulse, because later when I thought about it I was like hey no I was right, but just getting scared that if I didn't appear completely mentally stable or completely $3000 \%$ sure of my gender, that they would just not take me seriously because of the sort of style and approach of the questions.

Henry's use of the word "scared" emphasises the high stakes involved in mental health assessments for gender-affirming care. Henry describes fears that his professional would believe he was unsure of his gender, or not well enough to make decisions regarding his gender, and deny him hormone therapy as a result.

Several other participants also shared that they felt pressured to fit this mould or conform to a typical narrative in order to access the care they needed. Although participants' perceptions of the trans narrative varied slightly, all agreed that this involved having a binary gender identity, knowing they were trans since a very young age, and wanting a 'full' medical transition (that is, hormones, genital surgery, and top surgery if applicable):

Felix: If you don't want to have the full medical transition, then you're automatically disregarded, you're not valid, it was brought up for me a lot as 
well, I was constantly being asked, "how far do you want to go with the surgery?"

Felix notes that, for many health professionals, wanting genital surgery is a necessary part of identifying as trans. This reflects a pathologising perspective (and the medicalisation of trans bodies) described previously (see pages 17-20 and 127), where the purpose of gender-affirming healthcare is to 'fix' the 'problem' of trans people being "born in the wrong body" (Johnson, 2015, p. 807). Contrary to queer-theoretical understandings of gender, which do not see gender as necessarily connected to aspects of one's of physical body, this perspective considers aspects of one's physical body (e.g. breasts, genitals, and facial hair) as inherently gendered. The pathologising perspective does not allow for trans people to be content with any part(s) of their body that do not 'match' their gender identity and invalidates the gender of trans people who are content with any 'gendered' aspects of their body.

Several participants explained that they either had put forward the trans narrative in order to access care, or would in future, even if that narrative did not fit with their story. Bats, who identifies as nonbinary, for example, shared that:

Bats: I want top surgery, but on my letter to apply for top surgery I'm going to be a man. I'm a man, I want my boobs gone because boobs aren't for men, and I'm gonna be the manliest man, and I'm just gonna say that, so I can get my surgery.

Here, Bats reflects that New Zealand's healthcare system typically draws on a binary understanding of gender to inform decisions around gender-affirming surgeries. For Bats, presenting their nonbinary identity to healthcare professionals may be a risk; instead, it is preferable to claim a male gender identity and have a higher chance of accessing the care they need. Several other participants shared experiences along the same lines and noted that embodying the dominant trans narrative was not only about how they described their gender identity, or gender journey, but about how they expressed their gender in session.

Willow: You've got to present the facade of the 50s housewife if you want to get anywhere. With all the uncomfortableness of that, the connotations of that (...) you have to basically play on a bunch of stereotypes. One of my friends was 
being the older cynical trans, and she was like yeah just, if you're going to go to another one of those appointments, the first thing you do is go full high femme, you know, long skirts and high heels and way too much lipstick, et cetera.

Willow's experience of hearing advice from older trans community members was common among participants. Many reported that they had been told by those who had sought gender-affirming care in the past that it was important to dress in a hyperfeminine or hypermasculine manner to their appointments, even if that was not how they usually expressed their gender, in order to avoid any doubt on the part of professionals that they were 'truly' trans.

Participants' reports of re-telling the trans narrative highlights a potential difficulty in making progress towards trans-affirmative healthcare; there are health professionals who no longer subscribe to the trans narrative and understand that gender expression does not determine gender identity. ${ }^{58}$ If trans people continue to present themselves according to a narrative at the advice of others who have accessed care, however, there will be little opportunity for trans people to present their gender authentically and learn that they will still receive the care they need - information they can, in turn, feed back to their communities. Another potential consequence of presenting this narrative is that the narrative is reified for health professionals; if health professionals consistently hear similar stories from their clients, this might increase the likelihood they will expect the same story from future clients.

Participants shared that the pressure to conform to a dominant narrative felt unjust, as access to gender-affirming healthcare should not be restricted to one type of trans person. They argued that, because the trans community is so diverse, care should be available to all who need it, regardless of how they experience or express their gender. Lily shared that pressure to tell the trans narrative not only made her feel uncomfortable, as it was inconsistent with her experience of gender, but also limited what she was able to discuss with health professionals in later sessions:

Lily: I've had this experience where you kind of set yourself up as the relatively typical trans person, we tell the typical narrative of playing with girls' toys when

\footnotetext{
${ }^{58}$ I know this because I am one myself and have met many others!
} 
you're little and trying on mum's makeup and all that kind of stuff (...) but having said all of that, it means that I now can't come back later and say "I want to retain the use of my penis" because it's like oh but you're the typical trans woman who's always felt wrong in your body and wants a different body and all the stuff, and it's like, well actually no I'm quite okay with my body.

Lily went on to explain that she was dissatisfied with the effect that hormones had on her sex drive but felt unable to discuss a change in prescription with her doctor, as "trans women are supposed to not like their genitals" so should not want to use them for sex. Lily noted that trans women are "supposed to kind of have one or the other" - that is, trans women are expected to sacrifice either their sex life or their hormone therapy. As a trans woman who enjoys sex using her penis, Lily not only challenges discourses about what is means to be a woman (as women are commonly perceived as having less sexual desire than men; Allen, 2003), but also resists transnormativity by refusing to renounce her genitals "as sites of sexual pleasure" (Vipond, 2015, p. 26). As Vipond (2015) points out, however, this resistance comes at the potential cost of losing her access to gender-affirming healthcare.

\section{Summary}

As a result of the focus on 'proving' gender outlined in theme two, participants shared that they often felt pressure to conform to a dominant trans narrative during mental health assessments for gender-affirming healthcare (having a binary gender identity, knowing they were trans since a very young age, and wanting a 'full' medical transition), even if it did not reflect their own experience. Adherence to this narrative restricted the extent to which some participants could later seek support from health professionals. Following such a narrative may also create a cycle in which trans people carefully monitor their gender expression in healthcare contexts where this is no longer necessary, as they have heard from other trans people that expressing gender in an authentic manner comes with a risk of being denied access to care.

\section{General Discussion}

Participants' talk about their experiences of accessing gender-affirming healthcare described a system in which publicly funded care is dependent on geographical location and, even when this is available, waiting times often stretch to 
months or years. The majority of participants saw a mental health professional for an assessment in order to access gender-affirming healthcare, and typically understood these assessments as designed to prove they were "really trans" or "trans enough". Participants tended to attribute this need to prove gender to the requirements of the system, or to a fear on the part of their health professionals that they would later regret their decision to access gender-affirming care. As a result of professionals' focus on participants' gender identity and expression, many reported that they felt pressure to present their gender in a way that fits a dominant trans narrative, where they had known they were trans from a young age, expressed their gender as hyperfeminine or hypermasculine, and wanted all available medical transition options.

\section{Participants' Experiences in Context}

The experiences of participants in the current study were remarkably consistent with the gender-affirming healthcare experiences of trans people reported in international literature. Structural barriers to accessing care have been documented in the US (Safer et al., 2016), Canada (Giblon \& Bauer, 2017), and the UK (Ellis et al., 2015), with research participants commonly sharing that they, like participants in the current study, were often told care was not available to them, or was available but came with lengthy waiting times. The experience of being asked repetitive questions about gender and feeling a pressure to present gender in a way that conforms to the dominant trans narrative has also been widely reported in international literature on accessing gender-affirming healthcare (Ellis et al., 2015; McNeil et al., 2012; Violeta \& Langer, 2017).

There is a notable lack of New Zealand-based research that explores the experiences of trans people who have accessed gender-affirming healthcare, however the structural barriers to care faced by participants in the current study mirror findings from the Counting Ourselves survey (Veale et al., 2019), and reflect the stories told in popular media (e.g. Holden, 2016), in the Human Rights Commission Report of the Inquiry into Discrimination Experienced by Transgender People (2008), and trans advocates' accounts of common experiences (Wi-Hongi et al., 2017).

It must be noted that gender-affirming healthcare services are not the only services that have been described as a 'postcode lottery' within New Zealand's public 
health system; this term has also been used in reference to aged care (New Zealand Labour Party, Green Party of Aotearoa New Zealand, \& Grey Power, 2017), cancer treatment (Duff, 2017), and hip and knee replacements (Goodwin, 2016).

Inconsistencies in care may be an inevitable consequence of the structure of New Zealand's elective services system. For all medical and surgical services that do not require urgent attention, patients are given a priority score out of 100 based on their level of need for and ability to benefit from care compared to other people (Ministry of Health, 2016). This score determines whether patients' treatment is funded. Each DHB sets its own threshold for funded treatment (e.g. a priority score of 40 or above results in funded treatment for a specialist service), meaning that the same priority score could result in a patient being denied treatment in one DHB, and approved treatment in another. It is likely, however, that gender- affirming services are disproportionately affected by the 'postcode lottery' issue, as it is unclear how practitioners should use current prioritisation tools to make decisions about the level of need for and ability to benefit from gender-affirming healthcare.

\section{Implications, Reflections, and Future Directions}

My analysis not only provides insight into the experiences of trans people in Aotearoa, but also the difficulties of accessing gender-affirming healthcare in the New Zealand context, and the complexity of attempting to understand this process within an ever-changing system characterized by underfunding, lack of information, and confusion regarding availability of care. In the current study, participants' experiences of accessing gender-affirming healthcare deviated significantly from what would be expected according to best practice guidelines. This may be (at least in part) attributable to the fact that when these interviews took place, there were no national guidelines for gender-affirming healthcare provision. The Ministry of Health made information for health professionals available on their website to assist them in this process (Ministry of Health, 2018d), however this information was consistent with the WPATH v6 Standards rather than the updated v7 Standards, meaning it was not consistent with international best practice. ${ }^{59}$ It may be that this oudated information provided by the Minstry of

\footnotetext{
${ }^{59}$ For example, the website noted that real life experience ("living and working full time for at least two years as a woman/man”) was required to access hormones and surgery. Wi-Hongi and colleagues (2017)
} 
Health can account for the experiences of participants that often seemed to be consistent with a pathologising model of care, rather than a diagnostic model of care. Without speaking to mental health professionals involved in the provision of gender-affirming care, however, it is impossible to know what model (if any) they were following, where they accessed information about healthcare provision, and the purpose of their assessment questions. Future research should explore mental health professionals' perspectives about their role in providing gender-affirming healthcare in New Zealand.

This analysis supports the need for increased resourcing for gender-affirming healthcare provision, as well as for establishing clear treatment and referral pathways. This study also highlights the importance of taking a non-pathologising approach to gender-affirming healthcare provision and ensuring that all health professionals in New Zealand share a common understanding of the purpose of mental health assessments for gender-affirming care, as well as what these assessments involve. There has been some progress in this regard since these interviews were conducted, with the publication of Oliphant and colleagues' (2018) guidelines for gender affirming healthcare for gender diverse and trans children, young people and adults in Aotearoa. The guidelines are not intended to replace WPATH guidelines, but to "present additional guidance for the provision of gender affirming healthcare in Aotearoa, New Zealand" (p. 6). There is still ambiguity in these guidelines, however, as they present the WPATH criteria for accessing care (which include experiencing gender dysphoria), and also refer to the informed consent model (which does not require experiencing gender dysphoria). As such, there is still a need for New Zealand-based guidelines that provide specific advice to mental health professionals conducting assessments for gender-affirming care, to advise them of their role, and what should be included in their assessment.

There is ongoing debate as to whether health professionals providing genderaffirming healthcare should adopt an informed consent approach (which could mean that mental health assessments cease to exist altogether), rather than continuing to use

point out that "attempting to be read as the correct gender without appropriate medical support can have negative consequences" (p.156). These include exposure to physical and sexual violence, high social anxiety due to hypervigilance, and physical problems such as back pain and compressed ribs from binding one's chest too tightly, or bladder infections as a result of avoiding public bathrooms. Due to these potential negative consequences, real life experience was removed from the WPATH Standards of Care in 2011. 
the diagnostic model of care. Advocates of informed consent argue that there is a need to move beyond diagnosis in order to "counter the negativity attached to trans embodiment in medical discourse" (Davy, 2015, p. 1166). According to this view the only way to entirely de-pathologise gender-affirming healthcare and respect the selfdetermination of trans people is to view medical transition as a human right (Stop Trans Pathologization, 2012).

Many oppose this move to informed consent on the basis that moving entirely away from diagnoses will threaten access to care, given that medical insurance companies generally refuse to fund services in the absence of a diagnosis (Vance Jr et al., 2010). The move toward informed consent is somewhat less controversial in New Zealand, presumably because our public health system has the power to approve funding for gender-affirming care in the absence of a diagnosis. However, I think it is important to note that gender-affirming care is already widely portrayed in mainstream media as 'elective' (Webb-Liddall, 2019). As such, removing the 'distress' criterion from this process may make public funding for gender-affirming care far more difficult to justify. On top of this, health professionals may be wary of using the informed consent model in their practice given that international standards of care (Coleman et al., 2012) do not currently endorse this model, and also because there is a lack of longitudinal research about the outcomes of clients who access care through informed consent (Schulz, 2018). It may be that health professionals in New Zealand need to use a trans-affirmative diagnostic model for the time being, but will be able to move towards the informed consent model in the future with the support of revised WPATH guidelines, more research about the efficacy of informed consent, and increased societal awareness of the importance of gender-affirming healthcare.

\section{Conclusion}

I have provided an in-depth understanding of the experiences of 13 trans young adults living in New Zealand who have accessed gender-affirming healthcare. Participants described significant structural barriers to accessing care. They also noted that they often felt a need to prove their gender, and to present their gender according to a dominant narrative, to access the care they needed. These experiences closely reflect those reported in wider literature, and call attention to importance of increased funding 
for gender-affirming healthcare, and clear referral and treatment pathways to avoid a "postcode lottery" of care. The current study also raises questions about the role of mental health professionals in providing gender-affirming healthcare, which continues to be hotly debated by researchers, mental health professionals, and trans advocates.

In the next chapter I present the results of a (predominantly quantitative) online survey about rainbow peoples' mental health support experiences. In this survey I also explore participants' experiences of accessing gender-affirming healthcare and consider whether the experiences of the 13 participants discussed in the current chapter are reflected by a wider group of trans New Zealanders. 


\section{Chapter 8: Online Survey}

Based on analysis of the interview data, I developed and conducted an online survey about rainbow experiences of accessing mental health support, and trans experiences of accessing gender-affirming healthcare, in Aotearoa. The purpose of the survey was to develop an understanding of the needs and experiences of a wider group of rainbow community members, to ensure the resource I subsequently developed was based on the stories of as many rainbow people as possible. In this chapter I document the process of feeding analysis of interview data back to rainbow advisors, designing the survey, and distributing the survey. I then present an overview of key survey findings and discuss how these compare with my interview analyses and the wider research literature.

\section{Survey Development}

\section{Feeding Back Analysis of Interview Data}

After I had identified nine preliminary themes from interview data (see Chapters 6 and 7) I held a series of hui with community organisations and community leaders to (1) share the analyses, (2) check whether the analyses fit with their personal and professional experience, and (3) ask whether they felt I was missing anything they expected to see in the analyses. Without exception, community advisors were unsurprised by the findings I presented, and noted that they fit closely with their experience working within rainbow communities. They were pleased to have New Zealand-based data that reflected what they already knew and commented that they could refer to these findings in their work. During these hui we also discussed the voices that might be absent from my analysis, including rainbow people who lived rurally or had disabilities, as well as minority ethnic groups (with the exception of Māori). Community advisors emphasised the importance of trying to reach these groups as we recruited participants for our online survey, in the hopes of filling some of the gaps in my data collection so far. 


\section{Survey Design}

Following feedback of the interview study, I began to develop an online survey based on analysis of the interview data. First, I used my thematic map to list groups of potential measures. For example, from the theme Therapy as a Microcosm I noted the need for measures assessing participants' comfort sharing their gender, sex characteristics, and sexual orientation with mental health professionals, and comfort discussing issues related to gender, sexuality, and sex characteristic diversity. From the theme Signalling Safety I created a list of potential actions from mental health professionals (e.g. asking about gender in an assessment) in order to ask how helpful each one would be when meeting a new mental health professional.

To inform the structure and wording of the survey I examined similar studies by McCann and Sharek (2014), Liddle (2000), Shipherd, Green, and Abramovitz (2010), and Counting Ourselves (the Aotearoa New Zealand Trans and Nonbinary Health Survey, which was running at the time I developed my online survey; Veale et al., 2019). Some survey items were adapted from items in these past studies, however the majority of survey items were developed based on the experiences shared by participants in the earlier interview study. I took the survey draft to RainbowYOUTH, InsideOUT, and Gender Minorities Aotearoa, as well as to a number of other community advisors, and made changes in response to their feedback. ${ }^{60}$

\section{Survey Recruitment}

This project was approved by the Victoria University of Wellington Human Ethics Committee (RM\# 0000024394; see Appendix F). For the online survey, I felt it was important for us to start recruitment at age 14 (rather than at 16, like the interview study) for two main reasons. Firstly, rainbow community organisations had shared that they are routinely contacted by young people under the age of 16 for support,

\footnotetext{
${ }^{60}$ In response to feedback I reworded several questions and added items about participants' experiences of accessing support from rainbow organisations, whether intersex participants had accessed medical care related to being intersex (and their experiences of this), and methods of currently accessing support (e.g. iwi-based services). I extended the list of intersecting identities to include sex workers and faith or religious identities. I also added a series of items to the section on gender-affirming healthcare about Western/Pākehā understandings of gender within mental health contexts and participants' experiences of pressure to conform to the trans narrative.
} 
particularly around coming out and navigating relationships with friends, partners, and whānau. I thought it essential that we understand the needs and experiences of younger rainbow community members, to provide them with the best possible mental health support during early adolescence. Secondly, in our interview study participants shared that they had sought support at the age of 15 or under and had found their mental health professionals invalidating or rejecting. By asking about these experiences in our online survey, we could use our data to push for change. Moreover, I felt that sharing these experiences would make the young people feel respected and listened to. The Human Ethics Committee expressed significant concern about allowing 14 and 15-year-olds to take part in our survey without parental consent but granted approval for this following revisions to the survey and ethics application (see Appendix $G$ for a detailed description of this process).

Before beginning recruitment, I invited participants from my interview study to participate in a pilot survey. Interview participants were offered a koha of a \$20 Prezzy card to thank them for taking part. I conducted the pilot survey to determine whether any final changes should be made to improve survey content or usability, as well as to maintain connections with the people that had shared their stories with me the previous year. The pilot survey began on October 1st, 2018, and pilot participants were asked to complete the survey within one week. 27 of the 34 people invited to be part of the pilot survey did so. Based on feedback from the pilot survey we fixed a few typographical errors and formatting issues and clarified wording at points in the survey.

The survey was launched on October $9^{\text {th }}, 2018$ and was available online until November $12^{\text {th }}$. Potential participants were recruited using a flyer (see Figure 6) similar to that used for our interview study. RainbowYOUTH, InsideOUT, and Gender Minorities Aotearoa posted this flyer to their social media sites, and I sent the flyer to other rainbow organisations, mental health organisations, community organisations, and contacts I had made over the course of conducting my research. I also emailed the flyer to libraries and schools around Aotearoa, and professional organisations such as the New Zealand Association of Counsellors, New Zealand College of Clinical Psychologists, and New Zealand Psychological Society. Physical flyers were posted around Victoria University of Wellington, the University of Auckland, and Auckland 
University of Technology, as well as in cafes and on community notice boards in Auckland and Wellington.

At the end of the survey, participants were invited to click through to a second short survey if they wanted to enter a prize draw for a $\$ 50$ voucher, receive a copy of the findings and updates about the project, help to develop the later resource, receive invitations to participate in more research, or share the survey with others. With ethical approval, on the Facebook page I created for this project (and on my own social media accounts) I employed creative strategies to encourage sharing of our research flyer by posting photos of various animals (mine and my friends and colleagues' pets) with our research flyer. I would then regularly post these images with captions (see Figure 7 for an example). These posts ensured that survey recruitment remained steady across the time the survey was running. I also paid to boost survey recruitment posts on Facebook twice during the recruitment period (for a total of $\$ 40$ ).

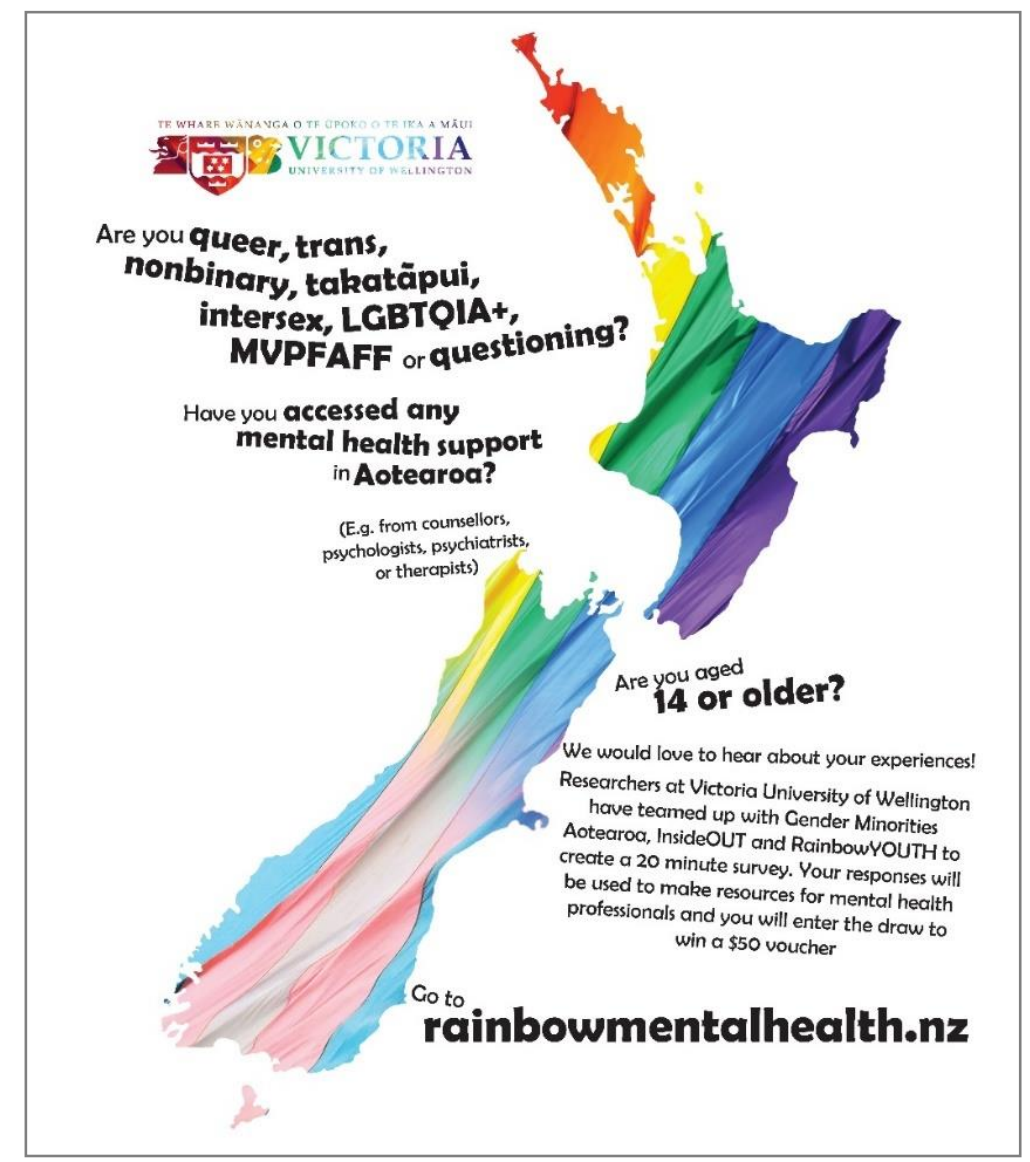

Figure 6. Online survey recruitment flyer 
Behind every research team are their fluffy supporters!

Today Poppy wants you to share our flyer - go to

tinyurl.com/shareourpost - and take part in the survey if you can at rainbowmentalhealth.nz. Help us improve mental health services for Aotearoa's rainbow communities

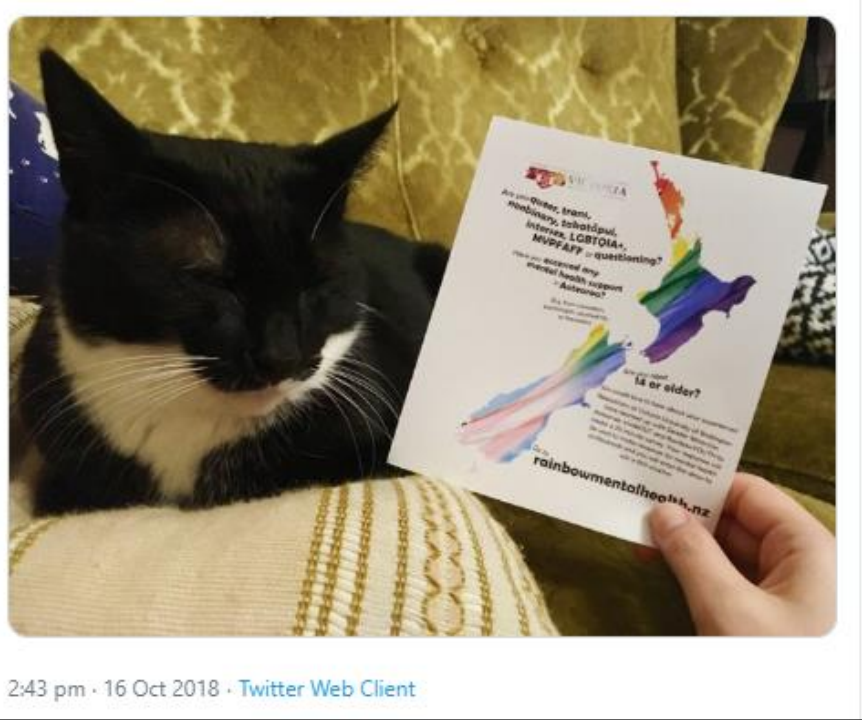

Figure 7. Tweet of Poppy (my beautiful tuxedo cat) promoting the online survey.

\section{Data Cleaning}

In total, there were 1,701 responses to the survey. Ninety-two were removed as they did not respond beyond the survey's initial demographic questions, and a further 24 were removed because they responded in a manner indicating they were not part of the rainbow community. ${ }^{61}$ I then used Veale, Peter, Travers, and Saewyc's (2017) method of removing duplicate responses, by identifying responses that shared an IP address and answered demographic questions in the same way $(n=7)$. These were likely participants that had filled out part of the survey, then later restarted the survey on

\footnotetext{
${ }^{61}$ To be excluded on this basis, participants described their sexuality as straight, heterosexual, or normal, reported they were not trans or did not understand the question, reported they were not intersex or unsure if they were intersex, and noted they had not accessed gender-affirming healthcare. I also checked their open boxes for anything to suggest they should not be excluded. It is possible that these respondents took part as considered themselves part of the rainbow community as allies or were not aware the survey was restricted to rainbow community members.
} 
a different device. In these cases, I either deleted the less complete survey, or in cases where participants had filled out the survey twice, retained the most recent response. Another response was identified as a duplicate response and removed - a pilot participant who had taken part again when the survey was made live. Finally, the shortest response times were checked for careless responding, and all responses checked for mischievous responses. Two responses were removed on this basis; one that entered gibberish such as "adf" and "asdf" in several open boxes, and another that identified their gender as "basketball" and reported that they had accessed mental health support due to "not being able to bounce". This left a final sample of 1575 .

\section{Survey Measures and Descriptive Statistics}

To spare the reader a frighteningly lengthy thesis chapter, I describe relevant survey measures and descriptive statistics below, and provide the full survey in Appendix $\mathrm{H}$ and detailed demographic information in Appendix I. Here I provide a brief overview of the descriptive data, focussing on aspects most relevant to my research question and later resource development. Unless stated otherwise, the most common form of question invited participants indicate their agreement with statements on a 5point Likert scale $(1=$ Strongly Disagree to $5=$ Strongly Agree $)$.

\section{Demographic Information and Wellbeing}

\section{Age, sexual orientation, gender, and identity centrality}

Participants were aged between 14 and $73(M=24.99, S D=9.92)$. Participants provided a total of 323 unique responses to the open-ended item assessing sexual orientation, and 285 unique responses to the open-ended item assessing gender. Sexual orientation and gender data were first coded using dummy coding (in order to alter raw responses as little as possible), then were coded a second time into discrete categories for ease of analysis (see Appendix I for details). Figures 8 and 9 provide an overview of participants' sexual orientation and gender. In response to a question about identity centrality, participants tended to agree that being queer, trans, and/or intersex is an important aspect of their life $(M=4.16, S D=1.02)$. 


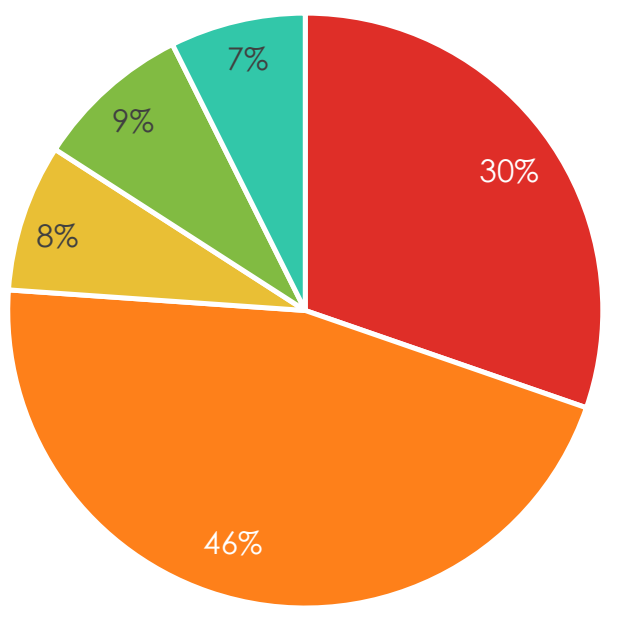

- Gay/lesbian

- Multiple gender attracted

- Asexual

- Queer

- Something else

Figure 8. Participants' sexual orientation.

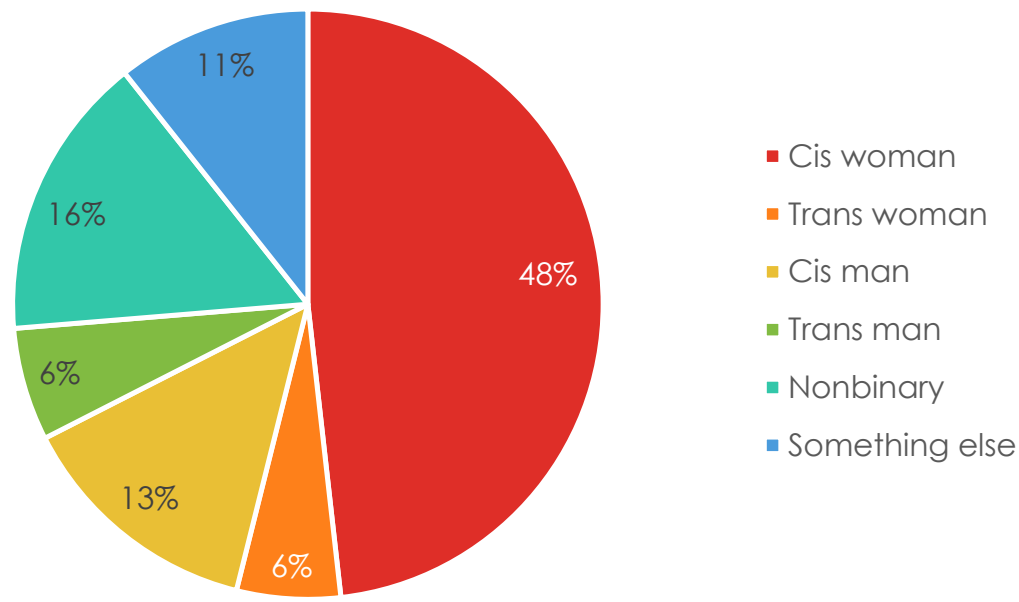

Figure 9. Participants' gender.

\section{Trans and intersex identification}

Nearly a third of participants $(n=445,28.3 \%)$ described themselves as trans or nonbinary, $976(62 \%)$ as not trans or nonbinary, and $141(9 \%)$ as unsure if they were trans or nonbinary. The majority of participants $(n=1383,88 \%)$ reported that they were not intersex or did not have a variation of sex characteristics. Twenty-five participants 
$(1.6 \%)$ indicated that they were intersex or had a variation in sex characteristics, while 115 participants $(7.3 \%)$ were unsure if they were intersex.

\section{Gender-affirming healthcare}

In total, 213 participants (13.5\%) had accessed gender-affirming healthcare. A further 43 participants $(2.7 \%)$ were in the process of accessing gender-affirming healthcare at the time of surveying, and $153(9.7 \%)$ had not accessed gender-affirming healthcare but wished to do so in future. ${ }^{62}$

\section{Ethnicity and iwi}

Participants identified with 58 different ethnicities. Of these, the most common ethnic identifications were Pākehā (86.7\%) and Māori (14.3\%). Māori participants represented 48 different iwi across Aotearoa. For the purposes of analysis, participants were coded into the following categories: Pākehā (65.9\%), Pākehā and Māori (11.3\%), Māori (3.1\%), ${ }^{63}$ Pākehā and other ethnicity (9.7\%), and Other ethnicity (10.0\%).

\section{Intersecting identities}

Participants' intersecting identities are shown in Table 1. Other important identities included having mental health difficulties, being a survivor of trauma or abuse, having a chronic illness, having or caring for children, and being unemployed. Nineteen participants specified their religious affiliation (or lack thereof). Specific mental health difficulties noted by participants included depression, anxiety, PTSD, bipolar disorder, substance abuse, and eating disorders.

\section{Region}

Participants represented every region in New Zealand, with the majority of participants living in Auckland (29.3\%), Wellington (28.9\%), or Canterbury (11.1\%).

\footnotetext{
${ }^{62}$ A table showing crosstabs between trans identification and gender-affirming healthcare access is shown in Appendix $\mathbf{J}$.

${ }^{63}$ This group included sole-identified Māori $(n=37)$ and 12 Māori participants who identified with other non-Pākehā ethnicities.
} 
Table 1

Participants' intersecting identities

\begin{tabular}{lclc}
\hline & $n(\%)$ & & $n(\%)$ \\
\hline Neurodiverse & $372(23.6)$ & Polyamorous & $329(20.9)$ \\
Low socioeconomic status & $226(14.3)$ & Faith and/or religious & $167(10.6)$ \\
Physical disability & $133(8.4)$ & Migrant & $78(5)$ \\
Sex worker & $52(3.3)$ & Homeless & $34(2.2)$ \\
Refugee migrant & $2(0.1)$ & Other important identity & $156(9.9)$ \\
\hline
\end{tabular}

Note. Polyamorous, sex worker, and homeless categories include participants who identified with these descriptions now and in the past.

\section{Subjective wellbeing}

Subjective wellbeing was measured using four items based on Durie's (1994) Te Whare Tapa Wha model. 'Te Whare Tapa Wha' translates to 'the four-walled house' and is a Māori model for understanding health holistically. Participants were asked to rate their current health/wellbeing in the areas of physical health, mental health, social wellbeing, and spiritual wellbeing. Responses were measured on a 5-point Likert scale $(1=$ Very Poor to $5=$ Very Good). On average, participants rated their subjective wellbeing highest in physical wellbeing (taha tinana; $M=3.37, S D=2.76$ ), followed by social wellbeing (taha whānau; $M=3.17, S D=4.59$ ), spiritual wellbeing (taha wairua; $M=2.93, S D=6.28$ ), and mental wellbeing (taha hinengaro; $M=2.39, S D=5.22$ ).

\section{Experiences of Accessing Mental Health Support}

\section{Accessing support}

At the time of surveying, 35\% and $9.8 \%$ of participants were either seeing or waiting to see a mental health professional (respectively), while just over half of participants (55.2\%) were not seeing a mental health professional. Of those participants who were currently accessing support, $20.3 \%$ waited one week or less to see their mental health professional, $39.5 \%$ waited between $2-5$ weeks, $13.8 \%$ waited 6-8 weeks, and $15 \%$ waited three months or more. 


\section{Sources of support}

Participants had accessed support from a range of sources, most commonly friends (84.7\%), followed by counsellors (73.3\%), GPs or doctors (69.6\%), school counsellors (54.4\%), whānau (51\%), and psychologists (49\%).

\section{Reasons for accessing support}

The most common reasons for accessing support were due to feeling depressed $(86.8 \%)$ or anxious $(81.7 \%)$, followed by general life stress $(60.5 \%)$, relationships (48.3\%), and trauma (44.2\%), however participants also accessed support directly related to their rainbow identity, such as support around sexuality $(29.1 \%)$ and gender $(17.6 \%)$.

\section{Helpfulness of support}

Participants were invited to think about all the mental health professionals they had seen, and rate on a 5-point Likert scale how helpful they had been in supporting their mental health overall $(1=$ Extremely unhelpful to $5=$ Extremely helpful $)$. Participants rated the average overall helpfulness of mental health professionals as 3.26 $(S D=1.03)$. Slightly over half of participants found mental health professionals "Mostly helpful" (45.9\%) or "Extremely helpful" (6.4\%), 19.5\% found them "Neither helpful nor unhelpful" overall, and a little under a third of participants found mental health professionals "Mostly unhelpful" (23.6\%) or "Extremely unhelpful” (4.6\%).

Participants were asked to rate how helpful each source of support had been in supporting their mental health on a 5-point Likert scale $(1=$ Extremely unhelpful to $5=$ Extremely helpful). Of the different types of mental health professionals, psychologists were rated as most helpful overall $(M=3.49, S D=1.09)$, while school counsellors were rated the least helpful $(M=2.63, S D=1.27)$.

\section{Rainbow-friendly services and identity centrality}

The vast majority of participants $(95.4 \%)$ reported that it was important to them that mental health services are rainbow-friendly $(M=4.82, S D=.60)$, however participants were less likely to agree that they knew where to find queer, trans, and intersex-friendly services $(M=2.91, S D=1.26)$. 


\section{Comfort in mental health settings}

Participants were asked how comfortable they would feel telling a new mental health professional about their sexuality, about their gender (for trans/nonbinary participants), that they are intersex (for intersex participants), and telling them about something else (participants were invited to specify if relevant or skip the question if not). Participants were also asked how comfortable they would feel discussing issues related to sexuality, gender, and being intersex (for intersex participants). All responses were measured on a 5-point Likert scale $(1=$ Extremely uncomfortable to $5=$ Extremely comfortable). Responses to these items are reported in Figure 10. Participants' scores on the items about discussing issues related to gender and discussing issues related to sexuality were added together to create a "combined comfort discussing gender and sexuality" variable. 206 participants wrote in a response to "telling them about something else." The most common response was telling mental health professionals about experiences of abuse and trauma $(n=56)$, followed by mental health or mental illness $(n=38)$, and polyamory or non-monogamy $(n=27)$.

\section{Discrimination experiences}

Participants were asked to rate the extent to which they agreed or disagreed that they had had negative experiences with other health professionals in the past, had experienced discrimination outside of mental health settings on the basis of their sex, sexuality, and/or gender, ${ }^{64}$ and had heard stories of other queer, trans, and intersex folks having negative experiences with mental health professionals. Almost half (45.1\%) of participants reported that they had experienced discrimination outside of mental health settings, and $21.5 \%$ reported that they had had negative experiences with other health professionals in the past. More than a third (35.8\%) reported that they heard stories of other queer, trans, and intersex folks having negative experiences with mental health professionals.

\footnotetext{
${ }^{64}$ At the time of surveying I was using the umbrella term 'sex, sexuality, and gender diverse' rather than 'sexuality, gender, and sex characteristic diverse'. I changed my terminology because I thought it was unclear whether 'sex' referred to sexual activity or sex characteristics. At points in this chapter I use my former terminology to accurately reflect what participants were asked.
} 


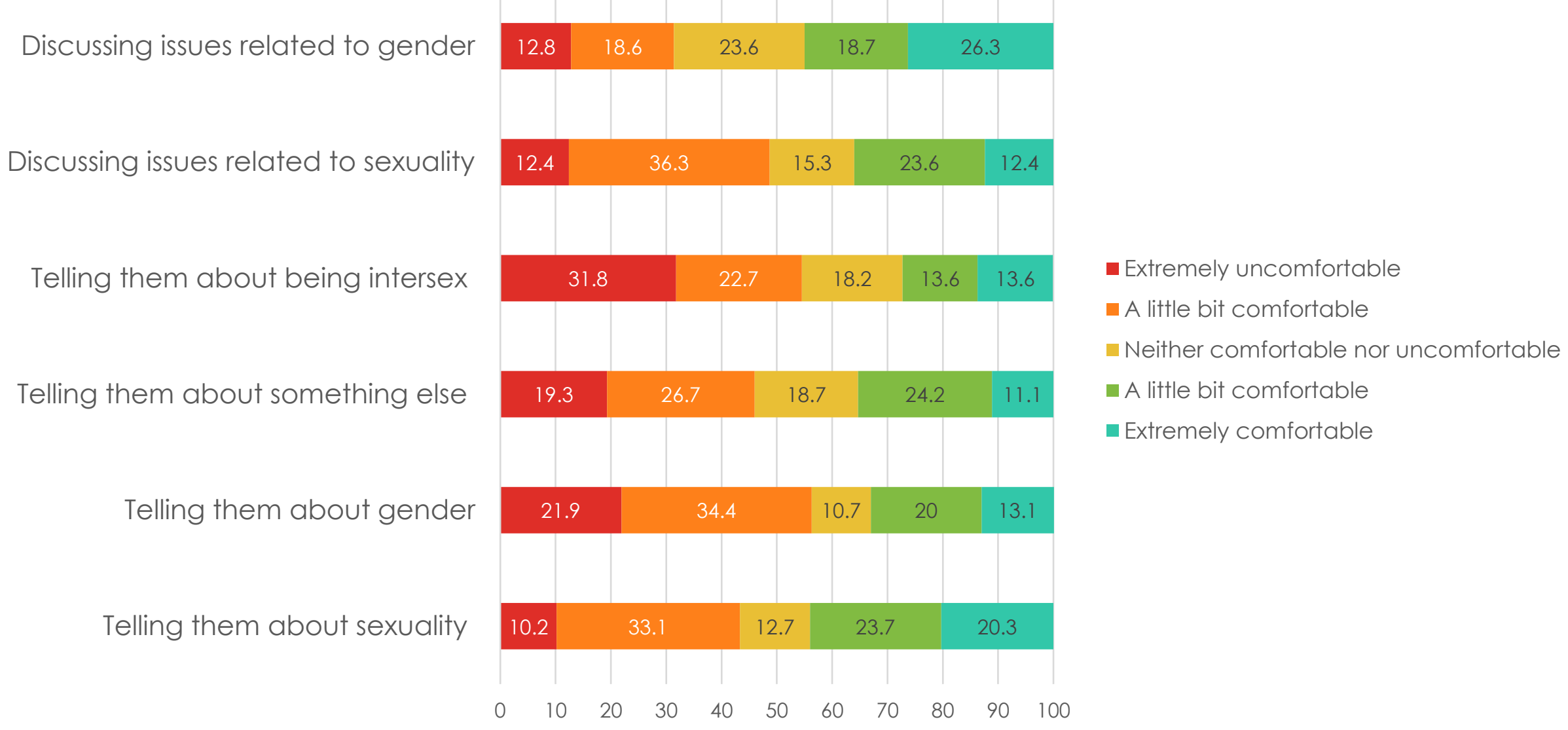

Figure 10. Comfort when meeting new mental health professionals. 


\section{Safety signals}

To assess the helpfulness of safety signals, participants were asked how helpful it would be if a new mental health professional took a series of actions, e.g. "shared their pronouns when introducing themselves" and "used inclusive language that didn't assume your identity". Participants were then asked to rate how helpful each would be. Responses were measured on a 5-point Likert scale $(1=$ Extremely unhelpful to $5=$ Extremely helpful) and are presented in Figure 11.

\section{Positive and negative experiences of accessing mental health support}

Participants were presented with a randomly ordered list of 23 possible experiences and asked to select those they had experienced when accessing support from a mental health professional. In later analyses I coded 12 of these experiences as positive (e.g. "the mental health professional affirmed and validated your identity") and 13 as negative (e.g. "the mental health professional made insensitive or hurtful comments about your identity"), though they were not labelled as positive and negative for participants. ${ }^{65}$ Participants were invited to share other negative or positive experiences in open text boxes. Participants that were currently seeing a mental health professional or had seen a professional in the last few years were specifically asked to think about experiences they had had within the last five years. Those that had last seen a mental health professional more than five years previously were asked to think about whether the experiences had happened at any time. Figures 12 and 13 present an overview of participants' positive and negative experiences when accessing mental health support, respectively. Participants' total positive experiences scores were added together to create a combined positive experiences variable and participants' total negative experiences scores were added together to create a "combined negative experiences" variable. ${ }^{66}$

\footnotetext{
${ }^{65}$ Items were coded as 'positive' if they were positively associated with the overall helpfulness item, and 'negative' if they were negatively associated with the overall helpfulness item. All items were significantly associated with the overall helpfulness item in the direction I expected, except the item "asked about the relationship between identity and mental health", which was not associated with overall helpfulness. I coded this as a 'positive' experience based on my interview data, which suggested this is a helpful question to ask in mental health settings.

${ }^{66}$ Combined variables did not include the open-ended 'other positive' or 'other negative' experiences.
} 


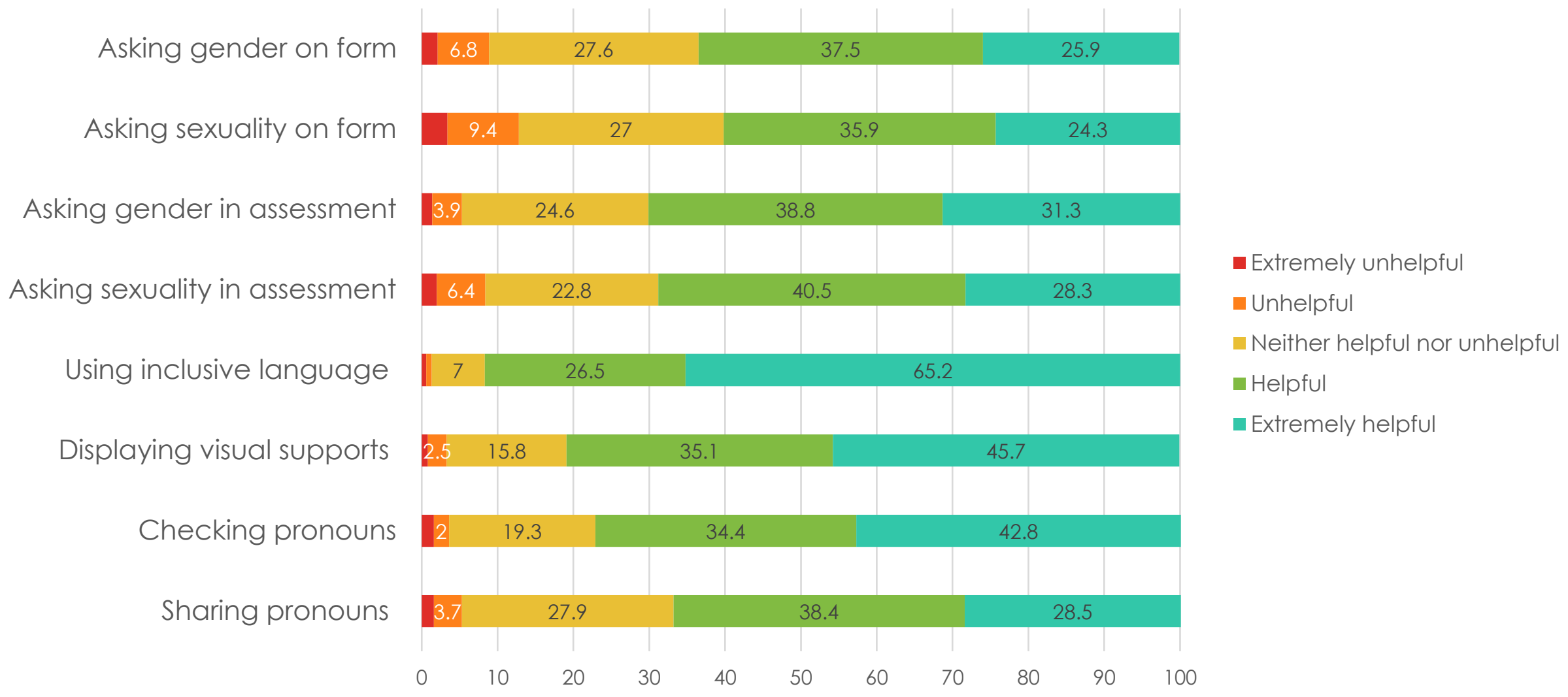

Figure 11. Helpfulness of safety signals. 


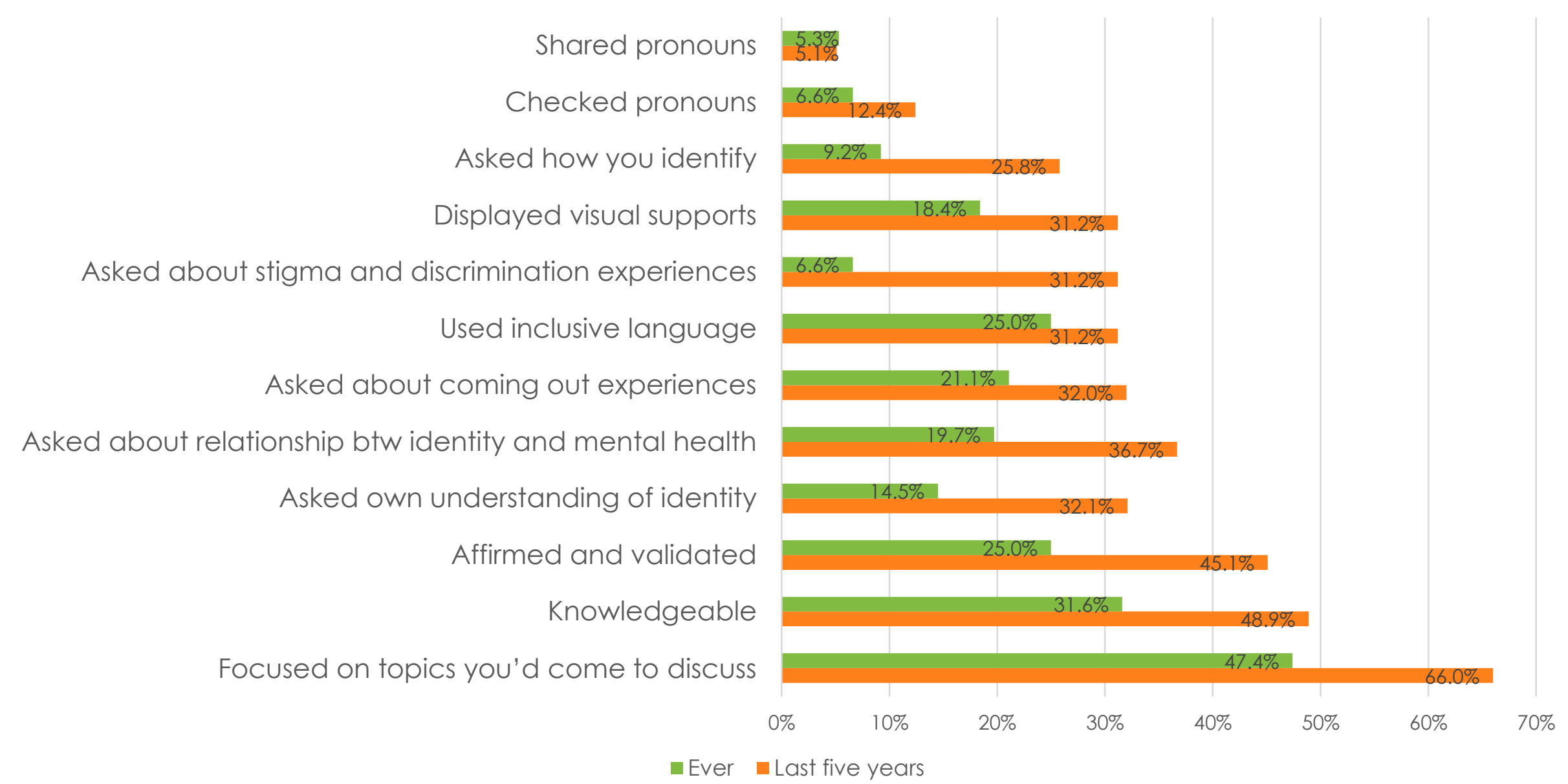

Figure 12. Positive experiences when accessing mental health support. 
Refused to see you after you came out

Tried to change your identity

Refused to talk about your identity when wanted

Used your deadname

Implied your identity was a phase or not real

Made insensitive or hurtful comments about identity

Implied your identity was caused by past trauma

Misgendered you

Blamed your difficulties on your identity

Focused on identity when it was not the issue

Seemed surprised or uncomfortable when you came out

Required education

Assumed you were straight or cis
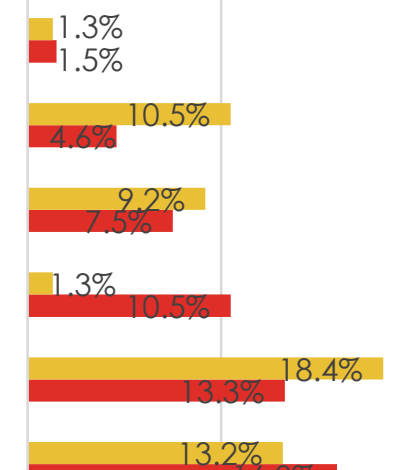

$13.2 \%$
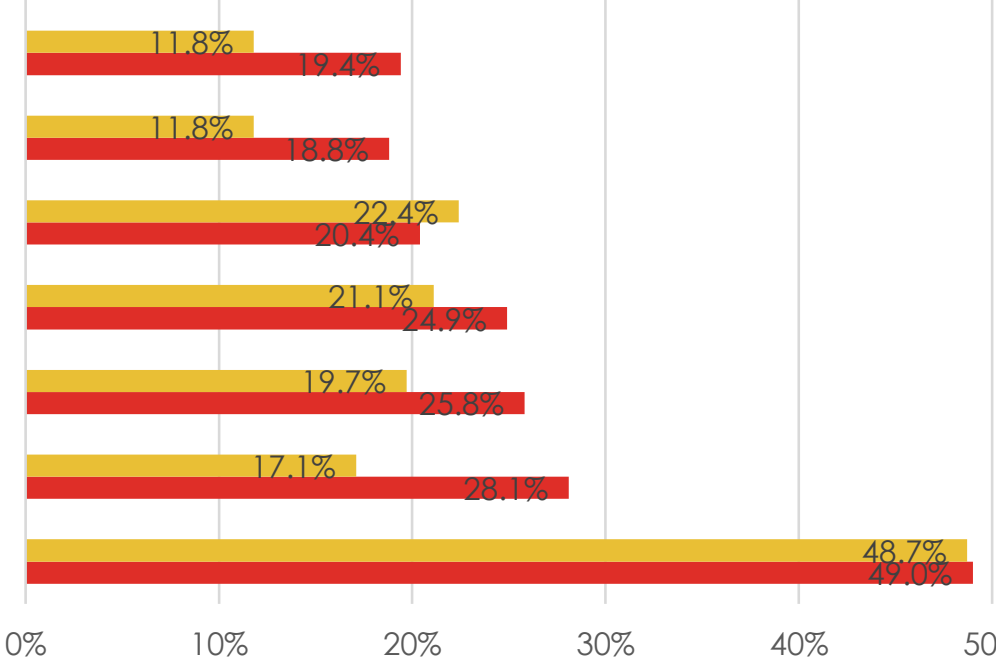

Figure 13. Negative experiences when accessing mental health support. 
One item on the list of possible experiences was "the mental health professional required education about sex, sexuality, and/or gender". Participants who selected this item were then asked to select what their mental health professional required education about, e.g. language and terminology, pronoun use, and the concept of minority stress. Of the 353 participants who reported a past experience of a mental health professional requiring education about sex, sexuality, and/or gender, 266 (75.4\%) reported they had required education about language and terminology, 159 (45\%) about pronoun use, 229 (64.9\%) about the nature of sex, sexuality, and/or gender, $189(53.5 \%)$ about the concept of minority stress, 229 (64.9\%) about issues faced by sex, sexuality, and gender diverse people, and 4 about what it means to be intersex $(66.7 \%$ of the intersex people who responded to this question). A further 41 people (18.6\%) noted something else that their mental health professional had required education about, including privilege, feminism, disability, gender dysphoria, medical transition, non-monogamy, kink, the Treaty of Waitangi, bias in the medical community, and queer relationships.

\section{Worries when meeting a new mental health professional}

Participants were asked to rate the extent to which they agreed or disagreed that they worry a new mental health professional will respond awkwardly or with discomfort when they come out, will discriminate against them on the basis of sex, sexuality and/or gender, and will discriminate against them on the basis of ethnicity. Participants were also asked to rate the extent to which they agreed or disagreed that they assume new mental health professionals will lack knowledge about sex, sexuality and gender diversity. Responses are presented in Figure 14. Participant scores on these four items were added together to create a combined worries variable. 
I worry they will discriminate against me on the basis of my ethnicity

I worry they will discriminate against me on the basis of my sex, sexuality, and/or gender

I worry they will respond awkwardly or with discomfort when I come out

I assume they will lack knowledge about sex, sexuality, and gender diversity
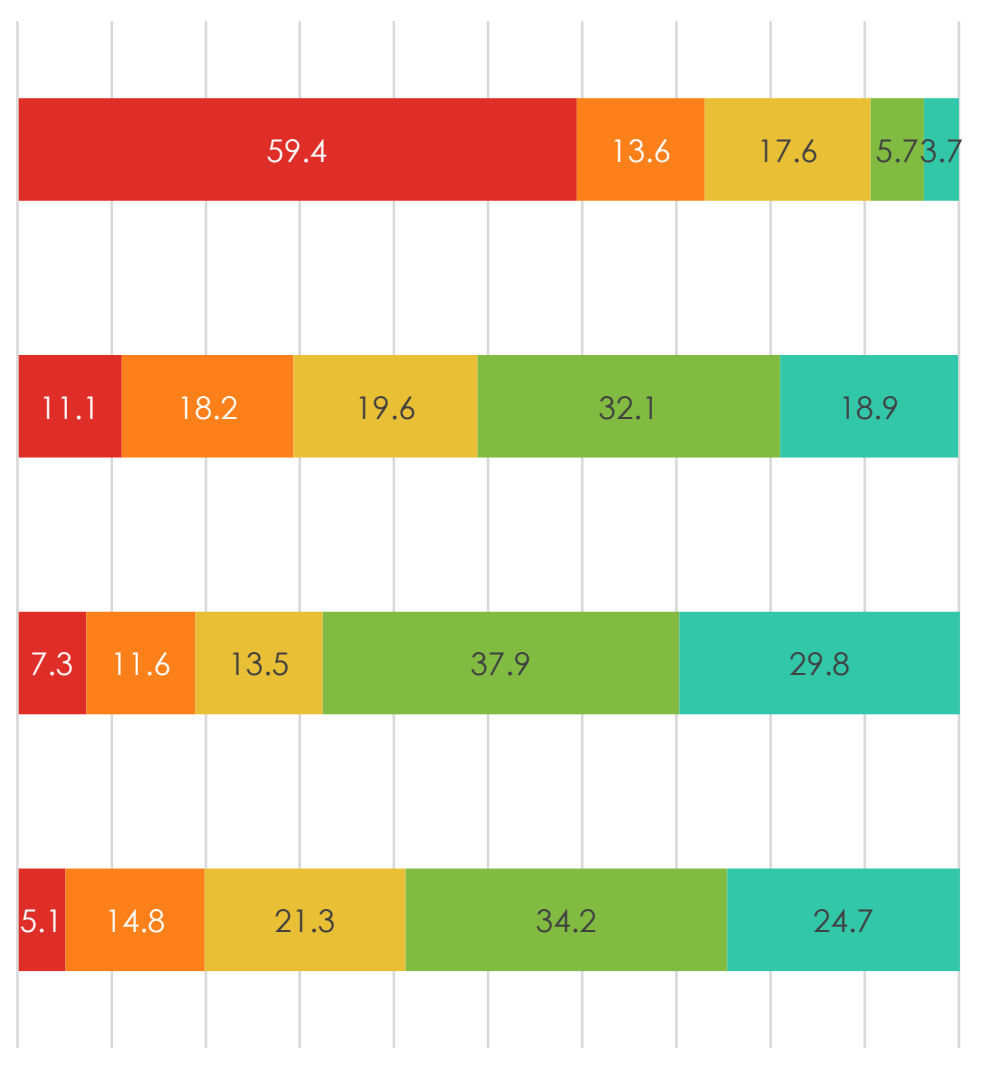

- Strongly disagree

- Slightly disagree

Neither agree or disagree

aslightly agree

- Strongly agree

Figure 14. Worries in mental health settings. 


\section{Importance of knowing identity}

Participants were asked to rate how important it is to them that mental health professionals know their sexual orientation, that they are trans or nonbinary (for trans/nonbinary participants), and that they are intersex (for intersex participants). Responses were measured on a 4-point Likert scale ( $1=$ Not at all important, $2=$ A little bit important, 3 = Extremely important, 4 = It depends why I'm seeing them), and are presented in Figure 15.

$$
\begin{aligned}
& \text { Importance of knowing you are } \\
& \text { intersex }
\end{aligned}
$$

Importance of knowing you are trans or nonbinary

Importance of knowing sexuality

- Not at all important

Extremely important

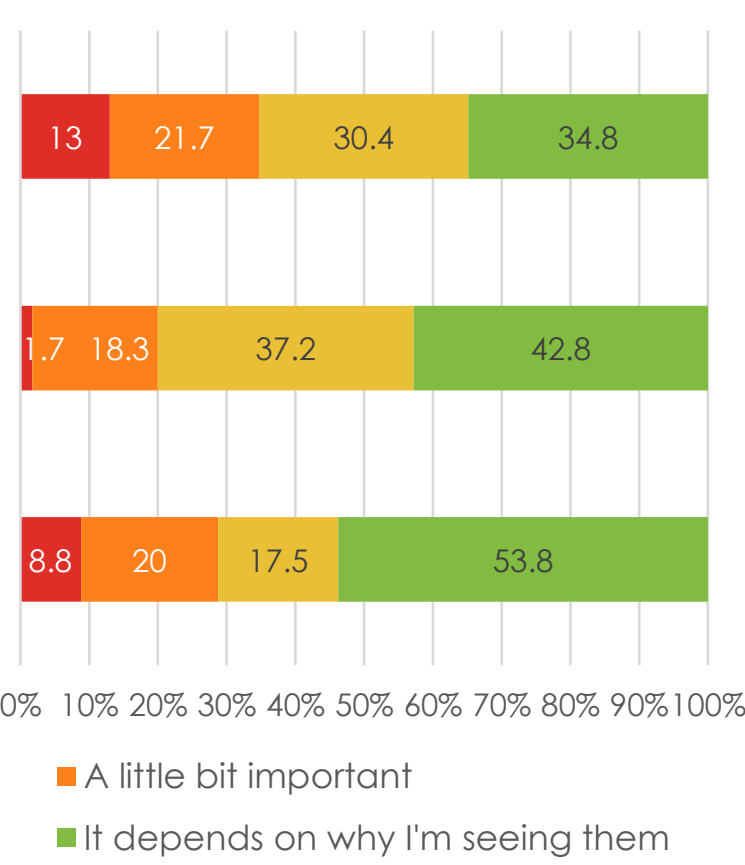

Figure 15. Importance of identity in mental health settings.

\section{Experiences of Accessing Gender-Affirming Healthcare}

\section{Gender-affirming healthcare access}

Participants who had accessed gender-affirming healthcare, or were in the process of doing so, were asked to specify which services. Responses are shown in Table 2. Participants who had reported that they wanted to access gender-affirming healthcare but had not done so yet were invited to select one or more possible reasons for this (e.g. "I'm still figuring out what healthcare I need"). The most common reasons 
for not accessing gender-affirming healthcare were because participants were nervous about the process $(61.9 \%)$, still figuring out what healthcare they needed $(59.7 \%)$ or were unsure how to go about accessing gender-affirming healthcare (59.7\%).

Of the 228 participants who reported how they had accessed gender-affirming healthcare, $132(57.9 \%)$ had accessed this through the public health system, 18 (7.9\%) through private health services, and 78 (34.2) through a mix of public and private services. Of those who had accessed gender-affirming healthcare privately, 93 shared their reasons for doing so: Twenty-five (26.9\%) reported the healthcare they needed was not available where they lived, 52 (55.9\%) reported that the waiting times are too long in the public system, 21 (22.6\%) reported they were denied care in the public system, 25 (26.9\%) reported they believed the quality of care is higher in the private than public system, and $30(32.3 \%)$ provided another reason.

Table 2

Access to gender-affirming healthcare services

\begin{tabular}{lcc}
\hline Item & $\begin{array}{c}\text { Has accessed } \\
n(\%)\end{array}$ & $\begin{array}{c}\text { In process of accessing } \\
n(\%)\end{array}$ \\
\hline Hormone therapy & $133(90.5)$ & $25(32.1)$ \\
Top surgery & $30(20.4)$ & $19(24.4)$ \\
Hormone/puberty blockers & $9(6.1)$ & - \\
Gender therapy & $9(6.1)$ & $1(1.3)$ \\
Voice training/therapy & $8(5.4)$ & - \\
Hysterectomy & $6(4.1)$ & $4(5.1)$ \\
Genital surgery NFD & $5(3.4)$ & $12(15.4)$ \\
Orchiectomy & $2(1.4)$ & $2(2.6)$ \\
Hair removal & $2(1.4)$ & - \\
Facial feminization surgery & $2(1.4)$ & $2(2.6)$ \\
Surgery NFD & $2(1.4)$ & - \\
Vocal surgery & $1(.7)$ & - \\
Oophorectomy & $1(.7)$ & $2(2.6)$ \\
Fertility help/preservation & - & - \\
\hline
\end{tabular}

Note. In total, 147 participants reported they had accessed gender-affirming care and 78 reported they were in the process of accessing gender-affirming care. 
The majority of participants who had accessed gender-affirming healthcare $(n=$ $187,81.3 \%$ ) had not moved to a different town or city in order to do so. Sixteen participants (7.0\%) had moved to a different town or city in order to access genderaffirming healthcare, $22(9.6 \%)$ had not moved specifically for this reason, but noted it was a factor in their decision to move, and five participants (2.2\%) reported having used someone else's address and travelling to their DHB for care.

\section{Waiting time for gender-affirming healthcare}

Participants were asked how long it took to receive each gender-affirming healthcare service. Of the 113 participants who provided a response regarding wait times for hormone therapy, the mean waiting time was 46.7 weeks $(S D=48.08$, range $=$ 0-260 weeks). I was unable to estimate the average waiting time for other genderaffirming services as most participants only provided a response about hormone therapy (the example used in the question).

\section{Mental health assessments for gender-affirming healthcare}

Participants who had accessed gender-affirming healthcare or were in the process of doing so were asked about seeing a mental health professional for an assessment to access care. Of the 229 participants that responded to this question, 169 $(73.8 \%)$ had seen a mental health professional for an assessment, $16(7 \%)$ were waiting to see a mental health professional, $37(16.2 \%)$ had accessed care without an assessment, and 7 (3.1\%) had an assessment with someone other than a mental health professional (e.g. a GP or other doctor). Of the 176 participants who had had an assessment in order to access gender-affirming healthcare, 10 participants (5.7\%) last had an assessment more than ten years ago, 17 (9.7\%) between five and ten years ago, 65 (36.9\%) between one and five years ago, 63 (35.8) within the last year, and 21 $(11.9 \%)$ were seeing a mental health professional to access gender-affirming care at the time of the survey.

Participants who had seen a mental health professional or other professional for an assessment to access gender-affirming healthcare were presented with a list of possible experiences and asked to select those they had experienced during their mental health assessment to access gender-affirming healthcare. Responses are shown in Figure 16. 
Asked what support you have from others

Asked whether you understood the physical effects of your healthcare

Asked how long you have been presenting as your gender

Assumed you wanted to conform to binary gender norms

Asked what dothes you wore as a child

Focussed on Western/Pākehā ideas about gender

Asked what toys you played with as a child

Asked questions about your sexuality and/or sex life

Gave you a questionnaire about dysphoria

Questioned the validity of your gender

Said you had to have a period of real life experience

Implied your gender was caus ed by past trauma

Implied your transition should include every medical option

Implied your gender was a phase

Another neg ative experience

Another positive exper ience
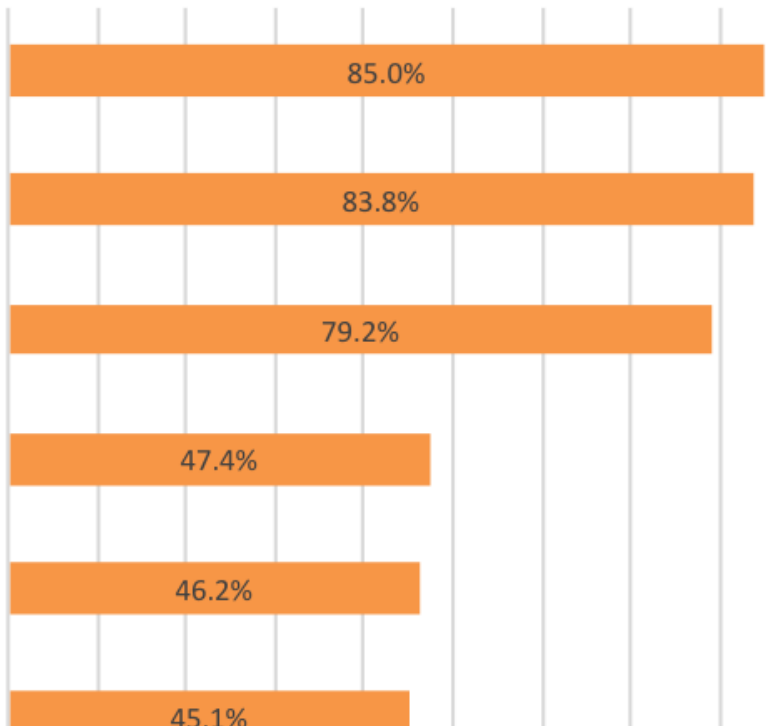

$45.1 \%$
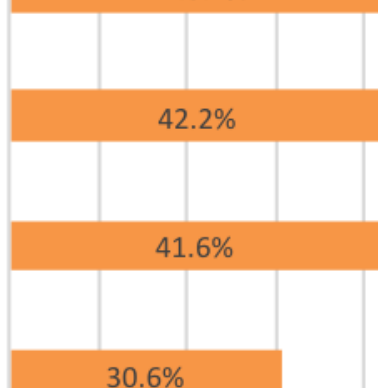

$30.6 \%$

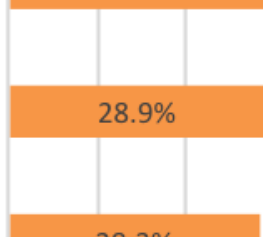

$28.3 \%$

\section{$20.2 \%$}

$16.80 \%$

$13.3 \%$

$13.9 \%$

$11.0 \%$

$\begin{array}{llllllllll}0 \% & 10 \% & 20 \% & 30 \% & 40 \% & 50 \% & 60 \% & 70 \% & 80 \% & 90 \%\end{array}$

Figure 16. Experiences during assessments to access gender-affirming healthcare. 
Participants who had seen a mental health professional or other professional for an assessment to access gender-affirming healthcare were then presented with a list of statements and asked to what extent they agreed or disagreed with each statement. Responses are shown in Figure 17.

Finally, participants who had seen a mental health professional or other professional for an assessment to access gender-affirming healthcare were asked about their views on the aims of the assessment. Of the 175 participants that responded to this question, 110 (62.9\%) reported the assessment was conducted "to check I was able to make decisions about my healthcare", 121 (69.14\%) "to make sure I was 'really trans' or 'trans enough'”, 56 (32\%) "to put support systems in place while I accessed genderaffirming healthcare", 116 (66.3\%) "to check I understood what would happen to my body", and $35(20 \%)$ reported another aim. The most common open-ended response about the aims of assessment were to ensure mental health difficulties were well managed prior to transition, or to ensure participants were not too unwell to access care $(n=13,34.2 \%)$.

\section{Denial of gender-affirming healthcare}

Of the participants that had accessed, or were in the process of accessing, gender-affirming healthcare, 165 (73.3\%) reported that they had not been denied care, and $60(26.7 \%)$ reported that they had been denied care they had tried to access. Slightly under half of participants who had been denied care had been told they needed to be more mentally well to access care $(n=29,49.2 \%)$. The next most common reason was length of time ( $n=23,39 \%)$, followed by high BMI or weight $(n=8,13.6 \%)$ and lack of support $(n=4,6.8 \%)$. Twenty-seven participants $(45.8 \%)$ provided another reason for being denied care, including the mental health professional believing participants were not trans $(n=5)$ or that they would change their mind later $(n=4)$, being denied due to age $(n=4)$, due to lack of funding or non-existent services $(n=3)$, or due to health professionals lacking knowledge about gender-affirming healthcare or not believing it should be provided $(n=3)$. 
I felt pressure to present my gender in a way that conformed to Western/Pākehā ideas

I felt pressure to present as hyper feminine or hyper masculine during my assessment

I felt pressure to conform to a dominant narrative during my assessment

The person doing the assessment had a good understanding of gender diversity and gender dysphoria

The person doing the assessment respected and validated my gender

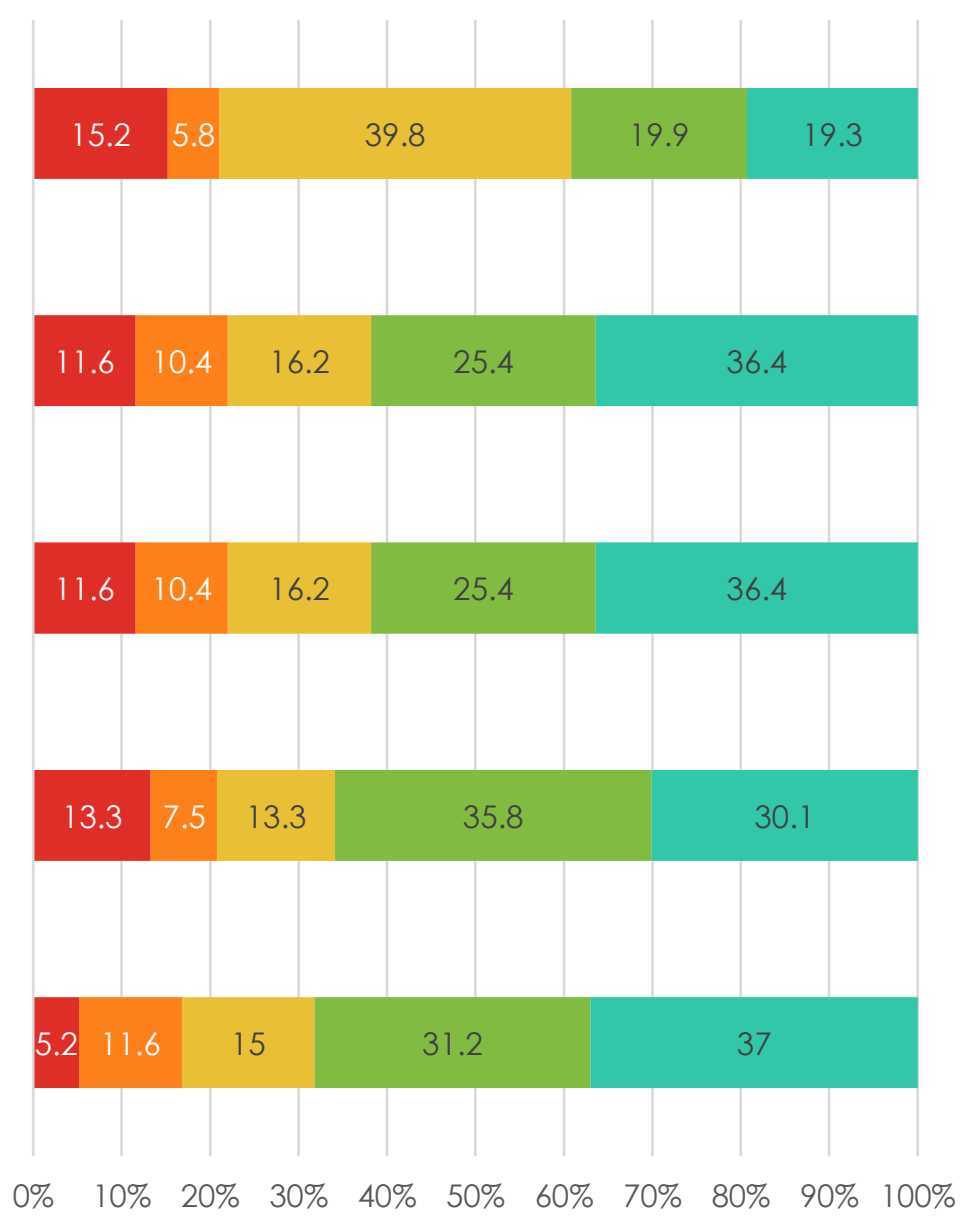

- Strongly disagree

- Slightly disagree

- Neither agree nor disagree

- Slightly agree

- Strongly agree

Figure 17. Respect, knowledge, and the trans narrative in assessments for gender-affirming healthcare. 


\section{Inferential Analyses}

Given the large number of questions in the survey, I could have conducted a multitude of further analyses using different combinations of variables related to my research questions. Due the space constraints in this thesis, I am only able to report a select few further analyses. Following the completion of this thesis, I plan to conduct (and publish) some of the analyses I was unable to include here.

\section{Demographic Differences on Select Survey Items}

First, I conducted a series of MANOVAs (Multivariate Analysis of Variance) examining potential differences on a select number of variables based on sexual orientation, gender, trans status, intersex status, age, location, ethnicity, and intersecting identities. ${ }^{67}$ I chose dependent variables that provided a broad overview of survey data: participants' self-rated mental health, ease of access to mental health services, combined worries when accessing support, combined comfort discussing sexuality and gender in mental health settings, and total number of positive and negative experiences when accessing mental health support.

There was a statistically significant difference in dependent variables based on sexual orientation $\left(F(28,4433)=3.22, p<.001\right.$; Wilk's $\Lambda=.93$, partial $\left.\eta^{2}=.02\right)$, gender $\left(F(35,5177)=13.18, p<.001 ;\right.$ Wilk's $\Lambda=.70$, partial $\left.\eta^{2}=.07\right)$, trans status $(F(14,2458)$ $=31.01, p<.001 ;$ Wilk's $\Lambda=.72$, partial $\left.\eta^{2}=.15\right)$, intersex $\operatorname{status}^{68}(F(14,2474)=7.85$, $p<.001 ;$ Wilk's $\Lambda=.92$, partial $\left.\eta^{2}=.03\right)$, age $(F(28,4461)=6.75, p<.001 ;$ Wilk's $\Lambda=$ .86 , partial $\left.\eta^{2}=.04\right)$, ethnicity $\left(F(28,4454)=2.54, p<.001 ;\right.$ Wilk's $\Lambda=.95$, partial $\eta^{2}=$ $.01)$, socioeconomic status $\left(\operatorname{SES} ; F(7,1240)=8.92, p<.001 ;\right.$ Wilk's $\Lambda=.95$, partial $\eta^{2}$ $=.05)$, and being neurodiverse $\left(F(7,1240)=15.20, p<.001\right.$; Wilk's $\Lambda=.92$, partial $\eta^{2}=$ $.08)$, non-monogamous $\left(F(7,1240)=2.31, p=.02\right.$; Wilk's $\Lambda=.99$, partial $\left.\eta^{2}=.01\right)$, or physically disabled $\left(F(7,1240)=8.55, p<.001\right.$; Wilk's $\Lambda=.95$, partial $\left.\eta^{2}=.05\right)$. There

\footnotetext{
${ }^{67}$ I conducted analyses of group differences on intersecting identities endorsed by at least $5 \%$ of the sample, as I was unlikely to detect differences in the remaining groups due to a lack of statistical power. This means conducting analyses of group differences based on whether participants described themselves as low SES, faith/religious, migrants, neurodiverse, non-monogamous, and physically disabled.

${ }^{68}$ For the purposes of this analysis, participants that noted they did not understand the question about being intersex were coded as not intersex.
} 
was no statistically significant difference in dependent variables based on location $\left(F(28,4425)=1.46, p=.06 ;\right.$ Wilk's $\Lambda=.97$, partial $\left.\eta^{2}=.01\right)$, or being religious $\left(F(7,1240)=2.02, p=.05 ;\right.$ Wilk's $\Lambda=.99$, partial $\left.\eta^{2}=.01\right)$ or a migrant $(F(7,1240)=$ $1.14, p=.33 ;$ Wilk's $\Lambda=.99$, partial $\left.\eta^{2}=.01\right)$.

Tables presenting mean scores on each dependent variable and significant differences between mean scores based on sexual orientation, gender, trans and intersex status, age, location, ethnicity, and other important identities are shown in Appendix K. In brief, self-rated mental health was particularly low among asexual and multiple gender attracted participants, as well as trans participants (especially trans men and nonbinary participants) and those unsure if they were trans, intersex participants and those unsure if they were intersex, younger participants, and participants who described themselves as low SES, neurodiverse, and physically disabled. Asexual and queer participants and participants whose sexual orientation was coded as "something else" reported higher levels of worry in mental health settings than gay/lesbian participants and lower levels of comfort discussing gender and sexuality. Trans and cis participants' mean scores differed on all dependent variables, except their overall helpfulness ratings. Participants tended to be less comfortable discussing gender and sexuality if they were aged 14 or 15 (compared to 25 and older), of low SES, neurodiverse, or physically disabled.

There were few group differences in ease of accessing support, although trans and intersex participants and those unsure if they were intersex had a lower mean score than cis and non-intersex participants, respectively. Participants also found it more difficult to access support if they were neurodiverse, physically disabled, or low SES. There were few group differences in the number of positive experiences participants reported, with the exception of gender differences (trans women, trans men, and nonbinary people had the highest positive experience scores). Additionally, neurodiverse participants reported fewer positive experiences than participants who were not neurodiverse, and 25-54-year-olds reported fewer positive experiences than 16-24-year-olds.

With regard to ethnicity, sole-identified Māori and Māori who identified with ethnicities other than Pākehā had higher worry scores than all other ethnicity groups. Both groups also had lower overall helpfulness scores than sole-identified Pākehā and 
participants who identified with ethnicities other than Pākehā and Māori. Participants in the Pākehā and other ethnicities group also reported that it was more difficult to access support than participants in the Other ethnicity group.

\section{Past Experiences, Worries, and Comfort}

To explore whether past experiences in mental health settings were associated with future worries and comfort in mental health settings I conducted a series of bivariate correlations. Here I examined experiences that had happened in the last five years, rather than more than five years ago, due to the larger sample size for more recent experiences $(n=1211)$ than more distant experiences $(n=76)$. Bivariate correlations between positive experiences and comfort/worries are presented in Table 3, and between negative experiences and comfort/worries in Table $4 .{ }^{69}$

As seen in Table 3, past negative experiences were generally negatively associated with future comfort sharing about and discussing issues related to gender and sexuality. Conversely, Table 4 shows that past positive experiences were generally positively associated with participants' future comfort sharing about, and discussing issues related to, gender and sexuality. The opposite patterns were seen with past experiences and future worries: past positive experiences tended to be negatively associated with future worries, and past negative experiences tended to be positively associated with future worries. Exceptions to this was seen in the item "asked about the relationship between identity and mental health", which was positively associated with future worries about professional discomfort and discrimination.

${ }^{69}$ Because multiple comparisons increases the family-wise error rate, I set a conservative criterion for significance of $p<.01$. 
Table 3

Bivariate correlations between negative experiences and comfort/worries

\begin{tabular}{|c|c|c|c|c|c|c|c|}
\hline & $\begin{array}{l}\text { Comfort } \\
\text { sharing } \\
\text { sexuality }\end{array}$ & $\begin{array}{c}\text { Comfort } \\
\text { sharing } \\
\text { gender }\end{array}$ & $\begin{array}{c}\text { Comfort } \\
\text { discussing } \\
\text { sexuality } \\
\text { issues }\end{array}$ & $\begin{array}{l}\text { Comfort } \\
\text { discussing } \\
\text { gender } \\
\text { issues }\end{array}$ & $\begin{array}{l}\text { Worry about } \\
\text { professional } \\
\text { lacking } \\
\text { knowledge }\end{array}$ & $\begin{array}{l}\text { Worry about } \\
\text { professional } \\
\text { discomfort }\end{array}$ & $\begin{array}{c}\text { Worry about } \\
\text { discrimination } \\
\text { from } \\
\text { professional }\end{array}$ \\
\hline Assumed you were straight or cis & $-.10^{*}$ & -.13 & $-.14 *$ & $-.14^{*}$ & $.22 *$ & $.17 *$ & $.18^{*}$ \\
\hline Required education & -.01 & .03 & -.07 & $-.15 *$ & $.31^{*}$ & $.18^{*}$ & $.15^{*}$ \\
\hline $\begin{array}{l}\text { Seemed surprised or uncomfortable when you came } \\
\text { out }\end{array}$ & $-.10 *$ & $-.19 *$ & $-.13 *$ & $-.16^{*}$ & $.21 *$ & $.25^{*}$ & $.24 *$ \\
\hline Focused on identity when it was not the issue & -.02 & -.07 & -.07 & $-.12 *$ & $.16^{*}$ & $.15^{*}$ & $.13 *$ \\
\hline Blamed your difficulties on your identity & -.02 & .01 & -.04 & $-.08 *$ & $.18^{*}$ & $.15^{*}$ & $.21 *$ \\
\hline Misgendered you & -.04 & -.10 & $-.08 *$ & $-.27 *$ & $.23 *$ & $.19 *$ & $.24 *$ \\
\hline Implied your identity was caused by past trauma & -.07 & -.06 & -.07 & $-.15^{*}$ & $.15^{*}$ & $.16^{*}$ & $.23 *$ \\
\hline Made insensitive or hurtful comments about identity & $-.10 *$ & -.13 & $-.14^{*}$ & $-.19 *$ & $.22 *$ & $.20 *$ & $.22 *$ \\
\hline Implied your identity was a phase or not real & $-.10 *$ & -.12 & $-.10^{*}$ & $-.14 *$ & $.10^{*}$ & $.14^{*}$ & $.18^{*}$ \\
\hline Used your deadname & -.05 & -.02 & $-.08 *$ & $-.15^{*}$ & $.18^{*}$ & $.16^{*}$ & $.21 *$ \\
\hline $\begin{array}{l}\text { Refused to talk about your identity when you } \\
\text { wanted to discuss it }\end{array}$ & -.05 & -.02 & $-.09 *$ & $-.15^{*}$ & $.12 *$ & $.14^{*}$ & $.17 *$ \\
\hline Tried to change your identity & $-.08 *$ & -.08 & $-.09 *$ & -.07 & $.08 *$ & .07 & $.12^{*}$ \\
\hline Refused to see you after you came out & -.07 & -.04 & -.04 & -.04 & .06 & .04 & .06 \\
\hline
\end{tabular}

Note. $*$ significant difference at the 0.01 level. 
Table 4

Bivariate correlations between positive experiences and comfort/worries

\begin{tabular}{|c|c|c|c|c|c|c|c|}
\hline & $\begin{array}{c}\text { Comfort } \\
\text { sharing } \\
\text { sexuality }\end{array}$ & $\begin{array}{l}\text { Comfort } \\
\text { sharing } \\
\text { gender }\end{array}$ & $\begin{array}{c}\text { Comfort } \\
\text { discussing } \\
\text { sexuality } \\
\text { issues }\end{array}$ & $\begin{array}{l}\text { Comfort } \\
\text { discussing } \\
\text { gender } \\
\text { issues }\end{array}$ & $\begin{array}{c}\text { Assume } \\
\text { professional } \\
\text { will lack } \\
\text { knowledge }\end{array}$ & $\begin{array}{c}\text { Worry about } \\
\text { professional } \\
\text { discomfort }\end{array}$ & $\begin{array}{l}\text { Worry about } \\
\text { discrimination } \\
\text { from } \\
\text { professional }\end{array}$ \\
\hline $\begin{array}{l}\text { Focused on topics you'd come to } \\
\text { discuss }\end{array}$ & .08 & .07 & .09 & .02 & $-.09 *$ & -.07 & -.12 \\
\hline Knowledgeable & $.13 *$ & .06 & $.17 *$ & $.08 *$ & $-.19 *$ & $-.14 *$ & $-.11 *$ \\
\hline Affirmed and validated & $.11 *$ & $.16^{*}$ & $.15^{*}$ & .06 & -.04 & -.05 & $-.11 *$ \\
\hline Asked own understanding of identity & .07 & $.17 *$ & .05 & .07 & $.17 *$ & .05 & -.07 \\
\hline $\begin{array}{l}\text { Asked about relationship between } \\
\text { identity and mental health }\end{array}$ & .01 & .08 & .03 & -.05 & .04 & $.10 *$ & $.08 *$ \\
\hline Asked about coming out experiences & $.09 *$ & .07 & $.14 *$ & -.01 & -.02 & -.01 & -.01 \\
\hline Used inclusive language & $.08 *$ & .07 & $.12 *$ & $.08 *$ & $-.09 *$ & $-.09 *$ & -.06 \\
\hline $\begin{array}{l}\text { Asked about stigma and } \\
\text { discrimination experiences }\end{array}$ & .06 & .05 & $.08 *$ & -.04 & -.01 & .02 & .04 \\
\hline Displayed visual supports & .06 & .12 & $.09 *$ & .01 & -.07 & -.05 & -.07 \\
\hline Asked how you identify & $.08 *$ & .12 & $.08 *$ & -.02 & -.03 & -.03 & -.04 \\
\hline Checked pronouns & .00 & .11 & .04 & -.03 & .03 & .03 & .03 \\
\hline Shared pronouns & .03 & .03 & .04 & .02 & -.03 & -.01 & .00 \\
\hline
\end{tabular}

Note. * significant difference at the 0.01 level. 
Tables 3 and 4 show that past negative experiences were more often associated with future comfort and worries than past positive experiences. Associations between past negative experiences and future comfort and worries also tended to be stronger than associations between past positive experiences and future comfort and worries. For example, the strongest correlation across Tables 3 and 4 was that between the past experience "seemed surprised or uncomfortable when you came out" and future worry that mental health professionals will respond to coming out awkwardly or with discomfort. All correlations, however, were weak or moderate. Finally, it is interesting to note that past experiences of sharing and checking pronouns and "refused to see you after you came out" was not associated with future worries and comfort, and that comfort sharing gender was associated with only three of the 25 past experiences; fewer significant relationships than any of the other comfort or worry variables.

\section{Past Experiences and Overall Helpfulness}

To better understand the impact of past positive experiences, I conducted a multiple regression using the 12 positive experiences variables to predict overall helpfulness. Past positive experiences explained a significant amount ${ }^{70}$ of the variance in mental health professionals' overall helpfulness $\left(F(12,1196)=10.62, p<.001, R^{2}=\right.$ .10). As seen in Table 5, there were three unique predictors of mental health professionals' overall helpfulness: (1) focussing on topics participants had come to discuss, (2) affirming and validating identity, and (3) being knowledgeable about sex, sexuality, and gender diversity. These predictors seem to reflect key features of the therapeutic alliance proposed by Bordin (1979): agreement on goals for change, understanding of the tasks needed to reach the goals, and the development of bonds needed between client and therapist to complete this work. Specifically, affirming and validating identity is likely to strengthen bonds, focussing on topics participants had come to discuss demonstrates client-led goals, and gaining knowledge about sex, sexuality, and gender diversity might be one of the tasks needed to meet goals for change.

\footnotetext{
${ }^{70}$ While significant, the explained variance was relatively low. This is probably because I did not assess other important predictors of helpfulness such as the quality of the therapeutic alliance, participants' expectations about the helpfulness of therapy, and therapist skill and experience.
} 
Table 5

Multiple regression of overall helpfulness based on positive experiences

\begin{tabular}{lccc}
\hline & B & SE & $t$ \\
\hline Intercept & 2.87 & .06 & 50.50 \\
Asked how you identify & -.05 & .08 & -.66 \\
Asked about relationship between identity and & -.13 & .07 & -1.96 \\
mental health & & & \\
Asked about stigma and discrimination & -.04 & .07 & -.61 \\
experiences & .13 & .14 & .92 \\
Shared pronouns & .01 & .08 & .02 \\
Asked own understanding of identity & .09 & .10 & .91 \\
Checked pronouns & .32 & .06 & $5.08^{* *}$ \\
Focused on topics you'd come to discuss & -.03 & .07 & -.34 \\
Asked about coming out experiences & .07 & .07 & 1.10 \\
Displayed visual supports & -.05 & .07 & -.67 \\
Used inclusive language & .34 & .07 & $4.87 * *$ \\
Affirmed and validated & .23 & .07 & $3.40^{* *}$ \\
Knowledgeable & & & \\
\hline
\end{tabular}

Note. ${ }^{*} p \leq .05 ; * * p \leq .01$

To better understand the impact of past negative experiences, I conducted a multiple regression using the 13 negative experiences variables to predict overall helpfulness. Past negative experiences explained a significant amount ${ }^{71}$ of the variance in mental health professionals' overall helpfulness $\left(F(13,1195)=8.13, p<.001, R^{2}=\right.$ .08 ). As seen in Table 6 , there were four unique predictors of mental health professionals' overall helpfulness: (1) implying participants' identity was caused by past trauma, (2) making insensitive or hurtful comments about participants' identity, (3)

\footnotetext{
${ }^{71}$ As noted on the previous page the explained variance was significant but relatively low, probably because I only assessed some of the predictors of therapeutic helpfulness.
} 
making assumptions about identity, and (4) refusing to discuss identity when relevant. Returning to Bordin's (1979) features of the therapeutic alliance, the first three predictors are likely to interfere with the development of bonds between the therapist and their client, while the fourth predictor - refusing to discuss identity when relevant indicates that the therapist and client have not agreed on the goals of therapy. As such, these therapist behaviours might not be conducive to the development of a strong therapeutic alliance.

Table 6

Multiple regression of overall helpfulness based on negative experiences

\begin{tabular}{|c|c|c|c|}
\hline & B & $\mathrm{SE}$ & $t$ \\
\hline Intercept & 3.56 & .04 & 80.96 \\
\hline Refused to see you after you came out & -.16 & .25 & -.65 \\
\hline Used your deadname & .08 & .12 & .65 \\
\hline Implied your identity was a phase or not real & -.13 & .11 & -1.21 \\
\hline Tried to change your identity & .23 & .16 & 1.42 \\
\hline Implied your identity was caused by past trauma & -.22 & .09 & $-2.56^{*}$ \\
\hline Seemed surprised or uncomfortable when you came out & -.03 & .08 & -.33 \\
\hline Made insensitive or hurtful comments about identity & -.22 & .10 & $-2.27 *$ \\
\hline Assumed you were straight or cis & -.23 & .06 & $-3.72 * *$ \\
\hline $\begin{array}{l}\text { Refused to talk about your identity when you wanted to } \\
\text { discuss it }\end{array}$ & -.35 & .12 & $-2.86 * *$ \\
\hline Blamed your difficulties on your identity & -.14 & .09 & -1.60 \\
\hline Misgendered you & -.18 & .10 & -1.83 \\
\hline Focused on identity when it was not the issue & .01 & .08 & .02 \\
\hline Required education & .06 & .08 & .78 \\
\hline
\end{tabular}

Note. ${ }^{*} p \leq .05 ; * * p \leq .001$ 


\section{Discrimination Experiences and Future Worries}

To further explore the impact of other past experiences on future worries in mental health settings, I conducted a multiple regression analysis using the following items to predict combined worries: "I have heard stories of other queer, trans, and intersex folks having negative experiences with mental health professionals", "I have experienced discrimination outside of mental health settings on the basis of my sex, sexuality, and/or gender", and "I have had negative experiences with other health professionals in the past". Results indicated that the three predictors explained $15.1 \%$ of the variance in combined worries $\left(R^{2}=.15, F(3,1393)=82.67, p<.001\right)$. Hearing stories of other rainbow people having negative experiences with health professionals significantly predicted combined worries $(\beta=.20, p<.001)$, as did experiencing discrimination outside of mental health settings on the basis of rainbow identity $(\beta=$ $.19, p<.001)$, and having had negative experiences with other health professionals in the past $(\beta=.14, p<.001)$.

\section{Has the Experience Improved over Time?}

Next, I conducted a series of one-way ANOVAs to examine whether participants' experiences of accessing mental health support have improved over time. I first considered participant's combined positive experiences score. Participants who had seen a mental health professional within the last five years had a mean positive experiences score of $3.96(S D=2.89)$. Participants who had last seen a mental health professional more than five years ago had a mean positive experiences score of 2.30, $(S D=2.42)$. A one-way ANOVA showed there was a significant difference in positive experience scores between the two groups $(F(1,1285)=23.78, p<.001)$. Next, I considered participants' combined negative experiences score. Participants who had seen a mental health professional within the last five years had a mean negative experiences score of $2.40(S D=2.59)$. Participants who had last seen a mental health professional more than five years ago had a mean negative experiences score of 2.07 $(S D=2.35)$. A one-way ANOVA showed there was no significant difference in negative experience scores between the two groups $(F(1,1285)=1.19, p=.28)$.

Finally, I conducted a further one-way ANOVA to examine whether participants' scores on overall helpfulness of mental health professionals differed 
depending on when they had last seen a mental health professional (more than five years ago or within the last five years). There was no significant difference in overall helpfulness scores between the two groups $(F(1,14625)=1.85, p=.51)$. There was, however, a significant effect of recency of support on overall helpfulness when comparing participants who had last seen a mental health professional more than five years ago, within the last five years, within the last year, or who were currently seeing a mental health professional $(F(3,1460)=15.93, p<.001)$. Post hoc comparisons indicated that the mean helpfulness score for those currently accessing support $(M=$ $3.50, S D=.99)$ was significantly higher $(p<.05)$ than those who had accessed support more than five years ago $(M=3.06, S D=1.12)$, within the last five years $(M=3.10, S D$ $=1.03)$, and within the last year $(M=3.14, S D=1.03)$. There were no significant differences between those that had accessed support more than five years ago, within the last five years, or within the last year.

\section{Gender-affirming healthcare}

In order to explore whether the nature of mental health assessments for genderaffirming healthcare have changed over time, I conducted a series of chi-square tests examining the relationship between past experiences in mental health assessments (e.g. "the mental health professional asked when you first knew you were trans" and "the mental health professional asked whether you understood the physical effects of your healthcare") and the time participants had last undergone a mental health assessment for gender-affirming healthcare. Participants were either currently seeing a mental health professional $(n=21)$ or had seen a mental health professional more than ten years ago $(n=10)$, between five and ten years ago $(n=17)$, between one and five years ago $(n=$ $65)$, or within the last year $(n=21)$. There was no significant association between time of mental health assessment and any of the past experiences, $\chi^{2}$ 's $(4)=.26-8.57, p$ 's $>$ $.07 .^{72}$

\footnotetext{
${ }^{72}$ Chi-square tests are unreliable when at least $20 \%$ of cells have expected values less than five. Of the 15 chi-sqaure tests I ran, 15 had an expected value of less than five in more than $20 \%$ of cells. As such, I ran a second set of 15 chi-sqaure tests where I aggregated participants who had seen a mental health professional within the last year into one group, and more than one year ago into another group. None of these tests had an expected value of less than five in more than $20 \%$ of cells. Of these tests, all were nonsignificant $\left(\chi^{2}(1)=.01-3.27, p\right.$ 's $\left.>.07\right)$ except for the past experience "asked whether you understood the physical effects of your healthcare": a higher proportion than expected of participants who had accessed
} 
Because interview participants had described differences in gender-affirming healthcare provision based on location (see discussion of the "postcode lottery" on pages 129-136), I explored the relationship between location and reasons for not accessing gender-affirming healthcare among those who had not yet accessed care but wanted to access care. To do this, I conducted a series of chi-square tests examining reasons for not accessing care given by participants big cities, medium cities, small cities, towns, and rural areas. Dependent variables were whether or not participants reported that (1) they were unsure how to access gender-affirming care, (2) the healthcare they need is not available where they live, and (3) there is no point in accessing care, as the wait is too long. There was no significant relationship between location and participants reporting that they were unsure how to access genderaffirming care $\left(\chi^{2}(4)=7.25, p=.12\right)$ or that there is no point in accessing care, as the wait is too long $\left(\chi^{2}(4)=.55, p=.97\right)$. There was an association, however, between location and participants reporting that the healthcare they need is not available where they live $\left(\chi^{2}(4)=17.60, p=.001\right)$. Specifically, a higher proportion than expected of those living in rural areas reported that the healthcare they need is not available where they live, while a lower proportion than expected of those living in big cities reported that the healthcare they need is not available where they live.

\section{General Discussion}

Participants' responses to survey questions about access to mental health support provide further evidence that rainbow people in New Zealand have mixed experiences in therapy. Over half of participants found their mental health professionals mostly or extremely helpful overall, suggesting that many rainbow people in New Zealand have had positive experiences in mental health settings. The fact that nearly a third of participants found their mental health professionals overall mostly or extremely unhelpful, however, demonstrates that there is still considerable room for improvement when it comes to providing mental health support to rainbow people in Aotearoa.

care within the last year reported this experience than those who had accessed care more than one year ago, $\chi^{2}(1)=4.75, p=.03$. 
The finding that rainbow people have mixed experiences in mental health settings is consistent with international literature on rainbow therapy experiences (King et al., 2007; White \& Fontenot, 2019), and with previous New Zealand research (Adams et al., 2012; Birkenhead \& Rands, 2012; Semp, 2006; Welch et al., 2000). Participants' overall helpfulness ratings of mental health professionals (52.3\% as helpful, $28.2 \%$ as unhelpful, and $19.5 \%$ as neither helpful nor unhelpful) were similar to those reported in similar studies, such as Page's (2004) survey of bisexual women's experiences of mental health services in the US (where $49 \%$ of participants rated mental health professionals as helpful, 16\% as unhelpful, and 35\% as neither helpful nor unhelpful), and Bockting et al.'s (2004) study of trans patients' satisfaction with medical and psychiatric services (where trans patients' average satisfaction rating was 5.8 on a 7point scale, roughly halfway between 'neither satisfied nor dissatisfied' and 'very satisfied').

It is interesting to note that interview participants tended to focus on the negative aspects of their experiences accessing mental health support, whereas survey findings indicate that, overall, mental health professionals are more often helpful than not. It may be that interview participants had more negative experiences on average than survey participants, however I think this discrepancy is more likely attributable to that fact that my interview questions were more heavily focussed on what could be improved in mental health settings than on what is going well. There is also evidence to suggest that negative experiences generally have more impact than positive experiences (e.g. Baumeister, Bratslavsky, Finkenauer, \& Vohs, 2001), which may also explain this discrepancy between interview and survey findings.

Aside from the discrepancies in overall helpfulness between the survey and interview findings, findings from the online survey were broadly consistent with the interview findings reported in Chapters 6 and 7. In both studies there was variation in the extent to which participants felt comfortable coming out in mental health settings, and variation in levels of comfort discussing sexuality and gender. Participants reported less comfort telling professionals about their sexuality than reported in McCann and Sharek's (2014) survey of LGBT people's experiences of mental health services in Ireland; in this study, $31 \%$ of participants felt they could not discuss their LGBT identity with to their mental health provider, while $49 \%$ felt they could. In the current 
study, $43.3 \%$ of participants reported feeling uncomfortable about telling a new mental health professional about their sexuality, while $44 \%$ reported feeling comfortable. It is worth noting, however, that the extent to which someone feels comfortable disclosing their identity and able to discuss their identity may not be directly comparable.

It was encouraging to note that nearly half of participants who had accessed support in the last five years found their mental health professionals knowledgeable, however this means that more than half of these participants had not encountered a mental health professionals who they found knowledgeable about sexuality, gender, and sex characteristic diversity. This finding is consistent with results from numerous past studies showing that a lack of knowledge is one of the most common negative experiences for rainbow people in mental health settings (Hunt et al., 2006; Lucksted, 2004; McCullough et al., 2017; Poteat et al., 2013; Shelton \& Delgado-Romero, 2011). Responses to questions about past experiences in mental health settings also highlighted that it was common for participants to report mental health professionals had made assumptions about their gender or sexuality or had seemed surprised or uncomfortable when the client came out, which has also been widely reported in previous research (Eady et al., 2011; Evans \& Barker, 2010; Hunt et al., 2006; Liddle, 1996; McCullough et al., 2017; Robertson, 1998; Saulnier \& Wheeler, 2000). My research extended on these previous studies by exploring the helpfulness of a number of safety signals: actions that can signal a professional is rainbow-friendly. Results suggested that using inclusive language and displaying visual signs of support (such as rainbow flyers and posters) are most helpful for creating a rainbow-friendly space.

More recent research studies (e.g. Eady et al., 2011; McCann \& Sharek, 2014; McCullough et al., 2017; Shelton \& Delgado-Romero, 2011) tend to report fewer instances of overt discrimination than older research studies (e.g. Golding, 1997; Lucksted, 2004; McFarlane, 1998; Nystrom, 1997), however my survey results suggest that there has been little change in participants' negative experiences over time. An alternative explanation is that my measure of change over time missed some of the nuances in the nature of past experiences, and how they are interpreted by participants. I assessed change in the number of negative experiences, rather than the type of past experiences. Not all experiences are likely to be given equal weight; for example, a mental health professional attempting to change a participants' identity will likely be 
experienced as more negative than a surprised look from their mental health professional when they came out.

I was surprised to find that only a few past experiences significantly predicted participants' overall helpfulness ratings in the two multiple regression analyses. It is possible that some past experiences did not significantly predict overall helpfulness ratings because they had low base rates. For example, only $1.5 \%$ of participants who had accessed support in the last five years had a mental health professional refuse to see them anymore after they came out. The few unique predictors of overall helpfulness could also be explained by the fact that some past experiences significantly predict overall helpfulness when entered into a simple linear regression analysis, ${ }^{73}$ but were not significant predictors when entered into a multiple regression. This suggests that other past experiences accounted for their contribution to the overall helpfulness rating.

Responses to questions about gender-affirming healthcare highlighted significant barriers to access, and were consistent with interview findings and wider literature about widespread gatekeeping and a need to tell a particular trans narrative in order to access care (Ellis et al., 2015; McNeil et al., 2012; Violeta \& Langer, 2017). The findings emphasise the importance of ensuring consistency of care in different regions across Aotearoa, and of making clear the role of the mental health professional in the provision of gender-affirming care. The fact that the proportion of participants reporting various past experiences in relation to gender-affirming healthcare had not changed over time could suggest that this is an area where progress is slow, but could also reflect the small number of participants who had accessed gender-affirming healthcare more than five years ago. With such small sample sizes, is is possible I did not have the statistical power necessary to detect change over time in gender-affirming healthcare assessments.

\footnotetext{
${ }^{73}$ For example, the item "the mental health professional implied your identity was a phase or not real" significantly predicted overall helpfulness when entered into a simple linear regression on its own $\left(F(1,1207)=30.00, p<.001, R^{2}=.02\right)$, as did the item "the mental health professional seemed surprised or uncomfortable when you came out" $\left(F(1,1207)=20.31, p<.001, R^{2}=.02\right)$.
} 


\section{Implications}

The findings that previous experiences in mental health settings are related to future worries and feelings of comfort are hardly surprising; that our past experiences influence our future expectations is one of the most basic principles of psychological learning theories (Tennyson \& Volk, 2015). Nonetheless, this is an important point for mental health professionals to keep in mind - that clients bring all their previous experiences into therapy settings. Mental health professionals must, therefore, earn the trust of their clients, rather than expect they will have it by virtue of their position. Survey results emphasise that there are many small actions that mental health professionals can take to communicate that they are rainbow-friendly (e.g. sharing and checking pronouns, using inclusive language, and displaying visual signs of support), and highlight the importance of affirmation, validation, knowledge about sexuality, gender, and sex characteristic diversity, and focussing on the topics clients have come to discuss. Survey results also highlight how damaging it can be when mental health professionals make assumptions or insensitive comments about rainbow identities, refuse to discuss identity when it is relevant, or link rainbow identities with past trauma.

Results showing demographic differences on select survey items calls attention to the heterogenous nature of rainbow people and highlights the importance of intersectionality. Although all participants had a rainbow identity, they also had other important demographic characteristics (e.g. ethnicity, age, region) that impacted on their experiences in mental health settings in different ways. While there is a lack of research about the experiences of minority groups within the rainbow community who access mental health support, (for exceptions, see McCann, Lee, \& Brown, 2016; Reading \& Rubin, 2011; Wynn \& West-Olatunji, 2009), the demographic differences seen in this study are consistent with those showing that various minority groups have elevated rates of mental health difficulties (Chiang et al., 2017; Hopkinson et al., 2017; Yarns, Abrams, Meeks, \& Sewell, 2016) and are likely to encounter barriers to accessing healthcare (O’Toole \& Brown, 2003; Solway, Estes, Goldberg, \& Berry, 2010). It was surprising to see few differences in the current study across ethnic groups, and no differences based on location, whether participants were migrants, and whether participants were religious. This could be due to low sample sizes of double minority groups and, for migrants, could be related to the heterogenity of this group; migrants 
coming from countries that are culturally similar to New Zealand may not experience the same difficulties as those coming from countries that are culturally dissimilar.

Like the interview study and past literature, survey results highlight the need for mental health professionals to seek education and training about sexuality, gender, and sex characteristic diversity, so that their clients are not left to do this work for them (Bowers et al., 2005; Eady et al., 2011; Evans \& Barker, 2010; Lucksted, 2004). As noted in the previous chapter, the findings of my interview and survey studies provide valuable information to address the knowledge gaps of mental health professionals. In the next chapter, I describe process of developing a resource for mental health professionals that helps to guide their work with rainbow clients.

\section{Strengths of the Study}

The large size of the online survey sample is a key strength of this study. This is the largest ever study of rainbow mental health support experiences in New Zealand, and is the second-largest study of any kind with rainbow people in New Zealand (the largest being the Lavender Islands study; Henrickson, Neville, Jordan, \& Donaghey, 2007). With a sample size of 1575 I was able to conduct analyses of group differences that were not possible in previous studies about rainbow experiences of accessing mental health support. The number and range of survey measures is another strength of this study, as this allowed me to explore relationships between past experiences and future expectations and to investigate change over time. Such analyses have been almost entirely absent from previous quantitative work in this area. The inclusion of measures about gender-affirming healthcare provides insight into trans peoples' experiences of mental health assessments for the first time in New Zealand, which is essential for understanding how to improve this process for trans and nonbinary New Zealanders.

The community collaboration in survey design and recruitment is another strength of this study. By seeking guidance from community organisations and advocates I ensured that questions were worded respectfully, and added in measures that I had originally not thought to include. This provided rich data that not only answer my research questions, but that community organisations will be able to use in their own work. Without the community partnerships and collaborations I built prior to this study, I doubt we would have been able to reach so many rainbow people with our survey. 


\section{Limitations of the Study}

In spite of the large sample, a key limitation of the study was a lack of success in recruiting minority groups within the rainbow community, such as ethnic minorities (other than Māori), older people, people living rurally or in smaller towns and cities, refugees, and intersex people. When we did recruit minorities within the rainbow community (such as neurodiverse people and people with physical disabilities) there were no questions specifically about experiences and needs related to their intersecting identity. This limits my ability to provide guidance about supporting people in these groups in my future resource, necessitating further research to inform these parts of the resource. Additionally, although this study was not designed to be representative of all rainbow people, its large sample size may mean that some take it to be reflective of rainbow peoples' experiences of acessing mental health support in Aotearoa. While I think it certainly provides helpful insight into this experience, the fact that findings are primarily based on the experiences of majority groups within rainbow communities means it should not be used to make claims about the experiences of New Zealand's rainbow people in general.

Other limitations of the study relate to survey design. Some items seem to have been interpreted in different ways by different participants, or were potentially worded in an ambigious manner. For example, the past experience "the mental health professional asked about the relationship between my identity and my mental health" was originally intended for inclusion as a 'positive' experience, as interview participants reported that they would appreciate their mental health professional exploring their understanding of this relationship. When this item was uncorrelated with overall helpfulness and positively correlated with future worries of discrimination and discomfort from a mental health professional, I wondered whether it had been interpreted as mental health professionals implying a causal connection between participants' identities and mental health (i.e. taking a pathologising view). If I were to redesign the survey I might change the wording of this item to be "the mental health professional asked about your own understanding of the relationship between your identity and mental health."

Another limitation related to survey design is my choice not to include a "does not apply" option in questions about past experiences in mental health settings. In cases 
where participants left all these items blank, it was unclear whether they had done so because they had not experienced any of the listed experiences, or because they chose to skip that question. My original reasoning for not including a "does not apply" option for these questions was that it would have made an already lengthy survey even longer. When analysing the data, however, I realised that I had to be careful about the claims I made in relation to this data. I eventually decided that if participants had left all these items blank I would code their response to this set of questions as missing (as not having experienced any of the 25 listed experiences seemed unlikely), however not having experienced any of the listed experiences is not outside the realm of possibility, so I may have incorrectly attributed some of these responses.

\section{Future Directions}

While the analyses above address the specific research questions identified on page 3 , there is clearly opportunity to investigate the data further. In future, I am particularly interested in examining data from the open-ended survey measures, as time and space constraints prevented me from analysing this qualitative data as part of this thesis. I am also eager to explore demographic variables as potential moderating factors: for example, does the strength of the relationship between past discrimination experiences and future worries in mental health settings change depending on participants' age, sexual orientation, or gender? Other potential further analyses include latent profile analyses, which would allow me to examine different profiles of rainbow clients based on their expectations, worries and concerns, and what they would find helpful in mental health settings. Understanding different profiles of participants could have implications for how mental health professionals may modify their practice to meet the needs of different clients.

Future research could build on the current study by asking participants to evaluate their past therapy experiences (e.g. "the mental health professional affirmed and validated your identity") as positive or negative, or helpful or unhelpful (rather than this being determined by the researcher), and to reflect on the relative impact of these experiences. This could help to identify the most important factors for resources and training. For example, it may be more important to focus on affirmation and validation in rainbow cultural competence training, and less important to focus on sharing 
pronouns. Future research should also address limitations of the current study by making an effort to recruit groups within the rainbow community who were underrepresented in the current study, including ethnic minorities, older people, people living outside of larger cities, refugees, and intersex people.

Finally, the fact that nearly a third of participants found mental health professionals mostly or extremely unhelpful overall raises questions about what it must be like to seek out support following a negative experience. Presumably negative experiences would reduce hope for finding effective support, and continuing to seek out support would require a great deal of resilience. While I have briefly considered this in my interview and survey studies, future research would benefit from a more in-depth exploration of rainbow peoples' help-seeking following negative mental health support experiences.

\section{Conclusion}

This online survey explored the experiences of 1575 rainbow people who had accessed mental health support, and 254 trans and nonbinary people who had accessed, or were in the process of accessing, gender-affirming healthcare. Survey findings showed mixed experiences in therapeutic settings. Participants varied in the extent to which they worried about discrimination when meeting a new mental health professional, or felt uncomfortable coming out and discussing sexuality and gender. There tended to be, however, a relationship between participants' past experiences in mental health settings and expectations for future experiences in mental health settings. Findings related to gender-affirming healthcare showed widespread structural barriers to accessing care, and many participants reported a need to tell a particular narrative in order to access care. Like my interview analysis, these findings highlight the importance of increasing resources in New Zealand's public mental health services in order to facilitate healthcare access, and emphasises a need for mental health professionals to increase their rainbow cultural competence.

In the next chapter I present the resource I developed based on these survey findings and the interview findings presented in Chapters 6 and 7. This resource is not sufficient to address the wider problems around rainbow cultural competence in New Zealand's mental health sector; this would require significant change in our mental 
health training programmes, and may also require change in professional associations' requirements for ongoing professional development or professional competence training. Despite this, presenting participants' experiences, together with with some background knowledge and therapeutic guidance, is a step towards filling the knowledge gap around rainbow needs and experiences among New Zealand's mental health professionals. 


\section{Chapter 9: The Resource}

The overarching goal of this research project was to understand the experiences of rainbow people who had accessed mental health support and gender-affirming healthcare in Aotearoa, in order to explore how mental health service provision can be improved for queer, trans, and intersex people. Findings from interviews and online surveys with almost 1600 rainbow people across Aotearoa showed that they have had mixed experiences in New Zealand's mental health services, and that mental health professionals often lacked knowledge about sexuality, gender, and sex characteristic diversity. Mental health professionals also often lacked expertise in how to incorporate conversations about rainbow identities into therapy. These findings demonstrate a clear need for increased rainbow cultural competence among New Zealand's mental health professionals.

To meet this need for increased rainbow cultural competence, the final stage in my research process was the development of an evidence-based resource to guide mental health professionals in their work with rainbow clients. The resource synthesised information from my research findings, existing literature, and feedback from mental health professionals and rainbow community members. This chapter documents the processes of resource development, including seeking and incorporating feedback into the resource. It then concludes with the resource that was printed and distributed to services and organisations across the country. The resource is freely available online for download at https://rainbowmentalhealth.nz.

\section{Resource Development}

The first decision to make about resource development was what form the resource should take. Like all research-related decisions, I discussed this with my supervisors, research collaborators, and members of the wider rainbow and mental health communities. I settled quickly on a written resource, with a plan to consider a video resource later (if time and funding permitted). I wanted to strike a balance between a dense, text heavy resource (e.g. the British Psychological Society's 2012 
Guidelines for Psychologists, which are informative but lengthy, totalling almost 50,000 words; Shaw et al., 2012), and a resource that was too general to be of practical use for mental health professionals. An A5 booklet, similar to Elizabeth Kerekere's takatāpui resources (Kerekere, 2015, 2017b), seemed the most efficient and accessible way to provide information to mental health professionals.

Many rainbow resources include photos or illustrations of rainbow community members (e.g. InsideOUT, 2016, 2017; Orchard, 2017). In some contexts this representation is important, as it allows rainbow people (particularly rainbow young people) to see themselves in publications and media in a way that they may not have before. For this resource, however, I wanted to avoid visually representing rainbow people, as was concerned this could reinforce misconceptions that rainbow people can be differentiated by their appearance (e.g. by the way they wear their hair, their clothes, or by piercings and tattoos). Instead, I wanted to communicate to mental health professionals that anyone who walked through their door could be part of the rainbow community. Because of this, I came up with the idea of a native birds and plants theme for the resource, to ensure the resource was Aotearoa-centred. I asked my research partners how they felt about participants' quotes coming from different kinds of native birds, like tui, kākāa, and tauhou. All were supportive, so we set about finding an artist for the resource. After careful consideration I approached Bo Moore, a Wellingtonbased artist who had donated a considerable amount of time to InsideOUT. Bo had experience drawing New Zealand's plants and animals, and was able to design the resource, as well as illustrate it.

To develop the initial resource draft, I began by brainstorming topics that were important to include based on my interview and online survey findings (e.g. terminology, minority stress, creating a rainbow-friendly space, and gender-affirming healthcare.). I considered how these topics might best be ordered into broader sections of the resource. Once I had a list of topics I drafted each section, incorporating quotes from interview participants that reflected and supported my points. I sent this first draft to my community collaborators, as well as to a handful of other mental health professionals and rainbow community members. I also sought guidance on specific sections of the resource from relevant experts (e.g. a representative from Intersex Awareness New Zealand for the Intersex/Variations in Sex Characteristics section). At 
this point I held a hui at RainbowYOUTH to bring together the Auckland-based advisors on the project and met with InsideOUT and Gender Minorities Aotearoa to discuss the initial draft of the resource with Wellington-based advisors.

This initial consultation led to some key changes in the resource draft. We removed the 'privilege checklist' that was originally included in the resource (as someone pointed out that such checklists can invisibilise the marginalised within a dominant group), created a section on the 'Historical Context' page to specifically discuss trans and intersex histories, and removed reference to 'passing' in the 'GenderAffirming Healthcare' section. The hui at RainbowYOUTH and InsideOUT saw spirited discussions about the sections then titled "Men in the Rainbow Community" and "Women in the Rainbow Community", which attempted to outline some of the ways in which rainbow people are affected by gender norms and stereotypes. Although I had assumed it was obvious that trans men and women were included in this section, this was not clear to all readers. Moreover, there was a concern that this section reified a binary understanding of gender. As such, this section was later revised and renamed "Gender Norms and Rainbow Identities."

\section{Gathering Feedback}

Once I had incorporated all initial feedback into the resource draft, I sought feedback from a wider group of stakeholders (mental health professionals and rainbow community members). I collected feedback using two different methods - confidential feedback, where I invited people I already had a working relationship with to send me their feedback via email, and anonymous feedback, where I invited all others (including past participants who had expressed their interest in giving resource feedback) to send feedback via a short Qualtrics survey. ${ }^{74}$ The anonymous feedback survey had two versions: one for mental health professionals and one for rainbow community members.

\footnotetext{
${ }^{74}$ I chose these two methods because I did not want to ask people who I knew well, or who had already given a great deal of time and energy to the project, to take a role similar to that of a research participant. On the other hand, I wanted to give people the opportunity to give their honest feedback without being identified. In some cases, I gave people the option of either emailing their feedback directly to me or clicking the Qualtrics link and providing it there, depending on their preference.
} 
Data collection for this part of the project was approved by the Victoria University of Wellington Human Ethics Committee (RM\# 0000027274; see Appendix L).

Mental health professionals were recruited using a flyer distributed online (see Figure 18). This flyer adopted the same design and layout of the recruitment flyers used for the interviews and online survey, revised to target mental health professionals, rather than rainbow community members. Potential participants were invited to take part if they had worked or were currently working or training as a mental health professional in New Zealand (a psychologist, counsellor, psychiatrist, therapist, social worker, mental health nurse, etc.), and were interested in giving feedback about an upcoming resource about supporting rainbow clients in mental health settings. The poster also noted that knowledge and experience working with rainbow community members was not required, as we wanted to hear a wide range of different perspectives.

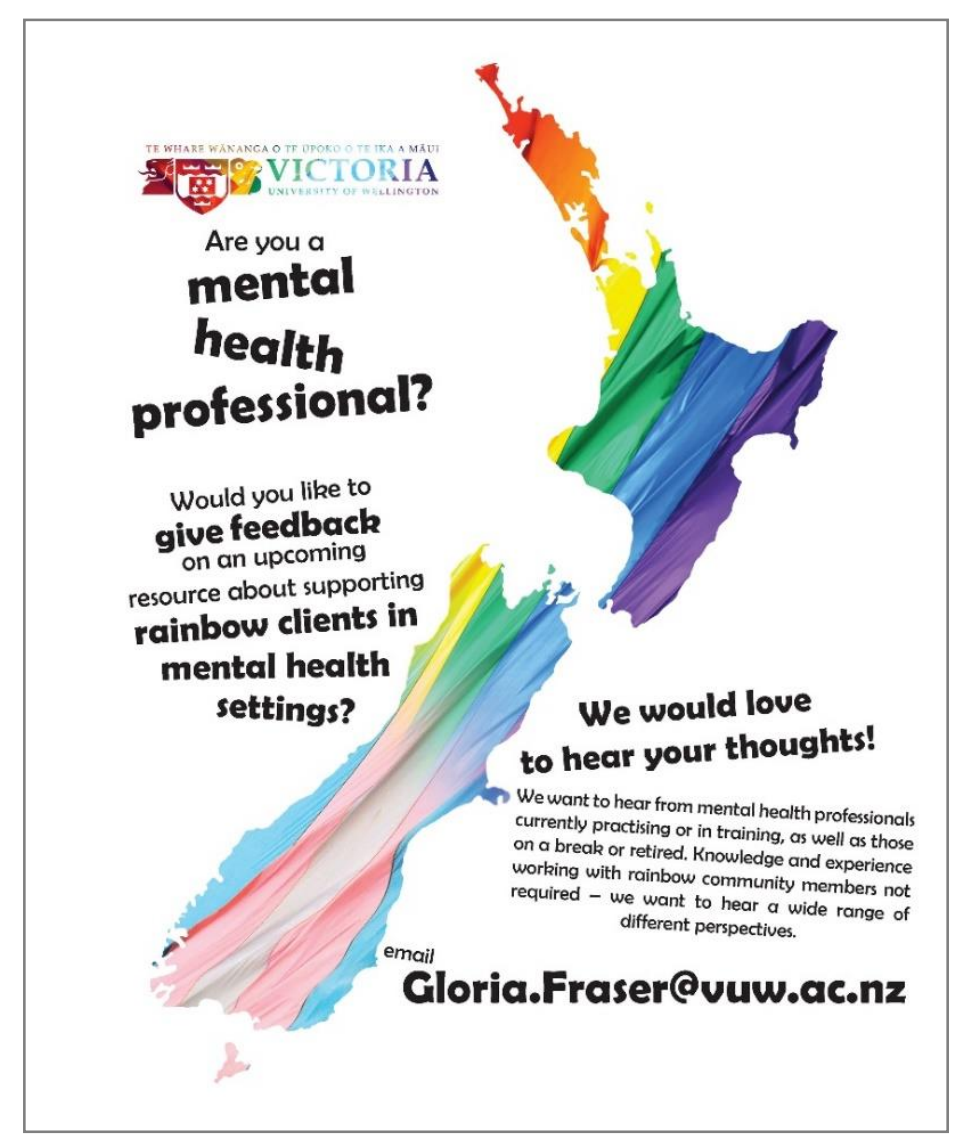

Figure 18. Resource feedback recruitment flyer for mental health professionals. 
I posted this flyer to my personal Facebook page, to the Facebook page I had created to recruit for the online survey (https://facebook.com/rainbowmentalhealth/), and to various Facebook groups, including a group for Victoria University of Wellington clinical psychology students and a group for Māori postgraduate students in psychology. I also sent the flyer to all staff of my clinical psychology programme, to all those on the Youth Wellbeing Study mailing list (the research team I am part of in the School of Psychology), to mental health professionals and organisations that had supported our online survey recruitment, and to professional organisations including the New Zealand College of Clinical Psychologists, the New Zealand Psychological Society, the Royal Australian and New Zealand College of Psychiatrists, the New Zealand Association of Psychotherapists, and the New Zealand Association of Counsellors.

In total, 96 people contacted me to express interest in providing resource feedback. In response, I sent them an email explaining what resource feedback would entail (see Appendix M). The email included a link to the resource draft and a link to the Qualtrics feedback survey. If the person did not wish to give feedback but wanted to see the resource when it was finalised, they also had the option of clicking a third link to a Qualtrics survey where they could provide their contact details in order to be notified when the final resource was available. Upon clicking the Qualtrics survey link, potential participants were given additional information about the project and study (see Appendix N). Those that consented to providing resource feedback were asked to confirm whether they were currently working or training as a mental health professional, or whether they had worked as a mental health professional in the past. They were then asked the following set of questions: 


\begin{tabular}{|c|c|c|c|c|c|}
\hline \multicolumn{6}{|c|}{ Please rate the extent to which you agree or disagree with the following statements: } \\
\hline & $\begin{array}{l}\text { Strongly } \\
\text { disagree }\end{array}$ & $\begin{array}{c}\text { Slightly } \\
\text { disagree }\end{array}$ & $\begin{array}{l}\text { Neither } \\
\text { agree } \\
\text { nor } \\
\text { disagree }\end{array}$ & $\begin{array}{l}\text { Slightly } \\
\text { agree }\end{array}$ & $\begin{array}{c}\text { Strongly } \\
\text { agree }\end{array}$ \\
\hline I found the resource easy to understand & $\mathrm{O}$ & $\mathrm{O}$ & $\mathrm{O}$ & O & $\mathrm{O}$ \\
\hline $\begin{array}{l}\text { I think other mental health professionals will } \\
\text { understand the information in the resource }\end{array}$ & $\mathrm{O}$ & $\mathrm{O}$ & $\mathrm{O}$ & 0 & 0 \\
\hline $\begin{array}{l}\text { In my opinion, the information provided in the } \\
\text { resource is accurate }\end{array}$ & $\mathrm{O}$ & 0 & $\mathrm{O}$ & 0 & $\mathrm{O}$ \\
\hline $\begin{array}{l}\text { I think this resource will help mental health } \\
\text { professionals to support queer, trans, and } \\
\text { intersex people }\end{array}$ & $\mathrm{O}$ & $\mathrm{O}$ & $\mathrm{O}$ & O & $\mathrm{O}$ \\
\hline I would use this resource in my own practice & $\mathrm{O}$ & $\mathrm{O}$ & $\mathrm{O}$ & $\mathrm{O}$ & $\mathrm{O}$ \\
\hline I found the language in the resource respectful & $\mathrm{O}$ & 0 & 0 & 0 & 0 \\
\hline $\begin{array}{l}\text { This resource answered questions I had about } \\
\text { working with rainbow community members }\end{array}$ & $\mathrm{O}$ & $\mathrm{O}$ & $\mathrm{O}$ & ○ & $\mathrm{O}$ \\
\hline $\begin{array}{l}\text { I think the resource captured the diversity } \\
\text { within the rainbow community }\end{array}$ & $\mathrm{O}$ & $\mathrm{O}$ & $\mathrm{O}$ & O & $\mathrm{O}$ \\
\hline
\end{tabular}

Figure 19. Resource feedback questions for mental health professionals.

Participants were also asked to rate how much experience they had working with rainbow people (on a 5-point Likert scale where $1=$ A great deal and $5=$ None at all) and how confident they felt about their ability to support rainbow clients in mental health settings (on a 5-point Likert scale where $1=$ Extremely confident and $5=$ Not at all confident). Finally, they were invited to share any other feedback about the resource in an open-text box.

Rainbow participants were recruited for resource feedback directly. I sent an email (see Appendix O) to all online survey participants who had indicated at the end of the survey that they would like to be involved in the resource development process $(n=$ 406). The email contained a link to the Qualtrics feedback survey, a link to the resource draft, and a link to a second Qualtrics survey where they could provide their contact details in order to be notified when the final resource was available. Upon clicking the link to the Qualtrics survey, potential participants were given additional information 
about the project and study (see Appendix P). Those that consented to providing resource feedback were then asked the following set of questions:

Please rate the extent to which you agree or disagree with the following statements:

I found the resource easy to understand
$\begin{aligned} & \text { I think mental health professionals will } \\ & \text { understand the information in the resource }\end{aligned}$
$\begin{aligned} & \text { In my opinion, the information provided in the } \\ & \text { resource is accurate }\end{aligned}$
$\begin{aligned} & \text { I would recommend the resource to other } \\ & \text { people }\end{aligned}$
$\begin{aligned} & \text { I think this resource will help mental health } \\ & \text { professionals to support queer, trans, and } \\ & \text { intersex people }\end{aligned}$
$\begin{aligned} & \text { I found the language in the resource respectful } \\ & \text { disagree }\end{aligned}$

Figure 20. Resource feedback questions for rainbow participants.

Finally, rainbow participants were invited to share any other feedback about the resource in an open-text box.

In total, we received feedback from 108 people. Table 7 presents a breakdown of resource feedback. ${ }^{75-76}$

75 This table does not include responses from my PhD supervisors or community partners (InsideOUT, RainbowYOUTH, and Gender Minorities Aotearoa). Anonymous feedback responses were included if participants responded to at least one of the items pertaining to the content of the resource.

${ }^{76}$ Many people who gave feedback were both rainbow community members and mental health professionals; those in this group that gave anonymous feedback were categorised according to which survey they completed, and those in this group that gave identifiable feedback were placed into one or other category depending on the capacity in which they were primarily invited to take part. In retrospect, it would have been helpful to include questions that allowed us to determine how many participants fell into both categories, however we chose not to include demographic information in our Qualtrics surveys in order to keep them brief. 
Table 7

Breakdown of resource feedback

\begin{tabular}{lccc}
\hline & $\begin{array}{c}\text { Confidential } \\
\text { feedback }\end{array}$ & $\begin{array}{c}\text { Anonymous } \\
\text { feedback }\end{array}$ & Total \\
\hline Rainbow community members & 12 & 40 & 52 \\
Mental health professionals & 14 & 42 & 56 \\
Total & 26 & 82 & 108 \\
\hline
\end{tabular}

\section{Quantitative Feedback}

Of the mental health professionals who gave feedback using the anonymous Qualtrics survey, 64.6\% $(n=31)$ were working as a mental health professional, 25\% were training to be a mental health professional, and $10.4 \%$ had worked as a mental health professional in the past. There were a range of responses regarding mental health professionals' self-reported experience working with rainbow clients in mental health settings $(M=3.07, S D=1.28)$ and self-reported confidence working with rainbow clients in mental health settings $(M=2.55, S D=.83)$. This was encouraging, as I had been concerned that we might only receive feedback from professionals who had done a lot of work with rainbow clients; I was eager to hear how professionals with less confidence and experience found the resource, as it is these professionals who may benefit most from reading it.

Table 8 shows mental health professionals' and rainbow participants' quantitative responses to items regarding the content of the resource ( 1 = Strongly disagree and 5 = Strongly agree). Mental health professionals' mean responses to all items fell between "Agree" and "Strongly agree", suggesting they found the resource informative, clear, and helpful. Bivariate correlations showed no relationship between any of the resource evaluation items and professionals' self-reported confidence and experience, though there was a strong positive correlation between professionals' level of self-reported experience and self-reported confidence $(r=.67, p<.001)$. Rainbow community members' mean responses to all items fell between "Agree" and "Strongly agree", suggesting that they too found the resource informative, clear, and helpful. 
Table 8

Resource feedback from mental health professionals and rainbow participants

\begin{tabular}{|c|c|c|}
\hline & $\begin{array}{l}\text { Mental health } \\
\text { professionals } \\
M(S D)\end{array}$ & $\begin{array}{l}\text { Rainbow } \\
\text { participants } \\
\quad M(S D)\end{array}$ \\
\hline I found the resource easy to understand & $4.69(.92)$ & $4.60(.87)$ \\
\hline $\begin{array}{l}\text { I think mental health professionals will understand } \\
\text { the information in this resource }\end{array}$ & $4.52(1.02)$ & $4.43(.81)$ \\
\hline $\begin{array}{l}\text { In my opinion, the information provided in this } \\
\text { resource is accurate }\end{array}$ & $4.57(.77)$ & $4.38(.98)$ \\
\hline $\begin{array}{l}\text { I think this resource will help mental health } \\
\text { professionals to support queer, trans, and intersex } \\
\text { people }\end{array}$ & $4.60(.83)$ & $4.55(.75)$ \\
\hline I found the language in this resource respectful & $4.81(.67)$ & $4.65(.83)$ \\
\hline $\begin{array}{l}\text { I think this resource captured the diversity within the } \\
\text { rainbow community }\end{array}$ & $4.71(.77)$ & $4.40(.90)$ \\
\hline I would use this resource in my own practice & $4.67(.75)$ & - \\
\hline $\begin{array}{l}\text { This resource answered questions I had about } \\
\text { working with rainbow community members }\end{array}$ & $4.14(1.14)$ & - \\
\hline I would recommend the resource to other people & - & $4.50(.99)$ \\
\hline I felt represented by this resource & - & $4.25(1.08)$ \\
\hline
\end{tabular}

\section{Qualitative Feedback}

While quantitative feedback from mental health professionals and rainbow community members showed that the resource was well-received, it did not shed light on how the resource could be improved. Because of this, we also collected qualitative data about the resource, to consider potential changes to the resource draft. The qualitative feedback from mental health professionals and rainbow community members closely reflected the quantitative feedback, with a number of participants commending the resource:

Mental health professional: Wonderful to have new material that is relevant, up-to-date and wide ranging. I liked the straight forward language and how 
comprehensive the resource is. Grateful to have people in our community engaged in the 'normalising' process for the queer and trans communities. I always hope that the more we talk and are visible, that hopefully for youth their environment will be more accepting and create fewer mental health difficulties. Thank you and ngā mihi mahana Gloria.

Rainbow community member: Great stuff! :) Looking forward to seeing the completed version. I feel fully represented, and I hope this resource is promoted/distributed and readily available online to all of our medical professionals.

Feedback about how the resource could be improved most commonly focussed on wording and terminology use, suggestions for where some sections could be extended (e.g. providing gender-affirming healthcare to children and young people), and requests for additional sections to be included (e.g. supporting rainbow people with disabilities).

\section{Incorporating Feedback}

A number of changes were made in response to the feedback. For example, I changed "communities" to "people" throughout, to reflect that many rainbow people either do not have the opportunity to be a part of rainbow communities (e.g. if they live rurally), or do not feel part of rainbow communities. I also removed terms that I did not have the space to define or explain (e.g. erasure) and clarified definitions of other terms (e.g. rainbow). I added sections about supporting rainbow people with disabilities, and rainbow refugees and asylum seekers. Finally, I revised the section about genderaffirming healthcare to better reflect the current context of gender-affirming healthcare provision in Aotearoa. When incorporating feedback, I considered whether the feedback was 'one-off' or had been suggested by several participants, and whether it reflected our research findings and kaupapa. I also considered the space it would take to incorporate the feedback. In some cases, I wanted to incorporate feedback (e.g. adding a section about the experiences of older adults) but decided that doing so would make the resource too long. ${ }^{77}$

\footnotetext{
${ }^{77}$ I aimed to keep the resource at or below 10,000 words. This was in part because I thought mental health professionals were less likely to read the resource if it was too lengthy, and also because we had a
} 


\section{Challenges of Incorporating Feedback}

The process of revising the resource was undoubtedly the most challenging part of my entire research project. Perhaps unsurprisingly, it was not unusual to receive opposing feedback on some resource sections (e.g. terminology and the wording of suggested questions) from so large a group of people. In cases where I decided to add new sections to the resource (e.g. supporting rainbow people with disabilities and rainbow refugees and migrants), I limited myself to around 250 words. Upon seeking advice from disability and refugee advocates and community members, however, I was informed by some that it would be impossible to do such complex topics justice in so few words (and, indeed, that it could be dangerous for therapists to think they are culturally equipped and competent just after reading one page from a resource). This left me wondering whether it was better to leave these topics out of the resource altogether. I eventually decided to include as much information as possible on a single page, then link to further resources and reading at the back of the booklet. Although imperfect, I thought it better to include some, albeit limited, information than to contribute to the erasure of already invisibilised groups by putting them in the 'too hard' basket.

Much of the resource feedback closely reflected discussions happening in the wider rainbow community at the time of resource development. For example, we received feedback from some gay and lesbian people who felt there was not enough content specifically related to their needs and experiences, with one person requesting resource sections dedicated to gay and lesbian people (as we had done for other groups), and another suggesting that we had focussed on multiple gender attracted people and trans people at the expense of gay and lesbian people. I found this feedback challenging because some of the comments felt uncomfortably close to trans-exclusionary sentiments (see Compton, 2019 for discussion), but at the same time I felt it would be inappropriate for me to weigh in as a straight cis woman.

My usual strategy of consulting with community partners was complicated in this instance by the fact that my community advisors brought their own subject

limited budget for printing and translating the resource; if our resource ran much longer than 10,000 words we either would not have been able to translate the entire document into te reo Māori, or we would have printed significantly fewer hard copies for distribution. 
positions to the conversation, and in some cases had little time for gay and lesbian community members who felt they were being erased in the resource. I then had a frank (and very helpful) conversation with one community advisor (a lesbian herself), who pointed out that there is a difference between being seen and being centred: the needs of gay (and, to a lesser extent, lesbian) people have historically been prioritised over those of other sexual minorities, as well as those of trans and intersex people, and our explicit focus on these groups in this resource was an attempt to highlight the needs and experiences of those who have previously been left out of the conversation. In saying this, we searched the resource together and realised that the word "lesbian" was only included once (ironically, on the page about supporting multiple gender attracted people). To address this, we decided to specifically mention lesbians when discussing the formation of Gay Liberation groups, and on the page about gender norms and rainbow identities, as many stereotypes about queer women are specifically targeted at lesbians.

\section{Sharing the Resource}

\section{Resource Translation and Accessibility}

When developing the resource, I felt strongly that it should exist both in te reo Pākehā and te reo Māori. ${ }^{78}$ This is not only because te reo Māori is an official language of Aotearoa, but because I saw this as an opportunity to promote Māori language use and revitalisation. As with many of the bright ideas I had over the course of this project, translating the resource into Māori turned out to be much more complicated than I initially thought it would. First, it was difficult to find a translator with the knowledge and time, and who we could afford to pay from our research budget. We were very lucky to have Hēmi Kelly (lecturer in te reo Māori and a licensed translator) complete the translation, with the support of a team of experienced translators. Hēmi shared that he was eager to assist, being takatāpui himself.

A second challenge in the process of translating the resource was the fact that many of the words in the resource did not previously exist in te reo Māori. Hēmi

\footnotetext{
${ }^{78}$ The English language and the Māori language.
} 
decided to create new words in collaboration with other translators, however there was not enough time to consult with my community partners or wider rainbow communities about these new translations before the resource launch. When Hēmi sent me the completed list of words there was some concern among my Māori community advisors that these words would be taken up and used as official translations, rather than considered as one possible translation. To pre-empt this, we wrote a short paragraph introducing the te reo Māori translation (Te Tautoko i te Hunga Āniwawa o Aotearoa: He Puka Whaitake mā Ngā Mātanga Hauora Hinengaro):

A challenge we encountered during the translation process was that many of the words in this resource did not previously exist in te reo Māori, as the kupu 'takatāpui' has always held a space for all Māori of diverse genders, sexualities, and sex characteristics. We want to emphasise that the kupu we introduce here describe the essence of western understandings of sexuality and gender - in te ao Māori terms are not broken down like they are in te ao Pākehāa. There isn't yet a consensus among the Màori people involved in this project about the best way to translate many kupu in this resource, so we see this document as a starting point for a wider kōrero around creating new kupu for sex, sexuality, and gender terms.

We also decided against publishing a glossary of terms that outlined English terms and their Māori translation, and asked Hēmi to delay adding these new words to the Māori online dictionary or other official sources pending further consultation with wider Māori rainbow communities.

When Gender Minorities Aotearoa heard of our Māori translation, they were excited about the prospect of presenting the resource in a number of different languages and arranged funding for the resource to be translated into Chinese Mandarin. This translation was completed by Jiabao Zhao. Jiabao made the decision to leave some words in English (e.g. "demisexual" and "homoromantic"). I also created a large English print version which did not contain any design or illustration elements to be accessible for those who were reading on screen readers or found the designed version difficult to read due to the small print or busy design. 


\section{Accompanying Posters}

Given that the resource recommended that mental health professionals display visual signs of support in their service, we decided also to create a series of posters to accompany the resource. These posters welcomed rainbow clients into mental health settings, invited clients to share their pronouns, and invited clients to share feedback about how they could be better supported. The posters could be displayed in waiting rooms, in mental health professionals' therapy rooms, or on office doors. We also created a poster that shared top tips from the resource for supporting rainbow people in mental health settings, which I imagined mental health professionals could display in their staff rooms or other communal spaces. Although the posters were designed for mental health settings they could also be used in a wide range of other settings, including schools, workplaces, universities, and community organisations.

\section{Resource Launch and Distribution}

We held two events to launch the resource: one at Victoria University in Wellington, and one at RainbowYOUTH in Auckland. At these events we created a space for those who had been involved at any stage of the project, or who were interested in or supported the project, to come together and celebrate the completion of the resource. The resource launches were well-attended, with approximately 100 people coming along in Wellington and around 40 in Auckland.

Our resource website was made live on August $2^{\text {nd }}, 2019$. Visitors to https://rainbowmentalhealth.nz could (and still can!) read the resource online, download the resource in English, te reo Māori, Chinese Mandarin, or large print, and download posters designed to create a rainbow-friendly space. We also created an order form on the website where mental health professionals could order physical copies of the resource. At the time of writing, we have distributed approximately nearly 1000 resources to wide range of difference people and services around Aotearoa.

\section{The Resource}

This chapter concludes with the English version of our finished resource Supporting Aotearoa's Rainbow People: A Practical Guide for Mental Health Professionals, as well as the accompanying posters (Figures 21-24). 


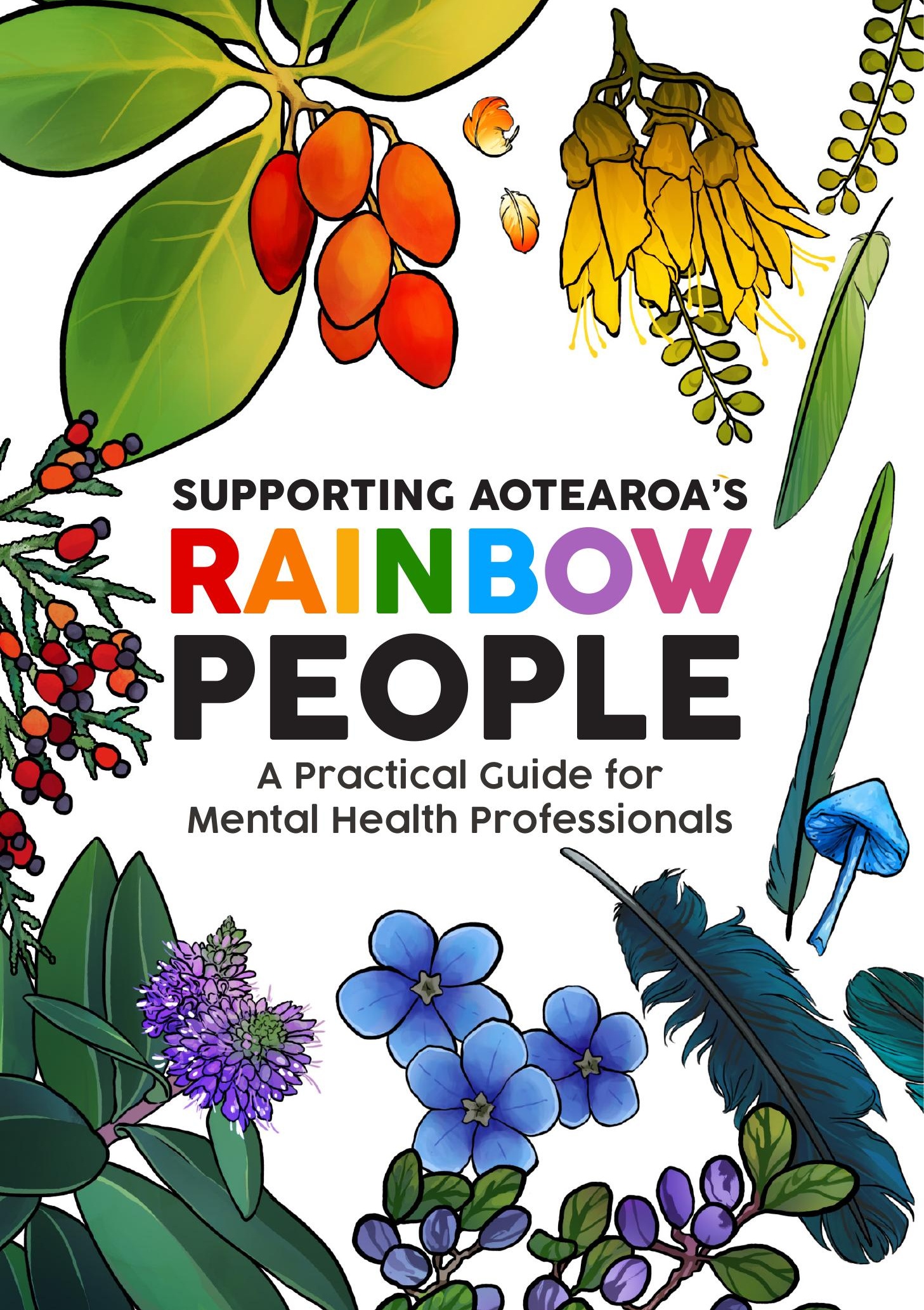




\section{our resource on supporting rainbow people in Aotearoa's mental health settings!}

\section{Who is this guide for?}

This guide is for anyone who provides mental health support in Aotearoa, including (but not limited to) counsellors, psychologists, psychiatrists, psychotherapists, tohunga (Māori healers), social workers, mental health nurses, and GPs. It will also be helpful for youth workers, group facilitators, and peer supporters.

\section{Why do you need this guide?}

Wherever you work in mental health, you work with rainbow people. Rainbow people in New Zealand face a range of complex issues, and mental health professionals don't always receive specific training about their needs and experiences. You already have the skills to provide great support - this guide is designed to give you information to build on and strengthen those skills.

\section{Who developed this guide?}

All the information you'll find here is based on findings from the Rainbow Mental Health Support Experiences Study and the Out Loud Aotearoa Project. Together, these projects involved interviews and surveys with, and submissions from, more than 1600 rainbow people in Aotearoa. To create this resource, researchers from Victoria University of Wellington's Youth Wellbeing Study teamed up with RainbowYOUTH, InsideOUT, and Gender Minorities Aotearoa. We also refer to other research studies throughout the guide, as cited on pages 52 and 53.

\section{Ngā mihi}

E hara taku toa i te toa takitahi, engari he toa takitini.

We want to give our sincere thanks to everyone who took part in the research that made this resource possible, and to everyone who gave us feedback to improve the resource. Ngā mihi aroha ki a koutou.

Resource design and illustration by Bo Moore (bomoore.net).
Throughout this guide, we'll share quotes from people in the rainbow community. We will use different birds to do this instead of using real names.

We use the umbrella terms 'rainbow people' and 'sex, sexuality, and gender diverse people' throughout this guide.

You might have heard different terms for this group, like LGBT or LGBTQIA+. We aim to be as inclusive as possible with our use of language but acknowledge that the terms we use don't work

$$
\text { for everyone. }
$$<smiles>C1CCC1</smiles>

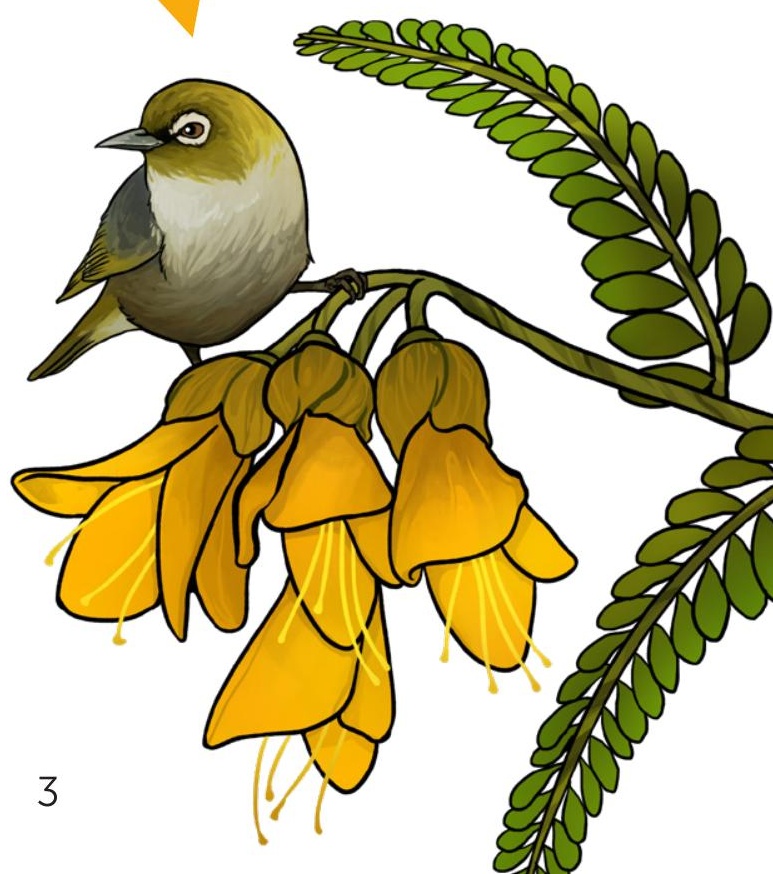




\section{Contents}

5 Our Kaupapa (Core Principles)

\section{Background}

Terminology

Historical and Contemporary Context

Intersections of Identity and Mental Health

Common Topics of Discussion in Therapy

Accessing Mental Health Support as a Rainbow Person

\section{Practice Guide: Supporting Rainbow Clients}

Self-Reflection on Identity

24 Affirmative Therapy

26 Asking About Identity

27 Creating a Supportive Therapeutic Space

29 Making Your Service Rainbow Friendly

30 Finding the Right Balance

31 Common Missteps - and How to Avoid Them

\section{Understanding the Diversity of Experiences Among Rainbow People}

33 Gender Norms and Rainbow Identities

34 Transgender People and Gender-Affirming Healthcare

38 Takatāpui People

39 Pasifika Rainbow People

40 Multiple Gender Attracted People

42 Intersex/VSC People

43 Asexual People

44 Non-monogamous People

45 Rainbow People with Disabilities

46 Rainbow Refugees and Asylum Seekers

48 Strengths and Resilience

51 Further Reading

52 References

54 Rainbow Organisations in Aotearoa

\section{Our Kaupapa (Core Principles)}

The information in this resource is based on five guiding principles. When supporting rainbow people in mental health settings:

\section{Take an affirmative stance}

An affirmative stance embraces a positive view of rainbow identities and relationships and considers the impact of discrimination on the lives of sex, sexuality, and gender diverse clients.

\section{Respect self-determination}

This means respecting the identities of rainbow clients and the ways they express their identities, as well as following their lead in conversations about identity and mental health.

\section{Engage in self-reflection}

Reflecting on our own identities, privileges, and beliefs about sex, sexuality, and gender diversity is central to supporting rainbow clients. This is not a one-time activity, but an ongoing process.

\section{Acknowledge the diversity of rainbow people}

Rainbow people are often represented as a single homogenous group. Mental health professionals can challenge this by recognising the diversity of experiences and needs among rainbow people.

\section{Learn about rainbow experiences and needs}

This includes the common challenges faced by sex, sexuality, and gender diverse people, as well as their strengths and resilience. 


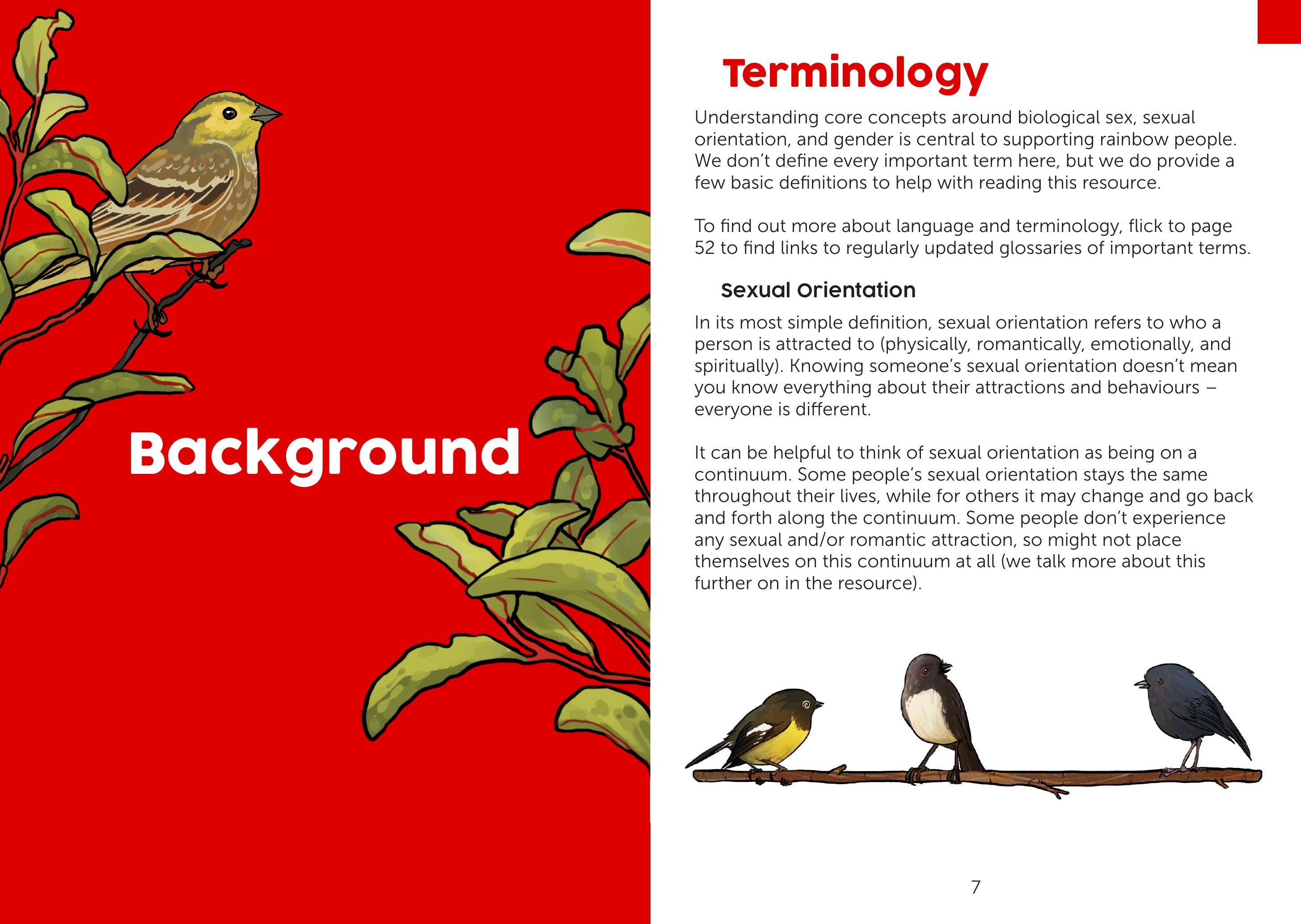




\section{Gender Identity, Expression, and Fluidity}

Gender is an aspect of who we are, how we describe ourselves, and how we express ourselves. For some people, their gender is the same throughout their life. For others gender is fluid, meaning it changes over time. Some people don't identify with any genders, and some people are questioning their gender. Gender expression is about how we present ourselves to the rest of the world - for example, our haircuts and the clothes we wear.

\section{Pronouns}

Pronouns are words used to refer to other people, as a substitute for their name. Commonly used pronouns are she/her/hers, he/him/his, and they/them/their, though there are many others. Use of they/them/their to refer to one person is grammatically correct. In te reo Māori, the third person pronoun is 'ia', and is used to refer to people of all genders.

\section{common Terms}

Transgender (trans) and gender diverse are umbrella terms for people whose gender is different from their assigned sex at birth. Cisgender (cis) is a term for people whose gender is the same as their assigned sex at birth. Agender is a term used for people who do not identify with any gender.

Nonbinary genders do not fit the man/woman gender binary. Like the term transgender, nonbinary can be an umbrella term. People under this umbrella may also describe themselves using one or more of a wide variety of terms (like genderqueer or genderfluid) or may simply use 'nonbinary' or 'nb'.

\section{Intersex/Variations in Sex Characteristics}

Biological sex is a label used to describe our physical bodies. Although most people are assigned male or female at birth, our sex characteristics (sexual anatomy, reproductive organs, hormonal patterns and/or chromosomal patterns) can be arranged in a multitude of different ways.

'Intersex' or 'variations in sex characteristics' are terms used when someone's sex characteristics are more diverse than the typical definitions of male and female. Intersex people use different terms to describe themselves, and it is estimated that up to $1.7 \%$ of the global population are born intersex, or have a variation in sex characteristics.

\section{Cender and Sexual Orientation among Intersex People}

There is huge diversity among intersex people in terms of gender. Many intersex people identify as male or female, others are nonbinary, others identify their gender as intersex, and others use some combination of these terms. There is also diversity in the way intersex people identify and express their sexual orientation.

\section{Diagnoses}

While some people may never know that they are intersex, many intersex people are diagnosed with an intersex variation, and that diagnosis could take place at many points throughout their life. There are over 40 intersex variations. Having a diagnosis is important for many intersex people, but there is a move away from seeing intersex as a disorder or something to be fixed. Instead, there is a shift toward embracing variations in sex characteristics as part of human diversity. 


\section{Top Terminology Tips}

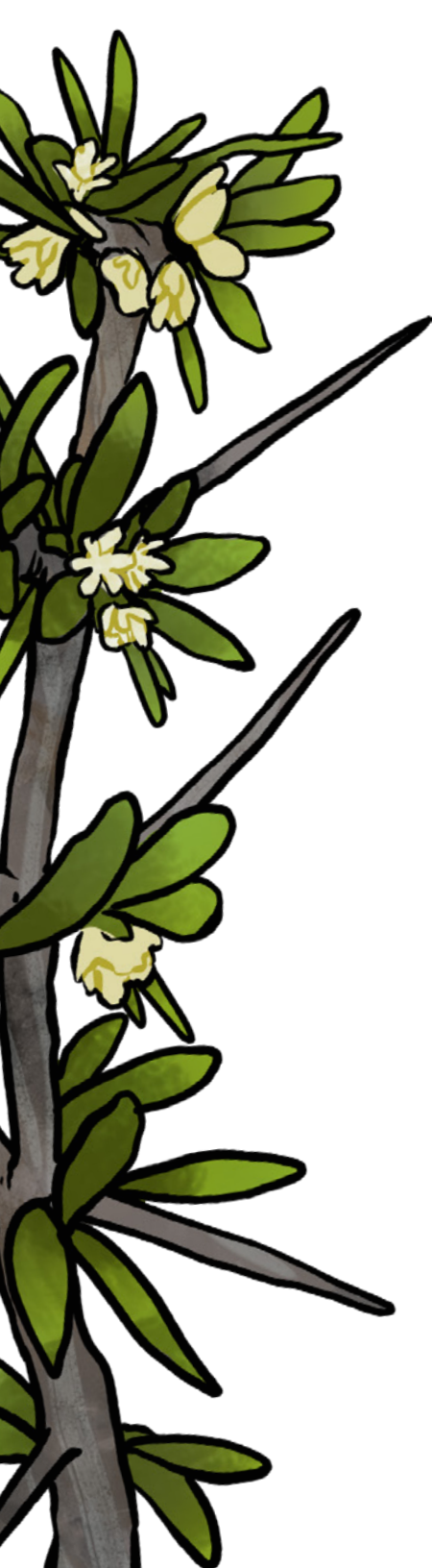

- Use the same terms that a client uses to describe themselves. Rainbow people frequently have their identities undermined and questioned in their daily lives, and mental health settings have the potential to be validating and healing environments where clients can explore and freely be themselves.

- There are no rules or criteria for using any identity label (e.g. transgender, bisexual, queer) - the important thing is that the term feels right for the person using it.

- If a client uses a term you're not familiar with, you can stop and check in on what the term means for them. Having some background knowledge of terminology is helpful however, as it can be frustrating for clients to spend time educating mental health professionals about language and terminology.

- Be mindful that people often use different identity labels in different situations or with different people, or they might use a label for simplicity. For example, someone might describe themselves as 'queer' among friends, but use the term 'bisexual' at work.

- Lots of mental health professionals worry about getting terminology wrong, especially if it's unfamiliar. Remember that everyone makes mistakes - if you do, just apologise and move on.
"Our linguistic choices are crucial, particularly as minorities. That's kind of how we relate to the world, by choosing certain words to describe us, our behaviours, our histories, whatever. It's really important to honour pronouns and names - if I call myself transgender, don't call me a transsexual. Our word choices are deliberate and I don't like seeing other people mess with those."

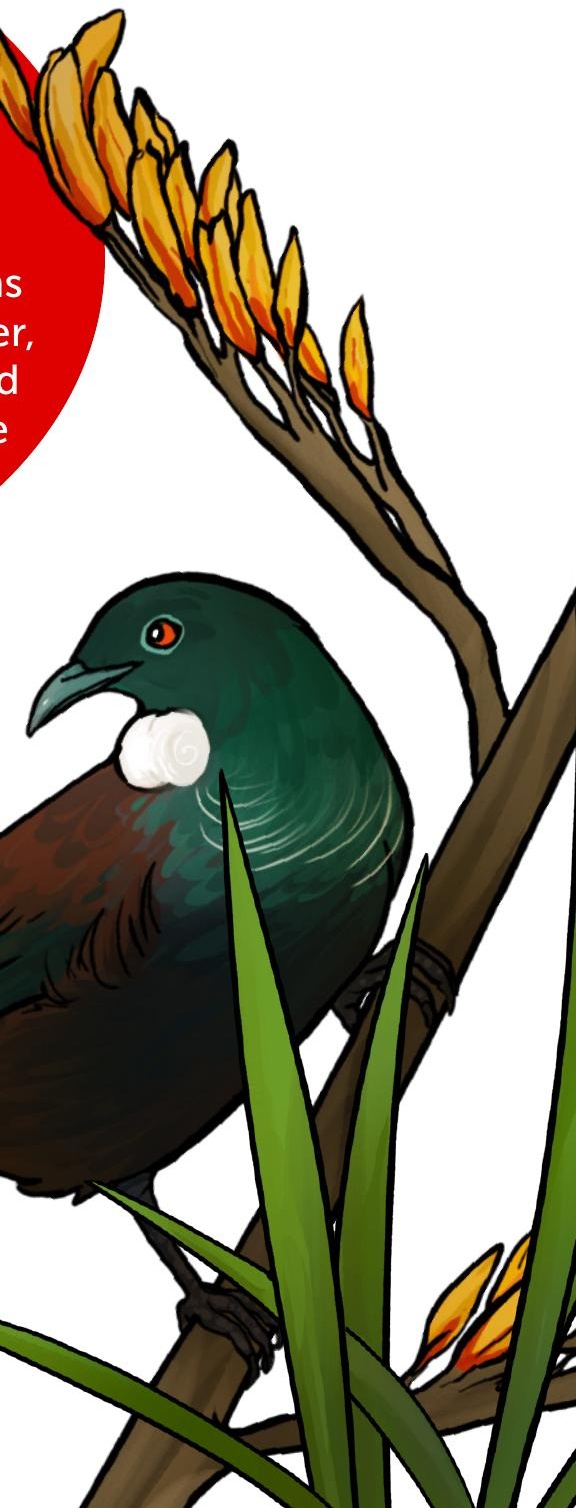




\section{Historical Context}

\section{Takatāpui and Pasifika Rainbow Identities}

Pre-colonisation, sex, sexuality, and gender diversity was accepted and valued throughout Pacific cultures, including in te ao Māori (the Māori world), with takatāpui (Māori of diverse gender identities and sexualities) claiming their identity through whakapapa, or genealogy. The colonisation of the various islands in the Pacific by European settlers disrupted this, bringing Victorian and Christian attitudes to gender roles, morality and sexuality. Colonisation throughout the Pacific also brought British laws that denied the basic human rights of rainbow people.

\section{Colonial Law}

Under British law, sexual relations between men were criminalised and came with harsh punishments. Sex between women has never been criminalised in New Zealand, though women who engaged in 'inappropriate sexual practices' could be sent to government institutions or committed for psychiatric treatment. The 1970s saw the birth of the modern gay and lesbian rights movement in New Zealand, with the formation of Gay Liberation groups. Significant law reform followed, including the decriminalisation of homosexuality in 1986 and the introduction of marriage equality in 2013.

\section{Trans and Intersex Histories}

For most of New Zealand's post-colonial history, there has been widespread silence about the social and legal status of trans and intersex people. Trans people weren't able to legally change their sex until 1995, and have experienced discrimination in housing, work, and public life. Trans people have also struggled to access essential healthcare services. Intersex people have faced a lack of recognition of their existence and needs, and for the last half-century, medical interventions have been performed in New Zealand on intersex babies, meaning they have no opportunity to consent.

\section{Contemporary Context}

Despite legislative gains for rainbow rights in New Zealand and increasing societal acceptance, rainbow people continue to experience widespread stigma and discrimination. Sometimes this is explicit, including verbal, physical, and sexual assault and harassment. At other times, it is more subtle.

\section{Coming out - or Staying In!}

Due to widespread assumptions that everyone is straight, cisgender, and not intersex, rainbow people are often expected to 'come out' about their identity in a way others are not.

\section{Misconceptions and Assumptions}

Rainbow people also face common misconceptions or assumptions that their identities are a phase, a choice, or not valid, and that everyone fits neatly within the gender binary.

\section{Healthcare}

Gender diverse people face significant barriers to accessing gender-affirming healthcare in New Zealand, and 'normalising' medical interventions on intersex babies are still legal and practised here in Aotearoa.

\section{Diversity of Discrimination}

For some rainbow people, homophobia, transphobia, and/or intersex discrimination is part of daily life and has profound negative impacts on their health and wellbeing. Other rainbow people are affirmed and validated by those around them, and many people's experience is somewhere in between these two ends of the spectrum. It is key to keep this diversity of experience in mind when working with rainbow clients. 


\section{Intersections of Identity}

"Our gender expression changes the way that we're treated in society.

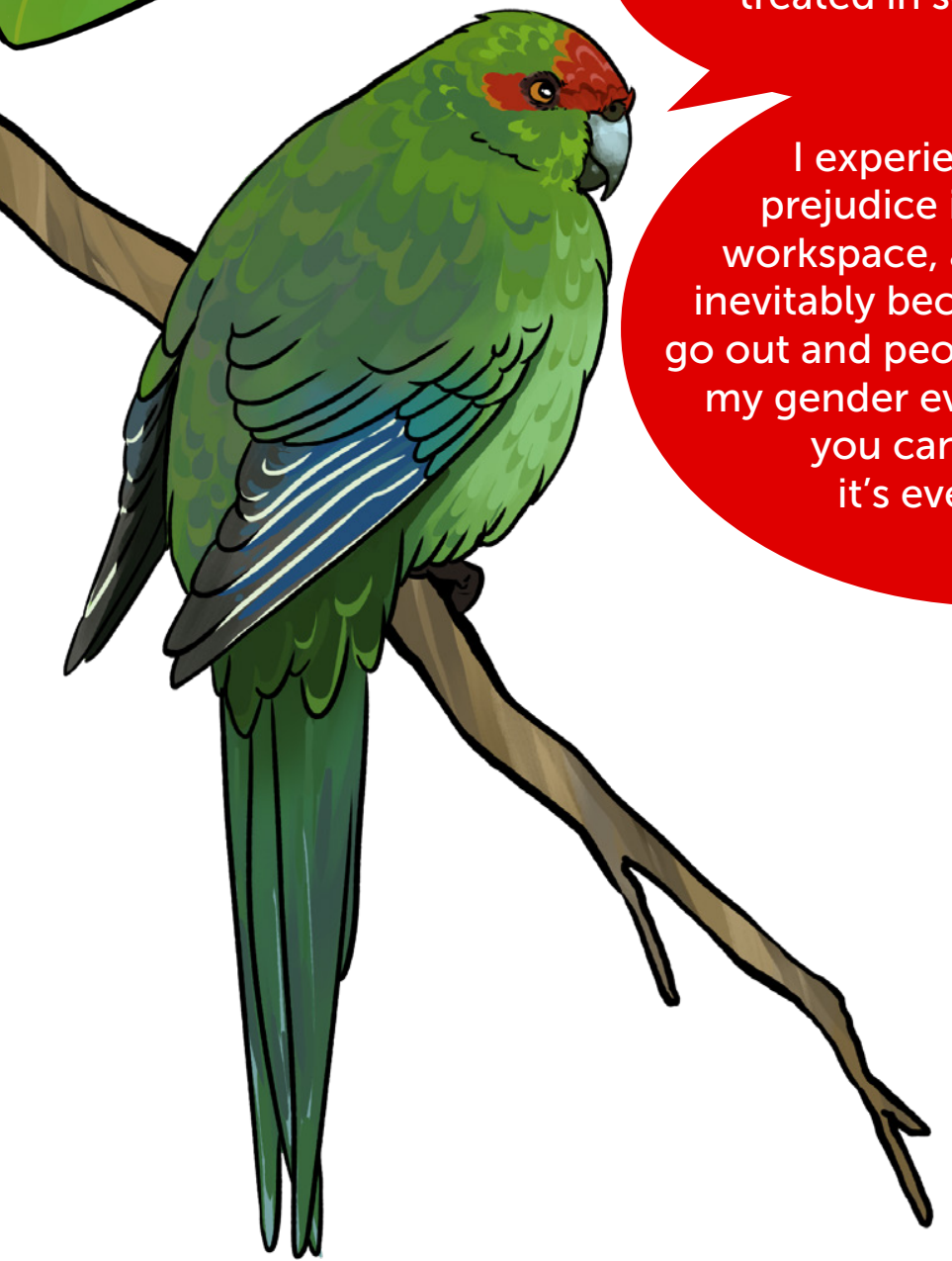

Intersectionality is a framework we can use to understand the ways in which sexual orientation, gender, and biological sex intersect with a person's other important identities.

Intersectionality states that oppressions based on different social categories (e.g. sexual orientation, gender, ethnicity, disability, education, age, and class) are interlinked, and cannot be addressed separately from one another. Holding more than one identity means that the effects of oppression are compounded - the negative impacts of these oppressions on a group or an individual are amplified when experienced together.

While sex, sexuality, and gender diverse clients are all exposed to potential discrimination based on their rainbow identities, they are impacted by other forms of discrimination in different ways. Many of our clients may face multiple, overlapping oppressions, so discussing structures like colonisation, racism, ableism, sexism, and classism is just as important as exploring homophobia, transphobia, and intersex discrimination.

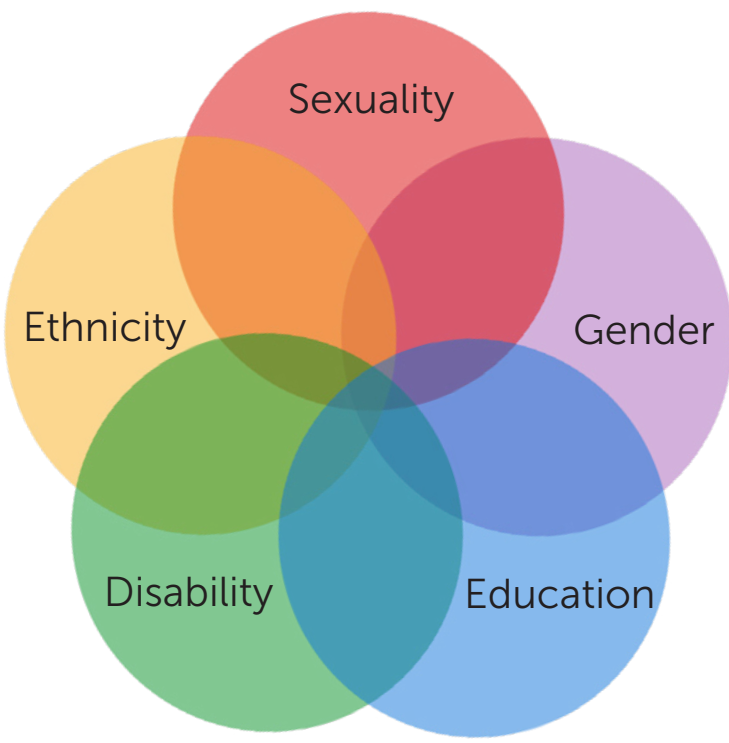




\section{Rainbow Identities and Mental Health}

\section{History of Rainbow Identities in Mental Health}

Rainbow identities were historically pathologised in the field of mental health care; homosexuality was included in the Diagnostic and Statistical Manual of Mental Disorders (the DSM) in various forms until 1987, and gender diversity carried a 'disorder' label until 2013. Gender dysphoria (the distress associated with the disconnection between one's body and sense of self) is still in the DSM as of 2019 (this is called 'gender incongruence' in the International Classification of Diseases-11). Many people have called for it to be removed, but others are concerned that this would threaten gender diverse people's access to healthcare and support services.

Because of this pathologising history, many rainbow people feel mistrust towards the field of mental health care and are often hypervigilant to signs of pathologisation in mental health settings.

\section{Minority Stress}

High rates of mental health difficulties among rainbow people are now understood using the minority stress model. Put simply, the model states that the external world affects the internal world - societal stigma and discrimination create a hostile and stressful environment for sex, sexuality, and gender diverse people.

This stress, in turn, increases the risk for mental health problems. The minority stress model is supported by a large body of research, which suggests that in order to address the high rates of mental health problems among rainbow people, we need to intervene at two levels - at the societal level, to reduce stigma and discrimination, and at the individual level, to support sex, sexuality, and gender diverse people as they face stress and adversity.

\section{Common Topics of Discussion in Therapy}

\section{Rainbow People and Intimate Relationships}

It's important to remember that not all clients are in relationships, have been in relationships, or want to be in relationships. A person's experience with relationships might not seem to correspond to their sexual orientation (for example, a person who identifies as bisexual might not have had relationships or sexual experiences with more than one gender). This doesn't mean that their identity can be questioned by anyone but themselves. Another consideration is that New Zealand's rainbow communities are small and interconnected, which can complicate intimate relationships and friendships.

\section{Coping with Societal Stigma and Discrimination}

As mentioned earlier in this resource, there are widespread misconceptions that societal stigma and discrimination towards rainbow people ended with legal reforms like marriage equality. Subtle forms of discrimination (sometimes called microaggressions, e.g. unkind jokes, comments, and looks) can be especially hard to pinpoint and challenge but can build up and cause significant distress. Rainbow clients may want to talk through experiences of discrimination, or worries about discrimination, in mental health support settings. 


\section{Sharing about Identity}

The process of understanding one's own identity and sharing aspects of identity with others is often called 'coming out' or 'disclosure'. This can be a difficult process, especially if someone is worried that those around them won't be accepting. Sharing about identity can also be freeing, allowing someone to live in a way true to who they are.

Some important things to know about coming out:

- Coming out is not a one-time thing. Many rainbow people come out on a daily basis, or many times in a single day.

- People might be out to different extents in different parts of their life. For example, someone might tell their family and friends that they're nonbinary, but not be out as nonbinary at work.

- The concept of coming out doesn't make sense to everyone. For many people, not sharing about their rainbow identity doesn't make them 'closeted' - it's just a personal choice.

- Rainbow people don't need to be out to everyone they know in order to be happy. Some people might choose not to come out to others because they feel their identity is personal information, not relevant to their relationship with that person, or because it might not be safe. Often, choosing whether to share about identity involves balancing these different considerations.

- If someone comes out to you, you can thank them for trusting you with that. You can also support clients by providing space and time, and by acting as an affirming sounding board during this process.

When talking about coming out, mental health professionals should go at the client's pace and not idealise coming out as an end goal for the client in terms of improving their mental health and wellbeing.

\section{Children and Parenting}

Some rainbow clients might want to talk about parenting and children with their mental health professional. Some people feel intense grief if they are not able to 'traditionally' conceive a child with their partner, and others worry that it will be difficult to have children through methods like adoption, surrogacy, or IVF. Rainbow parents are also faced with mis-conceptions that their children will be somehow harmed by their parents' identities, even though research has proven otherwise. Parents may also access mental health care to ask how they can best support their rainbow children.

\section{Internalised Negative Attitudes}

Some rainbow people internalise negative societal attitudes about sex, sexuality, and gender diversity, and feel shame and loathing about their own identity. Those with internalised negative attitudes may reject their identity, or want to change it. Here, it's important that mental health professionals maintain their affirmative stance and support the client to explore where their negative attitudes might have come from.

\section{Gender-Affirming Healthcare}

Gender-affirming healthcare is any healthcare that affirms or validates someone's gender, including transition-related services and therapy that supports people through the process of transition. Gender diverse clients in need of gender-affirming healthcare often require a letter of referral from a mental health professional before they can access the care they need. Not all mental health professionals are able to provide this referral, so will sometimes need to direct the client to someone who can. Mental health professionals can also support clients who are figuring out what kind of healthcare they need. You'll find more information about gender-affirming healthcare on page 36. 


\section{Affirmative Therapy}

Affirmative therapy is an approach that embraces a positive view of rainbow identities and relationships and considers the impact of stigma and discrimination on the lives of sex, sexuality, and gender diverse clients.

Central to affirmative therapy is affirmation and validation of rainbow identities.

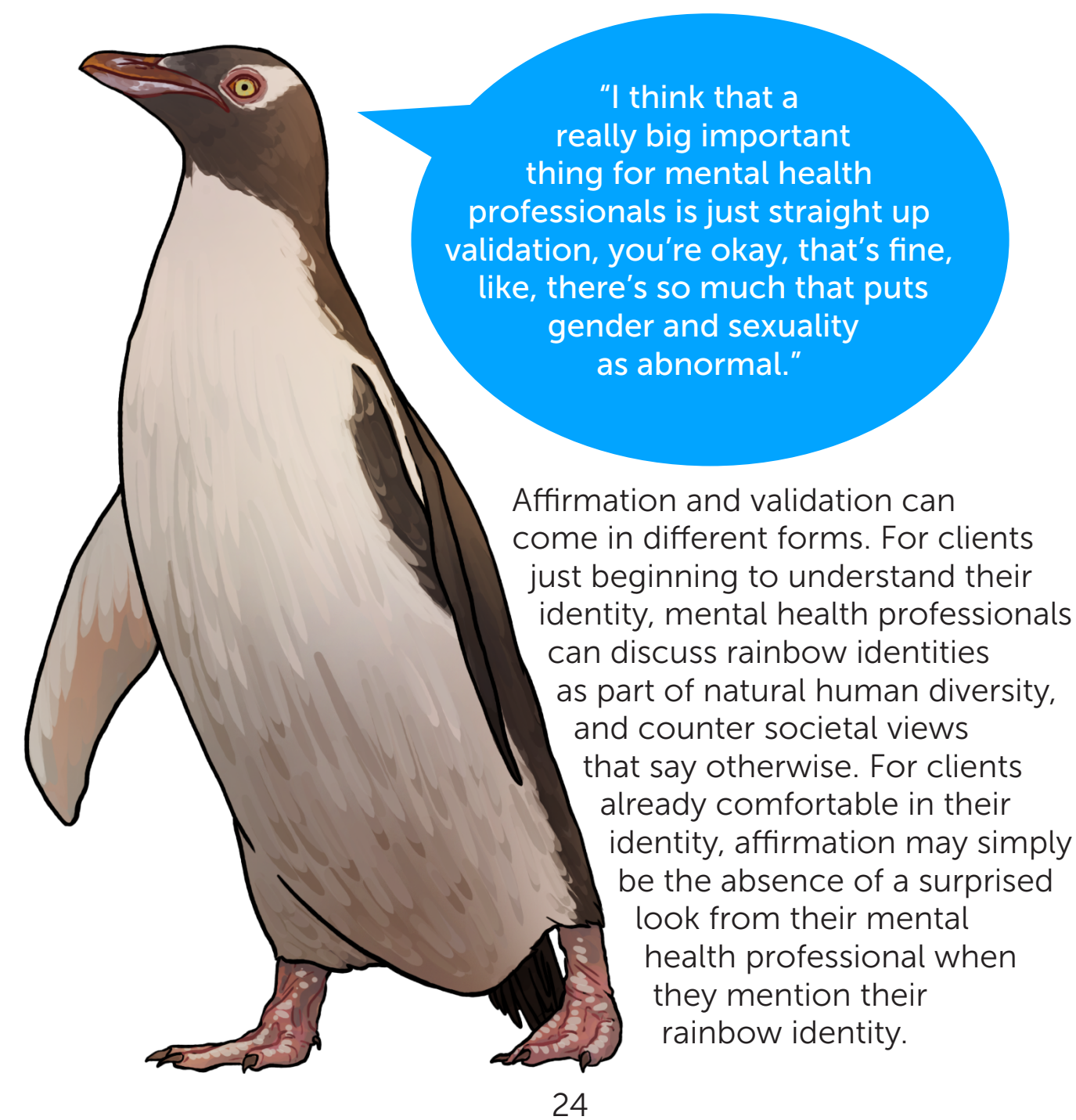

Some mental health professionals wonder whether there is a need for affirmative therapy - after all, isn't all of our therapy affirmative?

Research with rainbow people shows that standard therapeutic practices can maintain societal silence around sex, sexuality, and gender diversity. Mental health professionals rarely initiate conversations about sex, sexuality, and gender in therapy, instead leaving it up to their clients. Although this is usually done with the intention of being client-centred, rainbow clients often take this silence to mean that mental health professionals have assumed their identity. Silence around sex, sexuality, and gender can also signal that these topics are off limits for discussion, especially when the mental health professional asks about all other important aspects of life.

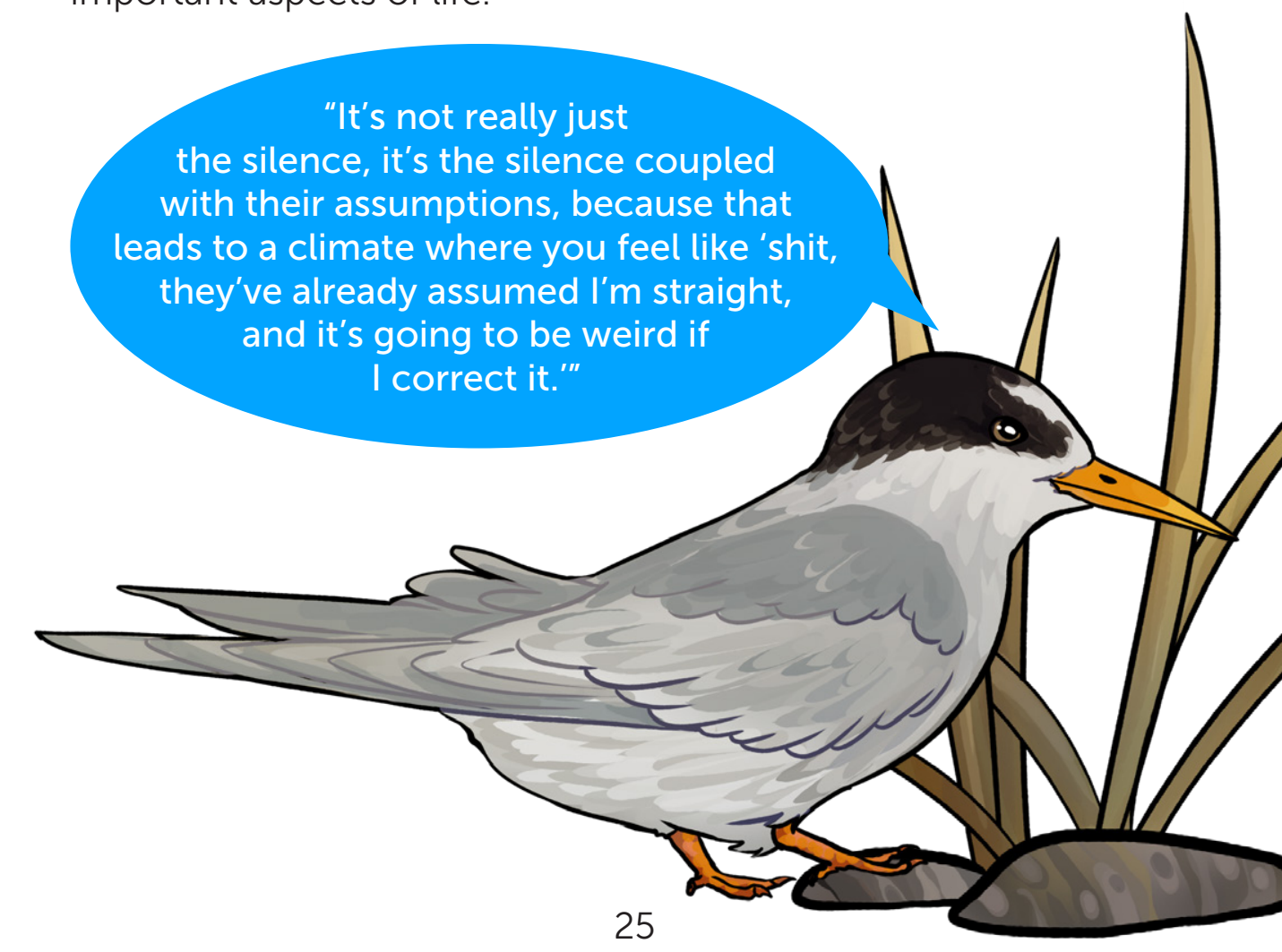




\section{Asking About Identity}

There are many different ways that mental health professionals can challenge the societal silence about sex, sexuality, and gender. Perhaps the most obvious way is to ask clients how they identify during an assessment, or the 'get to know you' part of the session.

Asking about sex, sexual orientation, and gender is a contentious issue. For some clients, it shows that their mental health professional hasn't assumed their identity, and creates a space for them to talk about identity. For others, being asked about how they identify in a mental health setting can feel confronting.

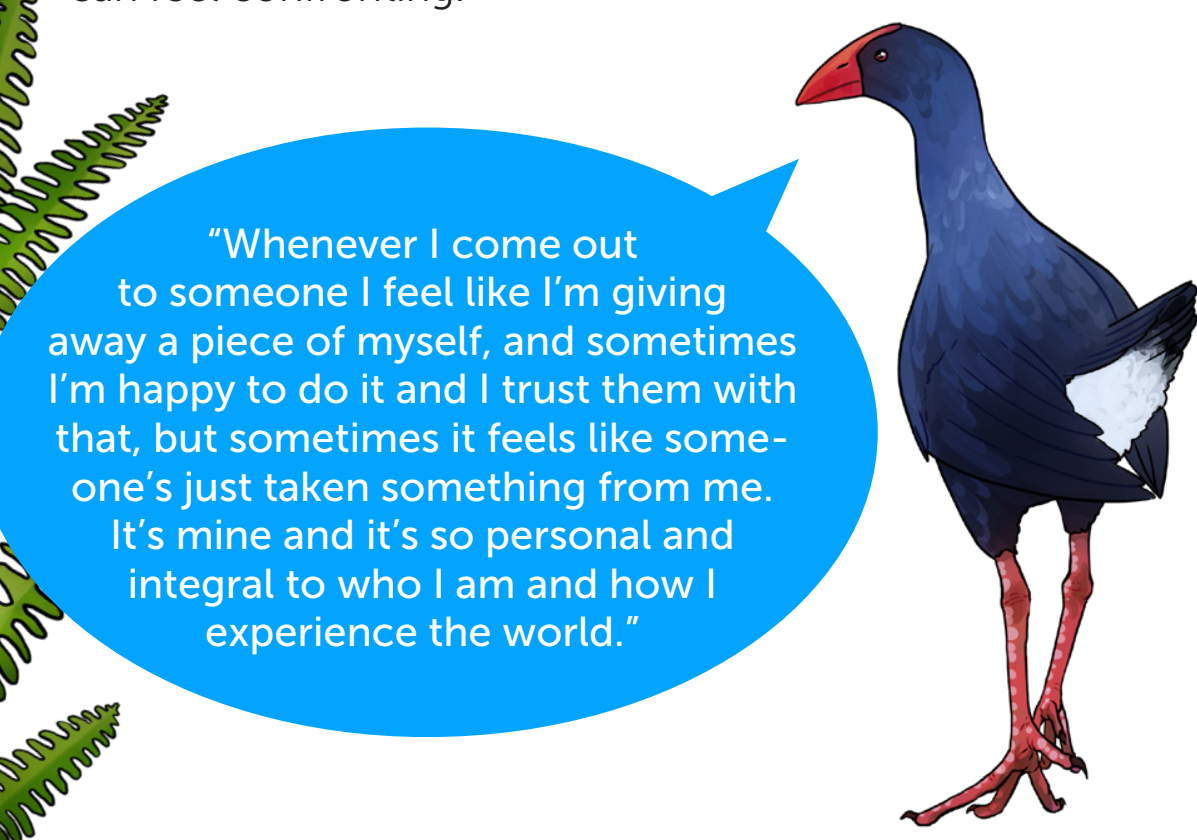

An alternative to directly asking about identity is to bring up sex, sexuality, and gender as a potential topic the client might want to talk about, without pressure to do so at a particular time. For example, you could say, 'Is sex, sexuality, or gender something you want to talk about here?'

\section{Creating a Supportive Therapeutic space}

\section{Expansive Language}

One of the easiest and most helpful things we can do to signal to clients that we are rainbow friendly is using expansive language. Expansive language refers to any language that is deliberately open or vague so as to not assume someone's identity or narrow their experiences. For example, rather than asking if a client has a boyfriend or girlfriend we can ask:
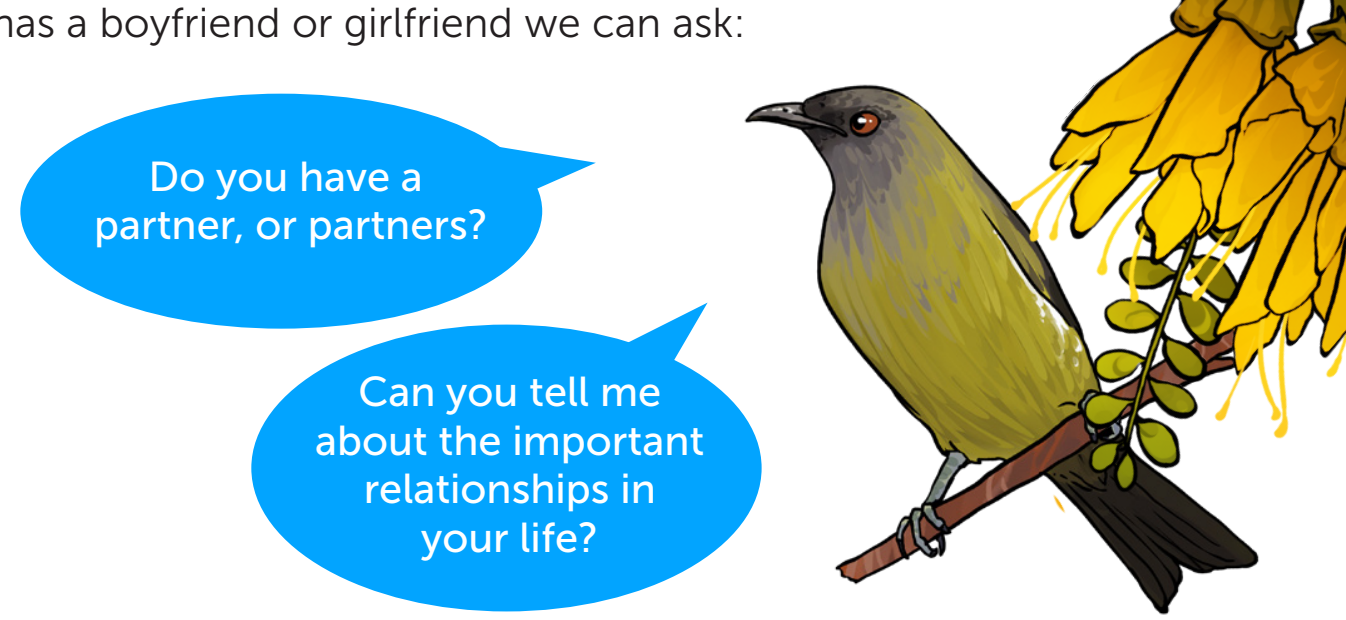

Using expansive language means referring to anyone using they/them pronouns (known as gender-neutral pronouns) or a person's name until we hear more about them or learn the pronouns they prefer.

\section{Maintaining Privacy}

When talking to rainbow clients about their names and pronouns, be sure to check in about whether they have a preferred way of being publicly contacted or addressed, for example when being called in the waiting room or sent any official communications. You should also ask what name and pronouns clients use around their family/whānau, at school or work, and in other important areas of life. 


\section{Sharing and Checking Pronouns}

A great rainbow-friendly practice is to share our own pronouns when we introduce ourselves and ask our clients what pronouns they use. Some might never have heard of pronouns, and this is a great opportunity to spread the word. When asking about pronouns make sure to ask all clients, rather than only asking clients that you think might be rainbow - when pronouns are asked of some people and not others, clients may feel they have been singled out as trans.

Alternatives to directly asking about pronouns in person include asking clients how they would like to be referred to or including a pronoun question on intake or registration forms. Just make sure you have some examples of pronouns (e.g. he/him, she/her, they/them) to make it clear what you're asking, and leave an open box. You can also share your pronouns by including them in your email signature, or on ID cards and name badges.

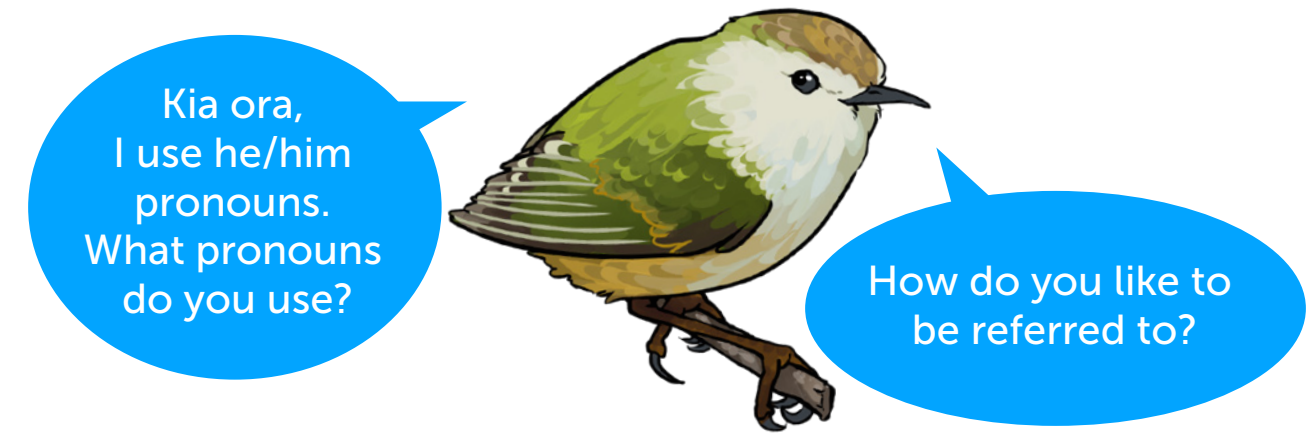

When sharing and checking pronouns, let your client know that they can update you any time their pronouns change. You can also update your clients' gender on the National Health Index to reflect their identity, even if their legal name or gender marker has not been changed (if you do not have access to make these changes, ask your administrative staff or contact the Ministry of Health). This can be particularly useful for refugees, asylum seekers, or other migrants who cannot amend these details on official NZ documents until they are permanent residents.

\section{Making Your Service Rainbow Friendly}

Creating a friendly space for rainbow clients must be done at a wider institutional/organisational level, as well in our personal practice. This inclusivity can make a big difference in how comfortable rainbow clients feel meeting a mental health professional for the first time.

\section{Bathrooms}

Every mental health service should have at least one accessible all-gender or gender-neutral bathroom that anyone can use. This acknowledges that not all clients are men or women, and is important for those who may not feel safe or comfortable using either the men's or women's bathrooms. Often this can be as simple as changing a sign.

\section{Forms}

Any form used in your service should ask about gender in an inclusive way. This means providing more flexibility than two tick boxes marked male and female. The easiest way to do this is with a single open-ended box, but if you must use closed boxes, include options like 'gender diverse', 'trans man', 'trans woman', 'takatāpui' and 'nonbinary'. Make sure there's at least one write-in space, and that people can tick multiple options.

You should also consider whether you need to know about clients' gender, the sex marked on their birth certificate, or both. Make this clear to help clients fill out forms easily and accurately.

\section{Visual Signs}

Many rainbow people find visual signs of support helpful. This could be a rainbow and/or trans flag in your waiting room, or posters and flyers that include people of diverse sex characteristics, genders, and sexual orientations. 


\section{Finding the Right Balance}

For some people, their rainbow identity will be a central focus in their sessions with you but for others, it may not be relevant to why they've accessed support. Supporting rainbow clients is about finding a balance between creating space to talk about identity, but only making it the focus when relevant.

"If I had a mental health specialist that kept on coming back to my gender or sexuality, I would probably feel quite weird about that unless it was very clearly the source of my [distress], which it might be for whatever reason."

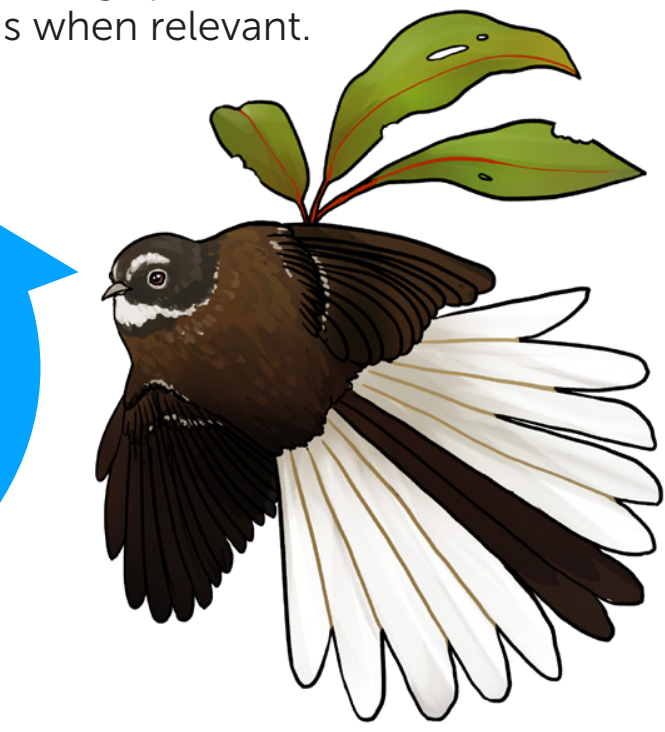

Rainbow clients are generally happy to be asked about whether they see their identity as impacting on their mental health, or whether they want to talk about sex, sexuality, or gender in therapy. It is important to think about how to approach questions surrounding identity. There's a difference between suggesting that someone's identity caused their mental health difficulty and questioning whether the stress that can come with discrimination and exclusion is having an impact on their mental health and wellbeing.

\section{Background Knowledge}

Having background knowledge about sex, sexuality, and gender diversity is essential. It's just as important to balance that with getting to know the client in front of you. If we rely on our background knowledge too much, we can make incorrect assumptions about what's happening for our clients.

\section{Common Missteps for Mental Health Professionals..}

\section{... And How to Avoid Them!}

Trying to find a cause or explanation for someone's identity, e.g. suggesting childhood trauma caused someone's rainbow identity, or asking about signs of rainbow identities in childhood.

We usually look for the cause of things we think are unusual or want to change. Identity isn't one of those things, so there's no need for us to figure out where our clients' identities came from (plus - it's impossible!).

Overidentifying with clients, e.g. talking about a friend, relative, or previous rainbow client when it's not relevant. This can make clients feel lumped in with other people with whom they may share nothing in common.

This is often done to show that someone is rainbow friendly, but there are lots of other ways you can do this (and many are included in this resource)

Assuming someone's identity based on their name, how they look, and/or what they talk about in therapy.

Use expansive language, share the pronouns you use, and check clients' pronouns (see page 22).

Getting an incomplete picture of the structural factors impacting on a client's life (e.g. racism, sexism, ableism, homophobia, transphobia, and intersex discrimination).

When clients don't have space to talk about oppression, this recreates that oppression in mental health settings. Mental health professionals can ask open questions about whether any of these factors are impacting on clients' lives or mental health. 


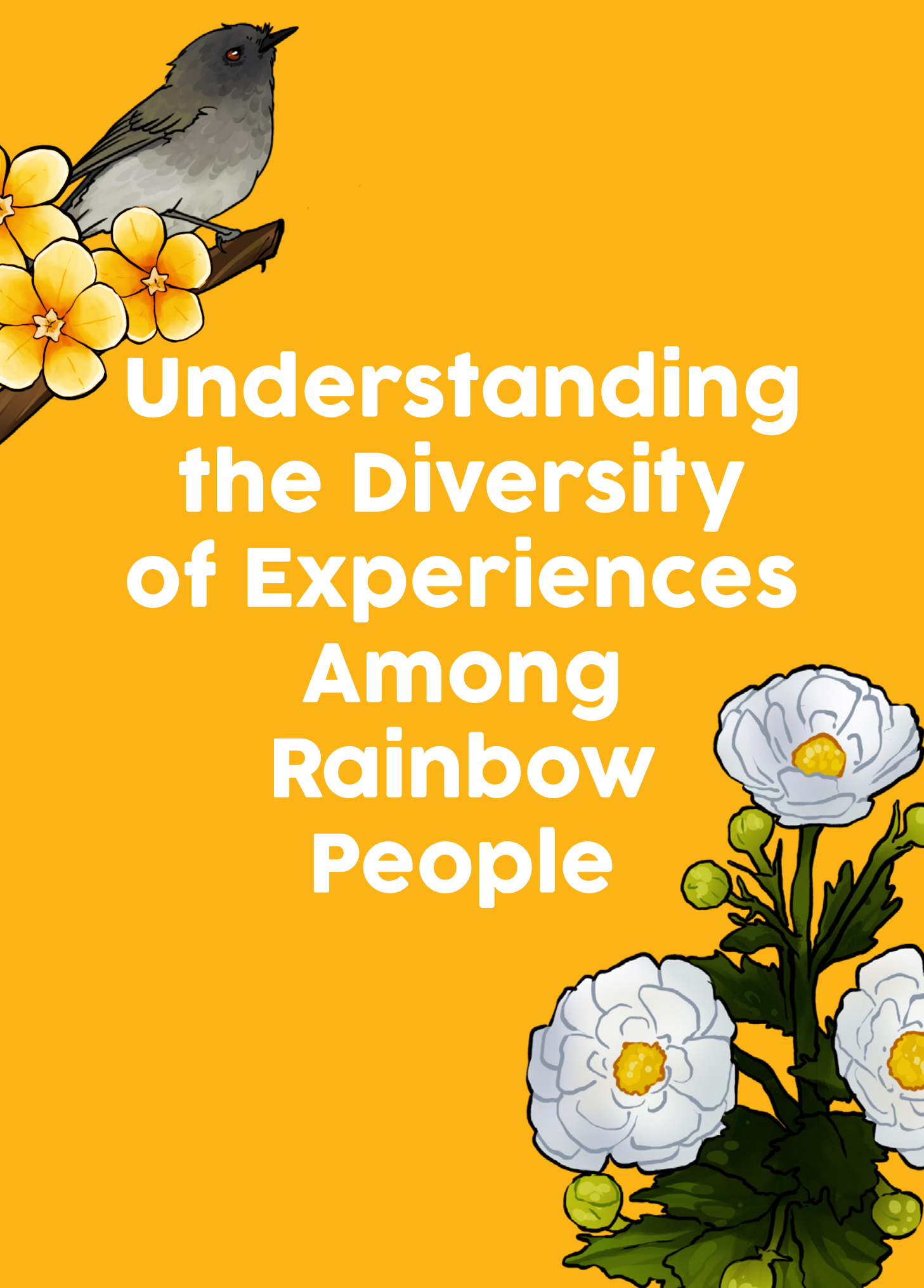

\section{Gender Norms and Rainbow Identities}

Gender norms and stereotypes affect rainbow people in different ways. Below we outline a few of the more common stereotypes around gender norms within rainbow identities, but these examples are by no means exhaustive.

- Rainbow women face sexism and misogyny as well as discrimination based on their rainbow identity. For some women, this leads to invisibility, where their peers and healthcare providers assume that they are heterosexual, and ignore their needs and experiences. Other women (e.g. transgender women) tend to be hypervisible - often they are recognised and have their right to privacy eroded in everyday circumstances, leading to high levels of stress and hypervigilance. Hypervisibility can also increase the risk for physical and sexual violence.

- Lesbian women commonly face stereotypes that they are all masculine, that their relationships will follow the same 'model' as heterosexual relationships (you might have heard inappropriate questions like 'who wears the pants?' before), and that they date women because they aren't desirable to men or have experienced sexual harm from men.

- Gendered stereotypes can lead to widespread denial or minimising of domestic violence within rainbow relationships (i.e. the perception that woman against woman violence isn't as serious as male violence against women). Research indicates that rainbow couples experience domestic violence at similar rates to straight couples, but are less likely to report it.

- Men who date men can feel pressured to present their gender in a way that aligns with traditional gender norms (strong, competitive, and masculine). They are often negatively portrayed as weak, feminine, and passive by comparison. Rainbow men can face stigma and violence if they don't fit gender stereotypes, or ostracisation within rainbow communities if they do. In addition, rainbow men face harmful stereotypes of promiscuity and over-sexualisation. 


\section{Transgender People}

We noted earlier in this resource that transgender is an umbrella term for anyone whose gender is different from their assigned sex at birth. Transgender people are incredibly diverse, not only in terms of how they identify their gender but how they express and describe it.

\section{Gender Dysphoria}

Gender dysphoria (sometimes called 'gender incongruence') is the distress associated with the dissonance between someone's gender or personal sense of self, and their body. Many (but not all) trans people experience gender dysphoria, and the intensity of gender dysphoria can change over time. Some people who experience gender dysphoria describe hating their body. Others describe it as a sense of unease and restlessness that can't be shaken off, a feeling of being out of place, or an inability to relax. It's common for someone to say that they know something is wrong, but they can't put their finger on what it is. On the flip side of gender dysphoria is the happiness and comfort that comes with being gendered correctly or feeling like one's body fits with their sense of self - some people call this gender euphoria.

\section{Transitioning}

Transitioning is the process of moving from being seen as someone's assumed gender (usually the same as their assigned sex) to their selfidentified gender. There is no one way to transition. Transition can include:

- Social transition - using different names and pronouns, changing hairstyles and clothes, as well as binding breasts or wearing breast forms.

- Legal transition - changing legal names and gender markers on legal documents like passports.

- Medical transition - laser hair removal, hormone therapy, and various surgeries such as facial surgeries, top surgery (removing or augmenting breasts), hysterectomy, and bottom surgery (to alter genitals), which can also be called gender reassignment surgery or sex reassignment surgery.

\section{common Narratives about Transgender People...}

... And the Realify!

It is important that we challenge misconceptions about what it means to be transgender and communicate that there is no 'correct' way to be trans.

All transgender people know they are trans from an early age. Transgender people realise they are trans at all points along the lifespan.

All transgender people fit the gender binary (are either men or women). Many trans people are nonbinary, questioning their gender, or don't identify with any gender.

All transgender people present their gender in a traditional or stereotypical way (i.e. all trans men as hyper-masculine, all trans women as hyper-feminine). Trans people present and express their gender in a multitude of ways - just like cis people.

All transgender people need to transition using every medical, surgical, and social intervention available to them.

Transition is unique to each person, and not everyone will need or want all available interventions.

Transgender people's difficulties are always a product of low self-esteem and self-worth.

While self-compassion can be an important part of therapy for transgender clients, navigating a hostile environment requires more than just self-love.

When transgender people are experiencing gender dysphoria at the same time as mental health problems, the mental health problems should be addressed first.

Lack of access to gender-affirming healthcare exacerbates mental health problems, so gender-affirming healthcare and mental health support can (and should) be provided at the same time. 


\section{Gender-Affirming Healthcare}

Gender-affirming healthcare is any healthcare that affirms or validates someone's gender, including transition-related services and therapy that supports people through the process of transition. For those who need it, gender-affirming healthcare is essential for wellbeing. A large body of research shows that access to gender-affirming healthcare decreases psychological distress and gender dysphoria and increases quality of life (see page 53).

\section{Gender-Affirming Healthcare Provision in New Zealand}

- Gender-affirming healthcare is technically publicly funded. The Ministry of Health funds genital surgeries and local District Health Boards fund other medical procedures.

- Publicly funded services are often only available in some parts of the country, and in areas where the care is are available, waiting times often stretch to months or years.

- Historically, those in need of gender-affirming healthcare had to receive a 'gender identity disorder' diagnosis from a mental health professional in order to get that care. 'Gender identity disorder' has been replaced in the DSM with 'gender dysphoria.

- Currently, experiencing gender dysphoria is required in order to access gender-affirming healthcare, and mental health professionals are routinely asked to assess the client's experience of gender dysphoria.

\section{Informed Consent}

The ideal model for gender-affirming healthcare is the 'informed consent model' which respects transgender people's self-determination. This model requires that the service user understands the potential risks and benefits of treatment, the alternatives to treatment, and has the capacity to weigh these options.

\section{Myths about Gender-Affirming Healthcare...}

... And the Reality!

Part of an assessment for gender-affirming healthcare involves making sure the client is 'really transgender' (e.g. they have a history of gender dysphoria and a stable gender identity). Clients without this history should be given time to think.

There is no 'right' way to express gender, be transgender, or transition. It's also important to remember that barriers to care and long waiting times can increase distress and the risk for suicidality.

You can help the client by carrying out a holistic psychosocial assessment, linking the client in with other supports, and advocating for the client. The Aotearoa guidelines cited on page 53 provide information to guide this process.

Only health professionals can establish what kind of genderaffirming healthcare a transgender client needs.

Health professionals should trust the self-determination of an individual and that they know what's best for them when it comes to gender-affirming healthcare.

Many transgender young people 'grow out' of their transgender identity later in life, so health professionals should withhold access to gender-affirming healthcare until they are sure the client won't change their mind or regret it later on. Gender can be fluid, so a change in gender identity does not mean that a previous decision to get gender-affirming healthcare was wrong. On top of this, the vast majority of people who receive gender-affirming healthcare will not regret their decision to do so.

Rather than trying to establish that a client is 'really' transgender or will be forever, our role is to trust transgender clients when they tell us what they need. 


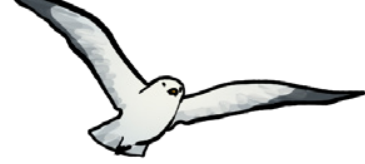

Takatāpui is a traditional Māori term meaning 'intimate companion of the same sex.' It has been reclaimed to refer to all Māori of diverse genders, sexualities, and sex characteristics.

\section{Identity and Whakapapa}

There are widespread misconceptions that rainbow identities are a 'Pākehā thing' or that sex, sexuality, and gender diversity was not part of the Māori world before colonisation. In fact, takatāpui have been always been a valued part of Māori communities, and stories of takatāpui identities and relationships have been told in waiata (songs), whakataukī (proverbs), and pūrākau (stories) for hundreds of years. The use of the word takatāpui inextricably links one's identity as Māori to their sex, gender, and/or sexuality, and many takatāpui are reassured to hear that they inherited their gender and sexuality from their tūpuna (ancestors).

\section{Discrimination, Support, and Disconnection}

Takatāpui may face discrimination based on their rainbow identity, as well as racism and the intergenerational trauma that comes with colonisation. Many takatāpui receive support and love from whānau, but others struggle with rejection from whānau and disconnection from hapū, iwi, and their wider Māori culture. Disconnection can be especially common among urban Māori, many of whom have never had the opportunity to visit their ancestral lands.

\section{Accessing Mental Health Support}

Many takatāpui feel pressured to pick one or other part of their identity when accessing mental health support. As mental health professionals, it is key that we understand takatāpui as Māori and as a rainbow person, ask about how their identity as Māori interacts with their rainbow identity, and support those looking for (re)connection with te ao Māori.

\section{Pasifika Rainbow People}

Rainbow identities have long been part not only of te ao Māori, but of other Pacific cultures. 'Pasifika' is a term often used for New Zealand-born people of Pacific heritage. Pasifika identities are more complex than a single ethnic identity, as Pasifika peoples typically navigate their cultural heritage identity, their identity as a New Zealander, and a diasporic Pasifika identity (this diasporic identity can diverge from 'traditional' cultures, e.g. Samoan or Tongan, while still embracing elements of those cultures).

\section{Indigenous Rainbow Identities Across the Pacific}

Traditional rainbow identities across the Pacific include Mahu (Hawaii), Vakasalewalewa (Fiji), Palopa (Papua New Guinea), Fa'afafine or Fa'afatama (Samoa, American Samoa), Aka'vaine (Cook Islands), Leiti (Tonga), Fakafifine (Niue) and many more. These often come with particular social roles and responsibilities. Because Western concepts don't always apply within other cultural contexts, each term is best understood within its own cultural context.

\section{Pasifika Rainbow Identities and Colonisation}

Although rainbow identities have always been part of Pacific cultures, colonisation disrupted traditional understandings of sexuality and gender. Because of this, Pasifika rainbow people may face silence and avoidance of their identities within families, churches, and wider communities. Pasifika rainbow people might also feel pressure to navigate their gender and sexual identity according to Western norms which are not compatible with their wider communities or family beliefs.

\section{Accessing Mental Health Support}

When supporting Pasifika rainbow people, check in about what terms and pronouns are right for them. Some Pasifika people will use indigenous terms like those noted above, while others might connect with terms like 'gay', 'bisexual', and 'transgender', or use different terms in different contexts. Similar to supporting takatāpui, ask about how clients' identity as Pasifika intersects with their rainbow identity, and take time to learn about Pasifika identities and cultural worldviews. 


\section{Multiple Gender Attracted People}

Multiple gender attraction is an umbrella term for attraction to people of more than one gender. The most common sexual orientations under this umbrella are bisexuality and pansexuality.

\section{What's the Difference Between Bisexuality and Pansexuality?}

- Some people define bisexuality as attraction to both men and women, and others define bisexuality as attraction to people of more than one gender.

- Pansexuality is typically defined as attraction to people regardless of their gender. For many people, use of the term 'pansexual' explicitly acknowledges that there are more than two genders.

Depending on which definitions someone uses, bisexuality and pansexuality can be interchangeable terms or can be very different to one another. Some multiple gender attracted people use both terms to describe themselves, others use one or the other, and others use a different term (e.g. polysexual).

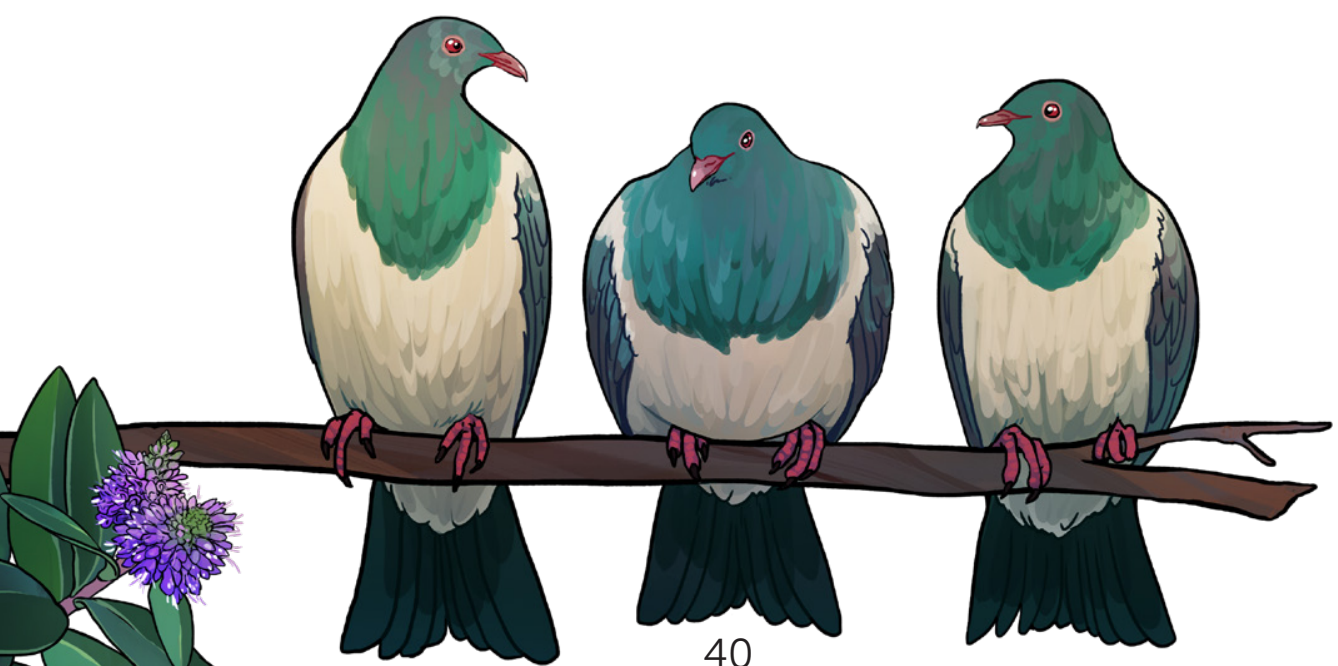

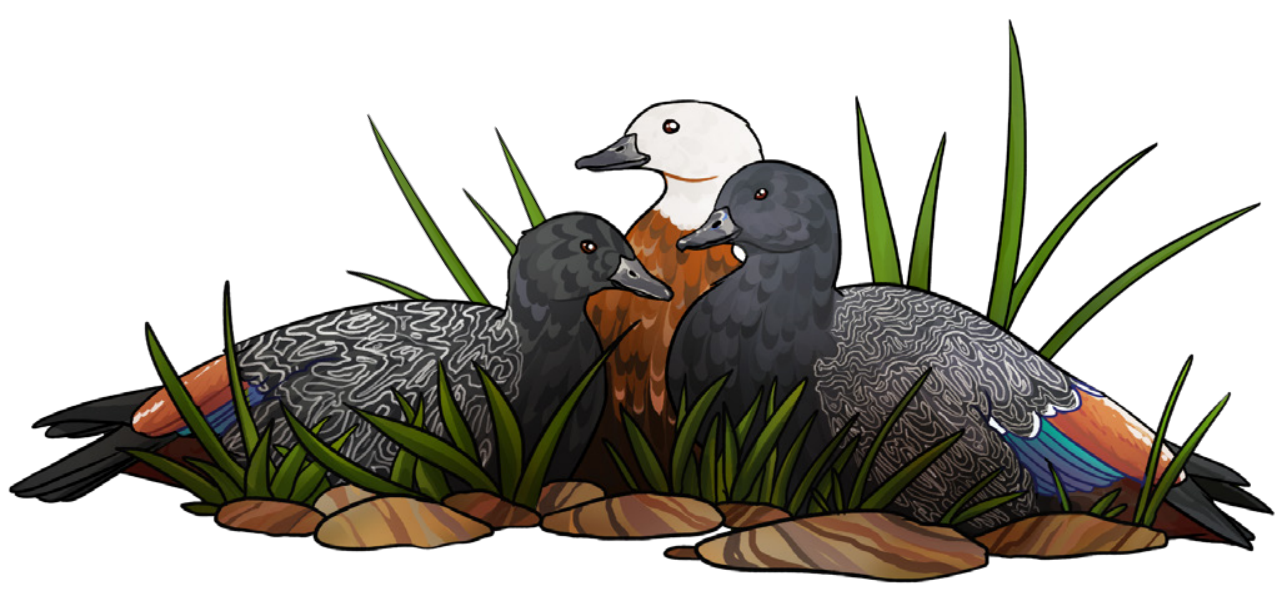

Stereotypes and Misconceptions About Multiple Gender Attraction

Sexual orientation is often misunderstood as binary. Because of this, multiple gender attraction is sometimes viewed as a phase, with others assuming that multiple gender attracted people will eventually be gay or straight but haven't yet 'made up their mind'.

Other common stereotypes are that multiple gender attracted people are seeking attention, promiscuous, or need to feel a certain 'amount' of attraction to people of different genders to truly be attracted to multiple genders. For many people attracted to multiple genders, their current relationship is taken to be indicative of their sexual orientation. For example, a woman in a relationship with a woman might be assumed to be lesbian, even though she is also attracted to people of other genders.

\section{Multiple Cender Attraction and Mental Health}

The mental health disparities experienced by rainbow people tend to be exacerbated among multiple gender attracted people. Researchers attribute this to widespread invisibility, biphobia, and non-acceptance from both gay and straight communities. 


\section{Infersex/VSC People}

Intersex or variations in sex characteristics (VSC) are terms used when someone's sex characteristics are more diverse than the typical definitions of male and female. Within medical settings, there is a widespread lack of knowledge about how best to support intersex people or people with variations in sex characteristics.

One of the most harmful misconceptions is that being intersex is something to be 'fixed.' Intersex people (or parents of intersex babies) are still pressured to undergo non-lifesaving medical interventions (e.g. surgeries or hormone therapy) for cosmetic purposes, or in order to make their bodies fit the male/female binary. This sometimes occurs soon after birth, meaning many intersex people have not had the opportunity to consent to medical intervention. Additionally, there is a great deal of silence and secrecy surrounding intersex identities, or having a variation in sex characteristics.

\section{The Role of Mental Health Professionals}

Referral to psychosocial support and peer support is essential for many intersex people, or people with variations in sex characteristics, as well as their family/whānau. Intersex people often need space, time, and an empathetic sounding board to consider questions about who they are, what being intersex means for them, and how it impacts on their relationships with others.

Intersex people might also want information about intersex communities. These questions might come up at the time they found out they're intersex, or many years later. Family/whānau members of intersex people may want support working through their feelings of worry or grief for their whānau member, as well as help advocating for them in the healthcare system.

\section{Asexual People}

Asexual people do not experience sexual attraction, or interest in and desire for sex.

Asexuality is commonly confused with:

- Celibacy (where someone may experience sexual attraction but choose not to have sex for personal or faith-based reasons);

- being aromantic (not experiencing romantic attraction); and

- being agender (not identifying with any gender).

Many asexual people are in romantic relationships and may identify with terms such as homoromantic, biromantic, and heteroromantic. Some asexual people have sex (as sexual arousal can exist without sexual attraction).

\section{The Spectrum Between Asexuality and Sexuality}

Grey asexuality is the grey area between asexuality and sexuality People along this part of the spectrum might experience sexual attraction on occasion or might feel sexual attraction only after developing a close relationship with someone. They might describe themselves as 'demisexual' or 'grey-ace.'

\section{Pathologisation of Asexuality}

Many conditions described in the DSM sound very similar to asexuality (e.g. Male Hypoactive Sexual Desire Disorder and Female Sexual Interest/Arousal Disorder, which include symptoms like 'absent interest in sexual activity'). Because of this, asexuality can be pathologized or misunderstood as a disorder. Asexual people are, in fact, excluded from these diagnoses, but still face misconceptions that everyone wants sex, likes sex or will have sex. Asexual people might want to talk about being made to feel abnormal, or about being bullied or pressured by societal norms around sex and sexuality. 


\section{Non-Monogamous People}

In a non-monogamous relationship, the people in the relationship have agreed that it's okay for one or more of them to have other partners. Non-monogamous relationships are also called polyamorous relationships or open relationships.

There are a few important things to know about non-monogamy:

- Non-monogamy occurs in both rainbow and heterosexual relationships, but unhelpful attitudes towards rainbow and nonmonogamous relationships often overlap (e.g. relationships not being taken seriously). This means that non-monogamous rainbow people are doubly exposed to these unhelpful attitudes.

- Different people use different words or labels to describe their relationships (e.g. non-monogamous, polyamorous, open). It's a good idea to check what the word or label means for your client.

- Non-monogamy is not the same as infidelity. It's a life choice that aims to be ethical, meaning that all partners involved in relationships consent to the arrangement and are treated respectfully.

- There are some misconceptions that non-monogamous people don't feel jealousy, or that if someone feels jealous it means non-monogamy isn't for them. Neither of these things are true people in any kind of relationship can feel insecurity or jealousy and might want to talk through these feelings in therapy.

- Some people see non-monogamous people as a marginalised group, and others don't. Either way, it is something that can bring judgement from others, which non-monogamous people may need support within mental health settings.

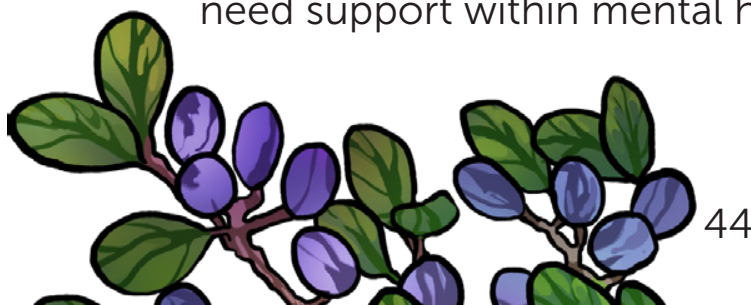

\section{Rainbow People with Disabilities}

There are many types of disabilities, including those that affect a person's vision, movement, thinking, remembering, learning, communicating, hearing, mental health, and social relationships. The social model of disability is based on disability rights and offers an alternative to deficit-based models which think of disabilities as something to be fixed.

For some people identifying as disabled is a key part of who they are, while others may not describe themselves as having a disability. Some people have more than one disability. It is essential to check in about what terms a person uses to describe themselves and to use these same terms. When supporting rainbow people with disabilities, keep these points in mind:

- Not all disabilities are visible, so many rainbow people with disabilities have to come out about their disability, as well as their rainbow identity.

- People with disabilities are often assumed to be disinterested in sex and relationships, or assumed to be straight and cisgender. Because of this, many rainbow people with disabilities don't get the sexuality education they need or are not asked about sex and relationships by health professionals

- Sometimes, healthcare professionals focus on one of their clients' identities at the expense of others. Peoples' sexuality or gender can also be disregarded because of their disability (this is common for people who are both trans and autistic).

- Rainbow groups, events, and spaces may be inaccessible for people with disabilities. On the other hand, disability groups, events, and spaces may not be rainbow friendly. This can limit the supports available to rainbow people with disabilities. 


\section{and Asylum seekers}

Refugees are those who have fled their country because they are at risk of serious human rights violations and persecution. Asylum seekers have fled their country for the same reason and are seeking international protection but have not yet been recognised as refugees (this process can take 10-12 months). Once an asylum seeker has lodged their claim seeking refugee status, they can access the same public healthcare services as other refugees (including gender-affirming health services)

\section{Rainbow Identities and Human Rights}

In many countries, rainbow people are subject to serious human rights abuses. This can include violence from family, community members and government representatives, lack of police protection, severe discrimination, and exclusion from access to basic services and social support. Many rainbow people are forced to leave their home country as a result, in search of safe places to live.

\section{Rainbow Refugees and Asylum Seekers in Aotearoa}

Refugees or those seeking asylum are typically traumatised by the persecution they have experienced. Once here, an asylum seeker has to tell their life story to claim refugee status. It is typically retraumatising, often comes with no counselling support, and can include a need to 'prove' one's sexuality or gender. This creates a catch-22 for those who have previously had to hide who they are or may have no equivalent terms in their own language to describe their identity. For many, arrival here does not mean the end of violence and discrimination; they face racism, xenophobia and prejudice. It is common for rainbow refugees and asylum seekers to avoid their local migrant community or to not disclose their identity. As a result, they are often extremely isolated socially.

\section{Accessing Mental Health Support}

When supporting rainbow refugees and asylum seekers, direct them to refugee lawyers as soon as possible for any visa or asylum issues and establish links with rainbow-friendly and knowledgeable interpreters. Take time to understand clients' specific cultural background and context, seek appropriate supervision, and explore what community organisations they would feel safe approaching for support.

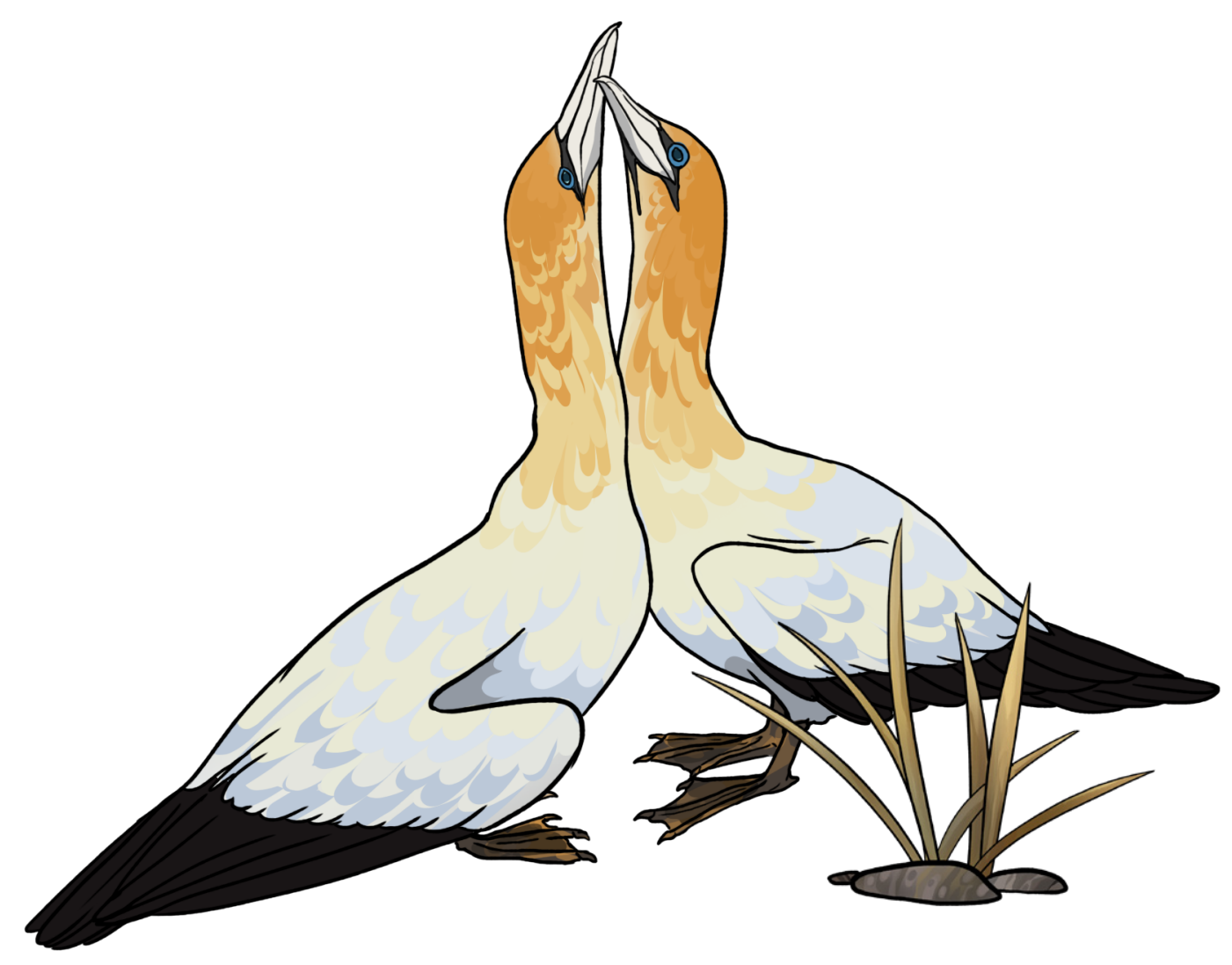


Thank you for supporting our kaupapa by reading this guide. We hope you feel more confident supporting Aotearoa's rainbow people.

We plan to update this guide in future years to reflect changes in knowledge and practice. If you have feedback or questions about this resource, head to our website:

\section{www.rainbowmentalhealth.nz}

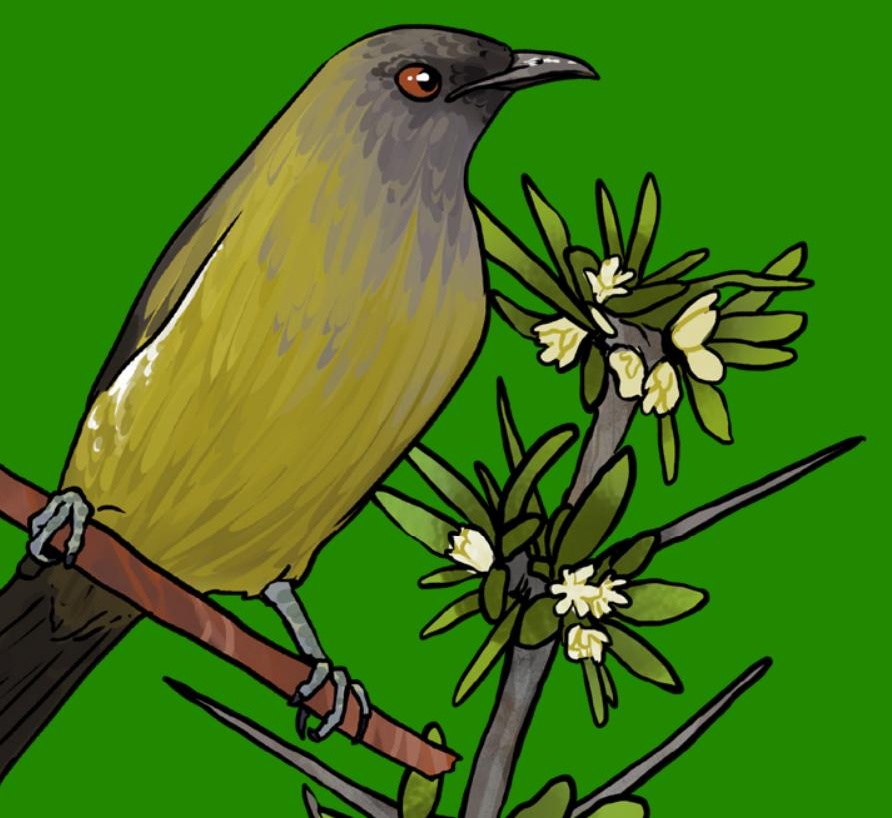

\section{Further Reading}

There was a lot more information we wanted to include in this resource, but couldn't due to space and resource constraints. We encourage you to further your knowledge with the following research and resources.

\section{Rainbow refugees and asylum seekers}

British Psychological Society. (2018). Guidelines for psychologists working with refugees and asylum seekers in the UK: Extended version. http://www.infocoponline.es/pdf/GuideRefugees.pdf

\section{Sex work}

Global Network of Sex Work Projects. (2018). Briefing Paper: The Homophobia and Transphobia Experienced by LGBT Sex Workers. https://www.nswp.org/sites/nswp.org/files/bp_ homophobia_transphobia_mpact_nswp_-_2018.pdf

\section{Alcohol and other drugs}

Rainbow Health Ontario. (2015). LGBTQ People, Drug Use and Harm Reduction. https://www.rainbowhealthontario. $\mathrm{ca} /$ resources/rho-fact-sheet-lgbt2sq-people-drug-use-harmreduction/

\section{Supporting older people}

Addis, S., Davies, M., Greene, G., MacBride-Stewart, S., \& Shepherd, M. (2009). The health, social care and housing needs of lesbian, gay, bisexual and transgender older people: a review of the literature. Health \& social care in the community, 17(6), 647-658. https://onlinelibrary.wiley.com/doi/full/10.1111/ j.1365-2524.2009.00866.x

\section{Sexual and partner violence}

Dickson, S. (2016). Hohou Te Rongo Kahukura: Outing Violence. www.kahukura.co.nz/wp-content/uploads/2017/07/ Building-Rainbow-Communities-Free-of-Partner-and-SexualViolence-2016.pdf 


\section{References}

\section{Language and terminology}

Gender Minorities Aotearoa. (2017). Glossary of Gender Related Terms and How to Use Them. www.genderminorities.com/database/glossary-transgender

Intersex Awareness New Zealand. (2013). What is Intersex? www.ianz.org.nz/ what-is-intersex

RainbowYOUTH. (2019). Info and Useful Links. ry.org.nz/what-we-do/infouseful-links

\section{Key concepts}

Crenshaw, K. (1993). Mapping the margins: Intersectionality, identity politics, and violence against women of color. Stanford Law Review, 43, 1241-1299.

Meyer, I. H. (1995). Minority stress and mental health in gay men. Journal of Health and Social Behavior, 36(1), 38-56.

\section{Rainbow experiences of accessing mental health support in Aotearoa}

Fraser, G. (2019). The Rainbow Mental Health Support Experiences Study. https://10.31219/osf.io/cwzjr and https://10.31219/osf.io/n7apy

RainbowYOUTH \& We Are Beneficiaries. (2018). Out Loud Aotearoa. https://www.facebook.com/OutLoudAotearoa

\section{Needs and experiences of different rainbow groups}

Asexuality New Zealand Trust. (2019). What is Asexuality? www.asexualitytrust.org.nz/what-is-asexuality

Immigration New Zealand. (2019). Refugees, Asylum seekers and their families. immigration.govt.nz/audiences/supporting-refugees-and-asylum-seekers

InsideOUT. (2018). More than Four: A video resource exploring the identities and experiences of and beyond 'LGBT' identities. www.insideout.org.nz/ more-than-four
Kerekere, E. (2015). Takatāpui: Part of the Whanau. Auckland: Tiwhanawhana Trust and Mental Health Foundation. www.takatapui.nz

More Than Two. (n.d.) Polyamory Basics. www.morethantwo.com/polyamorybasics.html

Robertson, S. (2017). All of Us: Minority Identities and Inclusion in Aotearoa New Zealand. https://theallofusproject.net/resource

\section{Gender-affirming healthcare}

Murad, M. H., Elamin, M. B., Garcia, M. Z., Mullan, R. J., Murad, A., Erwin, P. J., \& Montori, V. M. (2010). Hormonal therapy and sex reassignment: A systematic review and meta-analysis of quality of life and psychosocial outcomes. Clinical Endocrinology, 72(2), 214-231.

Oliphant J, et al. (2018). Guidelines for gender affirming health care for gender diverse and transgender children, young people and adults in Aotearoa, New Zealand. www.researchcommons.waikato.ac.nz/handle/10289/12160

Temple Newhook, J., Pyne, J., Winters, K., Feder, S., Holmes, C., Tosh, J., ... \& Pickett, S. (2018). A critical commentary on follow-up studies and "desistance" theories about transgender and gender-nonconforming children. International Journal of Transgenderism, 19(2), 212-224.

\section{Other cited research}

Ard, K. L., \& Makadon, H. J. (2011). Addressing intimate partner violence in lesbian, gay, bisexual, and transgender patients. Journal of General Internal Medicine, 26(8), 930-933.

Dodge, B., Sandfort, T. G., \& Firestein, B. (2007). A review of mental health research on bisexual individuals when compared to homosexual and heterosexual individuals. In B. A. Firestein (Ed.), Becoming visible: Counseling bisexuals across the lifespan (pp. 28-51). New York: Columbia University Press.

Fedewa, A. L., Black, W. W., \& Ahn, S. (2015). Children and adolescents with same-gender parents: A meta-analytic approach in assessing outcomes. Journal of GLBT Family Studies, 11(1), 1-34. 


\section{Rainbow Organisations in Aotearoa}

\section{Gender Minorities Aotearoa}

A cross-cultural, transgender led organisation which aims to facilitate health and well-being for takatāpui, transgender, and intersex populations.

www.genderminorities.com

\section{InsideOUT}

InsideOUT provides workshops, resources and support to help make schools, community organisations and workplaces inclusive for rainbow people.

www.insideout.org.nz

\section{Intersex Awareness New Zealand}

Intersex Awareness New Zealand provides information, education and training for organisations and professionals who provide services to intersex people and their families.

www.ianz.org.nz

\section{OUTLine}

A confidential support line for people in the rainbow community. www.outline.org.nz

\section{RainbowYOUTH}

RainbowYOUTH is dedicated to helping young queer and gender diverse (LGBTIQ) people up to the ages of 27, as well as their wider communities.

www.ry.org.nz

There are many more organisations where you can seek support, information, and training. To find rainbow organisations in your area, visit www.imlocal.co.nz for a directory of queer and gender diverse support groups all over Aotearoa.

This resource was created with funding support from:

\section{$\underline{\text { RULE FOUNDATION }}$}

:0908 Rainbow New Zealand

$\because \quad$ Charitable Trust

\section{oakley mental health}

research foundation
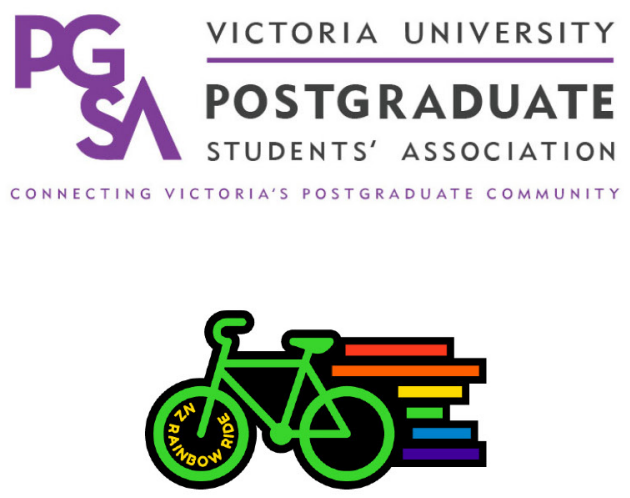


\section{Find out more at rainbowmentalhealth.nz}

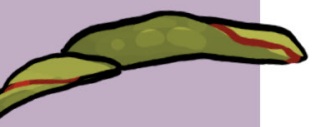

\section{InsideCUT}

\section{RainbowYOUTH}

WELLBEING STUDY
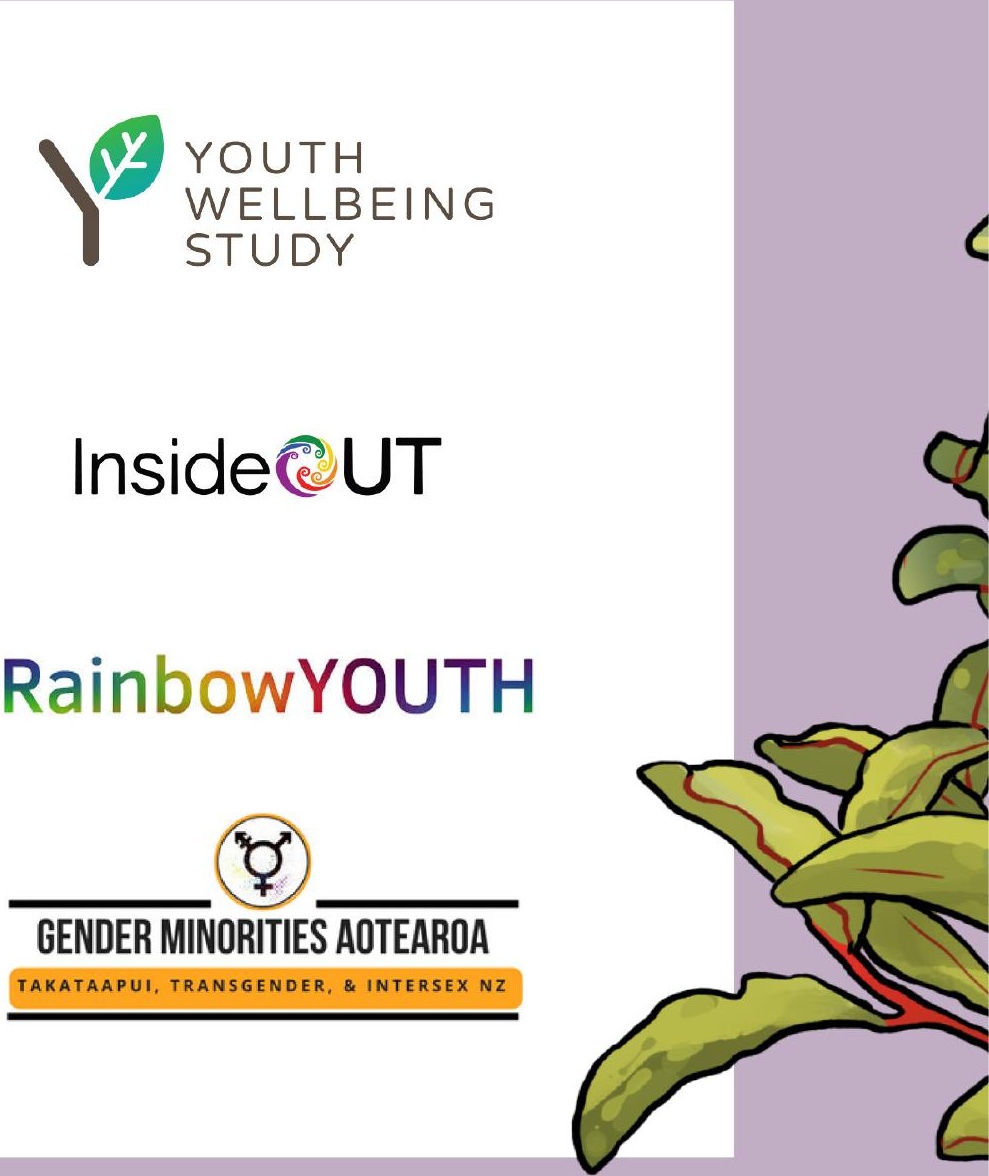

Copyright Youth Wellbeing Study, RainbowYOUTH 2019

ISBN (PDF): 978-0-473-48497-2

ISBN (Softcover): 978-0-473-48496-5

Citation: Fraser, G. (2019). Supporting Aotearoa's rainbow people: A practical guide for mental health professionals. Wellington: Youth Wellbeing Study and RainbowYOUTH 


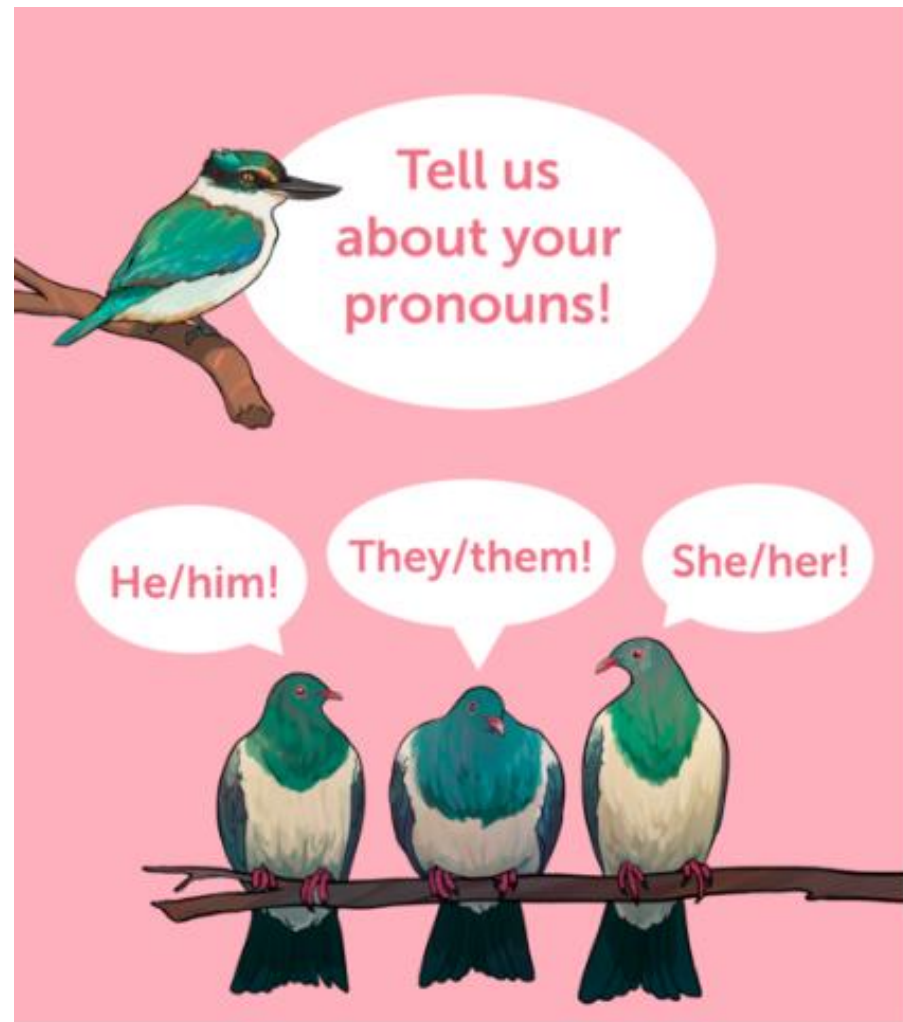

口綵 质 supporting rainbow people, visit

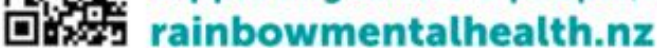

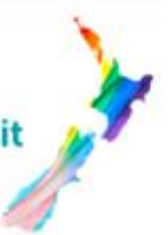

Figure 21. Poster encouraging sharing pronouns

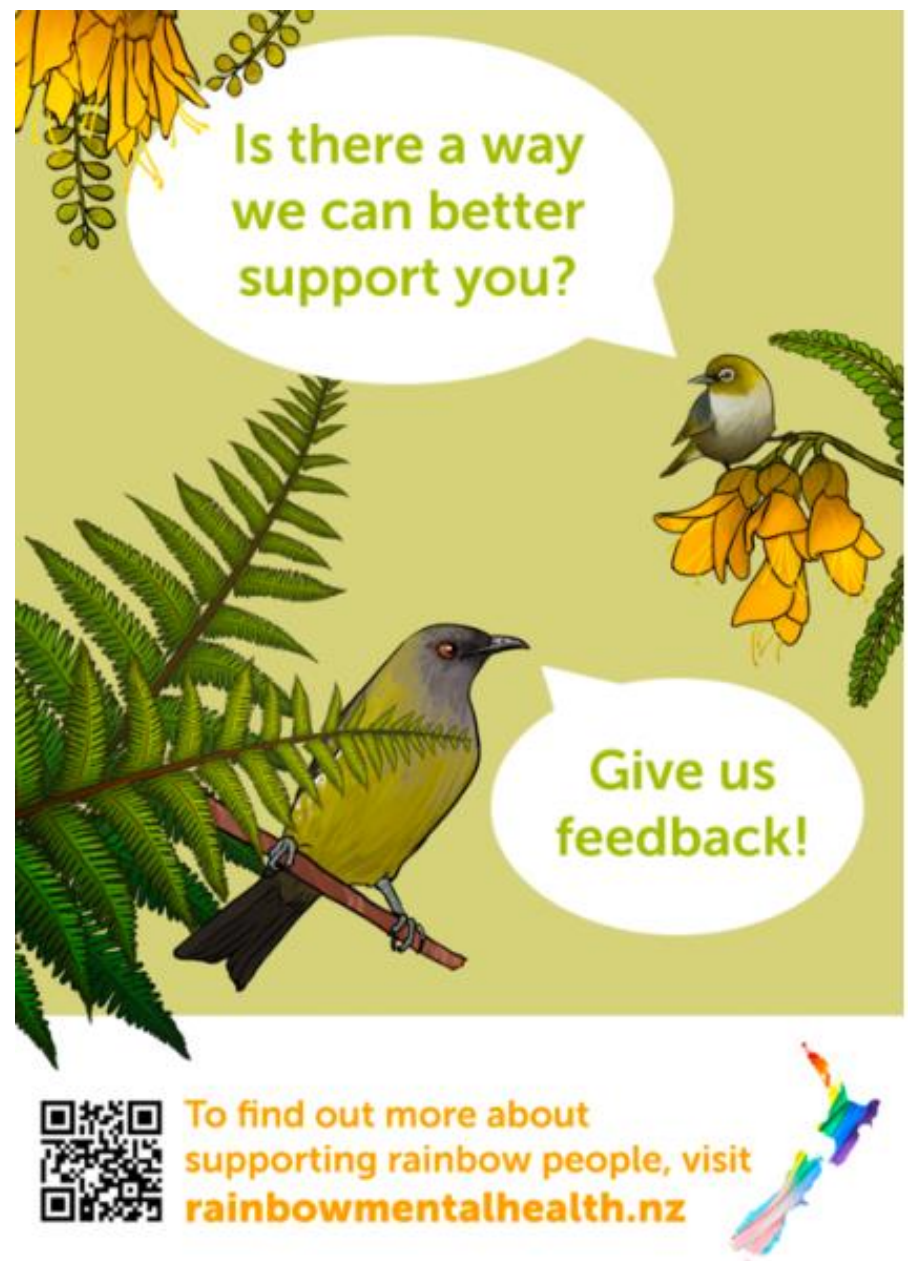

Figure 22. Poster encouraging sharing feedback 

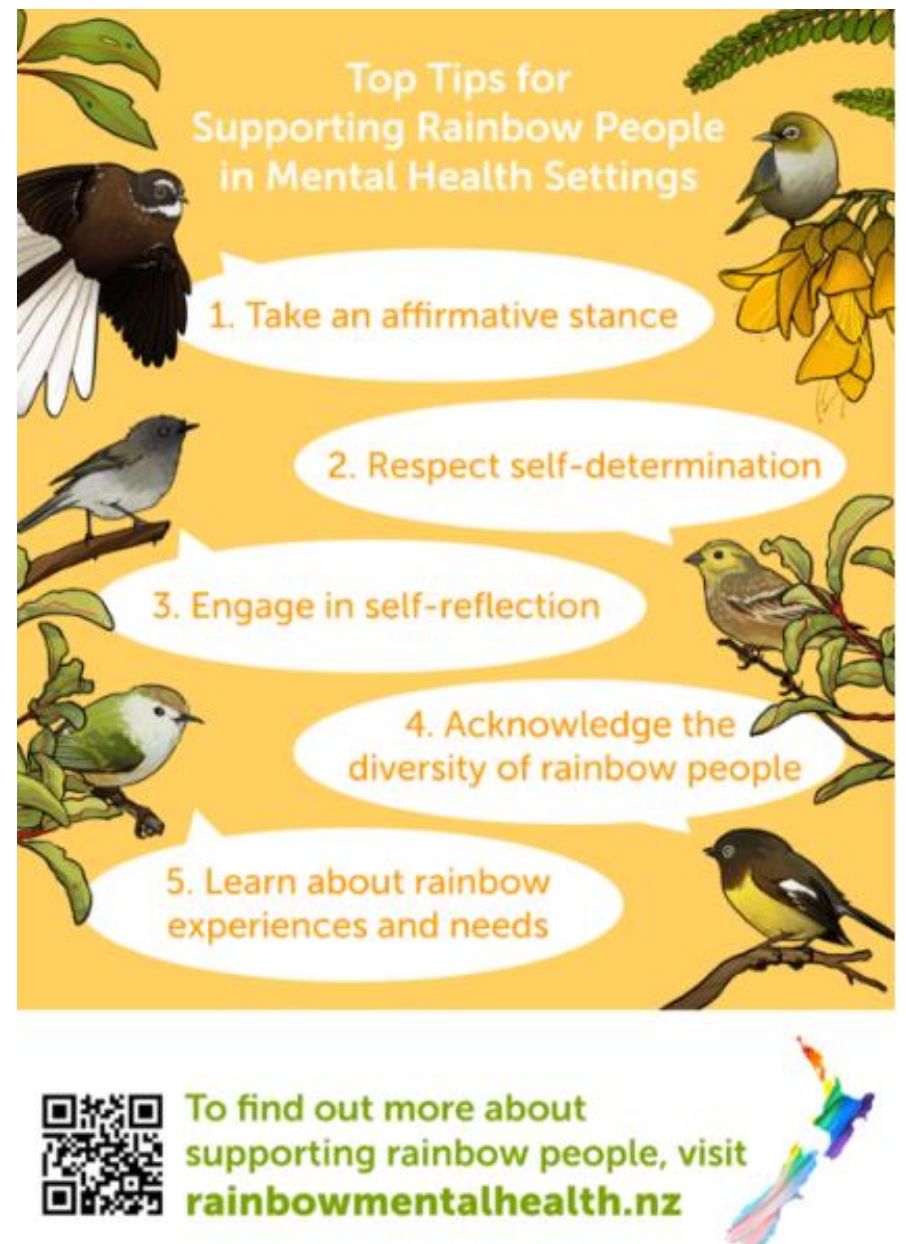

Figure 23. Poster of top tips for mental health professionals.

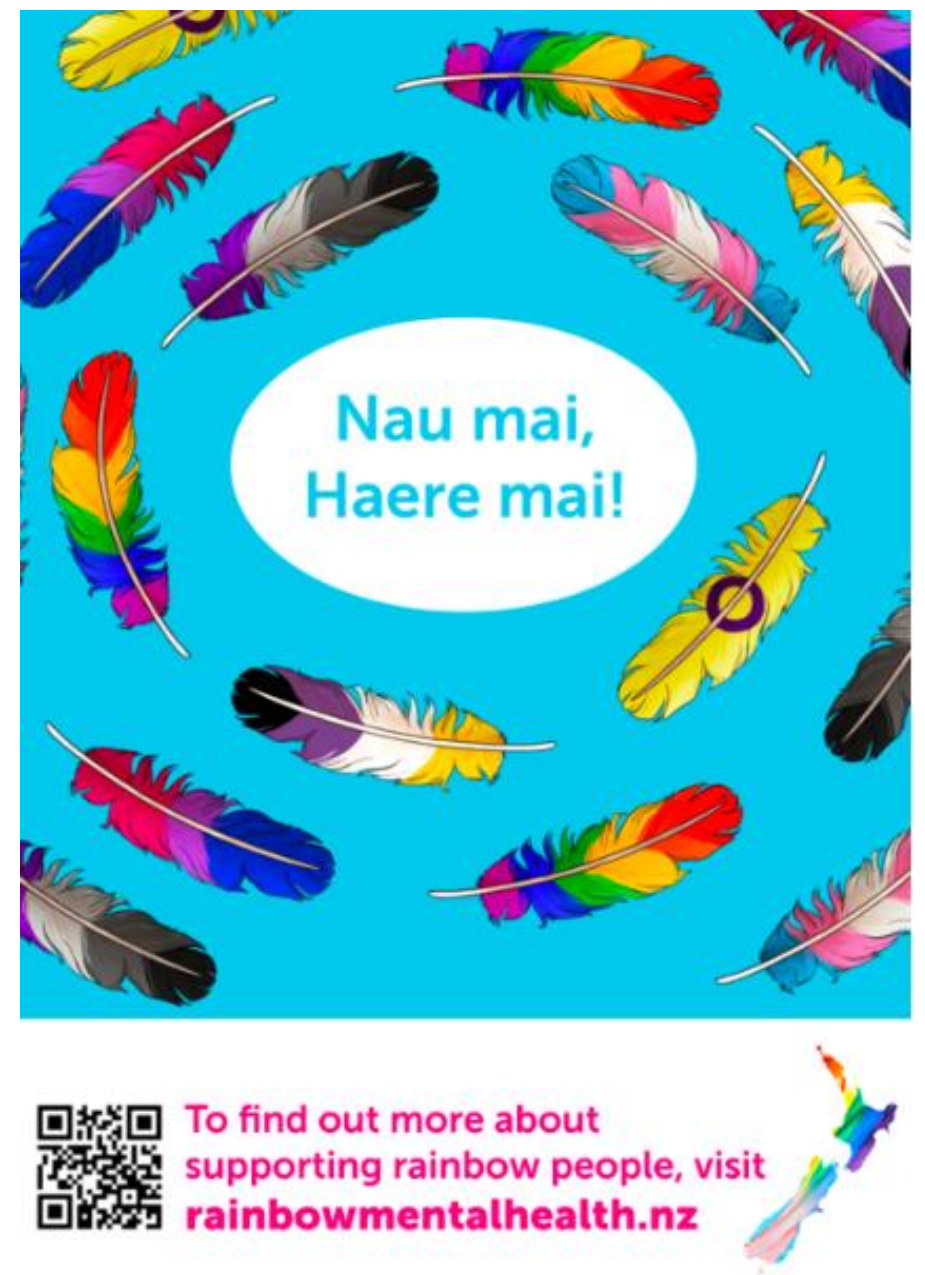

Figure 24. Poster for creating a welcoming space 


\section{Chapter 10: Reflections and Final Thoughts}

In this thesis, I aimed to answer the following research questions:

- What are the experiences of rainbow people who access mental health support in New Zealand?

- What are the experiences of trans people who undergo mental health assessments for gender-affirming healthcare in New Zealand?

- How can these findings inform the provision of high quality and responsive mental health care for queer, trans, and intersex people in New Zealand?

I have addressed my research questions through the lens of queer theory, using both qualitative and quantitative methods, and a reflexive, community-based approach. In this chapter I reflect on my overall findings, situate them within the wider research literature, and discuss their implications and applications. I also outline the strengths and limitations of the research I undertook, in order to inform future work on the mental health care of rainbow people. I shall reflect on the research process, discussing my thoughts about my own positioning in the research, community-based research, and what it was like to carry out this research at this time in New Zealand. Finally, I outline recommendations for future research and policy.

\section{Reflections on Research Findings}

Although the high rates of mental health difficulties in New Zealand's rainbow communities are well documented (Fergusson et al., 1999, 2005; Fraser et al., 2017; Lucassen et al., 2014; Skegg et al., 2003), prior to this project there was a dearth of research about the experiences of rainbow people who have accessed mental health support in Aotearoa (for exceptions, see Birkenhead \& Rands, 2012; Semp, 2006; Welch et al., 2000). This thesis has provided valuable insight into the therapy-related experiences of New Zealand's rainbow people, and how it might be improved. My results suggest that the quality of mental health support varies widely for rainbow people in New Zealand. Some found their mental health professionals affirming, 
validating, helpful and supportive. For many rainbow people, however, their experiences in mental health settings left plenty of room for improvement.

This overall finding that rainbow people have mixed experiences of accessing mental health support is reflected in previous New Zealand (Adams et al., 2012; Birkenhead \& Rands, 2012; Semp, 2006; Welch et al., 2000) and international (Filice \& Meyer, 2018; King et al., 2007; White \& Fontenot, 2019) research. When participants in the current study described negative experiences in mental health settings these tended to reflect therapist microaggressions and assumptions (e.g. a surprised look when the participant came out) and a lack of knowledge on the part of mental health professionals, rather than instances of overt discrimination such as verbal harassment or encouraging clients to 'renounce' their sexual orientation. This is consistent with an overall trend in the literature, where more recent studies of rainbow therapy experiences (e.g. Eady et al., 2011; Hunt et al., 2006; McCullough et al., 2017; Poteat et al., 2013; Shelton \& Delgado-Romero, 2011) tend to report more subtle forms of discrimination than the descriptions of overt prejudice in older studies (e.g. Golding, 1997; Lucksted, 2004; Nystrom, 1997). Changes in the nature of the therapy experience could indicate a general trend towards rainbow-affirmative practice among mental health professionals, alongside societal shifts in the cultural and legislative inclusion of rainbow people (e.g. Marriage Equality in the US, Canada, and New Zealand).

Across the interviews and surveys participants reported long waiting times to access support, and many had their referrals rejected due to not meeting the cut-off for publicly funded mental health support (moderate to severe mental illness or psychological distress; Healthpoint, 2019). Those who did receive support were often given a limited number of sessions, which did not leave adequate time for them to address or work through all their difficulties. Although there is little research about structural barriers to accessing mental health support in the New Zealand context, participants' experiences closely mirrored media reports about the treatment gap in Aotearoa's mental health services (Hutton, 2017; McAllen, 2017; Meier, 2017; Wright, 2016), a recent needs assessment on the mental health promotion and suicide prevention needs of rainbow New Zealanders (Adams et al., 2012) and were also consistent with findings of the People's Mental Health Report (Elliott, 2017) and the 2018 Government Inquiry into Mental Health and Addictions services (which was published shortly after I 
gathered my data). It is likely that difficulties accessing care are exacerbated among queer, trans, and intersex people. Participants noted that it is important that services are rainbow-friendly, however very few services advertise themselves as such, which may restrict the number of services that they feel safe to access. The small number of explicitly rainbow-friendly services also means that services that $d o$ advertise as rainbow-friendly are often overwhelmed by demand (Kondou, 2017).

Participants in the current study generally saw services as embedded within a heteronormative and cisnormative societal context, rather than seeing them as a safe place outside of these structural systems. This meant that participants were sensitive to the possibility of facing stigma and discrimination in mental health settings, which subsequently impacted on their ability to safely discuss their rainbow identity. Many participants described the way they actively negotiated coming out, and there was a great deal of variation in participants' comfort about coming out when meeting a new mental health professional. This finding is also seen in the wider research literature, with almost a third of participants in McCann and Sharek's (2014) study reporting that they could not discuss their LGBT identity with their mental health provider, and numerous other studies noting that fear of discrimination is a barrier to help-seeking (Daley, 2010; Dobinson \& Macdonnell, 2005; Evans \& Barker, 2010; Hiestand et al., 2007; Hunt et al., 2006; Robertson, 1998; Semp, 2006; Shelton \& Delgado-Romero, 2011; Sperber et al., 2008).

Perhaps the most prominent theme across the interviews and surveys was that participants felt their mental health professionals demonstrated a widespread lack of knowledge about sexuality, gender, and sex characteristic diversity, and about rainbow peoples' needs and experiences. Because of this, participants often felt the need to educate their mental health professional, either to move forward in therapy, or for the sake of the rainbow clients that would follow them. This need to educate mental health professionals is reported throughout the wider literature (Bauer et al., 2009; Corliss et al., 2007; Evans \& Barker, 2010; Grove, 2009; Kidd et al., 2011; Lucksted, 2004; McCann \& Sharek, 2014; McIntyre et al., 2011). As in other studies (Eady et al., 2011; Evans \& Barker, 2010; Israel et al., 2008; Page, 2004) participants commonly talked about the importance of basic skills of active listening and validation, noting that their mental health professionals had not allowed the client to direct the focus of therapy. 
Instead many mental health professionals had either focussed on their clients' identity when it was not the issue or refused to discuss this when the client said that it was relevant.

In both the interview and survey study, almost three quarters of participants who had accessed gender-affirming healthcare had a mental health assessment as part of this process. When describing gender-affirming healthcare experiences participants reported significant structural barriers to accessing care, and also shared that mental health professionals often acted as gatekeepers in this process, rather than as a source of support or information. Gender-affirming healthcare was described as a "postcode lottery", meaning access to care is largely determined by where in Aotearoa someone lives, and many participants felt a need to prove their gender, and tell a particular trans narrative in order to access care. This closely reflects international research in which trans people describe their mental health professionals as gatekeepers (Lev, 2004; Sperber et al., 2008; Taylor, 2013), and feel a need to conform to a dominant narrative (Ellis et al., 2015; McNeil et al., 2012; Violeta \& Langer, 2017). Findings regarding structural barriers were consistent with results from Counting Ourselves (Veale et al., 2019), the first survey of health and wellbeing of trans and nonbinary people in Aotearoa New Zealand, where participants commonly reported that they had not accessed gender-affirming care because they did not know where to go, could not afford to go, or because particular services are not available in New Zealand. My results extend on those of Counting Ourselves, which examined existing use and unmet need for gender-affirming care, but did not explore experiences of mental health assessments for gender-affirming healthcare.

Although my research findings centre around participants' negative experiences in mental health settings, over half of participants had found mental health professionals mostly or extremely helpful. This is important to note, not only because it shows that Aotearoa's mental health professionals have the skills to support rainbow people, but because good practice provides models for how to improve. As in other studies (Burckell \& Goldfried, 2006; Israel et al., 2008; Page, 2004; Rachlin, 2002; Ross et al., 2016; Saulnier \& Wheeler, 2000; Scherrer, 2013), participants described positive relationships with mental health professionals who were knowledgeable about sexuality, gender, and sex characteristic diversity, encouraged clients to determine the focus of 
therapy, and asked respectful, up front questions. Participants did not expect perfection from their mental health professionals; rather, they wanted them to take time to educate themselves outside of their sessions, to own up to their mistakes, and to affirm and validate clients' rainbow identities.

\section{Implications}

As I have noted at several points throughout this thesis, these findings highlight the importance of increased funding for New Zealand's public mental health services and for ensuring that services are available irrespective of where in the country a person lives. The structural barriers to accessing support exacerbates stress for an already highly stressed group (Clunie et al., 2018; Out Loud Aotearoa, 2018), and can lead to rainbow people relying on others in their community for support, shifting the responsibility for providing care from public mental health services to community organisations. Increased funding for public mental health services would hopefully decrease waiting times for services, ensure that more people in need are able to access services, and would mean that clients have access to an increased number of therapy sessions, giving them the time and space needed to address their difficulties. At the time of writing the Government has already made steps towards increasing access to mental health support, with a $\$ 455.1$ million commitment to fund a new universal frontline mental health service (Bennett, 2019). It remains unclear, however, who will staff these new services, given a skills shortage in the mental health sector (Deguara, 2019) resulting from understaffing, low pay and a lack of professional support (Martin, 2019).

My findings also demonstrate a clear need for mental health professionals in New Zealand to increase their rainbow cultural competence by filling gaps in knowledge about sexuality, gender, and sex characteristic diversity. The need to increase rainbow cultural competence is consistent with the New Zealand Psychologists' Code of Ethics (in which Principle Four is 'social justice and responsibility to society; New Zealand Psychologists Board, 2002). The fact that we ran out of hard copies of our resource for mental health professionals within just a few weeks of our launch indicates that mental health professionals are eager to upskill in the area of rainbow cultural competence. Currently, however, rainbow cultural competence is built into very few of New Zealand's mental health training programmes, and 
professional development trainings for mental health professionals are few and far between. When training is provided, it is typically presented as an 'add-on' to core competence training, raising questions as to whether (and how) information about rainbow needs and experiences can be woven into training on mental health core competencies. Gamble Blakey and Treharne's (2019a, 2019b) recent work on overcoming barriers to trans healthcare education in Aotearoa provides insight into how New Zealand could lead the way in rainbow cultural competency training. Specifically, they emphasise the importance of cultivating teachers' and trainee healthcare practitioners' values, "which can significantly influence engagement in learning for either teacher-learner or student" (Gamble Blakey \& Treharne, 2019b, p. 364).

The high levels of discomfort and worry about meeting new mental health professionals, and the fact that this seems to be informed by past therapy experiences, illustrates that clients do not come into mental health settings as blank slates - rather, they bring their history and expectations with them. These findings emphasise that mental health professionals cannot take their clients' trust for granted; this is something to be earned. These findings also highlight that when mental health professionals maintain a silence around rainbow identities, this can send the message to clients that they do not want to discuss sexuality, gender, or sex characteristic diversity. Interviewees suggested a number of ways that mental health professionals can break the silence around rainbow identities and experiences and communicate that they are a safe person to talk to. Directly asking about rainbow identities is one approach (see Barbara \& Doctor, 2007; Semp, 2006), however this could be confronting for some rainbow people, who may feel they are being asked to come out before they are ready. As such, mental health professionals can engage in a number of more subtle actions that signal safety; using expansive language, sharing and checking pronouns, displaying visual signs of support, and promoting institutional inclusivity such as all-gender bathrooms and asking about gender inclusively on forms.

While many of the steps that mental health professionals can take to show they are rainbow-friendly are easy and simple (e.g. displaying a rainbow flag or poster), my findings also call attention to the complexities that come with supporting rainbow people in mental health settings. My overall results demonstrate that responsive therapy for rainbow clients consists of several delicate balancing acts: mental health 
professionals must have knowledge about sexuality, gender, and sex characteristic diversity, but avoid using their knowledge to make assumptions about their client and their needs. Instead, mental health professionals must ensure that they take the time to get to know the person in front of them. ${ }^{79}$ Mental health professionals must also be able to apply their skills flexibly to account for the diversity of rainbow clients. As discussed in Chapter 6, the ways in which validation and affirmation (core features of rainbowaffirmative therapy; Pixton, 2003) is best expressed is likely to differ between different clients, and mental health professionals must attend to subtle cues to guide their therapeutic practice.

\section{Applications}

My research findings and resource could be used to guide the development of rainbow cultural competence training for mental health professionals in New Zealand. Currently, much of this training is carried out by rainbow community organisations, who have a great deal of knowledge about (and lived experience of) the mental health needs of rainbow people but may not have the therapy experience to address questions about clinical practice, psychological formulation, or building the therapeutic relationship. This research provides guidance for mental health professionals attempting to understand the relationship between rainbow identities and mental health, and suggestions for how to approach these conversations in a therapeutic setting.

\section{Strengths of the Research}

There are several strengths to this research, including its large sample size, integration of multiple methods, and exploration of experiences that were previously absent from the extant literature (e.g. experiences of trans people in Aotearoa who have accessed mental health support for gender-affirming healthcare). My online survey was the second largest of any previous study with rainbow people in Aotearoa. This allowed me to examine group differences in rainbow peoples' mental health support experiences

\footnotetext{
${ }^{79}$ I imagine that it would be easy to stray too far in either direction: if a mental health professional were to hold back about what they know their client may feel a need to educate them (which takes up valuable time and can disrupt the therapeutic relationship), however if a mental health professional is too expressive about their knowledge their client may feel that the professional has jumped to conclusions about their experiences on the basis of this prior knowledge.
} 
that had not been explored in past research, and to consider how the cultural specificities of New Zealand's mental health system informed rainbow peoples' experiences of seeking support. By using multiple methods, I was able to draw on the strengths of both qualitative and quantitative approaches, and integrating these methods made my findings more robust. With my interviews I was able to gain an in-depth understanding of what it was like to access mental health support as a rainbow person in Aotearoa, and with my online survey I was able to demonstrate that these experiences are widely shared, which was essential for developing my resource for mental health professionals.

The community-based nature of this research is perhaps its biggest strength. As I will discuss in the next section, conducting community-based research is not always easy, but here it produced research that went beyond answering the questions I proposed at the beginning of the project; it was also of benefit to the communities I was working in. Rainbow community organisations have used my research and resource in their applications for funding, rainbow cultural competence trainings, and to promote the work they do for rainbow people. Many rainbow people have reached out over the course of this project and shared how much it meant to them that a researcher (particularly a straight and cis researcher) cares enough to do this work, and others have shared that this project has challenged their understandings of researchers, and what research can be. The community-based nature of the research also enhanced the quality of this work. The guidance and advice from rainbow community advisors ensured the relevance of my questions, helped me to reach far more participants than I otherwise would have, and was incredibly helpful in my data analysis and interpretations. My resource was also much improved by the feedback from so many mental health professionals and rainbow community members.

\section{Limitations of the Research}

Like any piece of research, this project has several limitations. One of my most significant challenges throughout the research process was attempting to balance breadth and depth. For me, breadth refers to the range of experiences I explore, while depth refers to the level at which I can explore those experiences. As Patton (1990) notes, researchers must always make a trade-off when pursuing breadth and depth; more 
of one generally means less of the other. When conducting this research I often felt that I was aiming for breadth at the expense of depth by interviewing and surveying so many people, and by choosing to incorporate resource development into my already short timeframe. I made this choice as I felt that, with so little existing research, it was important to produce as much knowledge in this area as possible. I also aimed to produce this knowledge in a way that would enable it to be easily disseminated and used widely in practice.

Looking back now I do not think I would structure my project differently if given the opportunity, but I do think the broad scope of this thesis may have compromised the extent to which I was able to consider the nuances of participants' experiences. With more time, I would have aimed to refine my qualitative analysis of interview data and attempted to develop a survey with more targeted measures, particularly around the time at which participants had various experiences in mental health settings, and how they perceived the impact of those experiences. Although I am proud of the community-based nature of this work, this was also limited by my time constraints. If I had an additional six months to work on my resource development, I would have consulted with Māori in the rainbow community so that they could guide the te reo translation of the resource. With this consultation, we may have been able to publish a glossary of rainbow terms in te reo Māori, rather than presenting the te reo version of the resource as "the start of a wider conversation" (see Chapter 9).

Another notable limitation of the project was the limited representation of minority groups within rainbow communities. Throughout the wider literature on rainbow mental health and rainbow peoples' mental health support experiences there is a notable absence of the voices of ethnic minorities, migrants and refugees, older adults, people with disabilities and people living in isolated or rural areas (for exceptions, see McCann et al., 2016; Reading \& Rubin, 2011; Wynn \& West-Olatunji, 2009). Unfortunately, my research was no different. This is likely because minorities within the rainbow community are the hardest to reach of an already 'hard-to-reach' population (Ellard-Gray, Jeffrey, Choubak, \& Crann, 2015). Although I endeavoured to recruit people from these groups, and to consider their needs during my resource development, the lack of engagement with these groups within the wider rainbow community likely means that my findings and resource predominantly reflect the experiences and needs of 
younger, able-bodied Māori and Pākehā, born in New Zealand, and living in New Zealand's larger cities. The fact that I struggled to recruit minority groups within rainbow communities despite my engagement with community organisations and leaders highlights potential challenges not only for researchers, but for advocacy and support organisations - it is likely that these organisations also encounter difficulties reaching the hard-to-reach.

\section{Reflections on Research Process}

I have come across many adages relevant to the $\mathrm{PhD}$ process over the course of conducting this research: it is impossible to say how many times my primary supervisor told me, kindly but firmly, "It's a PhD, Gloria, not a Nobel Prize" ${ }^{80}$ Many whakatauki apply here too - "Tē tōia, tē haumatia", 81 "He pai ake te iti i te kore", ${ }^{82}$ and "E tata tapahi, e roa whakatū." 83 The saying I resonated with the most, though, was that writing a thesis is about the journey, not the destination; the learning I took from the $\mathrm{PhD}$ process was just as important as what I learned from the data itself. Here I reflect on key parts of this journey, including how my feelings about my positioning shifted and changed over the course of the research, the challenges of doing community-based research, and the ways in which my research was informed by my wider cultural context.

\section{Positioning}

My positioning in this research has far and away been my biggest source of anxiety and stress over the course of this project. As this project ends, it is interesting to reflect on the ways in which these worries have shifted and changed over time. At the start of my PhD I was incredibly concerned that I would be unable to gain the trust of the rainbow community and would struggle to find participants to take part in my

\footnotetext{
${ }^{80}$ I once Googled this phrase in a period of procrastination and found an interesting paper by Mullins and Kiley (2002) about the PhD thesis examination process. Apparently experienced examiners make judgements about the quality of a thesis by the time they have read the first two chapters, but I hope I have made the remaining eight an interesting read too.

${ }^{81}$ Nothing can be achieved without a plan, workforce and way of doing things.

${ }^{82}$ A little is better than none.

${ }^{83}$ Procrastination is the thief of time.
} 
research. As I built relationships with community groups and organisations I became anxious about losing their trust, fretting over my conversations about rainbow-related issues (Was I saying the right thing? Should I speak out on rainbow issues? If I do, am I centring myself in the conversation? If I don't, does my silence make me complicit?). Looking back now I realise that the community members I was working with probably did not spend that much time thinking about what I was doing, but at the time these new relationships felt delicate, and I felt constantly uncertain about whether I should even be doing this work.

As my relationships in rainbow communities became more solid and I began to feel like I was trusted, many of these worries fell away. This is not to say concern about my positioning disappeared - just that it changed form. Now that I am considered (at least by some) an expert in this area, I am routinely invited to give talks and training about how mental health professionals can best support rainbow people. Although I am happy to discuss the findings of my research, providing training by myself feels like an overstep - it seems inappropriate to talk about what it feels like to access mental health support as a rainbow person, not having had that experience myself. I have attempted to address this by presenting workshops and trainings in collaboration with rainbow organisations, so I can discuss my research and address questions about therapeutic issues, while the community organisations can share their knowledge of sexuality, gender, and sex characteristic diversity, and talk through their lived experience of stigma and discrimination.

When I have found myself wishing that I could engage in my work without the added pressure of worrying about my positioning I have been encouraged by Pillow's (2003) notion of uncomfortable reflexivity: Pillow (2003) posits that "the qualitative research arena would benefit from more 'messy' examples, examples that may not always be successful, examples that do not seek a comfortable, transcendent end-point but leave us in the uncomfortable realities of doing engaged qualitative research" (p. 193). To me, this means that if I were to ever do this work without some discomfort about my positioning, this would be cause for concern. It should always feel uncomfortable to do work as an outsider, or an insider-outsider (or, perhaps, in any position at all), because this discomfort keeps us alert to potential dangers: speaking on behalf of the people we work with, working on rather than with people, and performing 
allyship for praise and recognition, rather than engaging in research and activism because it is the right thing to do.

\section{Community-based Research}

My experience of conducting community-based research is closely linked to my reflections on positioning. Partly because it aligned with my values, and partly because I was so eager to build trust and demonstrate that I was one of the "good ones", I was very diligent about checking in with community groups and representatives at all stages on the research. As I have already noted, I believe this is a key strength of the research, and ultimately produced a better product than if I had not had such a strong community focus. Using a community-based approach was particularly important given the harm done to rainbow people by mental health practitioners, researchers, and policymakers (see, for example, Drescher, 2008; Pettit \& Hegarty, 2014; Riggs et al., 2019).

Doing community-based work also had its challenges. I soon realised that this kind of work cannot be limited to business hours: it involves volunteering for rainbow organisations when they need it, showing up to marches and protests, and spending time at social events - activities that were, at times, difficult to balance alongside writing a thesis. Another challenge was deciding what to do when community advisors disagreed with each other, which happened routinely. I often felt stuck at these times, as I did not think it was ethical to seek someone's guidance, then choose to ignore it. I navigated this by attempting to compromise when possible, and by being up front about the reasons behind my decisions when I chose not to take someone's advice. If I were to have my time again, I think I might have been clearer when setting up my community relationships by letting them know that while I would do my best to follow all guidance I received, this would not always be possible. I might also choose not to take community members' advice more frequently; as it was, doing so often created a great deal of work, and in many cases the community organisations probably would have courteously accepted my decision not to take on their guidance.

At many points throughout this thesis I struggled with what to call the community aspect of my work. Although I settled on naming it "community-based" work, I often wondered whether I should call it "community-based participatory research" (CBPR; Minkler, 2005) - others have given it this label, and some of my 
community partners refer to it as a "co-design" project. The reason I did not label it CBPR is I ultimately had the final say on all research-related decisions; although I always sought advice from my community partners and gave a great deal of weight to their thoughts when making decisions, they were not equal decision-makers in the research. I also did not share my research funding with my community partners. This has made me reflect on the fact that there is a very high bar for calling research CBPR and made me question just how many self-described "CBPR" projects truly meet this bar. It also made me wonder about the impact of labelling a project "CBPR" when it does not reflect a true partnership and equal decision making - does this mean that community organisations might enter into research partnerships where they are promised equal decision-making power, only to find that they are consulted on the project in a tick box fashion? Do the "CBPR" studies that arguably do not meet CBPR criteria take some of the power away from true CBPR studies? I offer these thoughts not to single out any particular research project, but to emphasise the importance of not overstating the community contribution within community research projects - doing so may put community-based researchers back into a position of untrustworthy 'takers', rather than people who are interested in mutual benefit from research.

\section{The Research Context}

There was a lot happening in relation to rainbow issues in the time I was conducting this research. In 2016, a year before I began conducting the research, Jordan Peterson (a Canadian clinical psychologist and academic) had gained international prominence for claiming that a proposed amendment to the Canadian Human Rights Act, which would add gender identity and expression as prohibited grounds for discrimination, would undermine his free speech (Murphy, 2016). Trump was elected just before I began my $\mathrm{PhD}$ (something that, three years later, I still cannot believe I am writing), and proceeded to systematically attack hard-won LGBT rights in the US (Levin, 2019). Over the course of the project, Trans-Exclusionary Radical Feminists (TERFs) were becoming ever-louder in New Zealand (particularly in Auckland and 
Wellington), which reached a crescendo in response to proposed amendments to the Births, Deaths, Marriages, and Relationships Registration Act. ${ }^{84}$

The Trump election, campaigning of TERFs and prominence of voices such as Peterson's created a societal context which felt more hostile to trans and nonbinary people, and to rainbow people more generally, than it had in a long time. In my view, this was just as much the case in New Zealand as it was overseas, despite having just elected a self-described rainbow-friendly government and having geographical distance from many of the events overseas. This context had a profound impact on my research. There was often a sense of hopelessness among my community collaborators, and they seemed exhausted by having their identities and experiences constantly undermined. There was a raft of other controversies over the course of the research, such as the police involvement in the Auckland Pride march, which was incredibly divisive in the rainbow community (for discussion, see Murphy, 2018).

In many ways, this societal context served as powerful motivation for continuing my work; conducting collaborative research and putting out an affirming resource seemed a powerful way to counteract discriminatory rhetoric. Like many aspects of the work, the societal context also created some difficulties. For example, a few selfidentified detransitioners (see pages 141-143) took part in my research and contacted me with concerns about the gender-affirming healthcare aspects of my thesis. I knew that some of the community organisations I worked with might recommend that I avoid engaging in these conversations, however as a researcher (and future psychologist) I could not disregard their feeling that health professionals had failed them by providing gender-affirming care. This was a lesson for me that I would not be able to align myself with my community partners entirely; though I took an actively trans-affirmative stance, I also thought it important that people who had accessed gender-affirming healthcare and subsequently wish they had not were given the right to speak out about this experience. What they should not be given the right to do, however, is infringe on the ability of others to self-identify their gender and access gender-affirming healthcare.

84 The proposed Bill would make it easier to change one's gender on the New Zealand birth certificate. TERFs expressed concerns that this "risked unintended consequences for women's sex-based rights" (Speak Up For Women, 2019, para. 15). 
It must be noted that, although a lot of difficult things were happening in society over the course of this project, there were some encouraging things happening too. In 2018, Māori psychologist Michelle Levy took Psychology (as an academic discipline and profession) to the Waitangi Tribunal ${ }^{85}$ for failing to adequately address the needs of Māori. This has sparked conversation about how Psychology can improve Māori cultural competence, and to me created a space to bring up the parallel (and intersecting) problem of Psychology failing to meet the needs of the rainbow community. Overall, psychologists (and mental health professionals in general) seemed more open to exploring gaps in their cultural competence and considering how these gaps could be filled. I believe that Levy's work contributed to a context in which mental health professionals were made aware of the need for resources like mine.

\section{What is Next for this Project?}

At the beginning of my thesis, I noted that use of terminology and understandings of sexuality, gender, and sex characteristic diversity shift and change quickly: this thesis captures a particular moment in time, and future work should always be conducted to account for this rapid change. I made a similar comment in my resource, where I stated that we "plan to update this guide in future years to reflect changes in knowledge and practice" (p. 50). I now wonder whether this was a fair claim to make - in reality, I have no idea where I will be after I finish my $\mathrm{PhD}$ and clinical psychology training. Will I be in a position to keep this promise of updating our guide? I have also realised that the people I worked with in rainbow community organisations may also move on, taking knowledge of this project with them. For the foreseeable future, RainbowYOUTH have agreed to maintain the resource website and pass along any queries that I may be able to answer. It is possible that revising the guide could be a future research project that another student could take ownership of - if this sounds like you, get in touch!

\footnotetext{
85 The Waitangi Tribunal inquires into, and makes recommendations on, claims brought by Māori relating to legislation, policies, actions or omissions of the Crown that are alleged to breach the promises made in the Treaty of Waitangi (Waitangi Tribunal, 2019).
} 


\section{Recommendations}

\section{For Policy, Resourcing, and Training}

In this section I put forward my ideal vision of mental health services and training. I am aware that we may be a while away from implementing these recommendations, however I believe that taking steps in this direction will substantially increase the quality of mental health care for rainbow people in New Zealand. So, without further ado, I recommend:

- Funding for mental health services and gender-affirming healthcare services should be significantly increased to allow access to care for all who need it, and access to care should be consistent across all postcodes.

- My thesis resource should be evaluated, and consideration given to distributing the resource to mental health professionals working in New Zealand's public mental health services, non-governmental organisations, private practices, correctional settings, and hospitals. This would not necessarily have to be in hard copy, but could be via email through DHBs, workplaces, and professionals organisations such as the New Zealand Association of Counsellors and New Zealand Psychological Society. ${ }^{86}$

- Rainbow cultural competence training should be incorporated into all mental health training programmes in Aotearoa. This training should go beyond a oneoff workshop or day-long training, but should be woven throughout training on core competencies. For example, questions around gender and sexuality could be incorporated into training on interviewing skills. Questions around experiences of stigma and discrimination (not only based on sexual orientation and gender, but on ethnicity, religion, ability, class, and many other axes of oppression) could be incorporated into training about initial assessments and exploring a client's developmental history. This training should consider the possibility and impact of therapist microaggressions, and involve role plays of

\footnotetext{
${ }^{86}$ I have had varying levels of success to date with encouraging professional associations to share my research and resource. This seems in part to be because such associations are asked to share resources often and do not want to overwhelm their members with communications, but I also get the sense that they may not take this project as seriously as they would if I were not a student.
} 
conversations around coming out, accessing gender-affirming healthcare, facing societal stigma and discrimination, and navigating relationship issues.

- Incorporating rainbow cultural competence into training programmes will reach training mental health professionals, but not practicing mental health professionals. As such, rainbow community organisations should be funded to provide training to practicing mental health professionals. This training could be provided by rainbow people with therapeutic experience, or could be provided in collaboration with knowledgeable mental health professionals outside of the rainbow community. To ensure that mental health professionals attend this training, professional boards may need to make formal training in rainbow cultural competence a requirement for registration or re-registration.

\section{For Future Research}

This project has made a valuable contribution to the literature on rainbow peoples' experiences of accessing mental health support in Aotearoa, however there is a great deal of work still to be done in this area. Future research should address the limitations of the current study, by exploring the experiences of those in the rainbow community that have previously been invisibilised in this research area. This includes rainbow ethnic minorities, older adults, people with disabilities, people living in small towns or rural areas, intersex people, and refugees and asylum seekers. By gaining an understanding of what it means to access mental health support as a rainbow person with these intersecting identities, researchers and community members could create resources for mental health professionals specific to these groups.

I have recommended that rainbow cultural competence training be incorporated into mental health training programmes and offered to practicing mental health professionals. Further research is needed to explore who should be providing this training, in what format it should be delivered, and how to engage students and mental health professionals who may not feel they need such training (either because they already feel competent in their work with rainbow people, or because they do not believe work with rainbow people should differ from work with non-rainbow people). Future work around training could run pilots of training programmes and evaluate these or could work with rainbow community organisations to explore what resourcing they 
would require to develop and run training. Creative solutions are needed to make space in the already full curricula of most mental health training programmes, as well as to explore how mental health professionals could find time to attend rainbow cultural competence training given their heavy workloads and scarce resources for professional development.

Future research could also explore the experiences of mental health professionals who have supported rainbow people in their practice. There is an existing body of international literature exploring the attitudes and experiences of mental health professionals who have worked with rainbow clients (e.g. Alessi, Dillon, \& Kim, 2015; Owen-Pugh \& Baines, 2014), however there is a current dearth of New Zealand research on the topic. Throughout this thesis I have, at times, speculated as to why mental health professionals have done some of the things participants talked about. I have wondered whether sometimes our basic clinical skills escape us when we are faced with a client and have no idea what to do, and whether some of the harmful past literature (e.g. about the supposed connection between sexual orientation and sexual trauma) has been picked up by mental health professionals and used in their practice. I have also described the broader context within which I conducted this research (in which alt-right and transphobic voices are rising in prominence), but have not considered whether, or how, this context might impact on mental health professionals' practice. By conducting research with mental health professionals, researchers and community members could explore some of the barriers to developing rainbow cultural competence in Aotearoa and use the mental health professionals' perspectives to inform future resources and training around rainbow cultural competence.

\section{Final Thoughts}

For this thesis, I had the valuable opportunity to hear from nearly 1600 rainbow people about their experiences of accessing mental health support and gender-affirming healthcare in Aotearoa New Zealand. My research demonstrates that many mental health professionals in Aotearoa provide responsive and affirming care to their rainbow clients. My research also suggests that there is room for improvement in our mental health services, with significant structural barriers delaying (or impeding) access to care, a widespread silence around rainbow identities and needs, and many mental health 
professionals lacking knowledge about sexuality, gender, and sex characteristic diversity. These findings highlight the need for mental health professionals to seek education and training to improve their rainbow cultural competence. By taking active steps to make our therapeutic spaces rainbow-friendly, and by approaching conversations about sexuality, gender, and sex characteristic diversity, I believe that mental health professionals can provide an affirming and validating space to promote the wellbeing of their rainbow clients.

I feel so honoured that participants in this research trusted me with their stories, and I hope that I have done them, and their wider rainbow communities, justice in this thesis. I conclude this research feeling hopeful for the future of rainbow mental health service provision, and excited to see what happens in this space in future. To end with the words of one of survey respondent, when asked what they would tell a room full of training mental health professionals - "You're doing a wonderful job for the most part. Try to approach this with a gentle, open-minded curiosity. You don't have to know all the answers, just be accepting and empathetic." 


\section{References}

Acker, S. (2001). In/out/side: Positioning the researcher in feminist qualitative research. Resources for Feminist Research, 28(3-4), 153-172.

ActionStation. (2019). Campaigns. Retrieved from https://actionstation.org.nz/campaigns

Adams, J., Dickinson, P., \& Asiasiga, L. (2012). Mental health promotion and prevention services to gay, lesbian, bisexual, transgender and intersex populations in New Zealand: Needs assessment report. Auckland, New Zealand: Te Pou o Te Whakaaro Nui, The National Centre of Mental Health Research, Information and Workforce Development.

Adams, J., Dickinson, P., \& Asiasiga, L. (2013a). Mental health issues for lesbian, gay, bisexual, and transgender people: A qualitative study. International Journal of Mental Health Promotion, 15(2), 105-120.

Adams, J., Dickinson, P., \& Asiasiga, L. (2013b). Mental health promotion for gay, lesbian, bisexual, transgender and intersex New Zealanders. Journal of Primary Health Care, 5(2), 105-113.

Aguilar, J. L. (1981). Insider research: An ethnography of a debate. In D. A. Messerschmidt (Ed.), Anthropologists at home in North America (pp. 15-26). Cambridge: Cambridge University Press.

Alcoff, L. (1991). The problem of speaking for others. Cultural Critique, 20, 5-32.

Alessi, E. J., Dillon, F. R., \& Kim, H. M. S. (2015). Determinants of lesbian and gay affirmative practice among heterosexual therapists. Psychotherapy, 52(3), 298307.

Allen, L. (2003). Girls want sex, boys want love: Resisting dominant discourses of (hetero) sexuality. Sexualities, 6(2), 215-236.

Almeida, J., Johnson, R. M., Corliss, H. L., Molnar, B. E., \& Azrael, D. (2009). Emotional distress among LGBT youth: The influence of perceived discrimination based on sexual orientation. Journal of Youth and Adolescence, 38(7), 1001-1014. 
American Psychiatric Association. (1952). Diagnostic and statistical manual of mental disorders (First Edition). Washington, DC: American Psychiatric Association.

American Psychiatric Association. (1968). Diagnostic and statistical manual of mental disorders (Second Edition). Washington, DC: American Psychiatric Association.

American Psychiatric Association. (1980). Diagnostic and statistical manual of mental disorders (Third Edition). Washington, DC: American Psychiatric Association.

American Psychiatric Association. (1994). Diagnostic and statistical manual of mental disorders (Fourth Edition). Washington, DC: American Psychiatric Association.

American Psychiatric Association. (2013). Diagnostic and statistical manual of mental disorders (Fifth Edition). Washington, DC: American Psychiatric Association.

American Psychological Association Task Force on Gender Identity and Gender Variance. (2008). Report of the task force on gender identity and gender variance. Washington, DC: Retrieved from https://www.apa.org/news/press/releases/gendervariant-2.pdf

Anderson, H., \& Goolishian, H. (2009). The client is the expert: A not-knowing approach to therapy. In S. McNamee \& K. J. Gergen (Eds.), Therapy as social construction (pp. 25-39). London: Sage.

Ardern, J. (2018). Inquiry to improve mental health services. Retrieved from https://www.beehive.govt.nz/release/inquiry-improve-mental-health-services

Ardern, J., \& Robertson, G. (2019). Government establishes Rainbow Wellbeing Legacy Fund. Retrieved from https://www.beehive.govt.nz/release/governmentestablishes-rainbow-wellbeing-legacy-fund

Arthur-Worsop, S. (2016, November 14). Mental health help wait times for youth patients “appalling”. NZ Herald. Retrieved from https://www.nzherald.co.nz/health/news/article.cfm?c_id=204\&objectid=1173531 9

Asarnow, J. R., Rozenman, M., Wiblin, J., \& Zeltzer, L. (2015). Integrated medicalbehavioral care compared with usual primary care for child and adolescent 
behavioral health. JAMA Pediatrics, 169(10), 929-937.

Ashton, T. (2005). Recent developments in the funding and organisation of the New Zealand health system. Australia and New Zealand Health Policy, 2, 1-8.

Asia Pacific Forum. (2016). Promoting and protecting human rights in relation to sexual orientation, gender identity and sex characteristics: A manual for national human rights institutions. Sydney: Asia Pacific Forum.

Ault, A., \& Brzuzy, S. (2009). Removing gender identity disorder from the Diagnostic and Statistical Manual of Mental Disorders: A call for action. Social Work, 54(2), 187-189.

Austin, A., \& Goodman, R. (2018). Perceptions of transition-related health and mental health services among transgender adults. Journal of Gay and Lesbian Social Services, 30(1), 17-32.

Austin, S. B., Ziyadeh, N. J., Corliss, H. L., Rosario, M., Wypij, D., Haines, J., ... Field, A. E. (2009). Sexual orientation disparities in purging and binge eating from early to late adolescence. The Journal of Adolescent Health , 45(3), 238-245.

Badham, V. (2018, June 29). Jacinda Ardern is the very hero the global left needs right now. The Guardian. Retrieved from https://www.theguardian.com/commentisfree/2018/jun/29/jacinda-ardern-is-thevery-hero-the-global-left-needs-right-now

Balsam, K. F., Rothblum, E. D., \& Beauchaine, T. P. (2005). Victimization over the life span: A comparison of lesbian, gay, bisexual, and heterosexual siblings. Journal of Consulting and Clinical Psychology, 73(3), 477-487.

Baltieri, D. A., \& De Andrade, A. G. (2009). Schizophrenia modifying the expression of gender identity disorder. Journal of Sexual Medicine, 6(4), 1185-1188.

Barbara, A., \& Doctor, F. (2007). Asking the right questions 2: Talking with clients about sexual orientation and gender identity in mental health, counselling, and addiction settings. Toronto: Centre for Addiction and Mental Health.

Bartlett, A., Smith, G., \& King, M. (2009). The response of mental health professionals 
to clients seeking help to change or redirect same-sex sexual orientation. $B M C$ Psychiatry, 9, 1-8.

Batejan, K. L., Jarvi, S. M., \& Swenson, L. P. (2015). Sexual orientation and nonsuicidal self-injury: A meta-analytic review. Archives of Suicide Research, 19(2), $131-150$.

Bauer, G. R., Hammond, R., Travers, R., Kaay, M., Hohenadel, K. M., \& Boyce, M. (2009). "I don't think this is theoretical; this is our lives": How erasure impacts health care for transgender people. Journal of the Association of Nurses in AIDS Care, 20(5), 348-361.

Baumeister, R. F., Bratslavsky, E., Finkenauer, C., \& Vohs, K. D. (2001). Bad Is stronger than good. Review of General Psychology, 5(4), 323-370.

Baxter, J., Kingi, T. K., Tapsell, R., Durie, M., \& Mcgee, M. A. (2006). Prevalence of mental disorders among Māori in Te Rau Hinengaro: The New Zealand Mental Health Survey. Australian \& New Zealand Journal of Psychiatry, 40(10), 914-923.

Beehler, G. P. (2001). Confronting the culture of medicine: Gay men's experiences with primary care physicians. Journal of the Gay and Lesbian Medical Association, 5(4), 135-141.

Bell, A. P., \& Weinberg, M. S. (1978). Homosexualities: A study of diversity among men and women. New York: Simon and Schuster.

Bene, E. (1965). On the genesis of male homosexuality: An attempt at clarifying the role of the parents. The British Journal of Psychiatry, 111(478), 803-813.

Bennett, K. (1992). Feminist bisexuality: A both/and option for an either/or world. In E. R. Weise (Ed.), Close to home: Bisexuality and feminism (pp. 205-231). Seattle, WA: The Seal Press.

Bennett, L. (2019, May 30). Budget 2019: Massive boost for mental health. NZ Herald. Retrieved from https://www.nzherald.co.nz/nz/news/article.cfm?c_id=1\&objectid=12235563

Berger, J. C., Green, R., Laub, D. R., Reynolds, C. L., Walker, P. A., \& Wollman, L. 
(1977). Standards of care: The hormonal and surgical sex reassignment of gender dysphoric persons (Version 1). Galveston, Texas: University of Texas Medical Branch, Janus Information Center.

Berger, J. C., Green, R., Laub, D. R., Reynolds, C. L., Walker, P. A., \& Wollman, L. (1980). Standards of care: The hormonal and surgical sex reassignment of gender dysphoric persons (Version 2). San Francisco, California: Harry Benjamin International Gender Dysphoria Association.

Berger, J. C., Green, R., Laub, D. R., Reynolds, C. L., Walker, P. A., \& Wollman, L. (1981). Standards of care: The hormonal and surgical sex reassignment of gender dysphoric persons (Version 3). San Francisco, California: Harry Benjamin International Gender Dysphoria Association.

Berger, J. C., Green, R., Laub, D. R., Reynolds, C. L., Walker, P. A., \& Wollman, L. (1990). Standards of care: The hormonal and surgical sex reassignment of gender dysphoric persons (Version 4). Palo Alto, California: Harry Benjamin International Gender Dysphoria Association.

Berger, R. (2015). Now I see it, now I don't: researcher's position and reflexivity in qualitative research. Qualitative Research, 15(2), 219-234.

Bergler, E. (1956). Homosexuality: Disease of way of life? New York: Hill \& Wang.

Bergold, J., \& Thomas, S. (2012). Participatory research methods: A methodological approach in motion. Historical Social Research, 37(4), 191-222.

Bieber, I., Dain, H. J., Dince, P. R., Drellich, M. G., Grand, H. G., Gundlach, R. H., ... Bieber, T. B. (1962). Homosexuality: A psychoanalytic study. New York: Basic Books.

Birkenhead, A., \& Rands, D. (2012). Let's talk about sex... (sexuality and gender). Auckland: Retrieved from https://www.mentalhealth.org.nz/assets/ResourceFinder/Lets-talk-about-sex.pdf

Bizic, M. R., Jeftovic, M., Pusica, S., Stojanovic, B., Duisin, D., Vujovic, S., ... Djordjevic, M. L. (2018). Gender dysphoria: Bioethical aspects of medical treatment. BioMed Research International, 9652305, 1-6. 
Blair, K. L. (2016). Ethical research with sexual and gender minorities. In A. E. Goldberg (Ed.), The SAGE encyclopedia of LGBTQ studies (pp. 375-380). Thousand Oaks: Sage.

Bockting, W. O., Miner, M. H., Swinburne Romine, R. E., Hamilton, A., \& Coleman, E. (2013). Stigma, mental health, and resilience in an online sample of the US transgender population. American Journal of Public Health, 103(5), 943-951.

Bockting, W. O., Robinson, B., Benner, A., \& Scheltema, K. (2004). Patient satisfaction with transgender health services. Journal of Sex \& Marital Therapy, 30(4), 277294.

Bontempo, D. E., And, M. A., \& D’augelli, A. R. (2002). Effects of at-school victimization and sexual orientation on lesbian, gay, or bisexual youths' health risk behavior. Journal of Adolescent Health, 30, 364-374.

Bordin, E. S. (1979). The generalizability of the psychoanalytic concept of the working alliance. Psychotherapy: Theory, Research \& Practice, 16(3), 252-260.

Bouman, W. P., Bauer, G. R., Richards, C., \& Coleman, E. (2010). World Professional Association for Transgender Health consensus statement on considerations of the role of distress (criterion D) in the DSM diagnosis of gender identity disorder. International Journal of Transgenderism, 12(2), 100-106.

Bowers, R., Plummer, D., \& Minichiello, V. (2005). Homophobia in counselling practice. International Journal for the Advancement of Counselling, 27(3), 471489.

Brachman, L. (2011). Treating the student's body: A cultural competency case study of LGBT patients and their providers on a college campus. New Jersey: Retrieved from http://www.princeton.edu/ pphr/lauren_brachman_11.pdf

Bradford, J., Reisner, S. L., Honnold, J. A., \& Xavier, J. (2013). Experiences of transgender-related discrimination and implications for health: Results from the Virginia transgender health initiative study. American Journal of Public Health, 103(10), 1820-1829.

Braun, V., \& Clarke, V. (2006). Using thematic analysis in psychology. Qualitative 
Research in Psychology, 3, 77-101.

Braun, V., \& Clarke, V. (2013). Successful qualitative research: A practical guide for beginners. London: Sage.

Braun, V., \& Clarke, V. (2019a). To saturate or not to saturate? Questioning data saturation as a useful concept for thematic analysis and sample-size rationales. Qualitative Research in Sport, Exercise and Health.

Braun, V., \& Clarke, V. (2019b). Answers to frequently asked questions about thematic analysis. Retrieved from https://cdn.auckland.ac.nz/assets/psych/about/ourresearch/documents/Answers\%20to\%20frequently\%20asked\%20questions \%20abo ut\%20thematic\%20analysis\%20April\%202019.pdf

Braun, V., Clarke, V., Terry, G., \& Hayfield, N. (2019). Thematic analysis. In P. Liamputtong (Ed.), Handbook of research methods in health and social sciences (pp. 843-860). Singapore: Springer.

Brewster, M. E., Moradi, B., Deblaere, C., \& Velez, B. L. (2013). Navigating the borderlands: The roles of minority stressors, bicultural self-efficacy, and cognitive flexibility in the mental health of bisexual individuals. Journal of Counseling Psychology, 60, 543-556.

Bridges, D. (2001). The ethics of outsider research. Journal of the Philosophy of Education, 35(3), 371-386.

Britzman, D. P. (1995). Is there a queer pedagogy? Or, stop reading straight. Educational Theory, 45(2), 151-165.

Brooks, V. R. (1981). Minority stress and lesbian women. Lexington: Lexington Books.

Brotto, L. A., Knudson, G., Inskip, J., Rhodes, K., \& Erskine, Y. (2010). Asexuality: A mixed-methods approach. Archives of Sexual Behavior, 39(3), 599-618.

Broughton, C. (2016, October 22). Thousands of Kiwi kids waiting for mental health treatment. Stuff. Retrieved from https://www.stuff.co.nz/national/health/85509692/thousand-of-kiwi-kids-waitingfor-mental-health-treatment 
Broughton, C. (2017, April 11). DHB, psychiatrist fail man who took his own life while in care. Stuff. Retrieved from https://www.stuff.co.nz/national/health/91449462/dhb-psychiatrist-fail-youngman-in-care

Brown, K. (2017, June 7). Govt under fire over mental health spending. RNZ News. Retrieved from https://www.rnz.co.nz/news/political/332501/govt-under-fire-overmental-health-spending

Brunton, W. (2011). Mental health services. Retrieved from http://www.teara.govt.nz/en/mental-health-services

Budge, S. L., Adelson, J. L., \& Howard, K. A. S. (2013). Anxiety and depression in transgender individuals: The roles of transition status, loss, social support, and coping. Journal of Consulting and Clinical Psychology, 81(3), 545-557.

Bullock, J. (2019, August). Role of the mental health professional in supporting physical transition options for trans and non-binary clients. Presentation at the Rainbow Cultural Competency Workshop, Wellington.

Burckell, L. A., \& Goldfried, M. R. (2006). Therapist qualities preferred by sexualminority individuals. Psychotherapy, 43(1), 32-49.

Burgess, R. G. (1984). In the field: An introduction to field research. New York: Routledge.

Burton, C. M., Marshal, M. P., Chisolm, D. J., Sucato, G. S., \& Friedman, M. S. (2013). Sexual minority-related victimization as a mediator of mental health disparities in sexual minority youth: A longitudinal analysis. Journal of Youth and Adolescence, 42(3), 394-402.

Butler, J. (1990). Gender trouble. London: Routledge.

Butler, J. (1991). Imitation and gender insubordination. In D. Fuss (Ed.), Inside/Out: Lesbian Theories, Gay Theories (pp. 13-31). New York: Routledge

Callahan, C. (2019). In which I fail to find you any gate-keeping. Retrieved from https://medium.com/@mariacatt42/in-which-i-fail-to-find-you-any-gate-keeping- 
$622 c 447 a 835 f$

Campbell, G. (2017, August 31). Gordon Campbell on the funding crisis in the public health system. Werewolf. Retrieved from http://werewolf.co.nz/2017/08/gordoncampbell-on-the-funding-crisis-in-the-public-health-system/

Cann, G. (2017, March 1). Nine in 10 healthcare workers feel understaffed and underresourced. Stuff. Retrieved from https://www.stuff.co.nz/national/health/89932971/nine-in-10-healthcare-workersfeel-understaffed-andunderresourced?link_id=0\&can_id=4de9a124d87887b05275aece18e8aec1\&source =email-what-a-year-6\&email_referrer=\&email_subject=what-a-year

Carr, L. T. (1994). The strengths and weaknesses of quantitative and qualitative research: What method for nursing? Journal of Advanced Nursing, 20(20), 716721.

Castleden, H., Morgan, V. S., \& Lamb, C. (2012). "I spent the first year drinking tea": Exploring Canadian university researchers' perspectives on community-based participatory research involving Indigenous peoples. The Canadian Geographer, 56(2), 160-179.

Cava, P. (2016). Cisgender and Cissexual. In N. A. Naples (Ed.), The Wiley Blackwell Encyclopedia of Gender and Sexuality Studies, (pp. 1-4). Malden, MA: Wiley Blackwell.

Cavanaugh, T., Hopwood, R., \& Lambert, C. (2016). Informed consent in the medical care of transgender and gender-nonconforming patients. AMA Journal of Ethics, 18(11), 1147-1155.

Chevrette, R. (2013). Outing heteronormativity in interpersonal and family communication: Feminist applications of queer theory "beyond the sexy streets". Communication Theory, 23(2), 170-190.

Chiang, S. Y., Fleming, T., Lucassen, M., Fenaughty, J., Clark, T., \& Denny, S. (2017). Mental health status of double minority adolescents: Findings from national crosssectional health surveys. Journal of Immigrant and Minority Health, 19(3), 499- 
510.

Civil Union Act, No. 102. (2004). Retrieved from

http://www.legislation.govt.nz/act/public/2004/0102/latest/whole.html

Clark, D. (2017, May 5). Mental health waiting times a growing concern. New Zealand Labour Party. Retrieved from

https://www.labour.org.nz/mental_health_waiting_times_a_growing_concern

Clark, T. C., Lucassen, M. F. G., Bullen, P., Denny, S. J., Fleming, T. M., Robinson, E. M., \& Rossen, F. V. (2014). The health and well-being of transgender high school students: Results from the New Zealand adolescent health survey (youth'12). Journal of Adolescent Health, 55(1), 93-99.

Clarke, V., Ellis, S. J., Peel, E., \& Riggs, D. W. (2010). Lesbian, gay, bisexual, trans and queer psychology: An introduction. Cambridge: Cambridge University Press.

Clayton, R. (2017, March 29). Mental health nurses are "burnt out" while patients miss out. Stuff. Retrieved from https://www.stuff.co.nz/national/health/90963418/mental-health-nurses-are-burntout-while-patients-miss-out

Clunie, M., RainbowYOUTH, InsideOUT, Intersex Trust of Aotearoa New Zealand, Tīwhanawhana, Love Life Fono, ... Mental Health Foundation of New Zealand. (2018). Rainbow communities, mental health and addictions: A submission to the Government Inquiry into Mental Health and Addiction - Oranga Tāngata, Oranga Whānau. Retrieved from https://www.mentalhealth.org.nz/assets/OurWork/policy-advocacy/Rainbow-communities-and-mental-health-submission-tothe-Inquiry-into-Mental-Health-and-Addiction-08062018.pdf

Cochran, S. D. (2001). Emerging issues in research on lesbians' and gay men's mental health: Does sexual orientation really matter? The American Psychologist, 56(11), 931-947.

Cochran, S. D., \& Mays, V. M. (2000). Lifetime prevalence of suicide symptoms and affective disorders among men reporting same-sex sexual partners: Results from NHANES III. American Journal of Public Health, 90(4), 573-578. 
Cohen-Kettenis, P. T. (2001). Gender identity disorder in DSM? Journal of the American Academy of Child and Adolescent Psychiatry, 40(4), 391.

Cohen-Kettenis, P. T., \& Pfäfflin, F. (2010). The DSM diagnostic criteria for gender identity disorder in adolescents and adults. Archives of Sexual Behavior, 39(2), 499-513.

Coleman, E., Bockting, W., Botzer, M., Cohen-Kettenis, P., DeCuypere, G., Feldman, J., ... Zucker, K. (2012). Standards of care for the health of transsexual, transgender, and gender-nonconforming people (Version 7). International Journal of Transgenderism, 13, 165-232.

Compton, J. (2019, January 15). "Pro-lesbian” or "trans-exclusionary”? Old animosities boil into public view. NBC News. Retrieved from https://www.nbcnews.com/feature/nbc-out/pro-lesbian-or-trans-exclusionary-oldanimosities-boil-public-view-n958456

Conrad, P., \& Angell, A. (2004). Homosexuality and remedicalization. Society, 41(5), $32-39$

Corliss, H., Belzer, M., Forbes, C., \& Wilson, E. (2007). An evaluation of service utilization among male to female transgender youth: Qualitative study of a clinicbased sample. Journal of LGBT Health Research, 3(2), 49-61.

Couch, M., Pitts, M., Mulcare, H., Croy, S., Mitchell, A., \& Patel, S. (2007). TranzNation: A report on the health and wellbeing of transgendered people in Australia and New Zealand. Melbourne: Australian Research Centre in Sex, Health and Society, La Trobe University.

Coughlan, T. (2018, October 16). Gender affirmation surgery cap lifted. Newsroom. Retrieved from https://www.newsroom.co.nz/2018/10/15/277680/genderreassignment-surgery-cap-lifted

Cowlishaw, S. (2017, March 31). Auckland's crumbling mental health services. Newsroom. Retrieved from https://www.newsroom.co.nz/@health-science/2017/03/30/17370/auckland-mental-health-services-crumbling

Crenshaw, K. (1989). Demarginalizing the intersection of race and sex: A black 
feminist critique of antidiscrimination doctrine, feminist theory and antiracist politics. University of Chicago Legal Forum, 1989, 139-168.

Crenshaw, K. (1991). Mapping the margins: Intersectionality, identity politics, and violence against women of color. Stanford Law Review, 43(6), 1241-1299.

Creswell, J. W., \& Plano Clark, V. L. (2007). Designing and Conducting Mixed Methods Research. Los Angeles: Sage.

Creswell, J. W., Plano Clark, V. L., Gutmann, M. L., \& Hanson, W. E. (2003). Advanced mixed methods research designs. In A. Tashakkori \& C. Teddlie (Eds.), Handbook of mixed methods in social and behavioral research (pp. 209-240). Thousand Oaks, CA: Sage.

Cunliffe, A. L., \& Karunanayake, G. (2013). Working within hyphen-spaces in ethnographic research: Implications for research identities and practice. Organizational Research Methods, 16(3), 364-392.

Daley, A. (2010). Being recognized, accepted, and affirmed: Self-disclosure of lesbian/queer sexuality within psychiatric and mental health service settings. Social Work in Mental Health, 8(4), 336-355.

Daly, M. (2019, May 31). Rainbow Youth welcome Government funding for gender affirming surgery. Stuff. Retrieved from https://www.stuff.co.nz/national/politics/113159788/rainbow-youth-welcomegovernment-funding-for-gender-affirming-surgery

Davies, A., Bouman, W. P., Richards, C., Barrett, J., Ahmad, S., Baker, K., ... Stradins, L. (2013). Patient satisfaction with gender identity clinic services in the United Kingdom. Sexual and Relationship Therapy, 28(4), 400-418.

Davy, Z. (2015). The DSM-5 and the politics of diagnosing transpeople. Archives of Sexual Behavior, 44(5), 1165-1176.

Deguara, B. (2019, May 30). Budget NZ 2019: \$1.9 billion won’t fix NZ's mental health crisis, psychologist says. Stuff. Retrieved from https://www.stuff.co.nz/business/budget/113130791/budget-nz-2019-19-billionwont-fix-nzs-mental-health-crisis-psychologist-says 
Delahunt, J. W., Denison, H. J., Sim, D. A., Bullock, J. J., \& Krebs, J. D. (2018). Increasing rates of people identifying as transgender presenting to endocrine services in the wellington region. New Zealand Medical Journal, 131(1468), 3342. Retrieved from https://www.nzma.org.nz/journal/read-the-journal/allissues/2010-2019/2018/vol-131-no-1468-19-january-2018/7463

Denzin, N. K. (2006). Analytic autoethnography, or déjà vu all over again. Journal of Comtemporary Ethnography, 35(4), 419-428.

Department of Internal Affairs. (2019). Information about changing sex/gender identity. Retrieved from https://www.passports.govt.nz/what-you-need-to-renew-or-applyfor-a-passport/information/

Deutsch, M. B. (2012). Use of the informed consent model in the provision of cross-sex hormone therapy: A survey of the practices of selected clinics. International Journal of Transgenderism, 13(3), 140-146.

Deutsch, M. B. (2019). Information on estrogen hormone therapy. Retrieved from https://transcare.ucsf.edu/article/information-estrogen-hormone-therapy

Dhejne, C., Lichtenstein, P., Boman, M., Johansson, A. L. V., Långström, N., \& Landén, M. (2011). Long-term follow-up of transsexual persons undergoing sex reassignment surgery: Cohort study in Sweden. PLoS ONE, 6(2), e16885.

Diamond, L. M. (2003). Integrating research on sexual-minority and heterosexual development: theoretical and clinical implications. Journal of Clinical Child and Adolescent Psychology, 32(4), 490-535.

Dickinson, P., \& Adams, J. (2014). Resiliency and mental health and well-being among lesbian, gay and bisexual people. International Journal of Mental Health Promotion, 16(2), 117-125.

Dimitriadis, G. (2001). Coming clean at the hyphen: Ethics and dialogue at a local community center. Qualitative Inquiry, 7(5), 578-597.

Dobinson, C., \& Macdonnell, J. (2005). Improving the access and quality of public health services for bisexuals. Journal of Bisexuality, 5(7), 39-77. 
Dodge, B., \& Sandfort, T. G. M. (2007). A review of mental health research on bisexual individuals when compared to homosexual and heterosexual individuals. In B. A. Firestein (Ed.), Becoming visible : counseling bisexuals across the lifespan (pp. 28-51). New York, NY: Columbia University Press.

Dorey, P. (2006). Homosexual law reform. In P. Dorey (Ed.), The Labour Governments 1964-1970 (pp. 345-358). London: Routledge.

Dorland, J. M., \& Fischer, A. R. (2001). Gay, lesbian, and bisexual individuals' perceptions: An analogue study. The Counseling Psychologist, 29(4), 532-547.

Drescher, J. (2008). A history of homosexuality and organized psychoanalysis. The Journal of the American Academy of Psychoanalysis and Dynamic Psychiatry, $36(3), 443-460$.

Drescher, J., Haller, E., \& American Psychiatric Association Caucus of Lesbian, Gay and Bisexual Psychiatrists. (2012). Position statement on access to care for transgender and gender variant individuals. Arlington, VA: American Psychiatric Association.

Drescher, J., Schwartz, A., Casoy, F., McIntosh, C. A., Hurley, B., Ashley, K., ... Tompkins, D. A. (2016). The growing regulation of conversion therapy. Journal of Medical Regulation, 102(2), 7-12.

Duff, M. (2017, July 23). Cancer treatment has become a "postcode lottery", say campaigners. Stuff. Retrieved from https://www.stuff.co.nz/national/health/94993641/cancer-treatment-has-become-apostcode-lottery-say-campaigners

Durie, M. (1994). Whaiora: Māori health development. Auckland: Oxford University Press.

Dwyer, S. C., \& Buckle, J. L. (2009). The space between: On being an insider-outsider in qualitative research. International Journal of Qualitative Methods, 8(1), 54-63.

Eady, A., Dobinson, C., \& Ross, L. E. (2011). Bisexual people's experiences with mental health services: A qualitative investigation. Community Mental Health Journal, 47(4), 378-389. 
Easton, B. (2002). The New Zealand health reforms of the 1990s in context. Applied Health Economics and Health Policy, 1(2), 107-112.

Edwards, J., \& Van Roekel, H. (2009). Gender, sexuality and embodiment: Access to and experience of healthcare by same-sex attracted women in Australia. Current Sociology, 57(2), 193-210.

Edwards, K., Lund, C., Mitchell, S., \& Andersson, N. (2008). Trust the process: Community-based researcher partnerships. Pimatisiwin, 6(2), 186-199.

Ellard-Gray, A., Jeffrey, N. K., Choubak, M., \& Crann, S. E. (2015). Finding the hidden participant: Solutions for recruiting hidden, hard-to-reach, and vulnerable populations. International Journal of Qualitative Methods, 14(5), 1-10.

Elliott, M. (2017). People's Mental Health Report. Wellington: ActionStation.

Ellis, S. J., Bailey, L., \& McNeil, J. (2015). Trans people's experiences of mental health and gender identity services: A UK study. Journal of Gay \& Lesbian Mental Health, 19(1), 4-20.

Engdahl, U. (2014). Wrong body. Transgender Studies Quarterly, 1(1-2), 267-269.

Evans, M., \& Barker, M. J. (2010). How do you see me? Coming out in counselling. British Journal of Guidance and Counselling, 38(4), 375-391.

Farvid, P. (2018). The psychology of modern racism. NZ Herald. Retrieved from https://www.nzherald.co.nz/nz/news/article.cfm?c_id=1\&objectid=12035625

Feilzer, M. Y. (2009). Doing mixed methods research pragmatically: Implications for the rediscovery of pragmatism as a research paradigm. Journal of Mixed Methods Research, 4(1), 6-16.

Fergusson, D. M., Horwood, L. J., \& Beautrais, A. L. (1999). Is sexual orientation related to mental health problems and suicidality in young people? Archives of General Psychiatry, 56(10), 876-880.

Fergusson, D. M., Horwood, L. J., Ridder, E. M., \& Beautrais, A. L. (2005). Sexual orientation and mental health in a birth cohort of young adults. Psychological Medicine, 35(7), 971-981. 
Ferris, J. (2006). The nomenclature of the community: An activist's perspective. In M. D. Shankle (Ed.), The handbook of lesbian, gay, bisexual, and transgender public health: A practitioner's guide to service (pp. 3-10). Binghamton, NY: The Haworth Press.

Filice, E., \& Meyer, S. B. (2018). Patterns, predictors, and outcomes of mental health service utilization among lesbians, gay men, and bisexuals: A scoping review. Journal of Gay and Lesbian Mental Health, 22(2), 162-195.

Fine, M. (1994). Working the hyphens: Reinventing self and other in qualitative research. In N. K. Denzin \& Y. S. Lincoln (Eds.), Handbook of qualitative research (pp. 70-82). Thousand Oaks, CA: Sage.

Finlay, D. L., \& Gough, B. (2003). Reflexivity: A practical guide for researchers in health and social sciences. Oxford: Blackwell Science.

Finlay, L. (2002). "Outing” the researcher: The provenance, process, and practice of reflexivity. Qualitative Health Research, 12(4), 531-545.

Fisher, C. M., Irwin, J. A., \& Coleman, J. D. (2014). LGBT health in the midlands: A rural/urban comparison of basic health indicators. Journal of Homosexuality, 61(8), 1062-1090.

Forbat, L., \& Henderson, J. (2005). Theoretical and practical reflections on sharing transcripts with participants. Qualitative Health Research, 15(8), 1114-1128.

Ford, C. S., \& Beach, F. A. (1951). Patterns of sexual behavior. New York: Harper \& Row.

Forster, M. (2003). Te hoe nuku roa: A journey towards Maori centered research. Ethnobotany Research \& Applications, 1, 47-53.

Fotopoulou, A. (2012). Intersectionality queer studies and hybridity: Methodological frameworks for social research. Journal of International Women's Studies, 13(2), 19-32.

Fraser, G., Bulbulia, J., Greaves, L. M., Wilson, M. S., \& Sibley, C. G. (2019). Coding responses to an open-ended gender measure in a New Zealand national sample. 
Journal of Sex Research, 1-8.

Fraser, G., Shields, J. K., Brady, A., \& Wilson, M. (2018). The postcode lottery: Gender-affirming healthcare provision across New Zealand's District Health Boards. Unpublished manuscript, Victoria University of Wellington, Wellington, New Zealand.

Fraser, G. (2018). Evaluating inclusive gender identity measures for use in quantitative psychological research. Psychology and Sexuality, 9(4), 343-357.

Fraser, G., Wilson, M. S., Garisch, J. A., Robinson, K., Brocklesby, M., Kingi, T., ... Russell, L. (2017). Non-suicidal self-injury, sexuality concerns, and emotion regulation among sexually diverse adolescents: A multiple mediation analysis. Archives of Suicide Research, 22(3), 432-452.

Fredriksen-Goldsen, K. I., Cook-Daniels, L., Kim, H.-J., Erosheva, E. A., Emlet, C. A., Hoy-Ellis, C. P., ... Muraco, A. (2013). Physical and mental health of transgender older adults: An at-risk and underserved population. The Gerontologist, 54(3), $488-500$.

Fredriksen-Goldsen, K. I., Simoni, J. M., Kim, H. J., Lehavot, K., Walters, K. L., Yang, J., ... Muraco, A. (2014). The health equity promotion model: Reconceptualization of lesbian, gay, bisexual, and transgender (LGBT) health disparities. American Journal of Orthopsychiatry, 84(6), 653-663.

Freund, K., \& Pinkava, V. (1961). Homosexuality in man and its association with parental relationships. Review of Czechoslovak Medicine, 7, 32-40.

Friedman, M. S., Marshal, M. P., Guadamuz, T. E., Wei, C., Wong, C. F., Saewyc, E., \& Stall, R. (2011). A meta-analysis of disparities in childhood sexual abuse, parental physical abuse, and peer victimization among sexual minority and sexual nonminority individuals. American Journal of Public Health, 101(8), 1481-1494.

Fusch, P. I., \& Ness, L. R. (2015). Are we there yet? Data saturation in qualitative research. The Qualitative Report, 20(9), 1408-1416. 
Gamble Blakey, A., \& Treharne, G. J. (2019a). Advancing transgender healthcare teaching in Aotearoa/New Zealand. New Zealand Medical Journal, 132(1491), 104-106.

Gamble Blakey, A., \& Treharne, G. J. (2019b). Overcoming barriers to transgender healthcare education in Aotearoa New Zealand. New Zealand Journal of Educational Studies, 54(2), 357- 366.

Gamson, J. (1995). Must identity movements self-destruct? A queer dilemma. Social Problems, 42(3), 390-407.

Garofalo, R., Wolf, R. C., Kessel, S., Palfrey, S. J., \& DuRant, R. H. (1998). The association between health risk behaviors and sexual orientation among a schoolbased sample of adolescents. Pediatrics, 101(5), 895-902.

Gavey, N. (1989). Feminist poststructuralism and discourse analysis: Contributions to feminist psychology. Psychology of Women Quarterly, 13(4), 459-475.

Gender Minorities Aotearoa. (2017). Glossary of gender related terms and how to use them. Retrieved from https://genderminoritiesaotearoa.files.wordpress.com/2016/06/gender-minoritiesaotearoa-glossary-gender-minority-words-and-how-to-use-them.pdf

Genter, J. A. (2017, August 28). Suicide rate reflects our broken mental health system. Green Party of Aotearoa New Zealand. Retrieved from https://www.greens.org.nz/news/press-release/suicide-rate-reflects-our-brokenmental-health-system

Germon, J. (2009). Gender: A genealogy of an idea. New York: Palgrave Macmillan.

Giblon, R., \& Bauer, G. R. (2017). Health care availability, quality, and unmet need: A comparison of transgender and cisgender residents of Ontario, Canada. BMC Health Services Research, 17, 1-10.

Gittings, B. (2008). Show and tell. Journal of Gay \& Lesbian Mental Health, 12(3), 289-295.

GLAAD. (2017). Accelerating acceptance: A Harris Poll survey of Americans' 
acceptance of LGBTQ people. New York: Retrieved from https://www.glaad.org/files/aa/2017_GLAAD_Accelerating_Acceptance.pdf

Goettsche, R. S. (2015). Lesbian, gay and bisexual client experiences and their therapeutic practice with sexual minorities: An interpretative phenomenological analysis. (Doctoral thesis, Pacifica Graduate Institute). Retrieved from https://search.proquest.com/docview/1658104167?pq-origsite=gscholar

Golding, J. (1997). Without prejudice: Mind lesbian, gay and bisexual mental health awareness research. London: Mind Publications.

Goodwin, E. (2016, September 23). Operations a "postcode lottery." Otago Daily Times. Retrieved from https://www.odt.co.nz/news/dunedin/health/operationspostcode-lottery

Goodwin, E. (2017, April 24). Mental health wait up to two months. Otago Daily Times. Retrieved from https://www.odt.co.nz/news/dunedin/health/mental-healthwait-two-months

Gooren, L. J. (2011). Care of transsexual persons. New England Journal of Medicine, 364(13), 1251-1257.

Government Inquiry into Mental Health and Addiction (2018). He Ara Oranga: Report of the Government Inquiry into Mental Health and Addiction. Wellington: Retrieved from https://mentalhealth.inquiry.govt.nz/assets/Summary-reports/HeAra-Oranga.pdf

Graham-McLay, C. (2018, September 26). Jacinda Ardern's progressive politics made her a global sensation. But do they work at home? New York Times. Retrieved from https://www.nytimes.com/2018/09/26/world/asia/jacinda-ardern-un-newzealand.html

Granek, L. (2013). Putting ourselves on the line: The epistemology of the hyphen, intersubjectivity and social responsibility in qualitative research. International Journal of Qualitative Studies in Education, 26(2), 178-197.

Grant, C., \& Osanloo, A. (2014). Understanding, selecting, and integrating a theoretical framework in dissertation research: Creating the blueprint for your "house". 
Administrative Issues Journal Education Practice and Research, 4(2), 12-26.

Grant, J. M., Mottet, L. A., Tanis, J., With, D. M., Herman, J. L., Harrison, J., \&

Keisling, M. (2010). National transgender discrimination report on health and health care. Washington, DC: National Center for Transgender Equality and the National Gay and Lesbian Task Force.

Greaves, L. M., Barlow, F. K., Huang, Y., Stronge, S., Fraser, G., \& Sibley, C. G. (2017). Asexual identity in a New Zealand national sample: Demographics, wellbeing, and health. Archives of Sexual Behavior, 46(8), 2417-2427.

Grimshaw, P. (2013). Women's suffrage in New Zealand. Auckland: Auckland University Press.

Grove, J. (2009). How competent are trainee and newly qualified counsellors to work with lesbian, gay, and bisexual clients and what do they perceive as their most effective learning experiences? Counselling \& Psychotherapy Research, 9(2), 7885.

Grove, J. (2017). Researching a marginalised group: Reflections on being an outsider. Counselling and Psychotherapy Research, 17(3), 176-180.

Haas, A. P., Eliason, M., Mays, V. M., Mathy, R. M., Cochran, S. D., \& Anthony, R. (2011). Suicide and suicide risk in lesbian, gay, bisexual, and transgender populations: Review and recommendations. Journal of Homosexuality, 58(1), 1051.

Haldeman, D. C. (1994). The practice and ethics of sexual orientation conversion therapy. Journal of Consulting and Clinical Psychology, 62(2), 221-227.

Halperin, D. M. (2003). The normalization of queer theory. Journal of Homosexuality, 45(2-4), 339-343.

Halpern, A. L. (2011). The proposed diagnosis of hypersexual disorder for inclusion in DSM-5: Unnecessary and harmful. Archives of Sexual Behavior, 40(3), 487-488.

Hanson, W. E., Creswell, J. W., Plano Clark, V. L., Petska, K. S., \& Creswell, J. D. (2005). Mixed methods research designs in counseling psychology. Journal of 
Counseling Psychology, 52(2), 224-235.

Happell, B., \& Scholz, B. (2018). Doing what we can, but knowing our place: Being an ally to promote consumer leadership in mental health. International Journal of Mental Health Nursing, 27(1), 440-447.

Harnett, P., O'Donovan, A., \& Lambert, M. J. (2010). The dose response relationship in psychotherapy: Implications for social policy. Clinical Psychologist, 14(2), 39-44.

Harrison, J., MacGibbon, L., \& Morton, M. (2001). Regimes of trustworthiness in qualitative research: The rigors of reciprocity. Qualitative Inquiry, 7(3), 323-345.

Hatzenbuehler, M. L. (2010). Social factors as determinants of mental health disparities in LGB populations: Implications for public policy. Social Issues and Policy Review, 4(1), 31-62.

Hayfield, N., \& Huxley, C. (2015). Insider and outsider perspectives: Reflections on researcher identities in research with lesbian and bisexual women. Qualitative Research in Psychology, 12(2), 91-106.

Health and Disability Commissioner Act, No. 88. (1994). Retrieved from http://legislation.govt.nz/act/public/1994/0088/latest/DLM333584.html

Healthpoint. (2019). Mental Health \& Addictions. Retrieved from https://www.healthpoint.co.nz/mental-healthaddictions/?programmeArea=im\%3A645234

Hellawell, D. (2006). Inside-out: Analysis of the insider-outsider concept as a heuristic device to develop reflexivity in students doing qualitative research. Teaching in Higher Education, 11(4), 483-494.

Henrickson, M., Neville, S., Jordan, C., \& Donaghey, S. (2007). Lavender Islands: The New Zealand study. Journal of Homosexuality, 53(4), 223-248.

Herek, G. M. (1990). The context of anti-gay violence: Notes on cultural and psychological heterosexism. Journal of Interpersonal Violence, 5(3), 316-333.

Heron, J., \& Reason, P. (2001). The practice of co-operative inquiry: Research "with" rather than "on" people. In P. Reason \& H. Bradbury (Eds.), Handbook of action 
research: Participative inquiry and practice (pp. 179-188). London: Sage.

Hetrick, S. E., Bailey, A. P., Smith, K. E., Malla, A., Mathias, S., Singh, S. P., ... McGorry, P. D. (2017). Integrated (one-stop shop) youth health care: Best available evidence and future directions. The Medical Journal of Australia, 207(10), S5-S18.

Hiestand, K. R., Horne, S. G., \& Levitt, H. M. (2007). Effects of gender identity on experiences of healthcare for sexual minority women. Journal of LGBT Health Research, 3(4), 15-27.

Hines, M., Ahmed, S. F., \& Hughes, I. A. (2003). Psychological outcomes and genderrelated development in complete androgen insensitivity syndrome. Archives of Sexual Behavior, 32(2), 93-101.

Hodgetts, D., Chamberlain, K., Groot, S., \& Tankel, Y. (2014). Urban poverty, structural violence and welfare provision for 100 families in Auckland. Urban Studies, 51(10), 2036-2051.

Hoffman, N. D., Freeman, K., \& Swann, S. (2009). Healthcare preferences of lesbian, gay, bisexual, transgender and questioning youth. Journal of Adolescent Health, 45(3), 222-229.

Holden, M. (2016, December 15). Three surgeries every two years: NZ's shameful 50year waitlist for gender reassignment surgery. The Spinoff. Retrieved from https://thespinoff.co.nz/society/15-12-2016/three-surgeries-every-two-years-nzsshameful-50-year-waitlist-for-gender-reassignment-surgery/

Holt, V., Skagerberg, E., \& Dunsford, M. (2016). Young people with features of gender dysphoria: Demographics and associated difficulties. Clinical Child Psychology and Psychiatry, 21(1), 108-118.

Hooker, E. (1957). The adjustment of the male overt homosexual. Journal of Projective Techniques, 21(1), 18-31.

hooks, B. (1990). Marginality as a site of resistance. In R. Ferguson (Ed.), Out there: Marginalization and contemporary cultures (pp. 341-343). Cambridge, MA: MIT Press. 
Hopkinson, R. A., Keatley, E., Glaeser, E., Erickson-Schroth, L., Fattal, O., \& Nicholson Sullivan, M. (2017). Persecution experiences and mental health of LGBT asylum seekers. Journal of Homosexuality, 64(12), 1650-1666.

Horowitz, C. R., Robinson, M., \& Seifer, S. (2009). Community-based participatory research from the margin to the mainstream: Are researchers prepared? Circulation, 119(19), 2633-2642.

Hughes, T. L., Haas, A. P., Razzano, L., Cassidy, R., \& Matthews, A. (2000). Comparing lesbians' and heterosexual women's mental health: A multi-site survey. Journal of Gay and Lesbian Social Services, 11(1), 57-76.

Human Rights Act, No. 82. (1993). Retrieved from http://www.legislation.govt.nz/act/public/1993/0082/latest/DLM304212.html

Human Rights Commission. (2008). To be who I am: Report of the Inquiry into Discrimination Experienced by Transgender People. Auckland: Retrieved from https://www.hrc.co.nz/files/5714/2378/7661/15-Jan-2008_14-5648_HRC_Transgender_FINAL.pdf

Human Rights Commission. (2010). Human Rights In New Zealand. Auckland: Retrieved from https://www.hrc.co.nz/files/7014/2388/0544/Human_Rights_Review_2010_Full.p df

Hunt, B., Matthews, C., Milsom, A., \& Lammel, J. A. (2006). Lesbians with physical disabilities: A qualitative study of their experiences with counseling. Journal of Counseling \& Development, 84(2), 163-173.

Hutton, C. (2017, October 16). Staff shortages hit Wgtn mental health services. RNZ News. Retrieved from https://www.rnz.co.nz/news/national/341640/staffshortages-hit-wgtn-mental-health-services

InsideOUT. (2016). Making schools safer for trans and gender diverse youth. Wellington: InsideOUT.

InsideOUT. (2017). Legal rights at school for young people of minority sexualities and genders. Wellington: InsideOUT. 
Israel, B. A., Krieger, J., Vlahov, D., Ciske, S., Foley, M., Fortin, P., ... Tang, G. (2006). Challenges and facilitating factors in sustaining community-based participatory research partnerships: lessons learned from the Detroit, New York City and Seattle Urban Research Centers. Journal of Urban Health, 83(6), 10221040.

Israel, B. A., Schulz, A. J., Parker, E. A., \& Becker, A. B. (1998). Review of community-based research: Assessing partnership approaches to improve public health. Annual Review of Public Health, 19(1), 173-202.

Israel, T., Gorcheva, R., Burnes, T. R., \& Walther, W. A. (2008). Helpful and unhelpful therapy experiences of LGBT clients. Psychotherapy Research, 18(3), 294-305.

Jacquez, F., Vaughn, L. M., \& Wagner, E. (2013). Youth as partners, participants or passive recipients: A review of children and adolescents in community-based participatory research (CBPR). American Journal of Community Psychology, 51(1-2), 176-189.

Jagose, A. (1996). Queer theory: An introduction. New York: New York University Press.

James, S. E., Herman, J. L., Rankin, S., Keisling, M., Mottet, L., \& Anafi, M. (2016). The Report of the 2015 U.S. Transgender Survey. Washington, DC: National Center for Transgender Equality.

James, T., \& Platzer, H. (1999). Ethical considerations in qualitative research with vulnerable groups: Exploring lesbians' and gay men's experiences of health care A personal perspective. Nursing Ethics, 6(1), 73-81.

Johnson, A. H. (2015). Normative accountability: How the medical model influences transgender identities and experiences. Sociology Compass, 9(9), 803-813.

Johnson, J. L., Bottorff, J. L., Browne, A. J., Grewal, S., Hilton, B. A., \& Clarke, H. (2004). Othering and being othered in the context of health care services. Health Communication, 16(2), 255-271.

Johnson, R. B., \& Onwuegbuzie, A. J. (2004). Mixed methods research: A research paradigm whose time has come. Educational Researcher, 33(7), 14-26. 
Jones, A. (2012). Dangerous liaisons: Pakeha, kaupapa Maori, and educational research. New Zealand Journal of Educational Studies, 47(2), 100-112.

Jones, M. A., Botsko, M., \& Gorman, B. S. (2003). Predictors of psychotherapeutic benefit of lesbian, gay, and bisexual clients: The effects of sexual orientation matching and other factors. Psychotherapy: Theory, Research, Practice, Training, 40(4), 289-301.

Kamens, S. R. (2011). On the proposed sexual and gender identity diagnoses for DSM5: History and controversies. The Humanistic Psychologist, 39(1), 37-59.

Kameny, F. (2009). How it all started. Journal of Gay \& Lesbian Mental Health, 13(2), 76-81.

Kanuha, V. K. (2000). "Being" native versus "going native": Conducting social work research as an insider. Social Work, 45(5), 439-447.

Karkazis, K. (2008). Fixing sex: Intersex, medical authority, and lived experience. Durham: Duke University Press.

Kauffman, K. S. (1994). The insider/outsider dilemma: Field experience of a white researcher "getting in" a poor black community. Nursing Research, 43(3), 179183.

Kerekere, E. (2015). Takatāpui: Part of the whānau. Auckland: Tīwhanawhana Trust and Mental Health Foundation.

Kerekere, E. (2017a). Growing up Takatāpui: Whānau journeys. Auckland:

Tīwhanawhana Trust and RainbowYOUTH.

Kerekere, E. (2017b). Part of The Whānau: The Emergence of Takatāpui Identity - He Whāriki Takatāpui (Doctoral thesis, Victoria University of Wellington). Retrieved from

https://researcharchive.vuw.ac.nz/xmlui/bitstream/handle/10063/6369/thesis_acces s.pdf?sequence $=1$

Kidd, S. A., Veltman, A., Gately, C., Chan, K. J., \& Cohen, J. N. (2011). Lesbian, gay, and transgender persons with severe mental illness: Negotiating wellness in the 
context of multiple sources of stigma. American Journal of Psychiatric

Rehabilitation, 14(1), 13-39.

King, H. (2017, April 19). Cracks in NZ mental health system revealed in review | Stuff.co.nz. Stuff. Retrieved from https://www.stuff.co.nz/national/91655909/cracks-in-nz-mental-health-systemrevealed-in-review

King, M., Semlyen, J., Killaspy, H., Nazareth, I., \& Osborn, D. (2007). A systematic review of research on counselling and psychotherapy for lesbian, gay, bisexual \& transgender people. Leicestershire: British Association for Counselling and Psychotherapy.

King, M., McKeown, E., Warner, J., Ramsay, A., Johnson, K., Cort, C., ... Wright, L. (2003). Mental health and social wellbeing of gay men, lesbians and bisexuals in England and Wales: A summary of findings. London: Mind (National Association for Mental Health).

King, M., Semlyen, J., Tai, S. S., Killaspy, H., Osborn, D., Popelyuk, D., \& Nazareth, I. (2008). A systematic review of mental disorder, suicide, and deliberate self harm in lesbian, gay and bisexual people. BMC Psychiatry, 8, 1-17.

Kinsey, A. C., Pomeroy, W. B., \& Martin, C. E. (1948). Sexual behavior in the human male. Philadelphia: W. B. Saunders.

Kinsey, A. C., Pomeroy, W. B., \& Martin, C. E. (1953). Sexual behavior in the human female. Philadelphia: W. B. Saunders.

Kitzinger, C. (1999). Lesbian and gay psychology: Is it critical? Annual Review of Critical Psychology, 1, 50-66.

Knudson, G., De Cuypere, G., \& Bockting, W. (2010). Recommendations for revision of the DSM diagnoses of gender identity disorders: Consensus statement of the World Professional Association for Transgender Health. International Journal of Transgenderism, 12(2), 115-118.

Kondou, A. (2017, November). Practice Tips/Supporting Transgender Youth. Presentation at the National Youth Mental Health \& AOD Forum, Dunedin. 
Kong, T. S. K., Mahoney, D., \& Plummer, K. (2002). Queering the interview. In J. Gubrium \& J. Holstein (Eds.), Handbook of interview research: Context and method. London: Sage.

Kulick, D. (2000). Gay and lesbian language. Annual Review of Anthropology, 29, 243285.

Kuper, A., Lingard, L., \& Levinson, W. (2008). Critically appraising qualitative research. BMJ, 337, 404-407.

Kuper, L. E., Nussbaum, R., \& Mustanski, B. (2012). Exploring the diversity of gender and sexual orientation identities in an online sample of transgender individuals. Journal of Sex Research, 49(2-3), 244-254.

Kupers, T. A. (2005). Toxic masculinity as a barrier to mental health treatment in prison. Journal of Clinical Psychology, 61(6), 713-724.

Lambert, M. J., \& Barley, D. E. (2001). Research summary on the therapeutic relationship and psychotherapy outcome. Psychotherapy, 38, 357-361.

LaSala, M. C. (2003). When interviewing "family": Maximizing the insider advantage in the qualitative study of lesbians and gay men. Journal of Gay \& Lesbian Social Services, 15(1-2), 53-30.

Laurie, A. J. (2003). Lady-husbands and kamp ladies: Pre-1970 lesbian life in Aotearoa/New Zealand (Doctoral thesis, Victoria University of Wellington). Retrieved from https://researcharchive.vuw.ac.nz/bitstream/handle/10063/689/thesis.pdf?sequence $=1$

Leap, W., \& Boellstorf, T. (2004). Speaking in queer tongues: Globalization and gay language. Urbana: University of Illinois Press.

Lechner, M. E., Vogel, M. E., Garcia-Shelton, L. M., Leichter, J. L., \& Steibel, K. R. (1993). Self-reported medical problems of adult female survivors of childhood sexual abuse. Journal of Family Practice, 36(6), 633-638.

Lee, P. A., Nordenström, A., Houk, C. P., Ahmed, S. F., Auchus, R., Baratz, A., ... 
Witchel, S. (2016). Global disorders of sex development update since 2006:

Perceptions, approach and care. Hormone Research in Paediatrics, 85(3), 158180.

LeFebvre, L. E. (2017). Phantom lovers. Ghosting as a relationship dissolution strategy in the technological age. In N. M. Punyanunt-Carter \& J. S. Wrench (Eds.), The impact of social media in modern romantic relationships (pp. 219-236). New York, NY: Lexington Books.

Leonard, W., Lyons, A., \& Bariola, E. (2015). A Closer Look at Private Lives 2: Addressing the mental health and well-being of lesbian, gay, bisexual and transgender (LGBT) Australians. Melbourne: Australian Research Centre in Sex, Health \& Society, La Trobe University.

Lev, A. I. (2004). Transgender emergence: Therapeutic guidelines for working with gender-variant people and their families. New York: Routledge.

Lev, A. I. (2005). Disordering gender identity: Gender identity disorder in the DSM-IVTR. Journal of Psychology \& Human Sexuality, 174(3), 35-69.

Lev, A. I. (2016). Gender dysphoria. In N. Naples, R. C. Hoogland, M. Wikramasinghe, \& W. C. A. Wong (Eds.), The Wiley Blackwell encyclopedia of gender and sexuality studies (pp. 1-3). Singapore: Wiley-Blackwell.

Levin, R. (2014). Qualitative and quantitative evidence hierarchies: Mixing oranges and apples. Research and Theory for Nursing Practice, 28(2), 110-112.

Levin, S. (2019, September 3). “A critical point in history”: How Trump's attack on LGBT rights is escalating. The Guardian. Retrieved from https://www.theguardian.com/world/2019/sep/03/trump-attack-lgbt-rightssupreme-court

Levine, S. B., Brown, G. R., Coleman, E., Cohen-Kettenis, P. T., Van Maasdam, J., Pfäfflin, F., \& Schaefer, L. C. (1998). The standards of care for gender identity disorders (Version 5). Düsseldorf, Germany: Symposium Publishing.

Lewis, E., Vincent, B., Brett, A., Gibson, S., \& Walsh, R. J. (2017). I am your trans patient. $B M J, 357,1-3$. 
Liddle, B. J. (1996). Therapist sexual orientation, gender, and counseling practices as they relate to ratings on helpfulness by gay and lesbian clients. Journal of Counseling Psychology, 43(4), 394-401.

Liddle, B. J. (2000). Gay and lesbian clients' ratings of psychiatrists, psychologists, social workers, and counselors. Journal of Gay \& Lesbian Psychotherapy, 3(1), 81-93.

Liu, R. T., \& Mustanski, B. (2012). Suicidal ideation and self-harm in lesbian, gay, bisexual, and transgender youth. American Journal of Preventive Medicine, 42(3), $221-228$.

Low, J. (2019). A pragmatic definition of the concept of theoretical saturation. Sociological Focus, 52(2), 131-139.

Lucassen, M. F., Clark, T. C., Moselen, E., Robinson, E. M., \& Adolescent Health Research Group. (2014). Youth'12 the health and wellbeing of sexondary school students in New Zealand: Results for young people attracted to the same sex or both sexes. Auckland: Retrieved from http://oro.open.ac.uk/43995/1/Same Sex Report_14NM.pdf

Lucassen, M. F., Stasiak, K., Samra, R., Frampton, C. M., \& Merry, S. N. (2017). Sexual minority youth and depressive symptoms or depressive disorder: A systematic review and meta-analysis of population-based studies. Australian \& New Zealand Journal of Psychiatry, 51(8), 774-787.

Lucksted, A. (2004). Raising issues: Lesbian, gay, bisexual, and transgender people receiving services in the public mental health system. Baltimore: Center for Mental Health Services Research, University of Maryland.

Lysaght, Z. (2011, October). Epistemological and paradigmatic ecumenism in "Pasteur's Quadrant:" Tales from doctoral research. Paper presented at The Asian Conference on Education, Osaka. Retrieved from http://papers.iafor.org/wpcontent/uploads/conference-proceedings/ACE/ACE2011_proceedings.pdf

Macaulay, A. C., Commanda, L. E., Freeman, W. L., Gibson, N., McCabe, M. L., Robbins, C. M., \& Twohig, P. L. (1999). Participatory research maximises 
community and lay involvement. BMJ, 319, 774-778.

MacDonald, K. (2016, September 28). The stark reality: New Zealand no longer has a functioning Mental Health Service. Speaker. Retrieved from https://publicaddress.net/speaker/the-stark-reality-new-zealand-no-longer-has/

Mae, K. (n.d.). Here's the problem with performative allyship. Retrieved from https://www.scarymommy.com/performative-allyship-what-it-is-what-it-lookslike-and-why-we-want-to-avoid-it/

Mair, D. (2003). Gay men's experiences of therapy. Counselling and Psychotherapy Research, 3(1), 33-41.

Māori Dictionary. (2019). Te Aka online Māori dictionary. Retrieved from https://maoridictionary.co.nz/

Marriage (Definition of Marriage) Amendment Act, No. 20. (2013). Retrieved from http://www.legislation.govt.nz/act/public/2013/0020/latest/DLM4505003.html

Marshal, M. P., Dietz, L. J., Friedman, M. S., Stall, R., Smith, H. A., McGinley, J., ... Brent, D. A. (2011). Suicidality and depression disparities between sexual minority and heterosexual youth: A meta-analytic review. Journal of Adolescent Health, 49(2), 115-123.

Martin, H. (2017, September 22). Long waiting times for young people seeking mental health help. Stuff. Retrieved from https://www.stuff.co.nz/national/96989261/staffunder-pressure-creating-a-barrier-to-adequate-youth-mental-health-support-greenssay

Martin, H. (2019, August 19). District Health Board psychologists vote for further strike action, will not accept new patients. Stuff. Retrieved from https://www.stuff.co.nz/national/health/115119026/district-health-boardpsychologists-vote-for-further-strike-action-will-not-accept-new-patients

Mayock, P., Bryan, A., Carr, N., \& Kitching, K. (2008). Supporting LGBT lives: A study of mental health and well-being. Dublin: Retrieved from http://itgl.lu/wpcontent/uploads/2015/04/SB-2008-1.pdf 
Mays, V. M., \& Cochran, S. D. (2001). Mental health correlates of perceived discrimination among lesbian, gay, and bisexual adults in the United States. American Journal of Public Health, 91(11), 1869-1876.

McAllen, J. (2016, December 29). Turned away in a crisis. The Wireless. Retrieved from http://thewireless.co.nz/articles/turned-away-in-a-crisis

McAllen, J. (2017, September 13). Under pressure: Mental health workers give their view of the crisis. The Spinoff. Retrieved from https://thespinoff.co.nz/society/1309-2017/under-pressure-mental-health-workers-give-their-view-of-the-crisis/

McCabe, R., \& Priebe, S. (2004). The therapeutic relationship in the treatment of severe mental illness: A review of methods and findings. International Journal of Social Psychiatry, 50(2), 115-128.

McCall, L. (2005) The complexity of intersectionality. SIGNS: Journal of Women in Culture and Society, 30(31), 1771-1802.

McCann, E., Lee, R., \& Brown, M. (2016). The experiences and support needs of people with intellectual disabilities who identify as LGBT: A review of the literature. Research in Developmental Disabilities, 57, 39-53.

McCann, E., \& Sharek, D. (2014). Survey of lesbian, gay, bisexual, and transgender people's experiences of mental health services in Ireland. International Journal of Mental Health Nursing, 23(2), 118-127. https://doi.org/10.1111/inm.12018

McCord, J., McCord, W., \& Thurber, E. (1962). Some effects of paternal absence on male children. The Journal of Abnormal and Social Psychology, 64(5), 361-369.

McCullough, R., Dispenza, F., Parker, L. K., Viehl, C. J., Chang, C. Y., \& Murphy, T. M. (2017). The counseling experiences of transgender and gender nonconforming clients. Journal of Counseling \& Development, 95(4), 423-434.

McDonald, E., \& Byrne, J. (2015). The legal status of transsexual and transgender persons in Aotearoa New Zealand. In J. M. Scherpe (Ed.), The legal status of transsexual and transgender persons (pp. 527-568). Cambridge: Intersentia.

McFarlane, L. (1998). Diagnosis: Homophobic - The Experiences of Lesbians, Gay Men 
and Bisexuals in Mental Health Services. London: PACE.

McIntyre, J., Daley, A., Rutherford, K., \& Ross, L. E. (2011). Systems-level barriers in accessing supportive mental health services for sexual and gender minorities: Insights from the provider's perspective. Canadian Journal of Community Mental Health, 30(2), 173-186.

McNair, R. P., \& Hegarty, K. (2010). Guidelines for the primary care of lesbian, gay, and bisexual people: A systematic review. Annals of Family Medicine, 8(6), 533541.

McNeil, J., Bailey, L., Ellis, S., Morton, J., \& Regan, M. (2012). Trans Mental Health Study 2012. Edinburgh: Scottish Transgender Alliance.

Meier, C. (2017, June 5). Government's refusal to give Canterbury extra mental health funding under fire. Stuff. Retrieved from https://www.stuff.co.nz/national/health/94397701/governments-refusal-to-givecanterbury-extra-mental-health-funding-under-fire

Menninger, K. (1963). Introduction. In The Wolfenden report: Report of the committee on homosexual offenses and prostitution (Authorized American Edition). New York: Stein and Day.

Merriam, S. B., Johnson-Bailey, J., Lee, M. Y., Kee, Y., Ntseane, G., \& Muhamad, M. (2001). Power and positionality: Negotiating insider/outsider status within and across cultures. International Journal of Lifelong Education, 20(5), 405-416.

Merton, R. K. (1972). Insiders and outsiders: A chapter in the sociology of knowledge. American Journal of Sociology, 78(1), 9-47.

Meyer, I. H. (1995). Minority stress and mental health in gay men. Journal of Health and Social Behavior, 36(1), 38-56.

Meyer, I. H. (2003). Prejudice, social stress, and mental health in lesbian, gay, and bisexual populations: Conceptual issues and research evidence. Psychological Bulletin, 129(5), 674-697.

Meyer, W., Bockting, W. O., Cohen-Kettenis, P., Coleman, E., Diceglie, D., Devor, H., 
... Wheeler, C. C. (2001). The standards of care for gender identity disorders (Version 6). Journal of Psychology \& Human Sexuality, 13(1), 1-30.

Miller, T., \& Boulton, M. (2007). Changing constructions of informed consent: Qualitative research and complex social worlds. Social Science and Medicine, 65(11), 2199-2211.

Ministry for Culture and Heritage. (2014). Birth of the gay movement. Retrieved from https://nzhistory.govt.nz/culture/homosexual-law-reform/birth-of-the-gaymovement

Ministry of Health. (2015). Office of the Director of Mental Health annual report 2013. Wellington: Retrieved from http://www.health.qld.gov.au/mha2000/documents/annual-report-2013.pdf

Ministry of Health. (2016). How the electives process works. Retrieved from https://www.health.govt.nz/our-work/hospitals-and-specialist-care/electiveservices/how-electives-process-works

Ministry of Health. (2017). Annual update of key results 2016/17: New Zealand Health Survey. Retrieved from https://www.health.govt.nz/publication/annual-update-keyresults-2016-17-new-zealand-health-survey

Ministry of Health. (2018a). Briefing on gender reassignment surgery: Health report number 20180307. Wellington: Ministry of Health.

Ministry of Health. (2018b). Briefing on genital reassignment surgery: Initial advice on policy, funding and the engagement approach: Health report number 20180841. Wellington: Ministry of Health.

Ministry of Health. (2018c). High-cost treatment pool. Retrieved from https://www.health.govt.nz/our-work/hospitals-and-specialist-care/high-costtreatment-pool

Ministry of Health. (2018d). Gender reassignment surgery. Retrieved from http://www.health.govt.nz/our-work/preventative-health-wellness/deliveringhealth-services-transgender-people/gender-reassignment-surgery 
Ministry of Health. (2020). Abortion legislation: Information for health practitioners. Retrieved from https://www.health.govt.nz/our-work/regulation-health-anddisability-system/abortion-legislation-information-health-practitioners

Minkler, M. (2005). Community-based research partnerships: Challenges and opportunities. Journal of Urban Health, 82(2), 3-12.

Mollick, E. (2014). The dynamics of crowdfunding: An exploratory study. Journal of Business Venturing, 29(1), 1-16.

Morgan, D. L., Ataie, J., Carder, P., \& Hoffman, K. (2013). Introducing dyadic interviews as a method for collecting qualitative data. Qualitative Health Research, 23(9), 1276-1284.

Morin, S. F. (1977). Heterosexual bias in psychological research on lesbianism and male homosexuality. American Psychologist, 32, 629-637.

Morris, J. F., \& Balsam, K. F. (2003). Lesbian and bisexual women's experiences of victimization: Mental health, revictimization, and sexual identity development. Journal of Lesbian Studies, 7(4), 67-85.

Mulick, P. S., \& Wright, L. W. (2002). Examining the existence of biphobia in the heterosexual and homosexual populations. Journal of Bisexuality, 24, 45-64.

Mullins, G., \& Kiley, M. (2002). "It's a PhD, not a Nobel prize": How experienced examiners assess research theses. Studies in Higher Education, 27(4), 369-386.

Murad, M. H., Elamin, M. B., Garcia, M. Z., Mullan, R. J., Murad, A., Erwin, P. J., \& Montori, V. M. (2010). Hormonal therapy and sex reassignment: A systematic review and meta-analysis of quality of life and psychosocial outcomes. Clinical Endocrinology, 72(2), 214-231.

Murphy, J. (2016, November 4). Toronto professor Jordan Peterson takes on genderneutral pronouns. BBC News. Retrieved from https://www.bbc.com/news/worldus-canada-37875695

Murphy, S. (2018, November 27). Pride and police: The history, issues and decisions behind the debate. RNZ News. Retrieved from 
https://www.rnz.co.nz/news/national/376950/pride-and-police-the-history-issuesand-decisions-behind-the-debate

Mustanski, B. S., Garofalo, R., \& Emerson, E. M. (2010). Mental health disorders, psychological distress, and suicidality in a diverse sample of lesbian, gay, bisexual, and transgender youths. American Journal of Public Health, 100(12), 2426-2432.

Nadal, K. L. (2008). Preventing racial, ethnic, gender, sexual minority, disability, and religious microaggressions: Recommendations for promoting positive mental health. Prevention in Counseling Psychology: Theory, Research, Practice and Training, 2(1), 22-27.

Nadal, K. L., Issa, M. A., Leon, J., Meterko, V., Wideman, M., \& Wong, Y. (2011). Sexual orientation microaggressions: "Death by a thousand cuts" for lesbian, gay, and bisexual youth. Journal of LGBT Youth, 8(3), 234-259.

Neville, S., \& Henrickson, M. (2006). Perceptions of lesbian, gay and bisexual people of primary healthcare services. Journal of Advanced Nursing, 55(4), 407-415.

New Zealand Association of Psychotherapists. (2016). Mental health funding cuts: Double blow for Canterbury. Retrieved from http://www.scoop.co.nz/stories/PO1602/S00192/mental-health-funding-cutsdouble-blow-for-canterbury.htm

New Zealand Family Violence Clearinghouse. (2017). Data summaries 2017: Snapshot. Auckland: Retrieved from https://nzfvc.org.nz/sites/nzfvc.org.nz/files/Datasummaries-snapshot-2017.pdf

New Zealand Labour Party, Green Party of Aotearoa New Zealand, \& Grey Power. (2017). Inquiry into Aged Care. Wellington: Retrieved from https://www.greens.org.nz/sites/default/files/policy-pdfs/Aged Care Report Sep17.pdf

New Zealand Parliament Justice Committee. (2019). Petitions of Max Tweedie and Amanda Ashley: Ban Gay Conversion Therapy. Wellington: Retrieved from https://www.parliament.nz/resource/enNZ/SCR_92751/c6e6e71725e1e313965f8cf736f3813fd9bf9f06 
New Zealand Psychologists Board. (2002). Code of ethics for psychologists working in Aotearoa/New Zealand. Wellington: New Zealand Psychologists Board.

New Zealand Psychologists Board. (2011). Cultural competencies for psychologists registered under the Health Practitioners Competence Assurance Act (2003) and those seeking to become registered. Wellington: Retrieved from http://www.psychologistsboard.org.nz/cms_show_download.php?id=62

New Zealand Transport Agency. (2019). Updating your licence. Retrieved from https://nzta.govt.nz/driver-licences/renewing-replacing-and-updating/updatingyour-licence/

Nystrom, N. M. (1997). Oppression by mental health providers: A report by gay men and lesbians about their treatment (Doctoral thesis, University of Washington). Retrieved from http://proquest.umi.com/pqdweb?did=740113061\&Fmt=7\&clientId=63512\&RQT $=309 \&$ VName $=$ PQD

Ochs, R. (1996). Biphobia: It goes more than two ways. In B. A. Firestein (Ed.), Bisexuality: The psychology and politics of an invisible minority (pp. 240-259). Newbury Park, CA: Sage.

O’Hagan, M., Reynolds, P., \& Smith, C. (2012). Recovery in New Zealand: An evolving concept? International Review of Psychiatry, 24(1), 56-63.

Oliphant, J., Veale, J., Macdonald, J., Carroll, R., Johnson, R., Harte, M., ... Bullock, J. (2018). Guidelines for gender affirming healthcare for gender diverse and transgender children, young people and adults in Aotearoa, New Zealand. Hamilton: Transgender Health Research Lab, University of Waikato.

Olson, J., Schrager, S. M., Belzer, M., Simons, L. K., \& Clark, L. F. (2015). Baseline physiologic and psychosocial characteristics of transgender youth seeking care for gender dysphoria. Journal of Adolescent Health, 57(4), 374-380.

Orchard, S. (2017). Queer and trans 101: A super simple comic guide. Auckland: RainbowYOUTH.

O’Toole, C. J., \& Brown, A. A. (2003). No reflection in the mirror. Journal of Lesbian 
Studies, 7(1), 35-49.

Out Loud Aotearoa. (2018). Sharing the stories and wishes of queer, gender diverse, intersex, takatāpui, MVPFAFF and rainbow communities around Aotearoa's mental health and addiction services. Auckland: RainbowYOUTH.

Owen-Pugh, V., \& Baines, L. (2014). Exploring the clinical experiences of novice counsellors working with LGBT clients: Implications for training. Counselling and Psychotherapy Research, 14(1), 19-28.

Page, E. (2004). Mental health services experiences of bisexual women and bisexual men. Journal of Bisexuality, 4(1-2), 83-97.

Pallotta-Chiarolli, M., \& Martin, E. (2009). "Which sexuality? Which service?”: Bisexual young people's experiences with youth, queer and mental health services in Australia. Journal of LGBT Youth, 6(2-3), 199-222.

Patton, M. (1990). Qualitative evaluation and research methods (Second Edition). Newbury Park: Sage.

Perry, C., Thurston, M., \& Green, K. (2004). Involvement and detachment in researching sexuality: Reflections on the process of semistructured interviewing. Qualitative Health Research, 14(1), 135-148.

Pettit, M., \& Hegarty, P. (2014). Psychology and sexuality in historical time. In D. L. Tolman \& L. M. Diamond (Eds.), APA handbook of sexuality and psychology, Vol. 1: Person-based approaches (pp. 63-78). Washington, DC: American Psychological Association.

Pillow, W. S. (2003). Confession, catharsis, or cure? Rethinking the uses of reflexivity as methodological power in qualitative research. International Journal of Qualitative Studies in Education, 16(2), 175-196.

Pitts, M. K., Couch, M., Mulcare, H., Croy, S., \& Mitchell, A. (2009). Transgender people in Australia and New Zealand: Health, well-being and access to health services. Feminism \& Psychology, 19(4), 475-495.

Pitts, M. K., Smith, A., Mitchell, A., \& Patel, S. (2006). Private Lives: A report on the 
health and wellbeing of GLBTI Australians. Melbourne: Australian Research Centre in Sex, Health and Society, La Trobe University.

Pixton, S. (2003). Experiencing gay affirmative therapy: An exploration of clients' views of what is helpful. Counseling and Psychotherapy Research, 3(3), 211-215.

Platzer, H. K. (2006). Positioning identities. New York: Routledge.

Polster, H. (2003). Gender identity as a new prohibited ground of discrimination. New Zealand Journal of Public and International Law, 1(1), 157-196.

Porter, M. E., Stern, S., \& Green, M. (2017). Social Progress Index 2017. Washington, DC: Social Progress Imperative.

Poteat, T., German, D., \& Kerrigan, D. (2013). Managing uncertainty: A grounded theory of stigma in transgender health care encounters. Social Science and Medicine, 84, 22-29.

Potter, J., \& Wetherell, M. (1987). Discourse and social psychology: Beyond attitudes and behaviour. London: Sage.

PrideNZ. (2011). Phylesha Brown-Acton delivers a keynote presentation. Retrieved from http://www.pridenz.co/apog_phylesha_brown_acton_\%0Akeynote.html

Puckett, J. A., \& Levitt, H. M. (2015). Internalized stigma within sexual and gender minorities: Change strategies and clinical implications. Journal of LGBT Issues in Counseling, 9(4), 329-349.

Rachlin, K. (2002). Transgender individuals' experiences of psychotherapy. International Journal of Transgenderism, 6(1).

Radó, S. (1940). A critical examination of the concept of bisexuality. Psychosomatic Medicine, 2, 459-467.

Reading, R., \& Rubin, L. R. (2011). Advocacy and empowerment: Group therapy for LGBT asylum seekers. European Physical Education Review, 17(2), 86-98.

Reis, E. (2019). Did bioethics matter? A history of autonomy, consent, and intersex genital surgery. Medical Law Review, O(0), 1-17. 
Reisner, S. L., White, J. M., Bradford, J. B., \& Mimiaga, M. J. (2014). Transgender health disparities: Comparing full cohort and nested matched-pair study designs in a community health center. LGBT Health, 1(3), 177-184.

Relationships (Statutory References) Act, No. 3. (2005). Retrieved from http://www.legislation.govt.nz/act/public/2005/0003/latest/whole.html

Richters, J. (1997, October). Bisexuals. Paper presented at the National Lesbian, Gay, Transgender and Bisexual Health Conference, Sydney.

Riggs, D. W., Pearce, R., Pfeffer, C. A., Hines, S., White, F., \& Ruspini, E. (2019). Transnormativity in the psy disciplines: Constructing pathology in the Diagnostic and Statistical Manual of Mental Disorders and Standards of Care. The American Psychologist, 74(8), 912-924.

Riggs, D. W., \& Treharne, G. J. (2017). Decompensation: A novel approach to accounting for stress arising from the effects of ideology and social norms. Journal of Homosexuality, 64(5), 592-605.

Rishworth, P. (2007). Changing times, changing minds, changing laws - sexual orientation and New Zealand law, 1960 to 2005. International Journal of Human Rights, 11(1-2), 85-107.

Robertson, A. E. (1998). The mental health experiences of gay men: A research study exploring gay men's health needs. Journal of Psychiatric and Mental Health Nursing, 5(1), 33-40.

Robson, S. (2017, April 19). Mental health "grey zone" stopping people in need. $R N Z$ News. Retrieved from https://www.rnz.co.nz/news/national/329099/mental-health'grey-zone'-stopping-people-in-need

Ross, K. A. E., Law, M. P., \& Bell, A. (2016). Exploring healthcare experiences of transgender individuals. Transgender Health, 1(1), 238-249.

Rowe, W. (2014). Auditioning for care: Transsexual men in Ontario accessing health care. In D. Irving \& R. Raj (Eds.), Trans activism in Canada: A reader (pp. 209224). Toronto: Canadian Scholars' Press. 
Royal, T. (2012). Politics and knowledge: Kaupapa Māori and mātauranga Māori. New Zealand Journal of Educational Studies, 47(2), 30-37.

Russell, S. T., Ryan, C., Toomey, R. B., Diaz, R. M., \& Sanchez, J. (2010). lesbian, gay, bisexual, and transgender adolescent school victimization: Implications for young adult health and adjustment. Journal of School Health, 81(5), 223-230.

Ryden, J., \& Loewenthal, D. (2001). Psychotherapy for lesbians: The influence of therapist sexuality. Counselling and Psychotherapy Research, 1(1), 42-52.

Safer, J. D., Coleman, E., Feldman, J., Garofalo, R., Hembree, W., Radix, A., \& Sevelius, J. (2016). Barriers to healthcare for transgender individuals. Current Opinion in Endocrinology, Diabetes and Obesity, 23(2), 168-171.

Sandfort, T. G., de Graaf, R., Bijl, R. V, \& Schnabel, P. (2001). Same-sex sexual behavior and psychiatric disorders: Findings from the Netherlands Mental Health Survey and Incidence Study (NEMESIS). Archives of General Psychiatry, 58(1), 85-91.

Sareen, J., Jagdeo, A., Cox, B. J., Clara, I., ten Have, M., Belik, S., ... Stein, M. B. (2007). Perceived barriers to mental health service utilization in the United States, Ontario, and the Netherlands. Psychiatric Services, 58(3), 357-364.

Saulnier, C. F. (2002). Deciding who to see: Lesbians discuss their preferences in health and mental health care providers. Social Work, 47(4), 355-365.

Saulnier, C. F., \& Wheeler, E. (2000). Social action research: Influencing providers and recipients of health and mental health care for Lesbians. Affilia, 15(3), 409-433.

Scherrer, K. (2013). Culturally competent practice with bisexual individuals. Clinical Social Work Journal, 41(3), 238-248.

Schilt, K., \& Westbrook, L. (2009). Doing gender, doing heteronormativity: “Gender normals", transgender people, and the social maintenance of heterosexuality. Gender \& Society, 23(4), 440-464.

Schmidt, J. (2015). Gender diversity - Te Ara Encyclopedia of New Zealand. Retrieved from https://teara.govt.nz/en/gender-diversity/page-6\#1 
Schuetz, A. (1944). The stranger: An essay in social psychology. American Journal of Sociology, 49(6), 499-507.

Schulz, S. L. (2018). The informed consent model of transgender care: An alternative to the diagnosis of gender dysphoria. Journal of Humanistic Psychology, 58(1), 72 92.

Schützmann, K., Brinkmann, L., Schacht, M., \& Richter-Appelt, H. (2009).

Psychological distress, self-harming behavior, and suicidal tendencies in adults with disorders of sex development. Archives of Sexual Behavior, 38(1), 16-33.

Sedgwick, E. K. (1993). Tendencies. Durham: Duke University Press.

Semp, D. (2006). A public silence: Discursive practices surrounding homosexuality in public mental health services in Aotearoa/New Zealand (Doctoral thesis, University of Auckland). Retrieved from https://researchspace.auckland.ac.nz/bitstream/handle/2292/276/02whole.pdf?sequ ence $=11$

Serano, J. (2007). Whipping girl. Emeryville, CA: Seal Press.

Serano, J. (2016). No desistance, detransition and disinformation: A guide for understanding transgender children debates. Retrieved from https://medium.com/@juliaserano/detransition-desistance-and-disinformation-aguide-for-understanding-transgender-children-993b7342946e

Shaw, E., Butler, C. A., Langdridge, D., Gibson, S., Barker, M., Lenihan, P., ... Richards, C. (2012). Guidelines and literature review for psychologists working therapeutically with sexual and gender minority clients. London: British Psychological Society.

Shelton, K., \& Delgado-Romero, E. A. (2011). Sexual orientation microaggressions: The experience of lesbian, gay, bisexual, and queer clients in psychotherapy. Journal of Counseling Psychology, 58(2), 210-221.

Shipherd, J. C., Green, K. E., \& Abramovitz, S. (2010). Transgender clients: Identifying and minimizing barriers to mental health treatment. Journal of Gay \& Lesbian Mental Health, 14(2), 94-108. 
Simeonov, D., Steele, L. S., Anderson, S., \& Ross, L. E. (2015). Perceived satisfaction with mental health services in the lesbian, gay, bisexual, transgender, and transsexual communities in Ontario, Canada: An internet-based survey. Canadian Journal of Community Mental Health, 34(1), 31-44.

Singal, J. (2018). When children say they're trans. The Atlantic. Retrieved from https://www.theatlantic.com/magazine/archive/2018/07/when-a-child-says-shestrans/561749/

Skegg, K., Nada-Raja, S., Dickson, N., Paul, C., \& Williams, S. (2003). Sexual orientation and self-harm in men and women. American Journal of Psychiatry, 160(3), 541-546.

Small, Z. (2019, October 31). Trans woman awaiting surgery appeals for cash boost to ease "backlog" of almost 200. Newshub. Retrieved from https://www.newshub.co.nz/home/politics/2019/10/trans-woman-awaiting-surgeryappeals-for-cash-boost-to-ease-backlog-of-almost-200.html

Smith, L. T. (1999). Decolonizing methodologies: Research and indigenous peoples. Dunedin: University of Otago Press.

Smith, L. T. (2015). Kaupapa Māori research - some Kaupapa Māori principles. In L. Pihama, S. Tiakiwai, \& K. Southey (Eds.), Kaupapa Rangahau: A Reader (Second Edition, pp. 47-53). Hamilton: Te Kotahi Research Institute.

Social Security Act, No. 7. (1938). Retrieved from http://www.nzlii.org/nz/legis/hist_act/ssa19382gv1938n7266/

Solway, E., Estes, C. L., Goldberg, S., \& Berry, J. (2010). Access barriers to mental health services for older adults from diverse populations: Perspectives of leaders in mental health and aging. Journal of Aging and Social Policy, 22(4), 360-378.

Sparkes, A. C. (1994). Life histories and the issue of voice: Reflections on an emerging relationship. International Journal of Qualitative Studies in Education, 7(2), 165183.

Speak Up For Women. (2019). Massey University, Speak Up For Women and Feminism 2020. Retrieved from https://speakupforwomen.nz/massey-university- 
speak-up-for-women-and-feminism-2020/

Sperber, J., Landers, S., \& Lawrence, S. (2008). The health and social services needs of transgender people in Philadelphia. International Journal of Transgenderism, $8(2$ 3), 31-47.

Stop Trans Pathologization. (2012). Manifesto: International network for trans depathologization. Retrieved from https://www.stp2012.info/old/en/manifesto

Sue, D. W. (2010). Microaggressions in everyday life: Race, gender, and sexual orientation. Wiley. Hoboken, NJ: John Wiley \& Sons.

Sue, D. W., \& Torino, G. (2005). Racial-cultural competence: Awareness, knowledge, and skills. In R. T. Carter (Ed.), Handbook of racial-cultural psychology and counseling, volume 2: Training and practice (pp. 3-18). Hoboken, NJ: Wiley.

Sullivan, T. B. (2007). Evidence-based practices in mental health: Debate and dialogue on the fundamental questions. Psychiatric Services, 58(6), 882-883.

Tang, D. T. S. (2007). The research pendulum: Multiple roles and responsibilities as a researcher. Journal of Lesbian Studies, 10(3-4), 11-27.

Taylor, E. T. (2013). Transmen's health care experiences: Ethical social work practice beyond the binary. Journal of Gay \& Lesbian Social Services, 25(1), 102-120.

Temple Newhook, J., Pyne, J., Winters, K., Feder, S., Holmes, C., Tosh, J., ... Pickett, S. (2018). A critical commentary on follow-up studies and "desistance" theories about transgender and gender-nonconforming children. International Journal of Transgenderism, 19(2), 212-224.

Tennyson, R. D., \& Volk, A. (2015). Learning theories and educational paradigms. In J. D. Wright (Ed.), International Encyclopedia of the Social \& Behavioral Sciences (Second Edition, pp. 699-711). Amsterdam: Elsevier.

Tolan, J., \& Cameron, R. (2003). Skills in person-centred counselling \& psychotherapy. London: Sage.

Toomey, R. B., Ryan, C., Diaz, R. M., Card, N. A., \& Russell, S. T. (2010). Gendernonconforming lesbian, gay, bisexual, and transgender youth: School victimization 
and young adult psychosocial adjustment. Developmental Psychology, 46(6), 15801589.

Treharne, G. J. (2011). Questioning sex/gender and sexuality: Reflections on recruitment and stratification. Gay \& Lesbian and Psychology Review, 7(2), 132154.

Tryon, W. W. (2010). Competencies in adult clinical psychology. In J. C. Thomas \& M. Hersen (Eds.), Handbook of clinical psychology competencies (pp. 1-42). New York, NY: Springer.

UNICEF. (2017). Building the future: Children and the sustainable development goals in rich countries. Florence: UNICEF Office of Research - Innocenti.

Ussher, J. M. (2010). Are we medicalizing women's misery? A critical review of women's higher rates of reported depression. Feminism \& Psychology, 20(1), 935.

Vance S. R. Jr., Cohen-Kettenis, P. T., Drescher, J., Meyer-Bahlburg, H. F., Pfäfflin, F., \& Zucker, K. J. (2010). Opinions about the DSM gender identity disorder diagnosis: Results from an international survey administered to organizations concerned with the welfare of transgender people. International Journal of Transgenderism, 12(1), 1-14.

Vaughan, G., \& Hansen, C. (2004). 'Like Minds, Like Mine': A New Zealand project to counter the stigma and discrimination associated with mental illness. Australasian Psychiatry, 12(2), 113-117.

Veale, J., Byrne, J., Tan, K., Guy, S., Yee, A., Nopera, T., \& Bentham, R. (2019). Counting ourselves: The health and wellbeing of trans and non-binary people in Aotearoa New Zealand. Hamilton: Transgender Health Research Lab, University of Waikato.

Veale, J., Peter, T., Travers, R., \& Saewyc, E. M. (2017). Enacted stigma, mental health, and protective factors among transgender youth in Canada. Transgender Health, 2(1), 207-216.

Victoria University Research Policy Group. (2018). Human Ethics Guidelines. 
Wellington: Retrieved from https://www.wgtn.ac.nz/documents/policy/researchpolicy/appendix-a-human-ethics-committee-guidelines.pdf

Villenas, S. (1996). The colonizer/colonized Chicana ethnographer: Identity, marginalization, and co-optation in the field. Harvard Educational Review, 66(4), 711-731.

Violeta, K. J., \& Langer, S. J. (2017). Integration of desire, sexual orientation, and female embodiment of a transgender woman previously diagnosed with autism spectrum disorder: A case report. Journal of Gay \& Lesbian Mental Health, 21(4), 1935-9705.

Vipond, E. (2015). Resisting transnormativity: Challenging the medicalization and regulation of trans bodies. Theory in Action, 8(2), 21-44.

Wagle, T., \& Cantaffa, D. T. (2008). Working our hyphens. Qualitative Inquiry, 14(1), $135-159$.

Waitangi Tribunal. (2019). About the Waitangi Tribunal. Retrieved from https://waitangitribunal.govt.nz/about-waitangi-tribunal/

Wallerstein, N. B., \& Duran, B. (2006). Using community-based participatory research to address health disparities. Health Promotion Practice, 7(3), 312-323.

Walters, L. (2019, May 30). Mental health gets long-overdue cash injection. Newsroom. Retrieved from https://www.newsroom.co.nz/2019/05/30/612432/a-focus-onfrontline-mental-health

Webb-Liddall. (2019, February 11). Government should spend money on cancer treatment, not gender reassignment - Mark Richardson. Newshub. Retrieved from https://www.newshub.co.nz/home/new-zealand/2019/02/government-shouldspend-money-on-cancer-treatment-not-gender-reassignment-mark-richardson.html

Welch, S., Collings, S. C. D., \& Howden-Chapman, P. (2000). Lesbians in New Zealand: Their mental health and satisfaction with mental health services. Australian and New Zealand Journal of Psychiatry, 34(2), 256-263.

White, B. P., \& Fontenot, H. B. (2019). Transgender and non-conforming persons' 
mental healthcare experiences: An integrative review. Archives of Psychiatric Nursing, 33(2), 203-210.

Whitehead, J., Shaver, J., \& Stephenson, R. (2016). Outness, stigma, and primary health care utilization among rural LGBT Populations. PLoS ONE, 11(1), e0146139.

White Hughto, J. M., \& Reisner, S. L. (2016). A systematic review of the effects of hormone therapy on psychological functioning and quality of life in transgender individuals. Transgender Health, 1(1), 21-31.

White Hughto, J. M., Reisner, S. L., \& Pachankis, J. E. (2015, December 1). Transgender stigma and health: A critical review of stigma determinants, mechanisms, and interventions. Social Science and Medicine, 147, 222-231.

Wiepjes, C. M., Nota, N. M., de Blok, C. J. M., Klaver, M., de Vries, A. L. C., Wensing-Kruger, S. A., ... den Heijer, M. (2018). The Amsterdam Cohort of Gender Dysphoria Study (1972-2015): Trends in prevalence, treatment, and regrets. Journal of Sexual Medicine, 15(4), 582-590.

Wiggins, W. (2017, May 26). Budget 2017: Mental health funding "distressing." The New Zealand Herald. Retrieved from http://www.nzherald.co.nz/nz/news/article.cfm?c_id=1\&objectid=11863285

Wi-Hongi, A., Greig, A., \& Hazenburg, E. (2017). Towards a model of informed consent: trans healthcare in Aotearoa New Zealand. In E. Hazenberg \& M. Meyerhoff (Eds.), Representing trans: Linguistic, legal and everyday perspectives (pp. 153-170). Wellington: Victoria University Press.

Willging, C. E., Salvador, M., \& Kano, M. (2006). Unequal treatment: Mental health care for sexual and gender minority groups in a rural state. Psychiatric Services, $57(6), 867-870$.

Williams, H. W. (1871). A dictionary of the Mãori language (Third Edition). Wellington: Government Print.

Wilson, M. (2013). Impact of women's political leadership on democracy and development in New Zealand. In editors4change (Ed.), The impact of women's political leadership on democracy and development: Case studies from the 
Commonwealth (pp. 36-63). London: Commonwealth Secretariat.

Wisneski, D. B. (2013). bell hooks: Scholar, cultural critic, feminist, and teacher. In J. D. Kirylo (Ed.), A Critical Pedagogy of Resistance: 34 Pedagogues We Need to Know (pp. 73-76). Rotterdam: Sense Publishers.

Woody, G. E., VanEtten-Lee, M. L., McKirnan, D., Donnell, D., Metzger, D., Seage, G., ... HIVNET VPS 001 Protocol Team. (2001). Substance use among men who have sex with men: comparison with a national household survey. Journal of Acquired Immune Deficiency Syndromes, 27(1), 86-90.

Wright, T. (2016, October 31). What's behind New Zealand's mental health funding crisis? Newshub. Retrieved from http://www.newshub.co.nz/home/health/2016/11/whats-behind-new-zealandsmental-health-funding-crisis.html

Wynn, R., \& West-Olatunji, C. (2009). Use of culture-centered counseling theory with ethnically diverse LGBT clients. Journal of LGBT Issues in Counseling, 3(3-4), $198-214$.

Yarns, B. C., Abrams, J. M., Meeks, T. W., \& Sewell, D. D. (2016). The mental health of older LGBT adults. Current Psychiatry Reports, 18, 1-11.

Yule, M. A., Brotto, L. A., \& Gorzalka, B. B. (2013). Mental health and interpersonal functioning in self-identified asexual men and women. Psychology and Sexuality, $4(2), 136-151$. 